\title{
Colloidal Crystals of Spheres and Cubes in Real and Reciprocal Space
}

Janne-Mieke Meijer 


\section{Universiteit Utrecht}

\section{Cover:}

Front: TXM image of the fluid-crystal interface of colloidal hematite cubes coated with a silica layer that are settling in ethanol under the influence of an external magnetic field.

Back: 2D SAXS pattern of the same sample taken in the crystalline part with a sample rotation of $45^{\circ}$ that shows high order Bragg peaks.

Printed by: Gildeprint. 


\title{
Colloidal Crystals of Spheres and Cubes in Real and Reciprocal Space
}

\section{Colloïdale Kristallen van Bollen en Kubussen in de Reële en Reciproke Ruimte}

(met een samenvatting in het Nederlands)

\author{
Proefschrift
}

\begin{abstract}
ter verkrijging van de graad van doctor aan de Universiteit Utrecht op gezag van de rector magnificus, prof. dr. G.J. van der Zwaan, ingevolge het besluit van het college voor promoties in het openbaar te verdedigen op maandag 12 januari 2015 des middags te 2.30 uur
\end{abstract}

door

\section{Janne-Mieke Meijer}

geboren op 21 april 1985 te Utrecht 
Promotoren:

Copromotor:
Prof. dr. H.N.W. Lekkerkerker

Prof. dr. A.P. Philipse

Dr. A.V. Petukhov

Dit proefschrift werd (mede) mogelijk gemaakt met financiële steun van de Koninklijke Nederlandse Akademie van Wetenschappen door middel van het Akademiehoogleraarschap van H.N.W. Lekkerkerker. 


\section{CONTENTS}

Publication list

Chapter I - General Introduction

I.I. Self-organization in colloidal systems

1.2. Ordered structures of colloidal spheres

1.3. Ordered structures of colloidal cubes

I.4. Colloidal crystal preparation

1.5. Characterization of colloidal crystals

I.6. Outline of this thesis

Chapter 2 - Preparation and Characterization of Colloidal Crystals for Synchrotron and Free Electron Laser X-ray Studies 23

2.I. Introduction 24

2.2. Experimental $\quad 24$

2.3. Results 26

2.4. Conclusions \& Outlook $\quad 32$

2.5. X-ray diffraction experiments of colloidal crystals of spheres 33

Chapter 3 - Double Hexagonal Close Packed Structure Revealed in a Single Colloidal Crystal Grain by Bragg Rod Analysis 37

3.I. Introduction 38

3.2. Experimental 38

3.3. Bragg rod analysis $\quad 39$

3.4. 3D reconstruction $\quad 46$

3.5. Conclusions 46

Chapter 4 - Inducing Defects in Colloidal Crystals with Thermosensitive PNIPAM PARTICLES 51

4.I. Introduction $\quad 52$

4.2. Experimental $\quad 54$

4.3. Results \& Discussion $\quad 57$

4.4 Conclusion $\quad 64$

4.5. Outlook 64

$\begin{array}{ll}\text { Part B - Cubes } & 67\end{array}$

Chapter 5 - Preparation and Characterization of Colloidal Cubes 69

$\begin{array}{ll}\text { 5.I. Introduction } & 70\end{array}$

5.2. Experimental 71

$\begin{array}{ll}\text { 5.3. Colloidal cube properties } & 74\end{array}$

5.4. Conclusions 81 
Chapter 6 - Self-assembly of Colloidal Cubes via Vertical Deposition 85

6.I. Introduction $\quad 86$

$\begin{array}{lr}\text { 6.2. Experimental } & 86\end{array}$

6.3. Results $\quad 88$

\begin{tabular}{ll} 
6.4. Discussion & 93 \\
\hline 6.5. Concusions & 98
\end{tabular}

6.5. Conclusions 95

Chapter 7 - Experimental Evidence of Optimal Packings in Convectively Assembled Crystals of Colloidal Cubes 99

7.I. Introduction 100

7.2. Experimental 101

$\begin{array}{ll}\text { 7.3. Results \& Discussion } & 106\end{array}$

$\begin{array}{ll}\text { 7.4. Conclusion } & 114\end{array}$

$\begin{array}{ll}\text { 7.5. Outlook } & 115\end{array}$

Chapter 8 - Self-Assembly of Colloidal Cubes Induced by Sedimentation 19

$\begin{array}{ll}\text { 8.I. Introduction } & 120\end{array}$

$\begin{array}{ll}\text { 8.2. Experimental } & 120\end{array}$

$\begin{array}{ll}\text { 8.3. Results and Discussion } & 122\end{array}$

$\begin{array}{ll}\text { 8.4. Conclusions } & 130\end{array}$

8.5. Outlook |31

Chapter 9 -Sedimentary Crystals of Magnetic Hematite Cubes and

the Influence of an External Magnetic Field

9.I. Introduction 134

$\begin{array}{ll}\text { 9.2. Experimental } & 134\end{array}$

$\begin{array}{ll}\text { 9.3. Results and Discussion } & 137\end{array}$

$\begin{array}{ll}\text { 9.4. Conclusions } & 146\end{array}$

\begin{tabular}{l|l} 
SUMMARY & 152
\end{tabular}

$\begin{array}{ll}\text { Nederlandse SamenVatting voOR Iedereen } & 155\end{array}$

$\begin{array}{ll}\text { ACKNOWLEDGEMENTS / DANKWOORD } & \text { I59 }\end{array}$

$\begin{array}{lll}\text { Curriculum Vitae } & 163\end{array}$ 


\section{Publication List}

\section{This thesis is partly based on the following publications:}

- D.V. Byelov, J.M. Meijer, I. Snigireva, A. Snigirev, L. Rossi, E. van den Pol, A. Kuijk, A. Philipse, A. Imhof, A. van Blaaderen, G.J. Vroege and A.V. Petukhov, In situ hard X-ray microscopy of self-assembly in colloidal suspensions, RSC Advances, 2013, 3, 15670 15677 (Chapter 1)

- R. Dronyak, J. Gulden, O.M. Yefanov, A. Singer, T. Gorniak, T. Senkbeil, J.M. Meijer, A. Al-Shemmary, J. Hallmann, D.D. Mai, T. Reusch, D. Dzhigaev, R.P. Kurta, U. Lorenz, A.V. Petukhov, S. Düsterer, R. Treusch, M.N. Strikhanov, E. Weckert, A.P. Mancuso, T. Salditt, A. Rosenhahn, and I.A. Vartanyants, Dynamics of colloidal crystals studied by pump-probe experiments at FLASH, Phys. Rev. B, 2012, 86, 064303 (Chapter 2)

- V. Zozulya, J.M. Meijer, A. Shabalin, A. Ricci, F. Westermeier, R.P. Kurta, U. Lorenz, A. Singer, O. Yefanov, A.V. Petukhov, M. Sprung and I.A. Vartanyants, In situ X-ray crystallographic study of the structural evolution of colloidal crystals upon heating, J. Appl. Cryst., 2013, 46, 903-907 (Chapter 2)

- J.M. Meijer, A. Shabalin, R. Dronyak, O.M. Yefanov, A. Singer, R.P. Kurta, U. Lorenz, O. Gorobstov, D. Dzhigaev, J. Gulden, D.V. Byelov, A.V. Zozulya, M. Sprung, I.A. Vartanyants and A.V. Petukhov, Double hexagonal close packed structure revealed in a single colloidal crystal grain by Bragg rod analysis, J. Appl. Cryst, 2014, 47, 1199-1204 (Chapter 3)

- J.M. Meijer, F. Hagemans, L. Rossi, D.V. Byelov, S.I.R. Castillo, A. Snigirev, I. Snigireva, A.P. Philipse, and A.V. Petukhov, Self-Assembly of Colloidal Cubes via Vertical Deposition, Langmuir, 2012, 28, 7631-7638 (Chapter 6)

- J.M. Meijer, D.V. Byelov, L. Rossi, A. Snigirev, I. Snigireva, A.P. Philipse, and A.V. Petukhov, Self-assembly of colloidal hematite cubes: a microradian X-ray diffraction exploration of sedimentary crystals, Soft Matter, 2013, 9, 10729-10738 (Chapter 9)

\section{Manuscripts in preparation:}

- A. Shabalin, J.M. Meijer, A.V. Petukhov, I.A. Vartanyants et al. Reconstruction of a colloidal crystal grain with CDXI. (Chapter 3)

- J.M. Meijer, C. Benedetti, D. Verwijmeren, J. Hilhorst, A.V. Petukhov. Inducing defects in colloidal crystals with thermosensitive PNIPAm particles. (Chapter 4)

- J.M. Meijer, V. Meester, A.P. Philipse, A.V. Petukhov. Experimental Evidence for optimal packings in convectively assembled crystals of colloidal cubes. (Chapter 7)

- J.M. Meijer, A. Pal, H.N.W. Lekkerkerker, A.P. Philipse, A.V. Petukhov. Self-assembly of colloidal cubes induced by sedimentation. (Chapter 8) 


\section{Other publications by the author:}

- E.A. Sulyanova, A. Shabalin, A.V. Zozulya, J.M. Meijer, D. Dzhigaev, O. Gorobtsov, R.P. Kurta, S. Lazarev, U. Lorenz, A. Singer, O. Yefanov, I. Zaluzhnyy, I. Besedina, M. Sprung, A.V. Petukhov, I. A. Vartanyants, Structural evolution of colloidal crystal films in the process of melting revealed by Bragg peak analysis. (Submitted)

- $\quad$ A. Pal, J.M. Meijer, J.R. Wolters, W. Kegel and A.V. Petukhov. Structure and Stacking Order in Crystals of Asymmetric Dumbbell-like Colloids, (Submitted)

- D. Kleshchanok, J.M. Meijer, A.V. Petukhov, G. Portale, and H.N.W. Lekkerkerker, Sedimentation and depletion attraction directing glass and liquid crystal formation in aqueous platelet/sphere mixtures, Soft Matter, 2012, 8, 191-197

- D. Kleshchanok, P. Holmqvist, J.M. Meijer, H.N.W. Lekkerkerker, Lyotropic Smectic B Phase Formed in Suspensions of Charged Colloidal Platelets, J. Am. Chem. Soc., 2012, 134, 5985-5990

- A.B.G.M. Leferink op Reinink, J.M. Meijer, D. Kleshchanok, D.V. Byelov, G.J. Vroege, A.V. Petukhov, and H.N.W. Lekkerkerker, 3D structure of nematic and columnar phases of hard colloidal platelets, J. Phys-Cond. Matt., 2011, 23, 194110

- D. Kleshchanok, J.M. Meijer, A.V. Petukhov, G. Portale, and H.N.W. Lekkerkerker, Attractive glass formation in aqueous mixtures of colloidal gibbsite platelets and silica spheres, Soft Matter, 2011, 7, 2832-2840

- J.M. Meijer, L. Aarts, B.M. van der Ende, T.J.H. Vlugt, A. Meijerink, Downconversion for solar cells in $\mathrm{YF}_{3}: \mathrm{Nd}^{3+}, \mathrm{Yb}^{3+}$, Phys. Rev. B, 2010, 81, 035107.

- V.W.A. de Villeneuve, P.S. Miedema, J.M. Meijer and A.V. Petukhov, Grain size effects on lateral islands in hard-sphere crystals, Europhys. Lett., 2007, 79, 56001

- J.M. Meijer, V.W.A. de Villeneuve, A.V. Petukhov, In-plane stacking disorder in polydisperse hard sphere crystals, Langmuir, 2007, 23, $3554-3560$. 


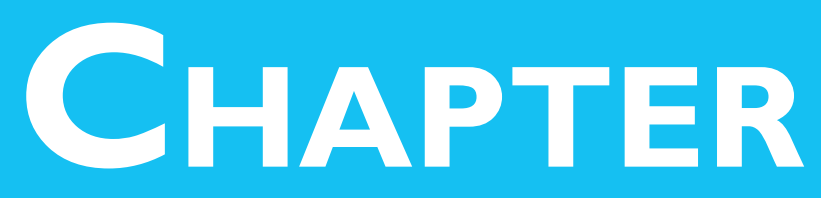

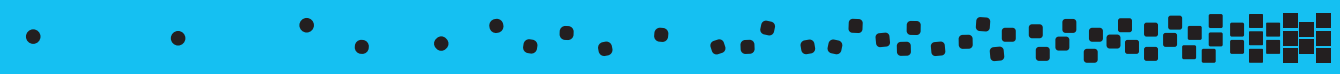
General Introduction 


\section{I.I. Self-organization in colloidal systems}

The research presented in this thesis deals with the self-organization of colloidal particles into crystalline phases. Colloidal particles, or simply colloids, are classified as particles dispersed in a continuous medium with one of their dimensions between $1 \mathrm{~nm}$ and $1 \mu \mathrm{m}^{[1]}$. Colloids are characterized by observable Brownian motion originating from the thermal energy of the particles that counteracts their sedimentation.

One of the most remarkable phenomena exhibited by concentrated suspensions of colloids is the spontaneous transition from fluid-like structures to those exhibitinglong-range spatial and/or orientational order (colloidal crystals and colloidal liquid crystals). This behavior is similar to phase transitions observed in atomic or molecular systems. The statistical mechanics of colloids is actually analogous to that of atoms, except that the bare potential has to be replaced by the potential of mean force ${ }^{[2,3]}$. For colloids the potential of mean force can be varied from steeply repulsive to deeply attractive ${ }^{[4]}$, by modifying the colloidal surface chemically, changing the solvent properties (e.g. the Debye length) or adding a depletant ${ }^{[5-7]}$. The advantage of colloids over atoms is that due to the larger size of colloids, they can be studied on a single particle level with optical microscopy. In addition, in a colloidal systems processes such as phase transitions are very much slower than in the atomic systems. All these above mentioned properties have gained colloids the label "model atoms"[8-10].

The fact that self-organization into can occur in suspensions in which the interparticle forces are purely repulsive was first explained in the 1930 s by the theoretical work of Kirkwood ${ }^{[1,12]}$ and Onsager ${ }^{[13]}$. They showed that the competition of entropy contributions - a gain of free volume entropy in the ordered state compensates a loss of configurational or orientational entropy in the fluid state - drives the ordering transition. For spheres the transition occurs in the form of a fluid state to a crystal state ${ }^{[11,12]}$, which was confirmed in the 1950 s by early computer simulations ${ }^{[14,15]}$. Subsequent computer simulations of Hoover and Ree showed for hard spheres that between the volume fractions $\phi_{\mathrm{f}}=0.494$ and $\phi_{\mathrm{s}}=0.545$ the isotropic fluid will transform in a coexisting fluid and crystal phases ${ }^{[16]}$. A daily life example of a colloidal crystal of spheres is the natural gemstone opal. The internal structure of these gemstones consists of densely packed, highly ordered arrays of small spherical silica colloids. The sphere size is comparable to the wavelength of visible light and the diffraction by the crystal causes the opal's beautiful appearance ${ }^{[17,18]}$.

Colloidal crystals can be made artificially and are of interest as model systems for studying crystal phenomena ${ }^{[19,20]}$ and as a class of new functional nanomaterials which can be fabricated with a bottom-up approach ${ }^{[21]}$. The latter is attributed to their dimensions and spontaneous self-assembly. In addition, significant progress in colloidal synthesis has yielded particles with many diverse shapes, such as plates ${ }^{[22]}$, boards ${ }^{[23,24]}$, dumbbells ${ }^{[25-27]}$, cubes $^{[28-32]}$, peanuts ${ }^{[33,34]}$ and polyhedra $^{[35-37]}$. This progress allows one to experimentally explore the effect of anisotropic colloidal shapes on the phase behavior and crystal symmetry, which has been already predicted by theory and simulations ${ }^{[38,39]}$.

In this thesis we focus on colloidal crystals composed of spheres, as described in Part A, and 
composed of cubes, as described in Part B. In Part A we aim to elucidate the properties of the structures present in colloidal sphere crystals. We are particularly interested in the structure and dynamics of both the crystals and defects, which have received limited attention ${ }^{[40-42]}$. The main aim of Part B is to study the colloidal crystals formed by micron-sized colloidal cubes. A topic which has been experimentally hardly addressed yet ${ }^{[29,43]}$. We are particularly interested in assessing the effect of the cubic shape and magnetic interactions on the outcome of the selfassembly process. To put the work of this thesis into context we briefly review previous work on colloidal sphere crystals and colloidal cube crystals in Section 1.2 and Section 1.3, respectively.

\section{I.2. Ordered structures of colloidal spheres}

Although the first colloidal crystals were already observed in the systems of charged latex particles in the 1950s and $1960 \mathrm{~s}^{[44]}$, the experimental realization of colloidal spheres that interact via a hard-sphere potential came in the 1980s in the form of sterically stabilized silica particles ${ }^{[45]}$, and later also as sterically stabilized PMMA particles ${ }^{[46]}$. These colloidal systems did indeed show the fluid-crystal transition ${ }^{[47,48]}$ and the coexisting isotropic fluid-crystal concentrations observed by Pusey and van Megen ${ }^{[47]}$ were in close agreement with the simulations of Hoover and Ree ${ }^{[16]}$. In addition Pusey and van Megen observed that above $\phi=0.58$ crystallization is inhibited and a glass phase will form. Remarkably, microgravity experiments in space have shown that without gravitational effects crystallization will occur at all volume factions and the glass phase disappears ${ }^{[49]}$, a result that could be reproduced on earth by using particles that were density matched by the solvent ${ }^{[50]}$.

Since then colloidal crystals of spheres in suspension have been used as model systems for the study of several crystallization aspects, such as crystal structure ${ }^{[19,20,51-53]}$, crystal nucleation ${ }^{[54,55]}$ and crystal melting ${ }^{[56,57]}$. In addition, the potential of colloidal crystals of spheres as functional materials, such as for photonic structures, has been investigated by fabricating dried colloidal crystals via several routes ${ }^{[58-61]}$. These studies have led to a good understanding of the crystal structure but also revealed the presence of several types of defects. These defects, analogous to those observed in atomic crystals, degrade the optical properties of the crystals and has led to detailed investigations of the defect characteristics and defect behavior in real space and time $e^{[40-42,62-66]}$.

The crystals formed by colloidal spheres consists of close-packed hexagonal layers of spheres stacked on top of each other. Each layer can occupy one of three specific positions, A, B and $\mathrm{C}$ as illustrated in Figure 1.1.a $\mathrm{a}^{[67]}$. The two simplest stacking sequences of these layers that are periodic are hexagonal close-packed (HCP) with an $\mathrm{ABAB}$... stacking and the face-centered cubic (FCC) with ABCABC... stacking and are shown in Figure 1.1.b. Due to a small free-energy difference between $\mathrm{FCC}$ and $\mathrm{HCP}^{[68]}$, experimentally a random mixture of the two stacking types is often observed, the so-called random hexagonal close-packed (RHCP) structure ${ }^{[52]}$. Figure 1.1.c shows a schematic representation of a RHCP structure where the HCP layers in between FCC layers are considered to be stacking defects. There are also other types of defects present in colloidal crystals of spheres, such as point defects, line defects, planar defect and 

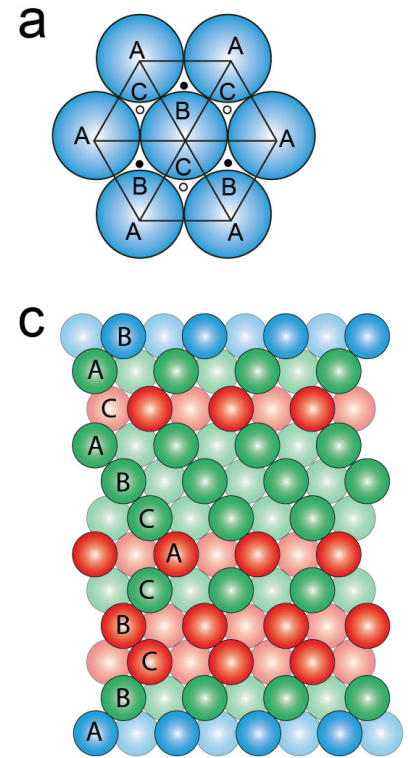

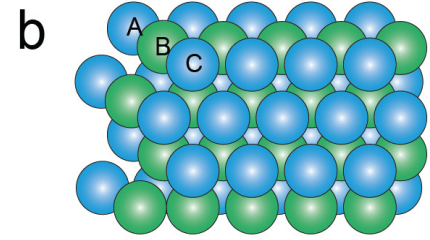

FCC

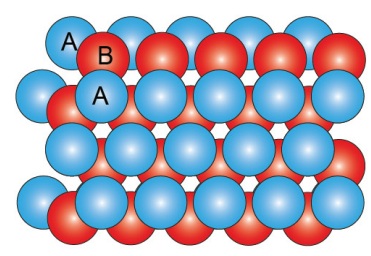

$\mathrm{HCP}$

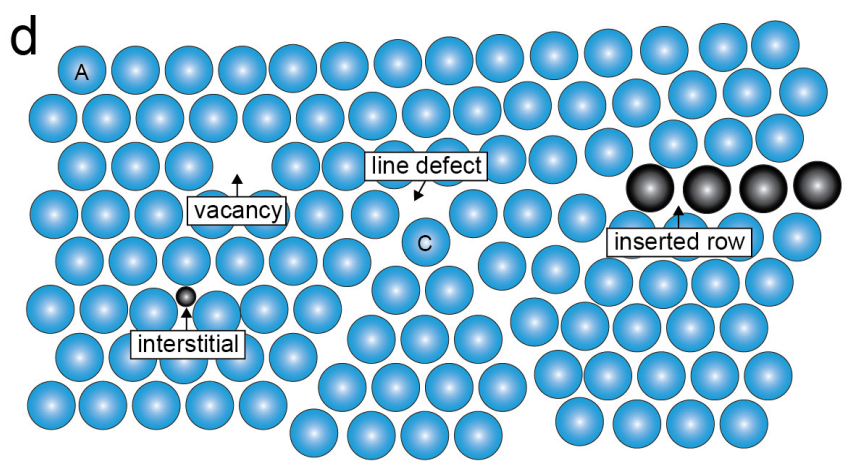

Figure I.I. a) Top view of A, B and C positions in a hexagonal close packed layers. b) Top view of stacking of 3 close-packed layers in FCC and HCP fashion. c) Side view of an example of an RHCP stacking sequence $A B C B C A C B$. The local environment changes back and forth from FCC (green) and to HCP (red). The first and last layer are not assigned FCC or HCP because a top and bottom layer are needed for assignment.

d) Several types of in-plane defects that are can occur in colloidal crystals of spheres.

bulk defects. Figure 1.1.d shows a few examples of typical defects that can occur in-plane in a hexagonal close-packed layer.

In this thesis we focus on two different types of colloidal crystals of spherical colloids: dried crystals and colloidal crystals formed in suspension. Dried crystal structures are typically used as a template for photonic applications but have been mainly investigated via microscopy techniques. The characterization of the structures and defects, as well as the effect of strong optical field, could greatly benefit from the improved X-ray diffraction methods that are available today, which allow larger sample volumes and provide better statistics. Crystals formed in suspension have the benefit that the particles still undergo Brownian motion such that the crystal structures are dynamic. One of the area's that is still relatively unexplored in these colloidal crystals is that of defect diffusion, while understanding these processes on a single particle level is of great interest for materials science.

\section{I.3. Ordered structures of colloidal cubes}

In the last 20 years improvements in colloidal synthesis have resulted in the possibility to synthesize colloids with cubic-like shapes. In fact it is even possible to produce various colloidal cubes with sharp edges ${ }^{[31,32]}$, rounded edges ${ }^{[28,29,69,70]}$ or truncated edges ${ }^{[71,72]}$. The rounded edged cubes are a special case, because these can be mathematically described by a superball shape 


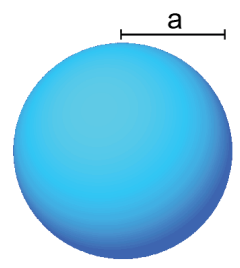

$\mathrm{m}=2$

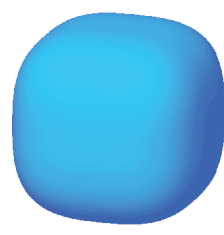

$m=3$

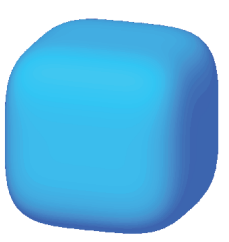

$\mathrm{m}=4$

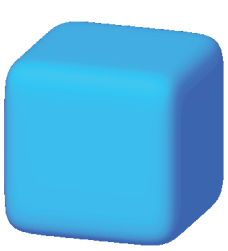

$m=8$

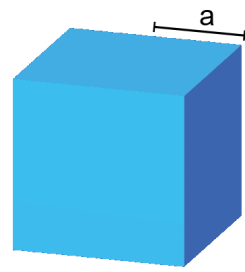

$\mathrm{m} \rightarrow \infty$

Figure I.2. Superball particles with different values of the shape parameter $m$.

defined by ${ }^{[73]}$ :

$$
\left|\frac{x}{a}\right|^{m}+\left|\frac{y}{a}\right|^{m}+\left|\frac{z}{a}\right|^{m} \leq 1
$$

where $m$ is the shape parameter, which indicates the extent of deformation from a sphere $(m=2)$ to a cube $(m \rightarrow \infty)$ and $a$ is half the edge length $D$. Figure 1.2 shows a representation of superballs with different $m$-values.

The experimental availability of superball shaped particles has inspired detailed studies of the crystalline structures and even the phase behavior of hard superballs via theory and simulation ${ }^{[73-79]}$. This was also driven by the fact that both for hard spheres, as discussed in the previous section, and hard cubes ${ }^{[80,81]}$ the phase behavior and crystal structures have already been predicted. For a perfect cube the thermodynamic stable crystal structure is a simple cubic (SC) lattice while for a sphere this is the FCC lattice. Knowing these structures for these two extreme superball shapes, the question remains what the effect of an intermediate $m$-value, or the roundness of the cube corners, will be on their close-packed structures.

Pioneering computer studies were performed by Jiao et al. who investigated the optimal packings of superballs in both two and three dimensions $(2 \mathrm{D} \text { and } 3 \mathrm{D})^{[73,74]}$. For $2 \mathrm{D}$ they showed that superdisks (the $2 \mathrm{D}$ equivalent of superballs) can be optimally packed into a hexagonal-like $\Lambda_{0}$-lattice as well as a rhombic $\Lambda_{1}$-lattice ${ }^{[74]}$. The lattice vectors of these lattices change with $m$ and the maximal packing density increases significantly for $m>2$, due to the broken rotational symmetry of the sphere. When $m>2.572$ the close-packed structure is the $\Lambda_{1}$-lattice, where as

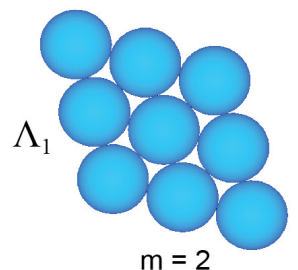

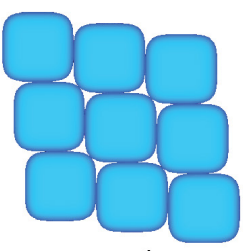

$\mathrm{m}=4$

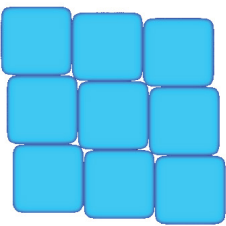

$\mathrm{m}=8$

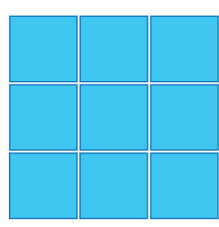

$m \rightarrow \infty$

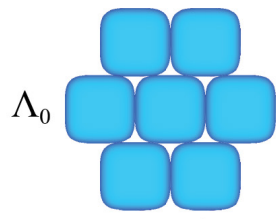

$\mathrm{m}=4$

Figure I.3. $\Lambda_{1}$-lattice for cubes with different shape parameters $m$ and $\Lambda_{0}$-lattice for $m=4$. 


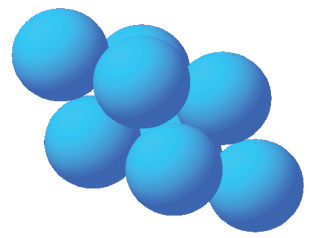

$\mathrm{m}=2$

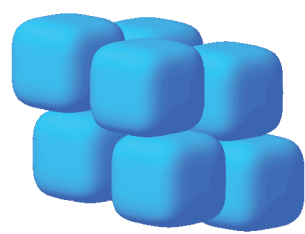

$\mathrm{m}=4$

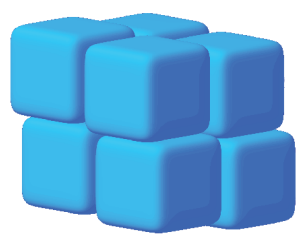

$\mathrm{m}=8$

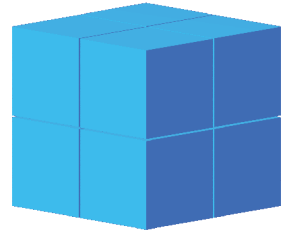

$\mathrm{m} \rightarrow \infty$

Figure I.4. $C_{1}$-lattice for rounded cubes using the superball model with different shape parameters $m$.

it is the $\Lambda_{0}$-lattice whenever $2<m<2.572$. Figure 1.3 shows a schematic representation of the $\Lambda_{1}$-lattice for different $m$, where it is clear that the increase in density is caused by the contacts between the rounded cube corners. As a result for $m=2$ to $m \rightarrow \infty$ the $\Lambda_{1}$-lattice describes the continuous transformation of a hexagonal lattice to a simple square lattice. In contrast, the $\Lambda_{0}$-lattice consists of rows of particles that are offset by $a$ and is also shown for $m=4$ in Figure 1.3. The $\Lambda_{0}$-lattice is clearly less affected by the roundness of the cube corners, which explains the lower packing density at higher $m$. For 3D Jiao et al. found two families of Bravais lattice packings, the $\mathrm{C}_{0}$-lattice and $\mathrm{C}_{1}$-lattice ${ }^{[73]}$. Analogous to the $2 \mathrm{D}$ result the $\mathrm{C}_{0}$-lattice provides the densest packing for $2<m<2.308$ while for $m>2.308$ it is the $\mathrm{C}_{1}$-lattice. The $\mathrm{C}_{1}$-lattice describes the deformation from a FCC lattice for a sphere $(m=2)$ to a SC lattice for a perfect cube $(m \rightarrow \infty)$, as illustrated in Figure 1.4. Again it is clear that contact between the rounded cube corners present in this structure is the cause of the higher packing density.

Simulations of the phase behavior of hard superball shaped particles were performed in $2 \mathrm{D}$ by Avendaño et al. ${ }^{[77] *}$ and in $3 \mathrm{D}$ by both Batten et al. ${ }^{[75]}$ and $\mathrm{Ni}$ et al. ${ }^{[76]}$. They showed that the optimal packings with the highest packing density, the $\Lambda_{1}$ in $2 \mathrm{D}$ and $\mathrm{C}_{1}$ in $3 \mathrm{D}$, are indeed realized as the thermodynamic stable phases. The lattices will deform slightly at volume fractions below close-packing due to the increase of free volume per particle. In addition, the crystal structures are preceded by liquid or plastic crystal phases, in which long-range positional order is present but orientational order is absent. In $2 \mathrm{D}$ this phase was only observed for $2<m<4.504$ and labeled a rotator hexagonal phase $\mathrm{e}^{[77]}$. In $3 \mathrm{D}$ the phase occurred for $2<m<6$ and was found to be a plastic FCC crystal. In addition, as expected in 3D for superballs with $m \rightarrow \infty$ the SC lattice was found, for which additional simulations have shown that a significant number of vacancies can be present ${ }^{[79]}$.

An interesting addition to these simulations of freely rotating cubes with rounded edges, are the simulations of cubes with fixed parallel orientations that were performed by Marechal

\footnotetext{
${ }^{\star}$ It has to be noted that the works of Avendano et al. ${ }^{[78]}$ (and Marechal et al. ${ }^{[79]}$ ) did not describe the particles with a superdisk (or superball) shape but a rounded square constructed from circles, rectangles and squares (or cube constructed from spheres, cylinders, square prisms and cubes). Therefore, the shapes are characterized by different parameters, $\zeta$ (square) and s (cube), to describe the effective roundness of the cube corners, which we have converted into a corresponding $\mathrm{m}$, using the conversion suggested by the authors, to be able to correlate their findings to the cubes used in this thesis.
} 
et al. ${ }^{[78] \star}$. They showed that for $2<m<3.08$ a first order transition from a fluid will occur into either a deformed FCC lattice with two different cube orientations or into a sheared SC lattice. In addition, they surprisingly found for $m>3.08$ a second order freezing transition, which was previously only found for oriented perfect cubes ${ }^{[80,82,83]}$.

Experimentally, the structures of superballs have been investigated as well. Noteworthy are the studies of Zhao et al.$^{[84]}$ and Zhang et al. ${ }^{[69]}$ that actually inspired two of the above mentioned phase behavior studies via computer simulations ${ }^{[76,77]}$. Zhao et al. investigated the phase behavior in $2 \mathrm{D}$ of rounded squares prepared via lithography methods and found a rotator and rhombic structure. Zhang et al. studied nanometer sized cubes in solution that were rounded by an excess of ligands and found a continuous phase transition from SC to FCC.

Recently, Rossi et al. ${ }^{[29,30]}$ prepared micron-sized hollow colloidal silica cubes that are a realization of a colloidal superball model as well, with the advantage of being visible with optical microscopy and being cubes rather than squares. Rossi et al. studied the crystallization of these colloidal cubes driven by depletion attraction into SC crystals ${ }^{[29]}$. They also revealed that three different types of crystal structures could be obtained depending on the depletion range and effective roundness of the particles $(2<m<3.8)^{[30]}$. Their result shows that the roundness of these cubes will indeed affect the crystal structures they can form. However, due to the added depletion attraction between the cubes it is hard to directly relate their findings to the predicted structures for hard superballs ${ }^{[73,75,76]}$. In this thesis we therefore want to investigate whether these hollow colloidal silica cubes will form close-packed structures in suspension and to compare the crystal structures that are obtained to those found in simulations.

\section{I.4. Colloidal crystal preparation}

Before introducing the content of this thesis in Section 1.6, we will first review the various methods for preparation in Section 1.4 and characterization in Section 1.5 of both sphere and cube colloidal crystals that play an essential role in this thesis. There are several ways to prepare colloidal crystals of which the most convenient methods are sedimentation by gravity or controlled evaporation of the solvent in air ${ }^{[85]}$ or in silicon oil ${ }^{[86]}$.

\section{I.4. I. Sedimentation}

The effect of the gravitational field on colloids dispersed in a fluid is described by the Stokes settling velocity;

$$
U_{0}=\frac{2}{9} \frac{R^{2} \Delta \rho}{\mu} g
$$

here $R$ is the radius of the particle, $\Delta \rho$ the buoyant density difference between the particle and the fluid, $\mu$ the fluid viscosity and $g$ the gravitational acceleration. The settling is counter balanced by the Brownian diffusion of the particles with thermal energy $k T$, where $k$ is the Boltzmann constant and $T$ the absolute temperature. A measure for this balance is the gravitational length, 


$$
L_{g}=\frac{k T}{M_{b} g}=\frac{3 k T}{4 \pi R^{3} \Delta \rho g}
$$

where $M_{b}$ is the buoyant mass. It is clear that $L_{g}$ strongly dependents on material via $\Delta \rho$ and the particle size, $R$. In practice this means that if $L_{g}$ is much larger than the container height $h$, there will be little effect of gravity on the particle distribution. In the other extreme, $\mathrm{h}>>L_{g^{\prime}}$ particles will settle quickly into dense sediments and crystallization might be hindered. For the practical fabrication of colloidal crystals by sedimentation this means that one should strive for a density difference $\Delta \rho$ and $h>L_{g}$ such that sedimentation will occur in the system on reasonable time scales.

Figure 1.5.a. shows a schematic representation of the sedimentation of colloids into a dense sediment in which crystallization can occur. This process can be tuned though full control over the crystal structure is hard to achieve. In part this is due to the limited control of the sedimentation speed of the particles: if this is too fast amorphous or glass structures will be formed. Furthermore, in the dense sediment colloidal crystal nuclei will form at different locations, which results in a polycrystalline structure with different domain sizes.

\section{I.4.2. Convective assembly}

In convective assembly (controlled drying) a dispersion containing a low concentration of monodisperse colloids is slowly evaporated on a substrate ${ }^{[85]}$. On the substrate the colloidal particles will self-assemble driven by capillary forces and convective flow. The capillary forces are extremely strong and lead to inter-particle interaction energies of thousands of $k T^{855}$. The convective flow is induced by the faster solvent evaporation at the thin solvent meniscus and causes transport of particles to the meniscus followed by the growth of close-packed structures. The technique has been applied as a bottom-up approach for the fabrication of novel functional nanomaterials, such as photonic crystals ${ }^{[5,59,87,88]}$.

Figure 1.5.b shows a schematic representation of the process for the most commonly used convective assembly setup, the vertical deposition (VD) setup. The VD setup was first introduced by Jiang et al. ${ }^{[89]}$, who fabricated multilayer single crystals on a substrate inserted vertically into the colloidal dispersion. Over the years the VD technique has been improved, for example, by the implementation of motorized stages for the substrate or the solvent container that allows control over the movement of the liquid-substrate interface ${ }^{[0,91]}$. Another useful method, with which the formation of crystals from large $(1 \mu \mathrm{m})$ colloidal silica spheres is possible, is the addition of a temperature gradient in the dispersion that prevents particle sedimentation ${ }^{[60]}$. The crystals formed with convective assembly show a tendency towards FCC stacking, which has inspired detailed studies of the assembly process ${ }^{[22-95]}$. Moreover, the need to prepare perfect defect-free crystals for photonic applications, resulted in the identification of different defect structures and their causes ${ }^{[2,296,97]}$, and has even led to control over defect incorporation ${ }^{[98-102]}$.

The thickness and uniformity of the colloidal crystals can be controlled by several experimental factors during the convective assembly process. The most important parameter 

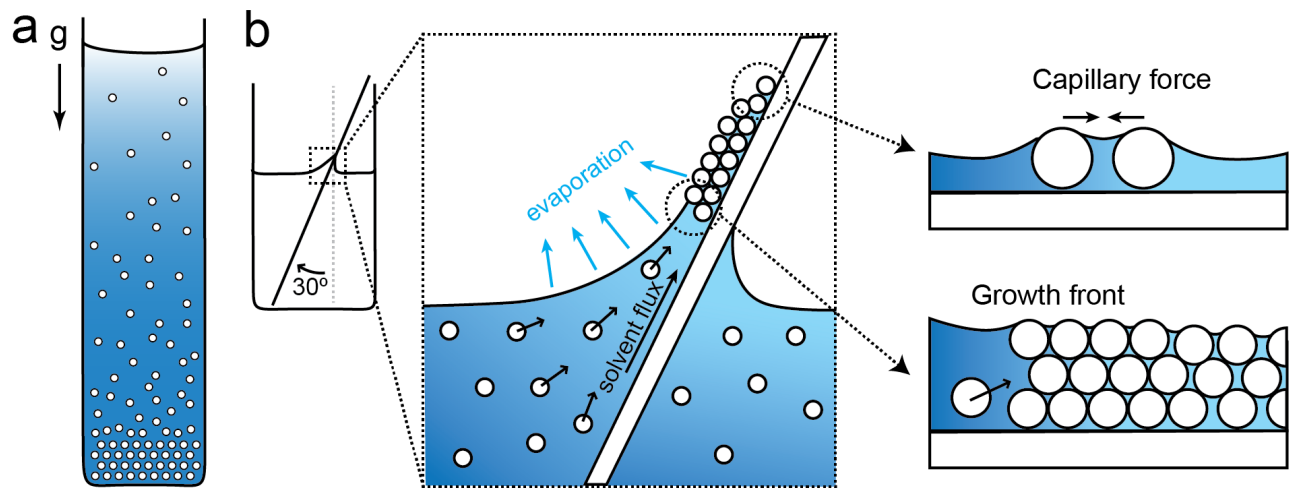

Figure 1.5. Schematic representation of a) sedimentation of a colloidal suspension into an ordered sediment and b) the convective assembly process, here shown for the vertical deposition setup. The evaporation of solvent causes a particle flux toward the meniscus where a close packed structure will form, due to capillary forces caused by the thin solvent film and solvent wetting of the particles. At the growth front particles will arrange into the niches of the growing structure.

is the particle flux that, similar to the sedimentation speed discussed above, determines if a crystalline structure will form. For a too high flux the particles get jammed at the growth front, while a too low flux will result in little or no deposition at all. An interesting example that demonstrates the control which can be achieved is the fabrication of banded crystal structure, that are created via a so-called stick-slip motion of the meniscus ${ }^{[103]}$. The tunable experimental parameters are listed below and are all connected and this should be kept in mind when doing convective assembly experiments.

- Particle size and material. Larger sizes and higher mass densities will cause faster sedimentation which can lead to depletion of particles close to the growth front.

- Volume fraction. The particle flux scales linearly with the volume fraction if other factors are kept constant.

- Solvent. The choice of solvent influences the speed of evaporation and contact angle of the meniscus.

- Substrate material and orientation. The chemical nature of the substrate as well as the angle the substrate makes with the suspension both also influence the meniscus shape.

- Temperature. An increase in temperature will enhance the evaporation speed of the solvent and the diffusion of the particles.

- Humidity and air flow. Both are linked to the evaporation speed of aqueous solvents and is the most challenging parameter to control experimentally. 


\section{I.5. Characterization of colloidal crystals}

Characterization of the 3D structure of colloidal crystals can be done in real or reciprocal space using a number of different experimental techniques. In this section the techniques used throughout this thesis are briefly outlined.

\section{I.5.I. Real space techniques}

Real space imaging reveals the local structures formed by colloidal particles and the particle orientation in case of anisotropic particle shapes. Common direct-space techniques for colloidal systems study are electron microscopy and optical (confocal) microscopy.

\section{Electron microscopy}

Electron microscopy (EM) is often used characterize the structure of materials. Due to the short wavelength of electrons a high resolution can be achieved. Both with transmission and scanning electron microscopy (TEM and SEM) detailed information is obtained about the particle composition, shape and the structures formed by self-organization. Two of the main drawbacks of EM are that; 1. the samples have to be dried before imaging and are exposed to a high vacuum, which can alter the sample composition; 2. Electrons have a short penetration depth and only surface of single particle information can be obtained.

\section{Confocal scanning laser microscopy}

The advantage of optical microscopy is that colloids can be directly imaged in suspension. Ordinary optical microscopy, however, can only be used to image a few layers of particles making it difficult to determine the real 3D structure. The resolution and depth of field can be improved by employing confocal scanning laser microscopy (CSLM). Initially used in biology, CSLM has proven to be a valuable tool for the imaging of local structures and dynamics in colloidal systems in-situ ${ }^{[104-106]}$.

Figure 1.6.a shows a schematic outline of the principle of the CSLM. By imaging a sample point by point and removing out of focus light an exceptionally short depth of field combined with an increased resolution is achieved. Single point illumination in the sample occurs through a source pinhole and an objective lens (black line). The emitted fluorescent light (grey line) of the sample is collected back through the objective, a dichroic mirror and a second pinhole, which is placed exactly in the conjugate focal (confocal) plane of the imaged spot. As a result all light (dashed line) that is not coming from the focal plane is filtered out. By lateral scanning of all focal points, a sharp 2D image of the focal plane can be obtained. By changing the focal depth, also $2 \mathrm{D}$ planes at other heights can be studied, resulting in the possibility to scan the sample in 3D. Figure 1.6.b. shows a typical confocal image obtained from a suspension of hollow silica cubes. The fluorescent shell can be clearly distinguished from the dark solvent which is also present in the hollow core of the cubes.

A drawback of optical microscopy is its limited resolution (at best 250-300 nm), which strongly reduces the range of applications. A partial solution is provided by several super-resolution 

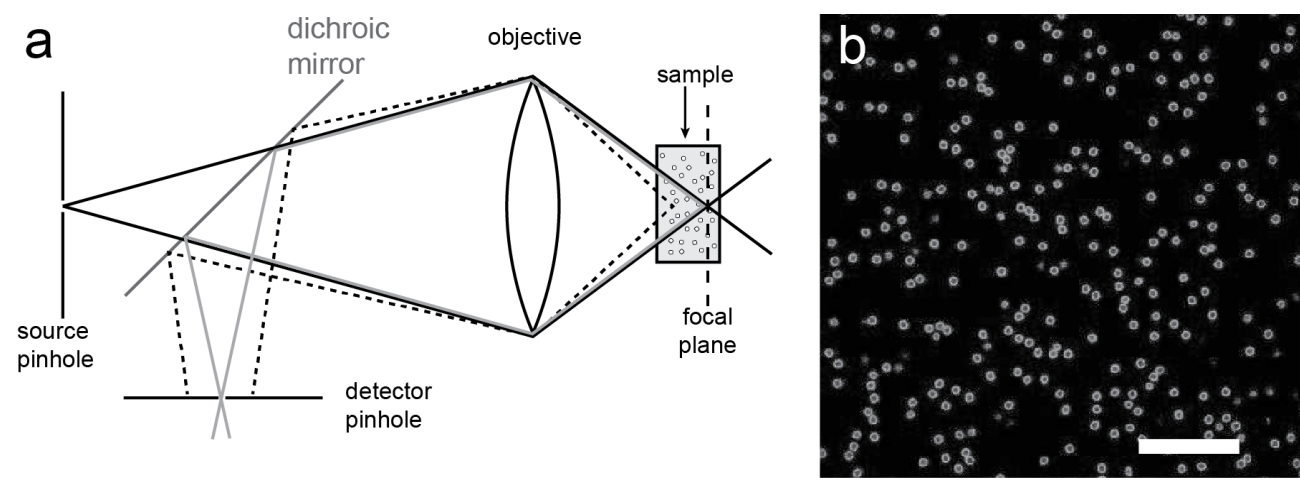

Figure I.6. a) Schematic illustration of the confocal microscope. The solid line represents the in-focus light, the dashed line represents the out-of-focus light. This is filtered out by the second pinhole in front of the detector. b) Typical confocal image obtained of fluorescent hollow silica cubes in suspension. Scale bar is $20 \mu \mathrm{m}$.

optical microscopy techniques that go beyond the diffraction limit ${ }^{[107,108]}$. However, CSLM additionally requires fluorescent labelling op the particles, high transparency of the samples and close matching of particle-solvent refractive indexes. The reason for this is that to be able to construct $3 \mathrm{D}$ images the fluorescence signal has to be obtained from within the sample. The refractive index match also conveniently decreases the van der Waals attractions between the colloids. Unfortunately, the CSLM requirements are difficult to meet for a wide range of colloidal systems, such as opaque samples or infiltrated photonic crystals.

\section{Transmission X-ray microscopy}

$\mathrm{X}$-ray microscopy is a relatively new technique that present a solution to some of the drawbacks of electron or optical microscopy. The shorter wavelength of X-rays lift restrictions related to diffraction limitations that are problematic in optical microscopy and their higher penetration power is advantageous in comparison with electron microscopy, especially for in-situ studies in colloidal suspensions ${ }^{[109-111]}$. Moreover, X-rays can be used for practically all materials and hence index matching or fluorescent labelling of the colloids is not needed.

An X-ray microscopy setup does, however, require specific optical elements as X-rays cannot be focused with traditional lenses. One option for focusing X-rays are Fresnel zone plates that can focus soft X-rays in a small spot. By scanning the sample with an X-ray energy close to the absorption edge of one of the sample elements an absorption contrast image can be constructed. This method is called scanning transmission X-ray microscopy (STXM) ${ }^{[112]}$. With modern Fresnel zone plates one can achieve element-specific imaging with a resolution on the order of about $10 \mathrm{~nm}^{[113]}$. Nevertheless, the penetration depth here is usually limited to tenths of micrometers, which is advantageous in comparison to electron microscopy but still insufficient for bulky, highly adsorbing samples.

In this thesis we use full field transmission X-ray microscopy (TXM) ${ }^{[114-116]}$ that allows imaging of thick (up to 200 microns) samples due to the high penetration depth of the hard 


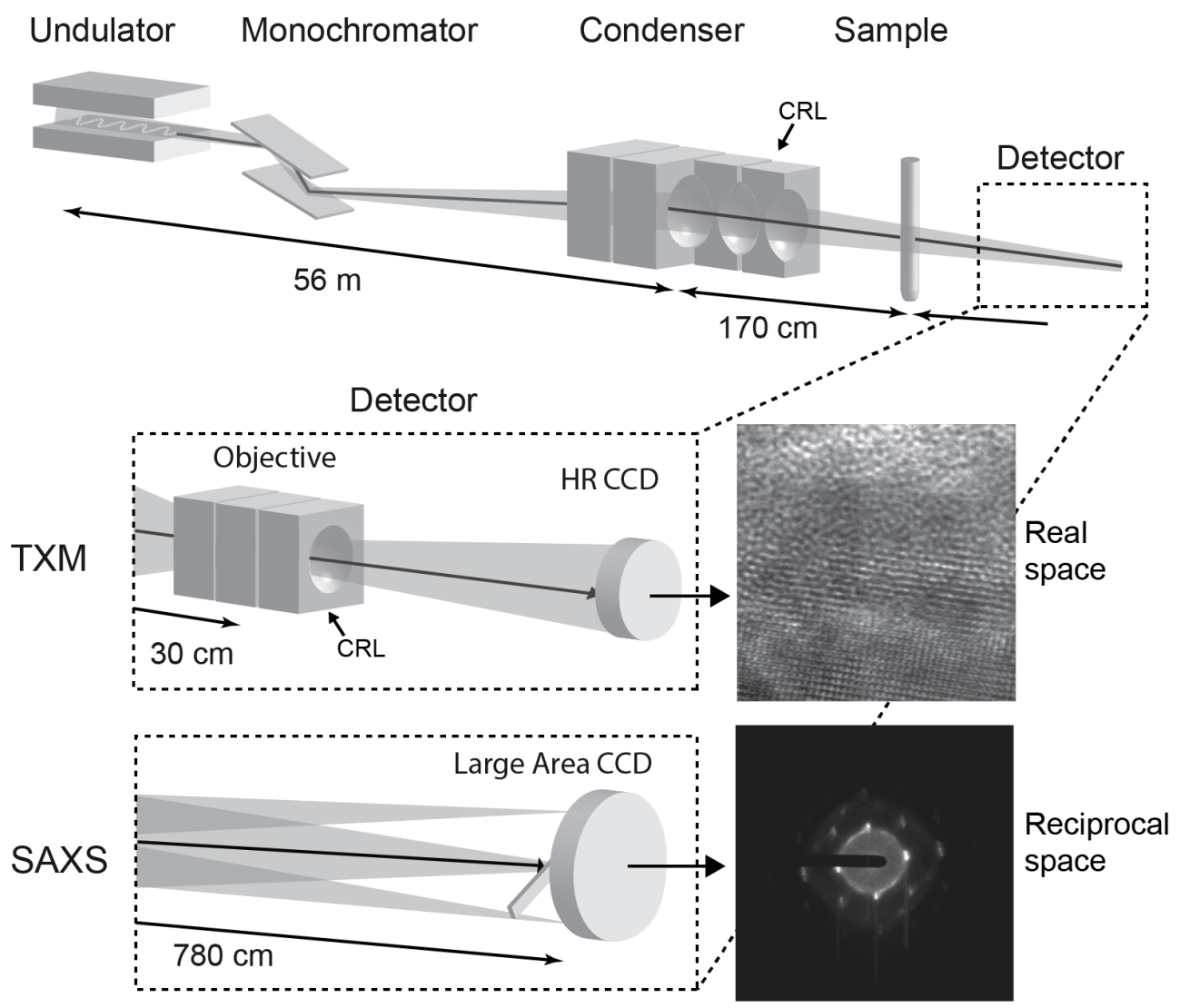

Figure 1.7. Schematic representation of the TXM and SAXS setup. In the TXM setup a second set of CRL is used as objective. In the SAXS setup the direct and scattered X-rays are focused on the detector. This figure in adapted from ref. [II7] and reproduced by permission of The Royal Society of Chemistry (RSC).

X-rays. Figure 1.7 shows a scheme of the TXM setup that consists of two sets of compound refractive lenses $(\mathrm{CRL})^{[117]}$ for beam focusing (condenser) and for projecting a magnified image of the sample on a 2D detector (objective). The setup can be easily switched from microscopy to diffraction mode (which will be explained in the next section) simply by moving the objective CRL out of the beam. The advantage of this setup is that that access to both real and reciprocal space yields complementary structural information.

\section{I.5.2. Reciprocal space}

Although real space techniques provide extremely valuable information on the local structure of colloidal crystals, one of the biggest drawbacks is the limited field of view. Different regions in the sample may be missed by chance and a small number of images leads to low structure statistics. In this respect well-established light and X-ray scattering techniques are superior, as these yield ensemble-averaged information in a large sample volume. Information on the 
long-range ordering in the sample can be readily obtained from the scattering data while that of the local structure is more difficult to gain. Therefore, microscopy and scattering are often used as complementary techniques.

\section{Small angle X-ray scattering}

Small angle X-ray scattering (SAXS) provides insight into the characteristics of colloidal suspensions on the spatial correlations and long-range order in crystals but also in terms of averaged particle size and shape $\mathrm{e}^{[22,42,118-121]}$. The bottom part of Figure 1.7 shows a schematic representation of the SAXS setup. An X-ray beam passes through the sample and is focused with CRLs on the detector, usually a CCD camera, where a scattering pattern is obtained. The scattering pattern can be represented as a function of the scattering vector $q$, of which the magnitude for elastic scattering is given by:

$$
q=\frac{4 \pi}{\lambda} \sin (\theta)
$$

here $\lambda$ is the wavelength and $2 \theta$ the scattering angle. Because the size of colloidal particles $(1 \mu \mathrm{m})$ is much larger than the wavelength of X-rays $(\sim 1 \AA)$, the scattering angles are very small. To resolve scattering at such small angles, a microradian X-ray diffraction setup was often used in this thesis ${ }^{[119,120]}$.

The scattering patterns of colloidal crystals contain two distinct features that arise from the size and shape of the particles and from the structure or spatial configuration of the particles. The scattered intensity $I(q)$ is directly related to the contributions of the particles described by the particle form factor $P(q)$ and their spatial correlations described by the structure factor $S(q)$. For spherical particles there is a simple relation ${ }^{[122]}$ :

$$
I(\vec{q}) \propto P(q) S(\vec{q})
$$

Note here that the $P(q)$ contribution of a sphere is homogeneous in all directions, while for particles of a more complex shape this relation does not hold because of the anisotropic form factor and the coupling between orientational and translational correlations. We will, however, in Part B of this thesis use Eq.(1.5) to approximate certain features observed in the scattering patterns from crystalline structures consisting of cubes.

Figure 1.8 shows schematic illustrations of the 2D SAXS patterns of differently ordered phases in colloidal suspensions. Disordered or fluid phases, as well as crystal powders, possess local structure, i.e. correlations among the relative positions of particles that persist over short distances (of the order of the particle size). In the scattering pattern this will result in concentric circles around the direct X-ray beam, represented by the white circle in Figure 1.8.a. Partially oriented samples, such as liquid crystals or samples under shear will result in intensity modulations along this circle (Fig. 1.8.b). In contrast, ordered or crystal phases possess periodic structures over a long range (many particle radii) and result in intense spots (Bragg reflections) 

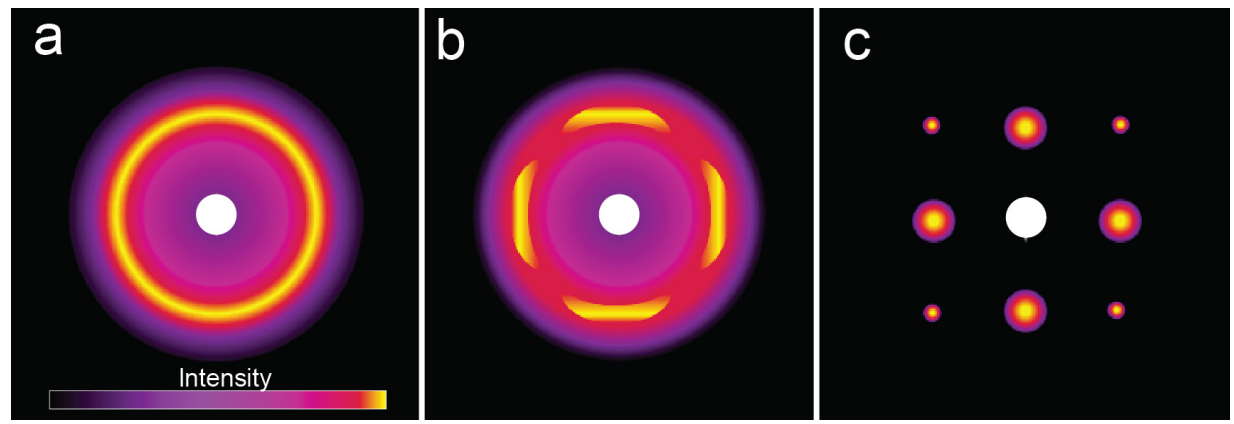

Figure I.8. Schematic 2D scattering patterns around direct beam (white circle) of a) randomly oriented (isotropic), b) partially oriented and c) perfectly oriented (single crystal) samples.

in the SAXS pattern at specifc $q$ (Fig.1.8.c).

\section{Coherent X-ray Diffraction Imaging}

Coherent diffraction imaging (CXDI) is a relatively novel "lensless" imaging technique that relies on phase contrast rather than absorption contrast ${ }^{[123-126]}$. It consists of measuring the diffraction pattern of a finite-sized object that is illuminated with a coherent X-ray beam. CXDI has been applied to the imaging of different samples and (crystalline) materials ${ }^{[124,126]}$ as well as colloidal crystals ${ }^{[127,128]}$. The use of a coherent X-ray beam results in the appearance of distinct features between the Bragg reflections that are not present in a typical SAXS pattern and are directly related the size and shape of the studied object. By sufficiently sampling the diffraction pattern it can be converted to a real space image using iterative phase retrieval methods ${ }^{[125,129,130]}$. One of the drawbacks of CXDI is that it requires finite-sized sample objects and the real-space image reconstruction involves complex algorithms with still limited reliability.

\section{I.6. Outline of this thesis}

This thesis focuses on ordered systems of colloidal particles of two shapes: spheres and cubes. Correspondingly the thesis is split into two parts, Part A - Spheres and Part B - Cubes.

In Part A we focus on two different types of colloidal crystals, dried colloidal crystals and colloidal crystals in suspension. We start in Chapter 2 with the preparation of these dried colloidal crystals via sedimentation and vertical deposition. Our aim is to investigate the structure and possible defects via several types of X-ray diffraction experiments performed at synchrotrons and free electron lasers, which allow larger sample volumes and provides better statistics on the structure analysis. However, each X-ray diffraction setup has specific sample requirements which have to be met and the experimental challenges are described. In addition, the results of two experiments - structure evolution upon heating or induced by ultrashort laser pulses - are presented. Next we study in Chapter 3 one of the specially prepared sedimentary colloidal crystals grains on a pin with coherent X-ray diffraction imaging (CXDI). The local crystal lattice structure was investigated via means of Bragg Rod analysis, a diffuse 
scattering feature that is related to the presence of stacking defects. In addition, the preliminary result from the full real-space reconstruction, the ultimate goal of CDXI experiments, of the crystal grain is presented. In Chapter 4 we turn our attention to colloidal crystals in suspension, where particle dynamics can still occur, in order to study defect diffusion, as subject that has received limited attention. For this we use microgel spheres of poly-N-isopropyl acrylamide (PNIPAM) that are thermo-responsive allowing one to tune their size with temperature. By labeling the particles with a fluorescent dye we can study the crystals and defects in 3D with confocal scanning laser microscopy (CSLM). We explore if defect structures can be induced by embedding these PNIPAM spheres in a non-thermo-responsive crystal by means of changing the temperature, while imaging the structure and the dynamics in real-space and time.

Having completed the study of sphere crystals in Part A, we turn our attention to cube crystals in Part B. Our first task here is obviously the preparation of colloidal cubes in Chapter 5. We employ the method introduced in ref. [28] comprising of the synthesis of a hematite cube. This cube can be subsequently coated with silica and the core can be dissolved as introduced in ref. [29] to result in hollow silica cubes. Our aim in this part of the thesis is to investigate the role of the rounded cube shape on the close-packed structures that the cubes will form. Therefore, we study in Chapter 6 crystalline structures formed by these hollow colloidal silica cubes via convective assembly. The experimental conditions are tuned and the structures are characterized in detail with scanning electron microscopy (SEM) and small angle X-ray scattering (SAXS). We show that due to their rounded shape and the close-packed conditions induced during crystal growth, the cubes form the two optimal packings $\Lambda_{0}$ and $\Lambda_{1}$-lattice as predicted by simulations. In addition, we increase the cube $m$-value in Chapter 7 as the simulations also predict that a small change in $m$ might drive the crystal formation in favor of the $\Lambda_{1}$-lattice. In this chapter we focus on the cubes ordered into monolayers and quantify the $2 \mathrm{D}$ structures using computer analysis of the local particle environment. We find conclusive evidence that two predicted optimal structures the $\Lambda_{0}$ and $\Lambda_{1}$-lattices are formed with a dominance of the $\Lambda_{1}$-lattice. In Chapter 8 we investigate the assembly of the charge stabilized hollow colloidal silica cubes in suspension and to what extend the Debye screening length range effects their self-assembly. This is motivated by the knowledge that systems composed of particles with purely repulsive interactions are expected to assemble into ordered phases driven by purely entropic interactions alone. Due to the size of the cubes $(\sim 1 \mu \mathrm{m})$ sedimentation under gravity is unavoidable and we investigate the dense crystalline sediments that are obtained in detail with small angle X-ray scattering (SAXS). In Chapter 9 we turn our attention to the hematite cubes that possess a permanent magnetic dipole moment and focus on the characterization of the formed sedimentary crystals with SAXS. The effect of the dipole-dipole attractions on the structures under different solvent and salt conditions are investigated. Also, the alignment of the cubes is induced by an external magnetic field and we characterize in detail the single crystal structure of cubes that spontaneously forms under these conditions. 


\section{Acknowledgements}

Roel Baars is acknowledged for kindly providing the 3D images of the superballs.

\section{References}

1. International Union of Pure and Applied Chemistry http://goldbook.iupac.org/C01172, September 2014.

2. L. Onsager, Chem. Rev., 1933, 13, 73-89.

3. W. G. McMillan and J. E. Mayer, J. Chem. Phys., 1945, 13, 276-305.

4. J. N. Israelachvili, Intermolecular and Surface forces, Academic Press, Inc., 1985.

5. D. F. Evans and H. Wennerström, The colloidal domain: where physics, chemistry, biology and technology meet, Wiley-VCH, New York, 1999.

6. R. J. Hunter, Foundations of colloid science, Oxford University Press, Oxford, 2001.

7. H. N. W. Lekkerkerker and R. Tuinier, Colloids and the depletion interaction, Springer, 2011.

8. J. Perrin, Annales De Chimie Et De Physique, 1909, 18, 5-114.

9. W. Poon, P. N. Pusey and H. N. W. Lekkerkerker, Physics World, 1996, 9, 27-32.

10. W. Poon, Science, 2004, 304, 830-831.

11. J. G. Kirkwood, J. Chem. Phys., 1939, 7, 919-925.

12. J. G. Kirkwood and E. Monroe, J. Chem. Phys., 1941, 9, 514-526.

13. L. Onsager, Ann. NY. Acad. Sci., 1949, 51, 627-659.

14. B. J. Alder and T. E. Wainwright, J. Chem. Phys., 1957, 27, 1208-1209.

15. W. W. Wood and J. D. Jacobson, J. Chem. Phys., 1957, 27, 1207-1208.

16. W. G. Hoover and F. H. Ree, J. Chem. Phys., 1968, 49, 3609-3617.

17. J. B. Jones, E. R. Segnit and J. V. Sanders, Nature, 1964, 204, 994.

18. S. H. Cole and E. A. Monroe, J. Appl. Phys., 1967, 38, 1872-1873.

19. P. N. Pusey, W. van Megen, P. Bartlett, B. J. Ackerson, J. G. Rarity and S. M. Underwood, Phys. Rev. Lett., 1989, 63, 2753-2756.

20. A. Yethiraj and A. van Blaaderen, Nature, 2003, 421, 513-517.

21. F. Marlow, P. Sharifi, R. Brinkmann and C. Mendive, Angew. Chem., 2009, 48, 6212-33.

22. F. M. van der Kooij, K. Kassapidou and H. N. W. Lekkerkerker, Nature, 2000, 406, 868-871.

23. E. van den Pol, A. V. Petukhov, D. M. E. Thies-Weesie, D. V. Byelov and G. J. Vroege, Phys. Rev. Lett., 2009, 103, 258301.

24. E. van den Pol, A. V. Petukhov, D. M. E. Thies-Weesie, D. V. Byelov and G. J. Vroege, J. Colloid Interface Sci., 2010, $352,354-358$.

25. I. D. Hosein, B. S. John, S. H. Lee, F. A. Escobedo and C. M. Liddell, J. Mater. Chem., 2009, 19, 344-349.

26. A. F. Demirors, P. M. Johnson, C. M. van Kats, A. van Blaaderen and A. Imhof, Langmuir, 2010, 26, 14466-14471.

27. D. J. Kraft, R. Ni, F. Smallenburg, M. Hermes, K. Yoon, D. A. Weitz, A. van Blaaderen, J. Groenewold, M. Dijkstra and W. K. Kegel, Proc. Natl. Acad. Sci. U. S. A., 2012, 109, 10787-10792.

28. T. Sugimoto and K. Sakata, J. Colloid Interface Sci., 1992, 152, 587-590.

29. L. Rossi, S. Sacanna, W. T. M. Irvine, P. M. Chaikin, D. J. Pine and A. P. Philipse, Soft Matter, 2011, 7, 4139-4139.

30. L. Rossi, Colloidal Superballs, Ph.D. Thesis, Utrecht University, 2012.

31. Y. Sun and Y. Xia, Science, 2002, 298, 2176-2179.

32. I. Sevonkaev, D. V. Goia and E. Matijević, J. Colloid Interface Sci., 2008, 317, 130-136.

33. T. Sugimoto, M. M. Khan, A. Muramatsu and H. Itoh, Colloids Surf. A., 1993, 79, 233-247.

34. S. H. Lee and C. M. Liddell, Small, 2009, 5, 1957-1962.

35. Y. Xia, Y. Xiong, B. Lim and S. E. Skrabalak, Angew. Chem. Int. Ed., 2009, 48, 60-103.

36. Z. Zhu, H. Meng, W. Liu, X. Liu, J. Gong, X. Qiu, L. Jiang, D. Wang and Z. Tang, Angew. Chem., 2011, 50, 1593-1596.

37. J. Henzie, M. Grünwald, A. Widmer-Cooper, P. L. Geissler and P. Yang, Nature Mater., 2012, 11, 131-137.

38. S. C. Glotzer and M. J. Solomon, Nature Mater., 2007, 6, 557-562.

39. P. F. Damasceno, M. Engel and S. C. Glotzer, Science, 2012, 337, 453-457.

40. J. Hilhorst and A. V. Petukhov, Phys. Rev. Lett., 2011, 107, 095501.

41. J. Hilhorst, M. M. van Schooneveld, J. Wang, E. de Smit, T. Tyliszczak, J. Raabe, A. P. Hitchcock, M. Obst, F. M. F. de Groot and A. V. Petukhov, Langmuir, 2012, 28, 3614-3620. 
42. J. Hilhorst, V. V. Abramova, A. Sinitskii, N. A. Sapoletova, K. S. Napolskii, A. A. Eliseev, D. V. Byelov, N. A. Grigoryeva, A. V. Vasilieva, W. G. Bouwman, K. Kvashnina, A. Snigirev, S. V. Grigoriev and A. V. Petukhov, Langmuir, 2009, 25, 10408-10412.

43. H. R. Vutukuri, Complex Colloidal Structures by Self-assembly in Electric Fields, Ph.D. Thesis, Utrecht University, 2012.

44. T. Alfrey Jr., E. B. Bradford, J. W. Vanderhoff and G. Oster, J. Opt. Soc. Am., 1954, 44, 603-607.

45. A. K. Van Helden, J. W. Jansen and A. Vrij, J. Colloid Interface Sci., 1981, 81, 354-368.

46. L. Antl, J. W. Goodwin, R. D. Hill, R. H. Ottewill, S. M. Owens, S. Papworth and J. A. Waters, Colloids Surf., 1986, 17, 67-78.

47. P. N. Pusey and W. van Megen, Nature, 1986, 320, 340-342.

48. C. G. De Kruif, P. W. Rouw, J. W. Jansen and A. Vrij, J. Physique, 1985, 46, 295-308.

49. J. Zhu, M. Li, R. Rogers, W. Meyer, R. Ottewill, W. Russell and P. Chaikin, Nature, 1997, 387, 883-885.

50. W. Kegel and J. Dhont, J. Chem. Phys., 2000, 112, 3431-3436.

51. A. V. Petukhov, I. P. Dolbnya, D. G. A. L. Aarts, G. J. Vroege and H. N. W. Lekkerkerker, Phys. Rev. Lett., 2003, 90, 028304.

52. I. P. Dolbnya, A. V. Petukhov, D. G. A. L. Aarts, G. J. Vroege and H. N. W. Lekkerkerker, EPL, 2005, 72, 962-968.

53. J. H. J. Thijssen, A. V. Petukhov, D. C. 't Hart, A. Imhof, C. H. M. van der Werf, R. E. I. Schropp and A. van Blaaderen, Adv. Mater., 2006, 18, 1662-1666.

54. U. Gasser, E. R. Weeks, A. Schofield, P. N. Pusey and D. A. Weitz, Science, 2001, 292, 258-262.

55. V. W. A. de Villeneuve, R. P. A. Dullens, D. G. A. L. Aarts, E. Groeneveld, J. H. Scherff, W. K. Kegel and H. N. W. Lekkerkerker, Science, 2005, 309, 1231-1233.

56. A. M. Alsayed, M. F. Islam, J. Zhang, P. J. Collings and A. G. Yodh, Science, 2005, 309, 1207-1210.

57. Y. Peng, Z. Wang, A. M. Alsayed, A. G. Yodh and Y. Han, Phys. Rev. Lett., 2010, 104, 205703.

58. J. E. G. J. Wijnhoven and W. L. Vos, Science, 1998, 281, 802-804.

59. A. C. Blanco E., S. Grabtchak, M. Ibisate, S. John, S. Leonard, C. Lopez, F. Meseguer, H. Miguez, J. Mondia, G. Ozin, O. Toader and H. M. van Driel, Nature, 2000, 405, 437-40.

60. Y. Vlasov, X. Z. Bo, J. C. Sturm and D. J. Norris, Nature, 2001, 414, 289-93.

61. A. Hynninen, J. H. J. Thijssen, E. C. M. Vermolen, M. Dijkstra and A. van Blaaderen, Nature Mater., 2007, 6, 202-205.

62. P. Schall, I. Cohen, D. A. Weitz and F. Spaepen, Science, 2004, 305, 1944-1948.

63. C. Eisenmann, U. Gasser, P. Keim, G. Maret and H. H. von Grunberg, Phys. Rev. Lett., 2005, 95, 185502.

64. J. M. Meijer, V. W. A. de Villeneuve and A. V. Petukhov, Langmuir, 2007, 23, 3554-3560.

65. W. Lechner and C. Dellago, Soft Matter, 2009, 5, 2752-2758.

66. W. Lechner, D. Polster, G. Maret, P. Keim and C. Dellago, Phys. Rev. E, 2013, 88, 060402.

67. A. Guinier, X-Ray Diffraction in Crystal, Imperfect Crystals, and Amorphous Bodies, Dover, New York, 1994.

68. P. G. Bolhuis, D. Frenkel, S. C. Mau and D. A. Huse, Nature, 1997, 388, 235-236.

69. Y. Zhang, F. Lu, D. van der Lelie and O. Gang, Phys. Rev. Lett., 2011, 107, 135701.

70. H. Yang, S. He, H. Chen and H. Tuan, Chem. Mater., 2014, 26, 1785-1793.

71. A. Ahniyaz, Y. Sakamoto and L. Bergstrom, Proc. Natl. Acad. Sci. U. S. A., 2007, 104, 17570-17574.

72. S. Disch, E. Wetterskog, R. P. Hermann, G. Salazar-Alvarez, P. Busch, T. Brueckel, L. Bergstroem and S. Kamali, Nano Lett., 2011, 11, 1651-1656.

73. Y. Jiao, F. H. Stillinger and S. Torquato, Phys. Rev. E, 2009, 79, 041309.

74. Y. Jiao, F. H. Stillinger and S. Torquato, Phys. Rev. Lett., 2008, 100, 245504.

75. R. D. Batten, F. H. Stillinger and S. Torquato, Physical Review E, 2010, 81, 1-13.

76. R. Ni, A. P. Gantapara, J. de Graaf, R. van Roij and M. Dijkstra, Soft Matter, 2012, 8, 12135-12135.

77. C. Avendano and F. A. Escobedo, Soft Matter, 2012, 8, 4675-4681.

78. M. Marechal, U. Zimmermann and H. Loewen, J. Chem. Phys., 2012, 136, 144506.

79. F. Smallenburg, L. Filion, M. Marechal and M. Dijkstra, Proc. Natl. Acad. Sci. U. S. A., 2012, 109, 17886-17890.

80. E. A. Jagla, Phys Rev E., 1998, 58, 4701-4705.

81. K. W. Wojciechowski and D. Frenkel, Comp. Met. Sci. Technol., 2004, 10, 235-255.

82. B. Groh and B. Mulder, J. Chem. Phys., 2001, 114, 3653-3658.

83. S. Belli, M. Dijkstra and R. van Roij, J. Chem. Phys., 2012, 137, 124506.

84. K. Zhao, R. Bruinsma and T. G. Mason, Proc. Natl. Acad. Sci. U. S. A., 2011, 108, 2684-2687.

85. N. Denkov, O. Velev, P. Kralchevski, I. Ivanov, H. Yoshimura and K. Nagayama, Langmuir, 1992, 8, 3183-3190. 86. A. P. Philipse, J. Mater. Sci. Lett., 1989, 8, 1371-1373. 
87. H. Miguez, F. Meseguer, C. Lopez, A. Blanco, J. S. Moya, J. Requena, A. Mifsud and V. Fornes, Adv. Mater., 1998, 10, 480-483.

88. J. F. Galisteo-Lopez, M. Ibisate, R. Sapienza, L. S. Froufe-Perez, A. Blanco and C. Lopez, Adv. Mater., 2011, 23, 30-69.

89. P. Jiang, J. F. Bertone, K. S. Hwang and V. L. Colvin, Chem. Mater., 1999, 11, 2132-2140.

90. J. Zhang, Z. Sun and B. Yang, Curr. Opin. Colloid Interface Sci., 2009, 14, 103-114.

91. Z. Zhou and X. S. Zhao, Langmuir, 2005, 21, 4717-23.

92. S. H. Im, M. H. Kim and O. O. Park, Chem. Mater., 2003, 15, 1797-1802.

93. L. Meng, H. Wei, A. Nagel, B. J. Wiley, L. E. Scriven and D. J. Norris, Nano Lett., 2006, 6, 2249-53.

94. D. D. Brewer, J. Allen, M. R. Miller, J. M. D. Santos, S. Kumar, D. J. Norris, M. Tsapatsis and L. E. Scriven, Langmuir, 2008.

95. H. Cao, D. Lan, Y. Wang, A. Volinsky, L. Duan and H. Jiang, Phys. Rev. E, 2010, 82, 1-6.

96. Y. Ye, F. LeBlanc, A. Hache and V. Truong, Appl. Phys. Lett., 2001, 78, 52-52.

97. A. Hartsuiker and W. L. Vos, Langmuir, 2008, 24, 4670-5.

98. S. Kuai, X. Hu, a. Hache and V. Truong, J. Cryst. Growth, 2004, 267, 317-324.

99. L. Wang and X. S. Zhao, J. Phys. Chem. C, 2007, 111, 8538-8542.

100. W. Khunsin, G. Kocher, S. G. Romanov and C. M. S. Torres, Adv. Funct. Mater., 2008, 18, 2471-2479.

101. B. Hatton, L. Mishchenko, S. Davis, K. H. Sandhage and J. Aizenberg, Proc. Natl. Acad. Sci. U. S. A., 2010, 107, 10354-9.

102. K. S. Napolskii, N. A. Sapoletova, D. F. Gorozhankin, A. A. Eliseev, D. Y. Chernyshov, D. V. Byelov, N. A. Grigoryeva, A. A. Mistonov, W. G. Bouwman, K. O. Kvashnina, A. V. Lukashin, A. A. Snigirev, A. V. Vassilieva, S. V. Grigoriev and A. V. Petukhov, Langmuir, 2010, 26, 2346-2351.

103. J. Marqués-Hueso and H. J. Schöpe, Progr Colloid Polym Sci, 2008, 48-56.

104. A. Van Blaaderen, A. Imhof, W. Hage and A. Vrij, Langmuir, 1992, 8, 1514-1517.

105. A. van Blaaderen and P. Wiltzius, Science, 1995, 270, 1177-1179.

106. V. Prasad, D. Semwogerere and E. R. Weeks, J. Phys. : Condens. Matter, 2007, 19, 113102.

107. S. W. Hell, Science, 2007, 316, 1153-1158.

108. S. W. Hell, Nat. Methods, 2009, 6, 24-32.

109. K. Kaznacheev and T. Hegmann, Phys. Chem. Chem. Phys., 2007, 9, 1705-1712.

110. J. Thieme, I. McNulty, S. Vogt and D. Paterson, Environ. Sci. Technol., 2007, 41, 6885-6889.

111. S. Brisard, R. S. Chae, I. Bihannic, L. Michot, P. Guttmann, J. Thieme, G. Schneider, P. J. M. Monteiro and P. Levitz, Am. Mineral., 2012, 97, 480-483.

112. T. Warwick, K. Franck, J. Kortright, G. Meigs, M. Moronne, S. Myneni, E. Rotenberg, S. Seal, W. Steele, H. Ade, A. Garcia, S. Cerasari, J. Delinger, S. Hayakawa, A. Hitchcock, T. Tyliszczak, J. Kikuma, E. Rightor, H. Shin and B. Tonner, Rev. Sci. Instrum., 1998, 69, 2964-2973.

113. W. Chao, P. Fischer, T. Tyliszczak, S. Rekawa, E. Anderson and P. Naulleau, Opt. Express, 2012, 20, 9777-9783.

114. B. Lengeler, C. Schroer, J. Tummler, B. Benner, M. Richwin, A. Snigirev, I. Snigireva and M. Drakopoulos, J. Synchrotron Rad., 1999, 6, 1153-1167.

115. A. Bosak, I. Snigireva, K. S. Napolskii and A. Snigirev, Adv Mater, 2010, 22, 3256-3259.

116. D. V. Byelov, J. M. Meijer, I. Snigireva, A. Snigirev, L. Rossi, E. van den Pol, A. Kuijk, A. Philipse, A. Imhof, A. van Blaaderen, G. J. Vroege and A. V. Petukhov, RCS Advances, 2013, 3, 15670-15677.

117. A. Snigirev, V. Kohn, I. Snigireva and B. Lengeler, Nature, 1996, 384, 49-51.

118. W. Vos, M. Megens, C. vanKats and P. Bosecke, Langmuir, 1997, 13, 6004-6008.

119. J. H. J. Thijssen, A. V. Petukhov, D. C. 't Hart, A. Imhof, C. H. M. van der Werf, R. E. I. Schropp and A. van Blaaderen, Adv Mater, 2006, 18, 1662-1666.

120. A. Petukhov, J. Thijssen, D. t Hart, A. Imhof, A. van Blaaderen, I. Dolbnya, A. Snigirev, A. Moussaid and I. Snigireva, J. Appl. Cryst., 2006, 39, 137-144.

121. N. Grigoryeva, a. Mistonov, K. Napolskii, N. Sapoletova, a. Eliseev, W. Bouwman, D. Byelov, a. Petukhov, D. Chernyshov, H. Eckerlebe, a. Vasilieva and S. Grigoriev, Phys. Rev. B, 2011, 84, 1-13.

122. P. N. Pusey, Introduction to Scattering Experiments, ed. P. Lindner and T. Zemb, Elsevier Science b.v., Amsterdam, The Netherlands, 2002, pp.3-21.

123. I. K. Robinson, I. A. Vartanyants, G. J. Williams, M. A. Pfeifer and J. A. Pitney, Phys. Rev. Lett., 2001, 87, 195505.

124. I. Robinson and R. Harder, Nature Mater., 2009, 8, 291-298. 
125. K. A. Nugent, Adv. Phys., 2010, 59, 1-99.

126. A. P. Mancuso, O. M. Yefanov and I. A. Vartanyants, J. Biotechnol., 2010, 149, 229-237.

127. J. Gulden, O. M. Yefanov, A. P. Mancuso, V. V. Abramova, J. Hilhorst, D. Byelov, I. Snigireva, A. Snigirev, A. V. Petukhov and I. A. Vartanyants, Phys. Rev. B, 2010, 81, 224105.

128. J. Gulden, O. M. Yefanov, A. P. Mancuso, R. Dronyak, A. Singer, V. Bernatova, A. Burkhardt, O. Polozhentsev, A. Soldatov, M. Sprung and I. A. Vartanyants, Opt. Express, 2012, 20, 4039-4049.

129. J. R. Fienup, Appl. Opt., 1982, 21, 2758-2769.

130. V. Elser, JOSA A, 2003, 20, 40-55. 


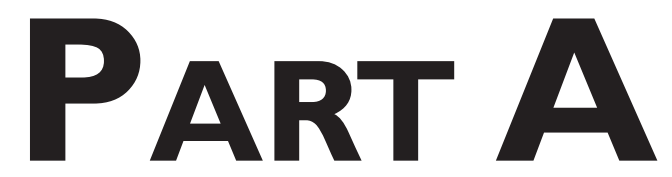

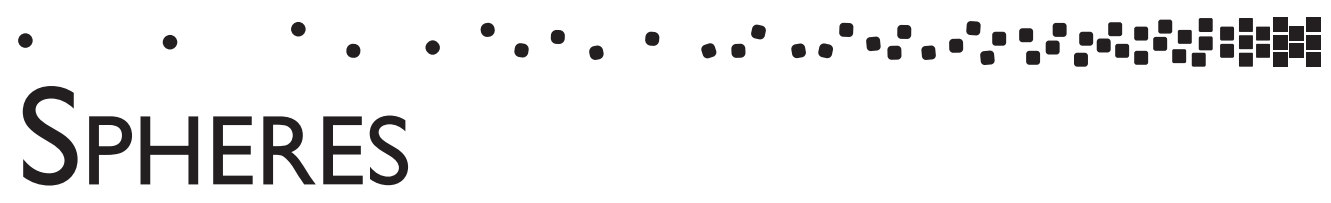




\section{ChAPTER 2}

$\bullet$

$\bullet$

$\bullet \bullet$

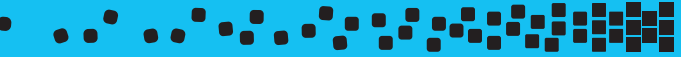

Preparation and Characterization OF COLLOIDAL CRYSTALS FOR SYNCHROTRON and Free Electron LASER X-RAY StUdies

Abstract

In this chapter we describe the preparation and characterization of colloidal crystals for dedicated synchrotron and free-electron laser X-ray studies. The crystals were prepared via sedimentation and convective assembly followed by modifications for their application in the experiments. We illustrate two examples of the $\mathrm{X}$-ray experiments, including the pump-probe experiments on the dynamics of laser heating of the colloidal particles on the crystal structure and structure melting around the glass transition temperature. 


\section{I. Introduction}

Colloidal crystals and their internal structure have been the focus of many investigations because of their possible application as functional nanomaterials, for instance as photonic crystals. ${ }^{[1-3]}$ The presence of defects in the crystal structure influences the functional properties of the colloidal crystal and therefore it is important to fully characterize both the crystal and defect structures in detail. Furthermore, investigations of the optical colloidal crystal properties, e.g. response of the photonic structure to strong optical fields, are important for technical feasibility of the crystals as photonic devices.

The improvement of $3^{\text {rd }}$ generation X-ray sources has led to the development of novel X-ray techniques, such as hard and soft X-ray microscopy ${ }^{[4-6]}$ as well as coherent X-ray diffraction imaging $(\mathrm{CXDI})^{[7]}$. These methods can be applied to reveal the internal structure of the colloidal crystals $^{[8] .}$ In addition, the colloidal crystals are challenging model systems to test the CXDI data analysis, which involves complex iterative phase retrieval methods to reconstruct the real space image ${ }^{[9-11]}$.

While the novel X-ray diffraction techniques offer new insights, they also demand stringent requirements for the sample preparation. In this chapter we describe the preparation of colloidal crystals from polystyrene and silica spheres via sedimentation and convective assembly that were used for different types of X-ray studies. The aspects of preparation are discussed in detail and the obtained structures are characterized using microscopy techniques. The outline of this chapter is as follows. First, we discuss the experimental details of the preparation. Next, the optimal conditions to fabricate large scale single colloidal crystals via the vertical deposition method are discussed and the crystals characterized. This is followed by a discussion on the preparation and difficulties to obtain $\sim 5 \mu \mathrm{m}$ colloidal crystal grains on a carbon fiber tip, which is needed to meet the sample requirements for a CXDI experiment. Finally, the results of two $\mathrm{X}$-ray diffraction studies performed of these samples are briefly discussed.

\subsection{Experimental}

\subsubsection{Colloidal spheres}

Several different colloidal sphere dispersions were used, with an average radius in the range of $R=50-300 \mathrm{~nm}$ and composed of either polystyrene or silica. The polystyrene particles were prepared by emulsifier-free emulsion polymerization of styrene using potassium persulfate as an initiator and are therefore charge stabilized by sulfate groups on their surface. The silica particles were prepared via the Stöber synthesis and were coated with 3-methacryloxypropyl trimethoxysilane (TPM) ${ }^{[12 \mathrm{a}]}$ or stearyl ${ }^{[12 \mathrm{~b}]}$. The size, radius $R$, and standard deviation, $\sigma$, of the particles was determined with transmission electron microscopy (TEM, Philips TECNAI10 or 12). For all particles used in this chapter the properties are listed in Table 2.1.

\subsubsection{Colloidal crystal preparation}

Colloidal crystal films were prepared on different substrates via the vertical deposition method. Polystyrene and sterically stabilized silica spheres were dispersed in water, ethanol 
Table 2.I. Particle properties of all colloidal spheres and type of experiment they were employed in.

\begin{tabular}{l|cccc}
\hline \multicolumn{1}{c|}{ Particle } & Material & $\begin{array}{c}\boldsymbol{R} \\
(\mathbf{n m})\end{array}$ & $\begin{array}{c}\boldsymbol{\sigma} \\
(\%)\end{array}$ & Experiment \\
\hline PS1 & Polystyrene & 208 & 2.1 & VD \\
PS2 & Polystyrene & 82 & 2.7 & VD \\
PS3 & Polystyrene & 193 & 2.1 & VD \\
CPS1 & Crosslinked (3\%) & 97 & 4 & VD \\
& polystyrene & & & VD \\
PS-R-B1271 $^{*}$ & Polystyrene & 180 & 2.3 & VD \\
BVR-I3 $^{*}$ Crosslinked (3\%) & 136 & 4 & Sed \\
DB239* $^{* *}$ & polystyrene & & & Sed,VD \\
DB275 $^{* *}$ & Silica-TPM & 100 & - & Sed \\
DB284 $^{* *}$ & Silica-TPM & 118 & 6 & Sed \\
DB359** $^{* *}$ & Silica-Stearyl & 116 & - & Sed,VD \\
DB360 $^{* *}$ & Silica-Stearyl & 110 & - & \\
\hline
\end{tabular}

*Commercial product obtained from microParticles GmbH, Germany. ${ }^{* *}$ Particles obtained from "Deeltjesbank" at Van 't Hoff Laboratory.

or water/ethanol mixtures. For each vertical deposition setup, particle type and size the experimental conditions, including particle concentration, solvent, evaporation temperature and substrate inclination, were optimized. A typical vertical deposition experiment consisted of the preparation of a series of $0.1,0.2,0.5,1$ and $2 \mathrm{v} \%$ polystyrene dispersions in water by diluting a desired amount of the stock dispersion with water to a total volume of $5 \mathrm{~mL}$. The dispersion was placed into a scintillation vial of $20 \mathrm{~mL}$ and a glass substrates were placed into the dispersions at a desired angle. The solvent was evaporated by drying in an oven at $55^{\circ} \mathrm{C}$ for $\sim 48 \mathrm{~h}$. The time needed for full evaporation of the $5 \mathrm{~mL}$ depends mostly on the solvent choice.

Glass substrates of various sizes were cut from glass slides of $24 \times 50 \mathrm{~mm}$ (Menzel Gläser \#1) to their desired size. The glass substrates were cleaned by soaking in a solution of $7.5 \mathrm{wt} \%$ potassium hydroxide (Merck, p.a.) in a mixture of ethanol (Merchachem, 100\%) and water (from a Millipore System) for the duration of $1 \mathrm{~h}$ although typically $16 \mathrm{~h}$ were employed.

Sedimentary colloidal crystals were only prepared of the silica particles because these can be dispersed in low density and volatile solvents, such as cyclohexane, ethanol and toluene. Non-crosslinked polystyrene particles will dissolve in most apolar solvents and their density difference with water is very low, $\Delta \rho=0.05 \mathrm{~g} / \mathrm{cm}^{3}$, which makes their sedimentation a very slow process. The high density difference between the Stöber silica and the solvents, $\Delta \rho \approx 1 \mathrm{~g} / \mathrm{cm}^{3}$, is favorable for sedimentation and in addition the solvents can be slowly evaporated at room temperature. The sedimentary colloidal crystal grains were obtained by drying the colloidal silica spheres dispersion in small flasks. Due to the volatile nature of the solvents, they slowly evaporated from the flasks over the course of several months or years. During this time the 
particle concentration slowly increased and crystals were formed, followed by full drying of the sediments. Crystalline sediments could be identified by the presence of strong Bragg reflections.

\subsubsection{Fiber preparation}

Carbon fibers of $10 \mu \mathrm{m}$ thick were provided by J. Gulden from Hasylab at DESY in Hamburg, Germany. The fibers were cut to a length of $5 \mathrm{~mm}$ using a razor blade and connected to glass

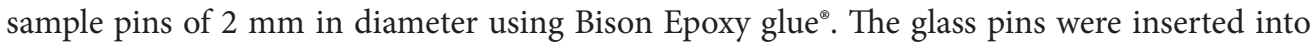
a magnetic adjustable crystal mount (Hampton Research) that can be placed on a goniometer head.

Colloidal crystal grains of various sizes were obtained by manual crushing a piece of the ordered sediments or by scraping a convective colloidal crystal film with a razor blade. Small individual grains of approximately $5 \mu \mathrm{m}$ were identified using a Leica M205 C Stereomicroscope having the advantage of a large working distance $(\sim 5 \mathrm{~cm})$ at high $(800 \times)$ magnification. The selected grains were picked up manually using a micromanipulator (PatchMan NP2, Eppendorf) and connected to the tip of a carbon fiber. The connection to the fiber was either caused by attractive electrostatic forces or by dipping the fiber into a very small amount of one of the components of Bison Epoxy glue $e^{\varpi}$ with the micromanipulator, this results in a sticky fiber surface but prevents the formation of glue drops that complicates crystal grain pick-up.

\subsubsection{Characterization of colloidal crystals}

The structure and thickness of the colloidal crystal films and grains were studied with scanning electron microscopy (SEM) with a Phenom G2 desktop SEM and Philips XLFEG30. SEM samples were prepared by breaking the substrate into smaller pieces (if necessary) that were connected to SEM stubs with conductive carbon tape, scratched with a razor blade to reveal their inner structure and sputter-coated with $6 \mathrm{~nm}$ platinum. Fibers with colloidal crystal grains were either connected to the SEM stubs via their glass pins with carbon tape or removed from the glass pins and placed onto the stub directly. Focused ion beam (FIB) lithography was performed with a FEI Nova Nanolab 600 Dualbeam apparatus, which uses Gallium ions to cut through structures.

\subsection{Results}

\subsection{Colloidal crystal films prepared via vertical deposition}

Colloidal crystal films of different particle composition, size and layer thickness were needed for different X-ray diffraction studies. Therefore, many different colloidal crystal films were prepared with the vertical deposition method. For the repeated fabrication of a large number of colloidal crystal films readily available materials were used that consisted of thin glass microscope slides as substrates and scintillation vials of $20 \mathrm{~mL}$ as dispersion containers. Other experimental control parameters, such as substrate angle, dispersion solvent and oven temperature were also optimized. Slides of $40 \times 12.5 \mathrm{~mm}$ in size were used because these fit into the scintillation vials easily and provide a substrate angle of $\sim 30^{\circ}$. Due to the angle, a colloidal 

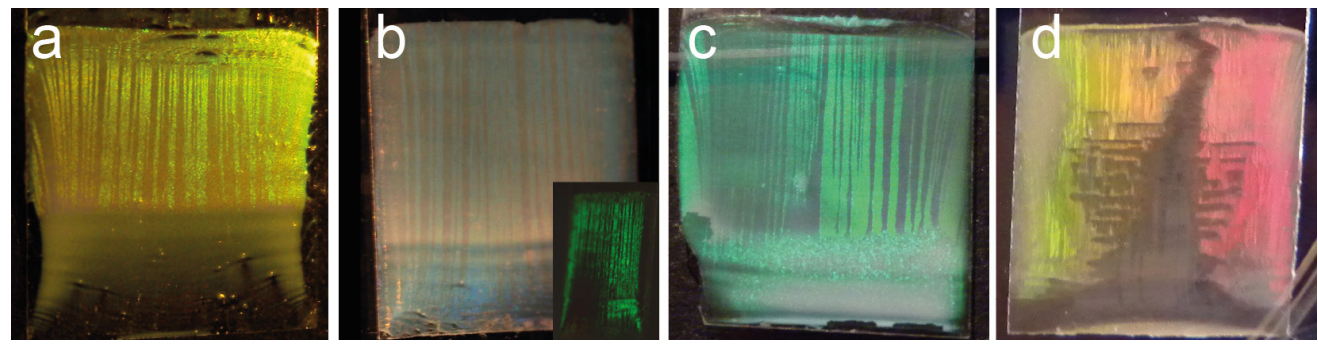

Figure 2.I. Photographs of colloidal crystal films of $10 \times 10 \mathrm{~mm}$ prepared from a $5 \mathrm{~mL}$ suspension of a) $0.25 \mathrm{v} \%$ BvR_I3 particles in water with $R=136 \mathrm{~nm}$ dried at $50{ }^{\circ} \mathrm{C}$. b) $0.25 \mathrm{v} \% \mathrm{PSI}$ particles in water with $R=208 \mathrm{~nm}$ dried at $55^{\circ} \mathrm{C}$, inset shows the film with a different orientation resulting in strong green Bragg reflections. c) $1.0 v \%$ DB275 particles in ethanol with $R=118 \mathrm{~nm}$ dried at $60{ }^{\circ} \mathrm{C}$ and d) $0.5 \mathrm{v} \%$ DB360 particles in ethanol with $R=246 \mathrm{~nm}$ dried at $70{ }^{\circ} \mathrm{C}$.

crystal film is only formed on the front side of the substrate. The best solvents were found to be water for polystyrene particles and ethanol for silica particles that should be evaporated around $50-55^{\circ} \mathrm{C}$ and $65-70^{\circ} \mathrm{C}$, respectively.

By using the optimal experimental conditions during the vertical deposition it was possible to produce colloidal crystal films that fully covered the substrate and that showed the presence of large single crystal regions. Figure 2.1.a shows photographs of typical colloidal crystal films of differently sized polystyrene and silica spheres prepared. Under white illumination these films show distinct Bragg reflections with different colors depending on particle size indicating the presence of ordered structures. The vertical stripes are the result of twinning crystal structures. Homogeneous full substrate covering films were obtained for all differently sized polystyrene particles and for silica particles with $R<150 \mathrm{~nm}$. Figures 2.1.a-c show three typical films obtained for BvR_I3, PS1 and DB275. However, for larger silica particle sizes, the films were usually found to be inhomogeneous. Due to the relatively fast sedimentation of the particles, their flux towards the meniscus decreases over time. For TPM coated silica particles DB360 with $R=246 \mathrm{~nm}$ a typical film is shown in Figure 2.1.d, where a large uncovered part is present in the middle part and the thickness of the film clearly decreases towards the bottom of the substrate. For all colloidal crystal films the thickness was found to become less homogeneous towards the bottom part of the substrate. This is caused by a change in meniscus shape at this solvent height induced by the close proximity of the bottom and walls of the scintillation flasks changing the solvent wetting angle and thus the particle influx.

SEM measurements showed that the colloidal spheres crystalized into a face centered cubic (FCC) structures, with the (111) face parallel to the glass substrate as expected ${ }^{[13]}$. Figure 2.2 shows the SEM images of colloidal crystal films of polystyrene particles PS1. The films contains large cracks that run from top to bottom (Fig.2.2.a), this is a commonly observed drying effect caused by tensile stresses during solvent evaporation decreasing the distance between the particles. In the SEM images additional shrinkage is caused by the exposure to electrons and the high vacuum in the $\mathrm{SEM}^{[14]}$. Depending on the number of layers the distance between the 

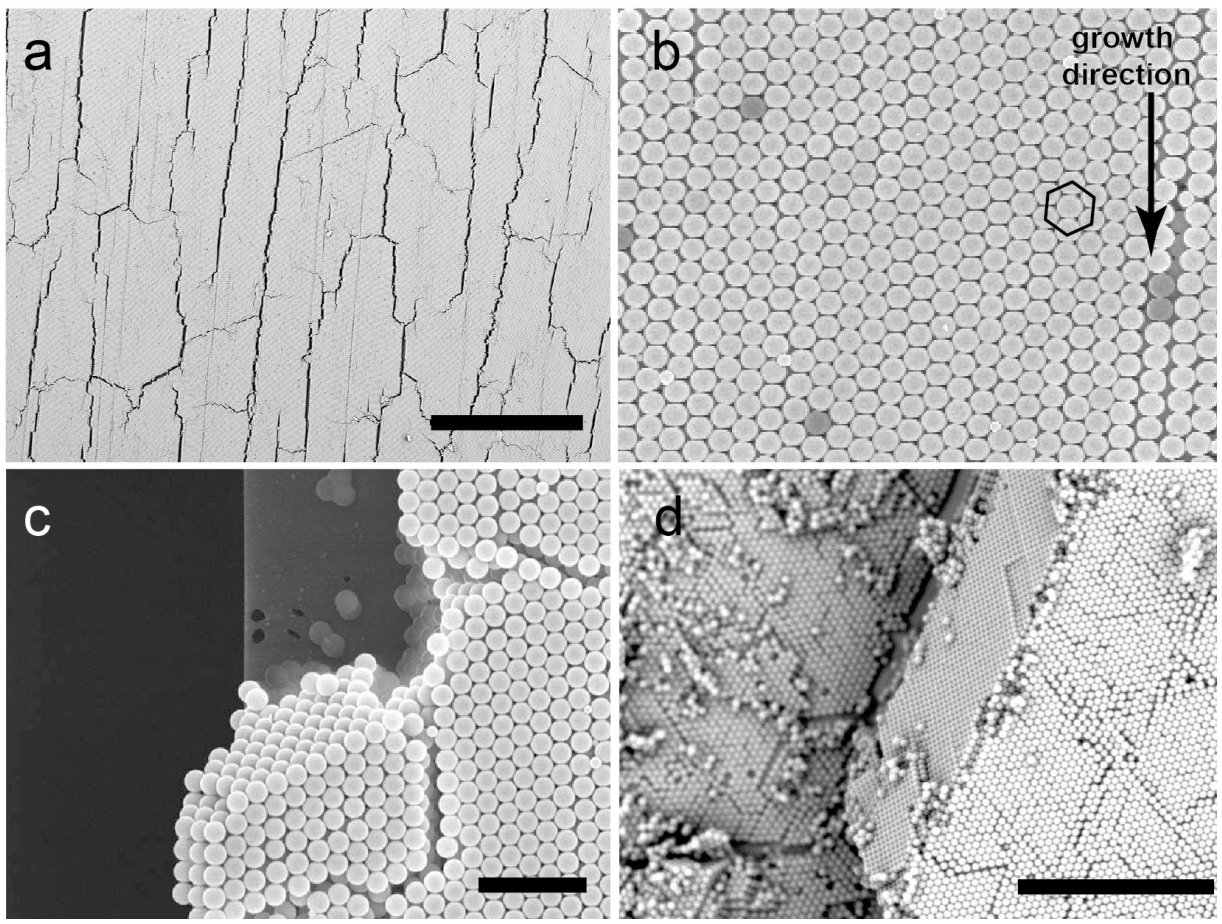

Figure 2.2. SEM images of a colloidal crystal film of polystyrene particles PSI. a) Overview of large area that shows drying cracks. b) A higher magnification of the colloidal crystal film top layer where the particles are ordered in a hexagonal arrangement. c) The edge of a crystal consisting of 9 layers and d) a scratched region of a crystal with $>30$ layers. Scale bars are a) $50 \mu \mathrm{m}$, b,c) $2 \mu \mathrm{m}$ and d) $10 \mu \mathrm{m}$.

cracks changes. For films of polystyrene particles consisting of only a few layers the cracks are much smaller. Films prepared from silica particles showed less cracks, because these particles shrink less in size than the polystyrene particles upon solvent evaporation. Furthermore, it has been shown for silica particles that sintering at high temperatures can prevent the crack formation.

Figure 2.2.b shows the top layer of the crystal, where the hexagonal arrangement of the spheres in the layers can be clearly seen as indicated by the hexagon. The orientation of the hexagonal order in the layers was consistently found to be aligned within $\sim 15^{\circ}$ along the growth direction as indicated by the arrow. Why this orientation is obtained is not clear but could be related to the growth process that involves several transitions in layer thickness at the start and is furthermore influenced by the solvent flux. For more details on the growth process see refs [15-17].

The SEM measurements also provide insight into the layer thickness of the crystals. By scraping the crystal with a razor blade the inner structure of the crystal and the number of layers could be estimated. As expected the thickness of the films was controlled by the particle 
concentration if all other conditions were kept constant. Figure 2.2.c shows a crystal of polystyrene spheres consisting of 9 layers while Figure 2.2.d shows a crystal where at least 30 layers can be distinguished. The number of layers was found to increase rapidly at the start of the film followed by a region with an almost constant number of layers. The films increased only with a few layers over a range of a few $\mathrm{mm}$. At the end of the films the thickness was usually found to increase rapidly and the structure became disordered. This is caused by a higher particle flux as a result of the increase of the particle concentration caused by the solvent evaporation and the change in meniscus shape, as discussed previously.

The growth process was also performed on different substrates, such as silicon wafers coated with silicon nitride $\left(\mathrm{Si}_{3} \mathrm{~N}_{4}\right)$ membranes of different thickness and size. Because the wetting of $\mathrm{Si}_{3} \mathrm{~N}_{4}$ is very different from that of $\mathrm{SiO}_{2}$ the $\mathrm{VD}$ conditions had to be optimized for these studies. For Si wafers of $23 \times 23 \mathrm{~mm}$ with an array of windows sized $250 \times 250 \mu \mathrm{m}$ the optimal conditions were a $0.1 \mathrm{v} \%$ dispersion of polystyrene spheres in ethanol placed into scintillation vial of which the screw top was removed and dried at $25^{\circ} \mathrm{C}$ over several days.

\subsubsection{Single colloidal crystals grains on a fiber tip}

For CXDI measurements one of the requirements is that the colloidal crystal grains should be much smaller than the coherence length of the $\mathrm{x}$-ray beam to ensure coherent irradiation. For the experiment described below the goal was to have a grain size around $5 \mu \mathrm{m}$. Colloidal crystals obtained via sedimentation or vertical deposition were used as source for the grains.

The sedimentary crystals were prepared by slow settling of silica particle dispersions. Figure 2.3. a shows the crystallization of the TPM coated silica particles DB239 upon sedimentation in ethanol/toluene mixture. Clear Bragg reflections can be seen from the top of the sediment indicating the presence of crystals. After drying the obtained colloidal crystal sediments were manually broken into smaller pieces and the crystal quality was checked. Figure 2.3.b shows the surface of a small crystal grain consisting of DB284 particles imaged with light microscopy that shows the presence of different orientated grains, indicated by the Bragg reflection of different
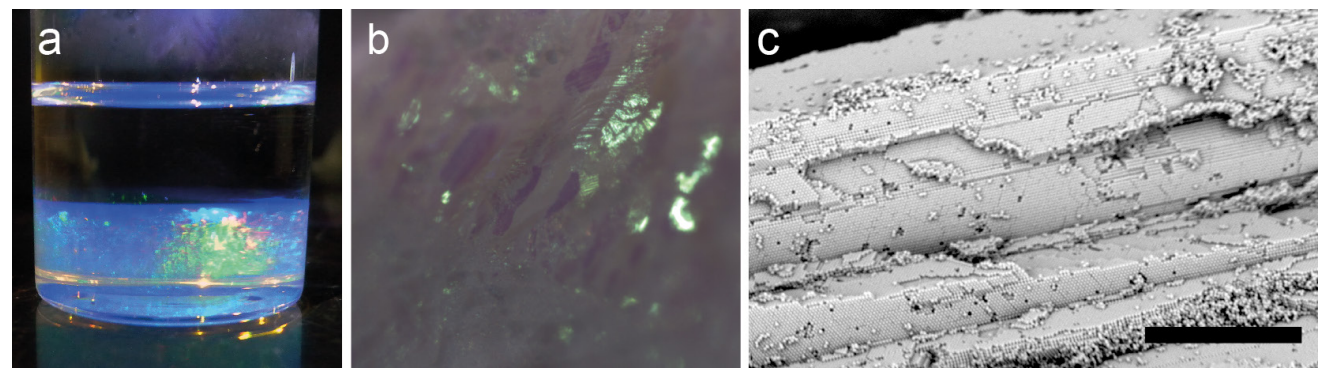

Figure 2.3. a) Photograph of the sedimentation of TPM coated silica spheres DB284 in an ethanol toluene mixture. b) Light Microscopy image of the surface of a large colloidal crystal grain of stearyl coated silica spheres obtained after drying and breaking of an ordered sediment and c) SEM image of the edge of a colloidal crystal grain of silica spheres. Scale bar is $10 \mu \mathrm{m}$. 

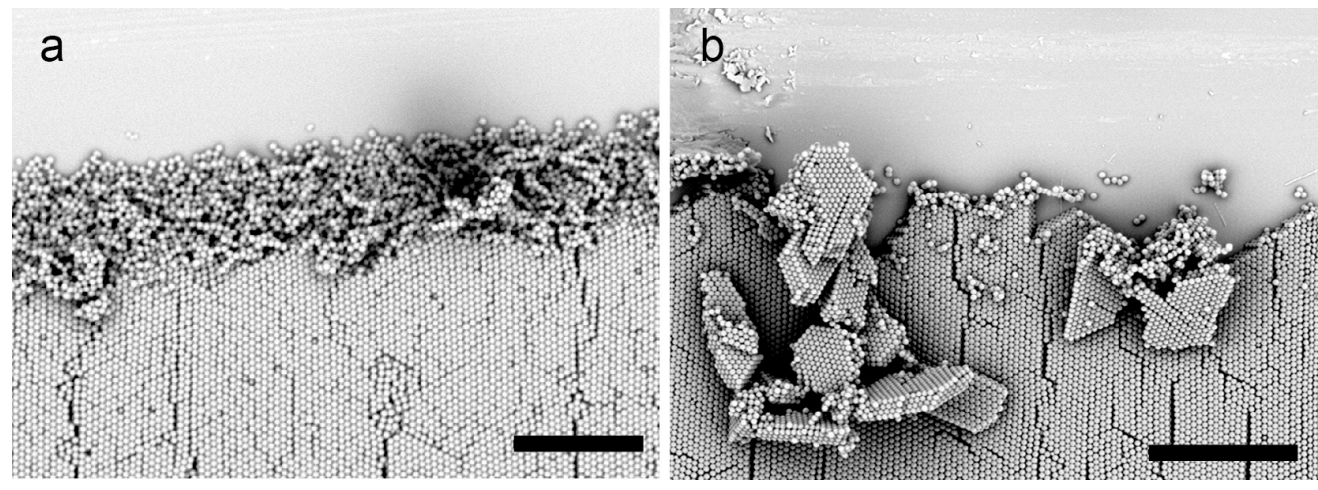

Figure 2.4. SEM image of colloidal crystal film close to a scratch of a) silica particles DB360 coated with TPM and b) polystyrene particles PSI. Scale bars are $10 \mu \mathrm{m}$.

colors, as expected for a sedimentary crystal. SEM measurements revealed the presence of a large number of ordered layers in the colloidal crystal grains. Figure 2.3.c. shows the surface of a large grain consisting of DB359 particles where the layers can be clearly distinguished. The rough edges, particle clusters and vacancies indicate that the mechanical crushing does damage the crystals slightly but the overall order is retained, making them suitable candidates for the preparation of small colloidal crystal grains.

With the vertical deposition method colloidal crystal films were prepared from silica and polystyrene particles as described in Section 2.3. To obtain colloidal crystal grains the films were scratched with a razor blade. Figure 2.4 shows the effect of scratching on the crystals made from polystyrene spheres and TPM coated silica spheres. The crystal of silica spheres is destroyed close to the scratch and a random structure of spheres is left behind. The crystal of polystyrene spheres shows the presence of small chunks of crystals that have come lose off the glass substrate. This difference in behavior can be explained by a difference in interaction between the particles and between the particles and the substrate. The silica particles are in contact through their TPM coating layers that apparently deform easily. While the polystyrene particles seem to have formed strong bonds and the crystals break along the drying cracks which preserves the crystal order. Therefore, the colloidal crystal films of polystyrene spheres were used as source for their grains. With the additional advantage that the crystal grain thickness can be controlled with the initial particle concentration in the VD experiment.

The next step was selecting the grains with a proper size of $\sim 5 \mu \mathrm{m}$ and connecting these to the tip of a carbon fiber. The grains were obtained by manually smashing macroscopically large colloidal crystal grains. As this is not a controlled process and the larger grains are likely to break at the points of least resistance, a collection of grain sizes is obtained. Figure 2.5.a shows a microscopy image obtained at the highest magnification $(800 \times)$ of the stereo microscope where the arrows indicate a few grains of suitable size. The inset shows the full overview of all different grain sizes that are present. It is impossible to determine in this step whether the grains have retained their crystalline order. However, the crystal quality of the obtained crystal grains 


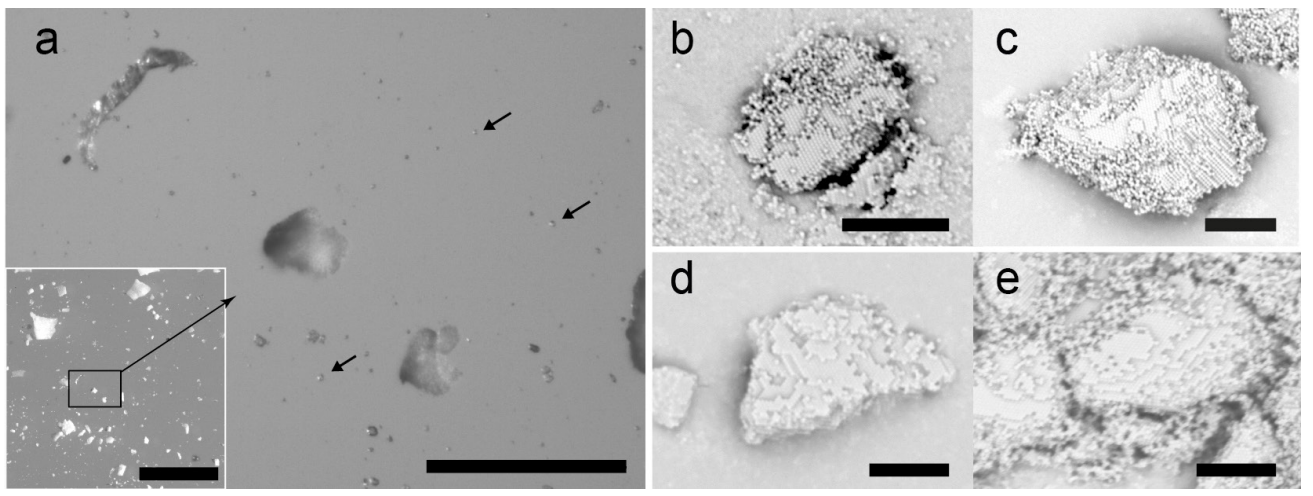

Figure 2.5. a) Stereo microscopy image at highest magnification of variously sized colloidal crystal grains of particles DB359, grains of $\sim 5 \mu \mathrm{m}$ are indicated with the arrows (inset) low magnification image showing the large deviation in grain sizes obtained after crushing. b-d) SEM image of a small colloidal crystal grains consisting of; b) DB359 c) DB284 d-e) DB275. In most grains the ordered layers can be observed indicating the order is preserved during crushing for these silica particles. Scale bars are a) 200 $\mu \mathrm{m}$ (inset I mm), b-e) $5 \mu \mathrm{m}$.

from the bulk sediments was checked with the SEM. Figure 2.5.b-e shows a few examples of crystalline grains obtained from several different silica particles. The grains show the presence of a number of differently sized layers. Besides these crystalline grains it is clear that also amorphous structures are present. One can therefore never be sure if the picked up grain is truly a colloidal crystal grain.

After selection of a properly sized grain, it was connected to a carbon fiber by bringing the fiber in close contact with the grain by use of a micromanipulator. The connection was made via either electrostatic forces or using (a single component of) epoxy glue. The first will not produce background during the CXDI measurement but has a low success rate, while the latter will increase the background and might even destroy the grain but provides a very stable bond.

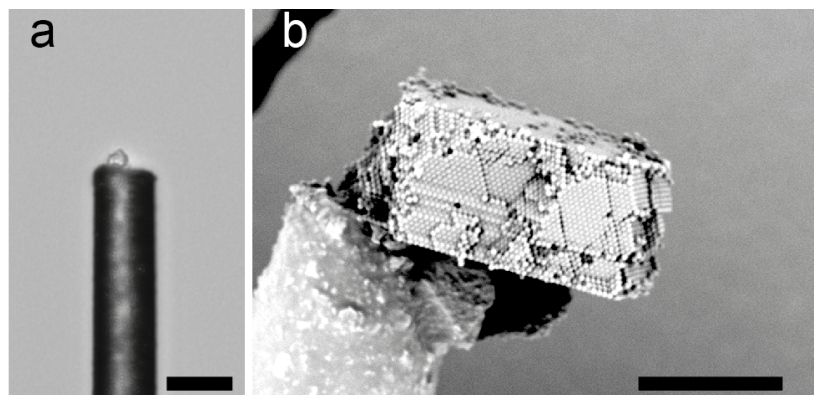

Figure 2.6. a) Microscopy image of a colloidal crystal grain on a carbon fiber tip. SEM image of b) a colloidal crystal grain of polystyrene particles attached by glue to the fiber tip after pickup. Scale bars are a,b) $10 \mu \mathrm{m}$. 
Figure 2.6.a shows an example of a colloidal crystal grain on a fiber tip after successful pickup. A SEM image of a polystyrene sphere crystal grain picked up with glue is shown in Figure 2.6.b. For the electrostatic connected grains SEM imaging is impossible as the additional charging by the electrons destroys the bond between grain and fiber. The SEM image shows that the crystalline order is still present and several different layers can be distinguished. The SEM analysis also showed that the selection does not always result in grains of proper size.

CXDI experiments were performed with a large number of the picked up colloidal crystal grains. These measurements showed that many conditions have to be right for a successful CXDI experiment. It turned out that from all prepared crystals on a fiber ( $>200$ samples) only two or three crystal grains produced scattering data of proper quality. There were several reasons for this low success rate. First of all, the measurements showed that the colloidal crystals were easily removed from the fibers. Secondly, not all of the selected grains were found to be crystalline. Thirdly, silica particles produced much better signals than polystyrene particles of the same size. The higher electron density of silica provides a much better X-ray contrast and hence signal-to-noise ratio than the polystyrene spheres. Although SEM measurements showed the polystyrene crystals always retain their crystal order upon breaking, even very large crystal grains $(>20 \mu \mathrm{m})$ did not provide a proper signal. However, when a properly connected colloidal crystal grain consisting of silica particles that retained its crystal structure was imaged, high quality scattering data could be obtained over a full $180^{\circ}$ rotation.

\subsection{Conclusions \& Outlook}

The vertical deposition method was applied succesfully for the preparation of colloidal crystal films of polystyrene and silica particles. By tuning the experimental vertical deposition parameters, the crystal growth process was controlled and the desired thickness and surface covering on different substrates was achieved. The obtained crystals display a similar crystal orientation and thickness over a large sample area and makes them very suitable for X-ray diffraction studies.

Two different kinds of synchrotron X-ray diffraction studies were performed of the colloidal crystal films. A pump-probe study of the effect of strong optical fields on the colloidal crystal and the particle dynamics was performed and is described in section 2.5.1 Also a microradian $\mathrm{X}$-ray diffraction ( $\mu \mathrm{rad}-\mathrm{XRD}$ ) study was performed to clarify the effect of temperature on the structure and bulk properties of the colloidal crystals and is described in section 2.5.2.

For CXDI measurements colloidal crystal grains are needed and it was shown that the most suitable particles are coated silica spheres. The colloidal crystals of the silica spheres should be prepared via slow sedimentation followed by drying because the grains produced from these sediments retain their crystalline order upon sediment fracture, in contrast to convectiveassembly crystals. In addition, the high X-ray contrast of the silica resulted in the strongest CDXI signals.

In the future, the process of crystal grain selection could be improved. Now the grain selection results in grains of improper size and/or non-crystallinity. A way to improve the 

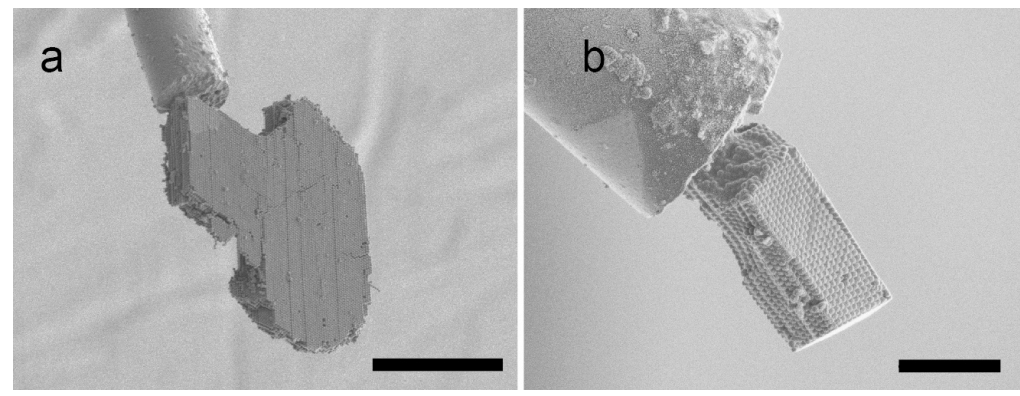

Figure 2.7. SEM images of the FIB cutting process of sizing down a) a large colloidal crystal grain of polystyrene spheres to b) a size of $5 \times 10 \mu \mathrm{m}$. Scale bars are a) $20 \mu \mathrm{m}$ and b) $5 \mu \mathrm{m}$.

selection is employing Focused Ion Beam (FIB) processing. With this method colloidal crystal grains could be cut to any desired size. Preliminary experiments showed that this can indeed be achieved. Figure 2.7 shows the sizing down procedure of a large crystal grain of polystyrene spheres (Fig.2.7.a) milled down to a smaller size (Fig. 2.7.b). Clearly, the FIB technique is a very suitable technique for the preparation of properly sized crystal grains. In addition, the in-situ SEM imaging provides direct information about the crystal quality and makes the search for proper colloidal crystal grains easier.

\subsection{X-ray diffraction experiments of colloidal crystals of spheres \\ 2.5. I. Pump-probe experiments with free electron lasers}

The colloidal crystal films of polystyrene spheres described in section 2.3 were used for several studies. In this section a short description is given of the experiment and results of pump-probe experiments of these films as reported by Dronyak et al. ${ }^{[18]}$.

The self-assembled colloidal crystal films of polystyrene films are of interest for the bottomup fabrication ${ }^{[19]}$ of a new class of nano-materials, such as hypersonic phononic crystals ${ }^{[20]}$. It has been shown that colloidal crystals of polystyrene spheres can possess a phononic band gap in the gigahertz frequency range ${ }^{[21]}$. To characterize and understand these properties the ultrafast dynamics in the colloidal crystal were studied with an advanced free-electron laser (FEL). Time-resolved experiments of the temporal changes in the sample induced by infrared (IR) laser pulses of $800 \mathrm{~nm}$ and imaged with soft X-rays with a wavelength of $8 \mathrm{~nm}(154.98 \mathrm{eV})$ at the BL3 beamline were performed at FLASH. Figure 2.8 shows a schematic representation of the performed experiment. A single IR pulses was used to pump the sample, followed by a single FEL pulse with a time delay, $\Delta t$, to probe the instantaneous structure. The time delay could be controlled with picosecond resolution and time delays of -100 to +1000 ps were used. The sample was a colloidal crystal film consisting of polystyrene particles on a $\mathrm{Si}_{3} \mathrm{~N}_{4}$ membrane of $100 \mathrm{~nm}$ thick. The diffraction patterns produced by the transmitted FEL beam were collected by a detector at $17.4 \mathrm{~cm}$ behind the sample.

Analysis of the Bragg peak position, intensity and shape was performed to reveal the effect of 

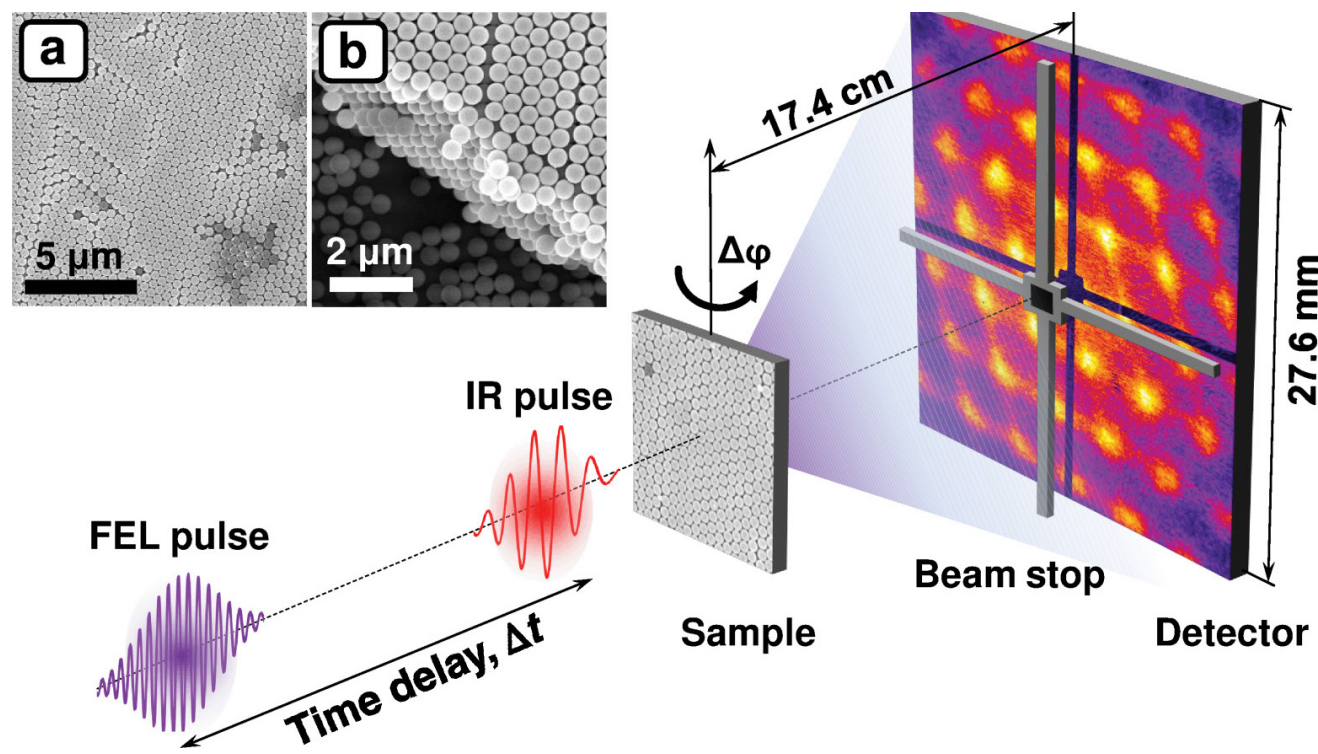

Sample

Detector

Figure 2.8. Schematic view of the pump-probe experiment showing the IR pump (IR pulse) and the soft $\mathrm{X}$-ray probe (FEL pulse) separated by a time delay $\Delta t$, the sample, and the detector, which is protected by the beam stop. Insets (a) and (b) show SEM images of the colloidal crystal film used in the experiment. The II layers of polystyrene spheres composing the colloidal crystal are visible in the inset (b). Reproduced with kind permission from ref. [18]. Copyright 2013 American Physical Society.

the IR pulse on the crystal and the colloidal spheres. Fourier transforms of the time dependent parameters was used to determine the characteristic frequencies. This showed that an increased contribution was present in the frequency region of 4-5 GHz. Comparing this result to the Lamb theory showed that the breathing mode of the polystyrene spheres was probed in the

(I)

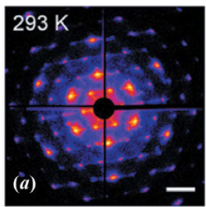

(II)

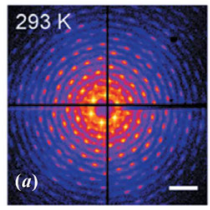

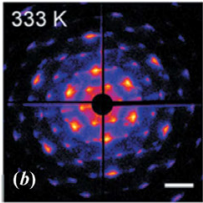

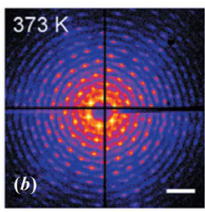

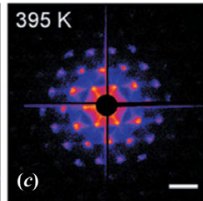

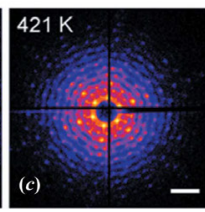

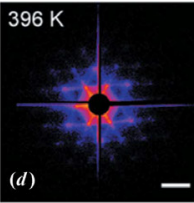

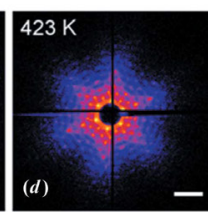

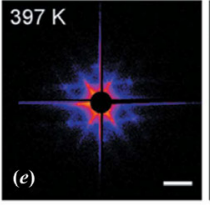
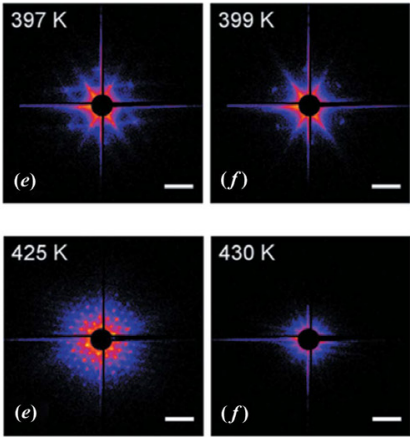

Figure 2.9. In situ diffraction patterns of colloidal crystals consisting of polystyrene spheres PS2 with $R=82 \mathrm{~nm}$ (top row) and PSI with $R=208 \mathrm{~nm}$ (bottom row) measured during incremental heating. The sample temperature is marked at the top left corner for each of the displayed patterns. Reproduced with permission of the International Union of Crystallography from ref. [22]. 
experiments.

A follow-up experiment was performed in April 2014 at the Linac Coherent Light Source (LCLS), at SLAC in Stanford, USA, together with the Hamburg team headed by Prof. Ivan Vartanyants. The results are currently being analyzed.

\subsubsection{In situ structural evolution upon heating with X-ray diffraction}

Another study that was performed of the colloidal crystal films of polystyrene films was focused on the structural evolution of the crystal upon heating as reported by Zozulya et al. ${ }^{[22]}$. This study focused on the changes in the colloidal crystals upon heating, as it has been shown that with elevated temperatures the photonic properties of a colloidal crystal film can be modified ${ }^{[23-25]}$. Furthermore, the particle size, which increases the surface-to-volume ratio, will affect the glass transition temperature of polystyrene. ${ }^{[26-28]}$ Understanding both effects is important for the potential use of the polystyrene crystals as photonic materials. For the in situ investigation of the effect of temperature, X-ray diffraction is very well suited due to the high penetration depth and nondestructive characterization approach.

The experiment was performed at the P10 coherence beamline of the PETRAIII synchrotron facility at DESY, Hamburg. Colloidal crystal films prepared on glass microscope slides from two differently sized linear polystyrene spheres, PS2 $R=82 \mathrm{~nm}$ and PS1 $R=208 \mathrm{~nm}$, were used. The films were slowly heated with small temperature steps over a range of $293 \mathrm{~K}$ tot $430 \mathrm{~K}$. Diffraction patterns were measured with a photon energy of $15 \mathrm{keV}$ and were collected by a detector located $5.2 \mathrm{~m}$ behind the sample.

Figure 2.9 shows the diffraction patterns of the colloidal crystal films consisting of the two differently sized polystyrene particles. Upon a temperature rise the deterioration and disappearance of the higher order Bragg peaks was observed, followed by enhancement of the diffuse scattering with hexagonal symmetry. This indicated that the polystyrene particles first slowly deformed into a dodecahedron shape before completely melting. The crystal lattice was also found to shrink slightly upon heating and finally completely disappeared.

From the total scattered intensity the glass transition temperature was determined to be $30 \mathrm{~K}$ lower for the smaller particle PS2 than the larger particles PS1. This showed that the glass transition temperature of the polystyrene is strongly affected by the particle size and hence the surface-to-volume ratio. 


\section{Acknowledgements}

This work is the result of the many collaborations with the group of Ivan Vartanyants from Hasylab and Alexey Zozulya and Michael Sprung of the P10 beamline staff of PETRAIII, both located at DESY in Hamburg, Germany. Johannes Gulden, Anatoly Shabalin, Roman Dronyak, Ruslan Kurta, Oleksandr Yefanov, Dmitry Dzighaev, Oleg Gorobstov, Andrej Singer and Ulf Lorenz are thanked for their help during the X-ray measurements. Jan Hilhorst is acknowledged for his help with the initial vertical deposition experiments. Bas van Ravensteijn and Daniela Kraft are thanked for kindly providing two of the polystyrene particles. Matthijs de Winter is thanked for performing the FIB experiments.

\section{References}

1. J. F. Galisteo-Lopez, M. Ibisate, R. Sapienza, L. S. Froufe-Perez, A. Blanco and C. Lopez, Adv. Mater, 2011,23, 30-69.

2. A. C. Blanco E., S. Grabtchak, M. Ibisate, S. John, S. Leonard, C. Lopez, F. Meseguer, H. Miguez, J. Mondia, G. Ozin, O. Toader and H. M. van Driel, Nature, 2000, 405, 437-40.

3. Y. Vlasov, X. Z. Bo, J. C. Sturm and D. J. Norris, Nature, 2001, 414, 289-93.

4. A. Bosak, I. Snigireva, K. S. Napolskii and A. Snigirev, Adv Mater, 2010, 22, 3256-3259.

5. J. Hilhorst, M. M. van Schooneveld, J. Wang, E. de Smit, T. Tyliszczak, J. Raabe, A. P. Hitchcock, M. Obst, F. M. F. de Groot and A. V. Petukhov, Langmuir, 2012, 28, 3614-3620.

6. D. V. Byelov, J. M. Meijer, I. Snigireva, A. Snigirev, L. Rossi, E. van den Pol, A. Kuijk, A. Philipse, A. Imhof, A. van Blaaderen, G. J. Vroege and A. V. Petukhov, RCS Advances, 2013, 3, 15670-15677.

7. J. Gulden, O. M. Yefanov, A. P. Mancuso, V. V. Abramova, J. Hilhorst, D. Byelov, I. Snigireva, A. Snigirev, A. V. Petukhov and I. A. Vartanyants, Phys. Rev. B, 2010, 81, 224105.

8. J. Gulden, O. M. Yefanov, A. P. Mancuso, R. Dronyak, A. Singer, V. Bernatova, A. Burkhardt, O. Polozhentsev, A. Soldatov, M. Sprung and I. A. Vartanyants, Opt. Express, 2012, 20, 4039-4049.

9. J. R. Fienup, Appl. Opt., 1982, 21, 2758-2769.

10. V. Elser, JOSA A, 2003, 20, 40-55.

11. K. A. Nugent, Adv. Phys., 2010, 59, 1-99.

12. a) A.P. Philipse, A. Vrij, J. Colloid Interface Sci., 1989,128, 121-126 b) A. K. Van Helden, J. W. Jansen and A. Vrij, J. Colloid Interface Sci., 1981, 81, 354-368.

13. P. Jiang, J. F. Bertone, K. S. Hwang and V. L. Colvin, Chem. Mater., 1999, 11, 2132-2140.

14. N. Denkov, O. Velev, P. Kralchevski, I. Ivanov, H. Yoshimura and K. Nagayama, Langmuir, 1992, 8, 3183-3190.

15. L. Meng, H. Wei, A. Nagel, B. J. Wiley, L. E. Scriven and D. J. Norris, Nano Lett., 2006, 6, 2249-53.

16. D. D. Brewer, J. Allen, M. R. Miller, J. M. D. Santos, S. Kumar, D. J. Norris, M. Tsapatsis and L. E. Scriven, Langmuir, 2008.

17. D. J. Norris, E. G. Arlinghaus, L. Meng, R. Heiny and L. E. Scriven, Adv. Mater, 2004, 16, 1393-1399.

18. R. Dronyak, J. Gulden, O. M. Yefanov, A. Singer, T. Gorniak, T. Senkbeil, J. M. Meijer, et al. Phys. Rev. B, 2012, 86, 064303.

19. F. Marlow, P. Sharifi, R. Brinkmann and C. Mendive, Angew. Chem., 2009, 48, 6212-33.

20. T. Gorishnyy, C. Ullal, M. Maldovan, G. Fytas and E. Thomas, Phys. Rev. Lett., 2005, 94, 115501.

21. W. Cheng, J. Wang, U. Jonas, G. Fytas and N. Stefanou, Nature Mater., 2006, 5, 830-836.

22. A. V. Zozulya, J. M. Meijer, A. Shabalin, A. Ricci, F. Westermeier, R. P. Kurta, U. Lorenz, A. Singer, O. Yefanov, A. V. Petukhov, M. Sprung and I. A. Vartanyants, J. Appl. Cryst., 2013, 46, 903-907.

23. H. Miguez, F. Meseguer, C. Lopez, A. Blanco, J. S. Moya, J. Requena, A. Mifsud and V. Fornes, Adv. Mater., 1998, 10, 480-483.

24. B. Gates, S. H. Park and Y. N. Xia, Adv. Mater., 2000, 12, 653-656.

25. T. Deng, J. Zhang, K. Zhu, Q. Zhang and J. Wu, Mater. Chem. Phys., 2011, 129, 540-546.

26. J. L. Keddie, R. A. L. Jones and R. A. Cory, Europhys. Lett., 1994, 27, 59-64.

27. J. A. Forrest, K. Dalnoki Veress, J. R. Stevens and J. R. Dutcher, Phys. Rev. Lett., 1996, 77, 2002-2005.

28. O. Baeumchen, J. D. McGraw, J. A. Forrest and K. Dalnoki-Veress, Phys. Rev. Lett., 2012, 109, 055701. 


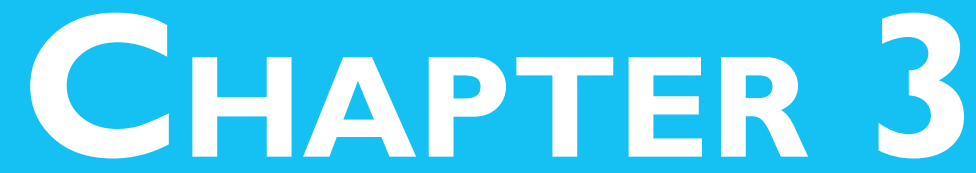

-

Double Hexagonal Close Packed Structure ReVEaled IN a Single Colloidal CRYSTAL GRAin by BRAgG ROD ANALYSIS

Abstract

In this chapter we report on the coherent X-ray diffraction imaging study of a single colloidal crystal grain composed of silica spheres. The diffraction data contained Bragg peaks and Bragg rods, which are related to the stacking of the hexagonally close packed layers. The profile of the Bragg rod showed distinct intensity modulations that under our specific experimental conditions are directly related to the stacking sequence of the layers. Using a model for the scattered intensity along a Bragg rod of an exact stacking of a finite number of hexagonally close packed layers, we show that a double hexagonal close packed stacking sequence is present in the colloidal crystal grain. This analysis method opens up ways to obtain crucial structural information from finite sized crystalline samples by employing advanced third generation X-ray sources. 


\section{I. Introduction}

Crystal defects have been extensively studied with X-ray diffraction for decades ${ }^{[1]}$. The displacement of crystal planes in the lateral directions, known as stacking disorder, results in the appearance of diffuse scattering along lines, so-called Bragg rods, between the Bragg peaks. As shown by the work of Wilson on cobalt structures, statistical analysis can relate the intensity of the Bragg rods, to the average defect structure ${ }^{[2]}$.

For colloidal crystals of spheres the equilibrium structure is predicted to be a face centered cubic (FCC) crystal. However, in stacking sequences of the hexagonal close-packed layers planar stacking faults are often observed resulting in a random hexagonal close-packed (RHCP) structure, as also discussed in Chapter 1 , Section $1.3^{[3]}$. This is related to the very low energy difference between the two crystal structures of less than $10^{-3} k T$ per particle ${ }^{[4-6]}$, where $k$ is the Boltzmann constant and $T$ the absolute temperature.

Due to the stacking disorder Bragg rods have also been observed in the diffraction data of colloidal crystals and have provided insight into the RHCP stacking sequences ${ }^{[7-10]}$. However, all investigations concerned statistically-averaged data of large $(>100 \mu \mathrm{m})$ sample areas. Therefore, the intensity along the Bragg rods is smeared and only provided information on the average defect density. Now, with the advancement of the X-ray scattering techniques and sources it has become possible to measure smaller crystal sizes with coherent X-ray diffraction imaging (CXDI) ${ }^{[11,12]}$. As a result the diffuse scattering data becomes more pronounced and can be directly related to the real space structure and stacking sequence.

In this chapter we study a small, about $4 \mu \mathrm{m}$, single colloidal crystal grain of silica spheres using CXDI. In the scattering patterns intense diffuse scattering between the Bragg peaks is observed including Bragg rods with distinct intensity modulations. The Bragg rod intensity profiles are directly related to the exact RHCP stacking sequence. By fitting a scattering model based on the stacking sequence of a finite number of hexagonally close-packed layers to the Bragg rod intensity profile, we can, in contrast to the earlier studies, now reveal the specific stacking sequence of layer positions without any assumptions of the stacking sequence beforehand. This analysis is the main focus of this chapter and will be explained in detail. Another way of analyzing the CXDI data is by applying iterative phase retrieval algorithms ${ }^{[13-15]}$. The CDXI method reconstructs the $3 \mathrm{D}$ electron density of the studied object from which the particle positions can be obtained. The $3 \mathrm{D}$ analysis provides direct insight into the real space structure of the colloidal crystal grain. Both approaches enabled us to access the structural information of this finite-sized object on the basis of coherent diffraction measurements and are in good agreement.

\subsection{Experimental}

The samples were prepared from dried sediments of colloidal crystals that showed characteristic optical Bragg reflections as described in Chapter 2, Section 2.3.2. The colloidal crystal grain used in this study consisted of silica spheres DB359 with radius $R=110 \mathrm{~nm}$ that was dried from cyclohexane over several months. The colloidal crystal grain used was imaged with light microscopy and determined to have volume of about $2 \times 3 \times 4 \mu \mathrm{m}^{3}$. 
The experiment was performed at the coherence beamline P10 of the PETRA III synchrotron at DESY, Hamburg, Germany ${ }^{[16]}$. A monochromatic X-ray beam of $8 \mathrm{keV}$ photon energy was focused at the sample at $87.7 \mathrm{~m}$ distance from the source using the transfocator optics ${ }^{[17]}$ based on compound refractive lenses (CRLs) ${ }^{[18-20]}$. The CRL optics were positioned at $2.2 \mathrm{~m}$ distance upstream from the sample, and a slit system of $75 \times 75 \mu \mathrm{m}$ in size located at $1.5 \mathrm{~m}$ distance before the CRL, was used to select a coherent portion of the beam. The size of the focal spot at the sample position was $5.5 \mu \mathrm{m}$ (FWHM) in horizontal and $3.2 \mu \mathrm{m}$ (FWHM) in the vertical directions with about $10^{11}$ photons per second of total intensity. To inhibit radiation damage the grain was cryocooled with a nitrogen flow. Coherent diffraction patterns were acquired using a MAXIPIX detector with $516 \times 516$ pixels and a pixel size of $55 \times 55 \mu \mathrm{m}$. The detector was located at $5.1 \mathrm{~m}$ distance from the sample with a minimum resolution in reciprocal space of $0.4372 \mu \mathrm{m}^{-1}$ defined by the geometry of the experiment and pixel size. An evacuated flight tube was used between the sample and the detector in order to reduce background scattering. A full dataset consisted of a rotation series of 360 diffraction patterns with $0.5^{\circ}$ increment, thus covering the entire reciprocal space. At each angular position a series of 100 diffraction patterns was measured to provide a good signal to noise ratio. To access the scattering signal close to the directly transmitted beam the semi-transparent beamstop made of $300 \mu \mathrm{m}$ thick Si wafer $\left(3 \times 3 \mathrm{~mm}^{2}\right.$ area $)$ was installed in front of the detector. Additionally, a Ta disk of $0.5 \mathrm{~mm}$ in diameter was glued on top of Si wafer to absorb the direct beam completely.

\subsection{Bragg rod analysis}

\subsection{Theoretical model}

In this work we analyze the diffraction data along a Bragg rod of a single colloidal crystal grain consisting of a finite number of close-packed hexagonal layers ${ }^{[2]}$. To be able to extract the exact RHCP stacking sequence of the layers we start by describing the real space structure of colloidal crystal by the hexagonal set of basis vectors $\mathbf{a}_{1}, \mathbf{a}_{2}$ and $\mathbf{a}_{3}$ as shown in Figure 3.1.a. The hexagonal layers are described by $\mathbf{a}_{1}$ and $\mathbf{a}_{2}$, with $\left|\mathbf{a}_{1}\right|=\left|\mathbf{a}_{2}\right|=a$, which is the interparticle distance, and the inter-layer spacing is described by the vector $\mathbf{a}_{3}$. The layers are stacked along the $\mathbf{a}_{3}$ or [001] direction and the order of the A, B or C layer positions, or respective lateral displacements, determines the stacking sequence. We define the lateral displacements as 'forward' for $\mathrm{A}$ to $\mathrm{B}$, $\mathrm{B}$ to $\mathrm{C}$ or $\mathrm{C}$ to $\mathrm{A}$ or 'backward' for $\mathrm{A}$ to $\mathrm{C}, \mathrm{B}$ to $\mathrm{A}$ or $\mathrm{C}$ to $\mathrm{B}$. This displacement can be described in-plane by the 'forward' vector $\mathbf{c}=\left(2 \mathbf{a}_{1} / 3\right)+\left(\mathbf{a}_{2} / 3\right)$ and the 'backward' vector $-\mathbf{c}$ (see Fig. 3.1.b).

Based on the hexagonal set of direct space basis vectors the reciprocal basis vectors $\mathbf{b}_{\mathbf{1}}$, $\mathbf{b}_{\mathbf{2}}$, and $\mathbf{b}_{3}$ can be determined in the usual way ${ }^{[1]}$. The scattering vector $\mathbf{q}$ is then described by $\mathbf{q}=h \mathbf{b}_{1}+k \mathbf{b}_{2}$ $+l \mathbf{b}_{3}$. Figure 3.1c shows a schematic representation of the reciprocal space lattice. Here, the blue planes illustrate the hexagonal planes described by $\mathbf{b}_{1}$ and $\mathbf{b}_{2}$. Due to the $2 \mathrm{D}$ periodicity inside each hexagonal plane the scattering intensity in reciprocal space will be concentrated around the integer values of $h$ and $k$. Stacking faults, inherent to an RHCP structure, break the interlayer periodicity smearing out the diffraction features in rows along $l$. The diffraction amplitude of each layer in the colloidal crystal grain along $l$ is dependent on the layer position in direct space. 

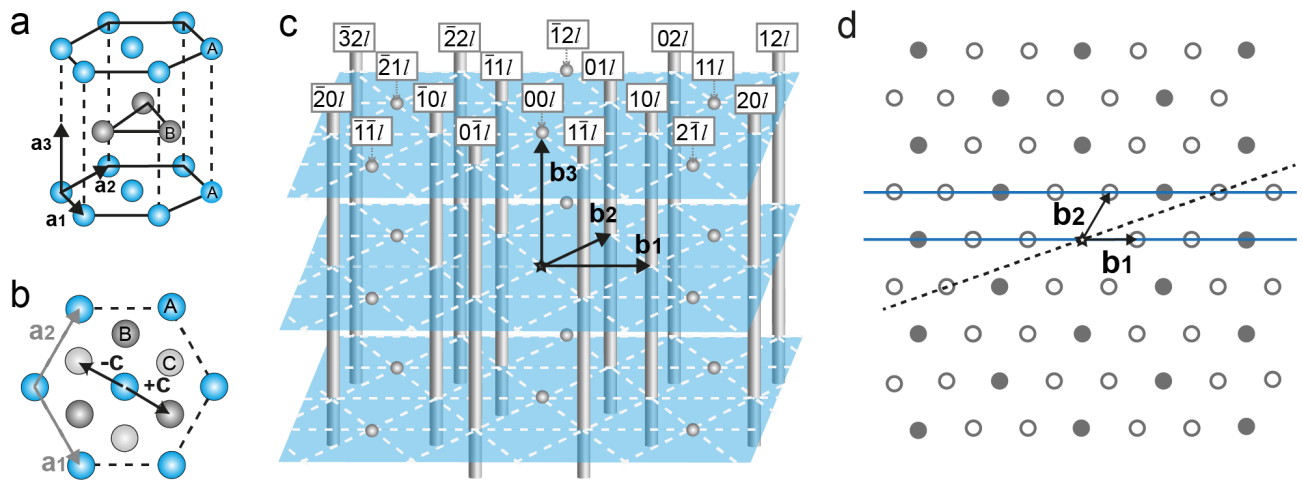

Figure 3.I. a) Hexagonal unit cell in direct space together with the basis vectors $\mathbf{a}_{1}, \mathbf{a}_{2}$ and $\mathbf{a}_{3}$. b) Top view of A, B, C layer positions and lateral displacement vector c. c) Sketch of the reciprocal lattice of randomly stacked hexagonal close-packed crystals described by vectors $\mathbf{b}_{1}, \mathbf{b}_{2}$ and $\mathbf{b}_{3}$. For specific $h k$ indices, as labelled, the intensity along $l$ is concentrated into distinct Bragg peaks (grey spheres) for $h-k$ divisible by 3 and smeared into Bragg rods (grey rods) for $h$ - $k$ not divisible by 3 . d) Top view of reciprocal space lattice, filled symbols represent Bragg peak positions, open symbols represent Bragg rod positions. Dotted line shows the cut of Ewald sphere through the $21 \mathrm{l}$ rods of the pattern shown in Fig 3.2.a. Blue lines indicate the positions of the 2D slices through the constructed 3D reciprocal space of Fig. 3.2.b,c.

As described above, this will be shifted by the vector $\mathbf{a}_{3}$ in the direction normal to the layers and by \pm c laterally.

Correspondingly, in reciprocal space the contribution of the $n$-th layer to the diffraction amplitude will receive a $\mathbf{q}$-dependent phase shift along $l$. The phase factor is described by, where $\Delta \varphi_{n}$ is the phase shift caused by the lateral displacement $\pm \mathrm{c}$ given by a recurrent relation;

$$
\Delta \varphi_{n}=\Delta \varphi_{n-1} \pm 2 \pi \frac{(h-k)}{3}
$$

Using Eq. (3.1) the structure factor $S(l)$ of a crystal containing $N$ hexagonal layers along $l$ is given by

$$
S(l)=\left|\sum_{n=0}^{N-1} \exp \left(i \Delta \varphi_{n}+2 \pi i n l\right)\right|^{2}
$$

here, the first layer is $n=0$ and always assigned an A position. It has to be noted that all layers are assumed to be of the same size.

For specific $h k$ indices such that $h-k$ is divisible by 3 , the phase shift $\Delta \varphi_{n}$ is always a multiple of $2 \pi$ and $S(l)$ reduces to the usual result, ${ }^{[1]}$

$$
S(l)=\left|\frac{\sin (\pi N l)}{\sin (\pi l)}\right|^{2}
$$

with well-defined Bragg peaks around integer values of $l$ independent of the stacking sequence and thus resembling a perfectly regular crystal with $N$ planes. The positions of the Bragg peaks 
are represented by the grey spheres in Figure 3.1.c and are regularly spaced at integer values of $l$.

For the other $h k$ indices, $h-k$ not divisible by 3 , the $S(l)$ profile will sensitively depend on the exact sequence of the $N$ layers as given by Eq.(3.2) and the intensity is concentrated into continuous rods along $l$, represented by the grey rods in Figure 3.1.c. For each stacking sequence a unique $S(l)$ profile is obtained that is periodic with over a range of $l=1$.

The positions of the Bragg spots and Bragg rods located at the specific $h k$ indices is seen more clearly in Figure 3.1.d where the top view of the reciprocal space is shown. The positions of the Bragg peaks are indicated with the filled symbols and the Bragg rods with the open symbols.

The total scattering intensity $I$ along $l$ for a colloidal crystal grain with a specific stacking sequence is the product of the form factor and the structure factor along $l$, given by the following equation,

$$
I(l) \propto P(l) S(l)
$$

where $P(l)$ is the form factor of a colloidal sphere with radius $R$ normalized to its volume similar to Eq. $(1.2)^{[21]}$,

$$
P_{h k}(l)=\left[\frac{3\left(\sin \left(q_{h k l} R\right)-q_{h k l} R \cos \left(q_{h k l} R\right)\right)}{\left(q_{h k l} R\right)^{3}}\right]^{2}
$$

where $q_{h k l}$ is the $q$ value along $l$ for a specific rod determined by its $h k$ location and given by;

$$
q_{h k l}=\sqrt{{q_{h k 0}{ }^{2}+q_{00 l}^{2}}^{2}}=\frac{2 \pi}{a} \sqrt{\frac{4}{3}\left(h^{2}+k^{2}+h k\right)+\frac{3}{2} l^{2}}
$$

where we assume that the inter-particle distance $a$ is $1 \%$ larger than the average particle diameter $(2 R)$, as previously shown for polydisperse colloidal spheres ${ }^{[22]}$.

It has to be noted that along $l$ the $P_{h k}(l)$ contribution to $I(l)$ depends on the specific $h k$ indices while the $S(l)$ contribution is only dependent on the stacking sequence. Therefore, groups of $h k l$ rods with the same $P_{h k}(l)$ contributions can be classified into a general family, for example all rods $10 l, 01 l,-10 l, 0-1 l,-11 l, 1-1 l$ belong to the $10 l$ family (See appendix A.3.1 for more details). 


\subsubsection{Results and discussion}

In the full $180^{\circ}$ coherent X-ray diffraction dataset collected from the selected colloidal crystal grain a number of Bragg peaks and distinct Bragg rods were observed. Figure 3.2.a shows a two dimensional (2D) diffraction pattern where the 001 Bragg peaks (as labelled) and two $21 l$ Bragg rods (indicated by arrows) are clearly visible. The darker square region in the middle of the diffraction pattern is the area covered by the semi-transparent beam stop, and the zero-intensity crossed lines originate from the gaps separating the four tiles of the MAXIPIX detector. For this sample orientation the X-ray beam propagates along the [450] direction of the colloidal crystal grain and the two $21 \mathrm{l}$ Bragg rods lie almost perfectly on the Ewald sphere. In the top view of the reciprocal lattice (Fig. 3.1.d) this is indicated by the black dotted line.

Using all 360 diffraction patterns the $3 \mathrm{D}$ reciprocal space distribution of scattered intensities was constructed. As expected for a crystal with stacking disorder, this revealed the presence of Bragg rods through the full 3D reciprocal space. Figure 3.2.b and 3.2.c show two extracted 2D slices along $l$ of the constructed 3D reciprocal space, where the black lines appear again due to the detector gaps. The locations of the projections in reciprocal space are indicated by the blue lines in the reciprocal lattice shown in Figure 3.1.d. In the 2D slices all the specific Bragg rods are labelled and these can be classified as the Bragg rod families: $10 l, 20 l$ and $21 l$.

Because the coherence length of the beam is larger than our crystal grain and the setup has sufficient angular resolution, we can resolve the fine structure of the Bragg rod. The specific modulations of intensity along $l$ are distinctly different from the results reported so far ${ }^{[7-10,23]}$. In those studies much larger colloidal crystals were investigated and the measured Bragg rod profiles, consisting of smears between the Bragg peaks, only provided information on the average stacking fault densities.

Bragg rod profiles were extracted from 2D slices through the constructed 3D reciprocal space
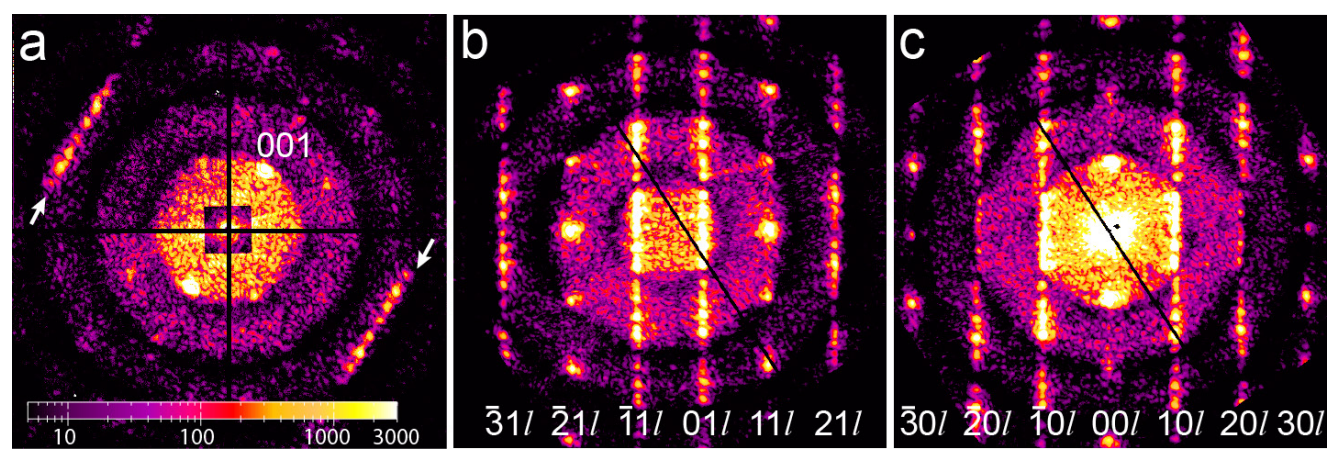

Figure 3.2. a) 2D Diffraction pattern measured along the [450] direction of the individual crystal grain, where the $00 \mathrm{I}$ Bragg peaks and two Bragg rods of the $2 \mathrm{l} l$ family are clearly visible (indicated by white arrows). b\&c) 2D slices taken through the full 3D reciprocal space constructed from the 360 diffraction patterns. These slices cut through the reciprocal space lattice at the Bragg peak and rod positions as illustrated by the blue lines in the top view of the reciprocal space lattice of Fig. 3.I.d and show a number of different Bragg rods. The slice shown in b) does not cross through the origin of the reciprocal space and therefore does not show the direct beam feature. 


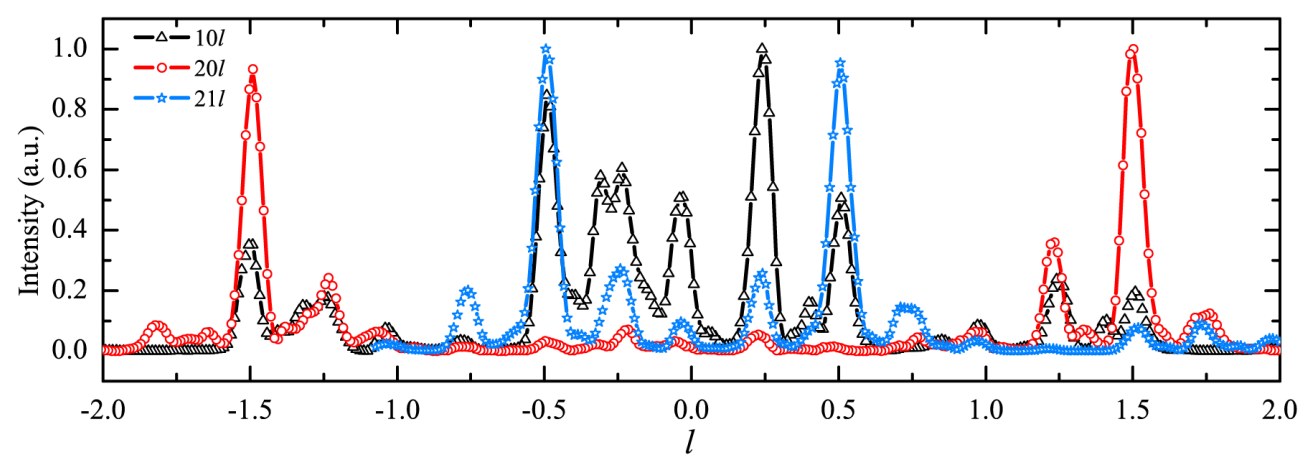

Figure 3.3. Normalized experimental profiles of the $10 l, 20 l$ and $21 l$ Bragg rod families that show distinct intensity modulations along $l$. One can see a strong correlation in the positions of the peaks, which originate from the same $S(l)$. The peak height, however, are different due to the form factor $P_{h k}(l)$, that depends on the $h k$ indices.

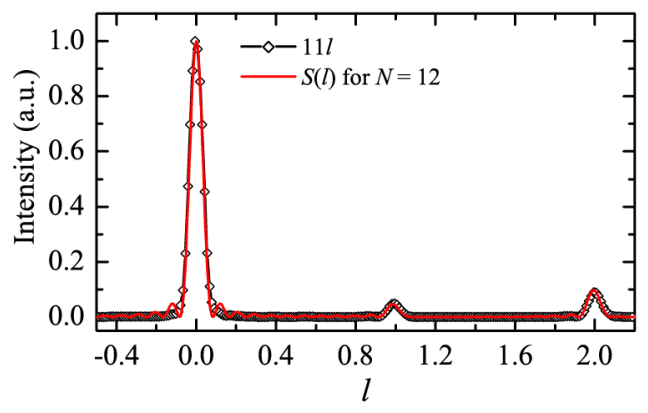

Figure 3.4. Normalized experimental Bragg peak profile along the $I I l$ direction fitted with a peak model for $N=12$ layers (Eq.(3.3)).

for all visible Bragg rods. The $l$ values of the rods were determined by locating the middle of the rods with respect to the incident beam center and scaled with the q-value of the 001 Bragg peak as this is located at $l=1$. Representative normalized intensity profiles along the Bragg rod for the $10 l$, $20 l$ and $21 l$ families are plotted in Figure 3.3, where the intensity modulations can clearly be seen. It is observed that for each rod profile the peak positions are similar while the peak amplitudes differ significantly. This is as expected because for each combination of $h k$ indices the structure factor $S(l)$ should be the same, while the form factors $P_{h k}(l)$ contribution is different.

The total number of layers in the studied crystal grain $N$ was determined by fitting the width of the Bragg peaks along the $11 l$ rod using Eq. (3.3) and it was found that $N=12$. The measured $11 \mathrm{l}$ rod profile and the corresponding fitting curve, showing good agreement in terms of peak positions and shape, are displayed in Figure 3.4. It has to be noted that Eq. (3.3) assumes that all layers are of the same size. In practice the colloidal crystal grain is obtained by fracturing a dried sediment and it is very unlikely that the layers contain the same number of particles (see also Fig. 2.6). Layers with a smaller size will contribute less to the peak broadening and therefore the actual 
number of layers in the grain $N$ might be larger.

Using the model for the intensity distribution $I(l)$ described by Eq. (3.4) the Bragg rod profiles for different stacking sequences with $N=12$ were calculated. To obtain quantitative agreement between model and experiment the intensity between $-1<l<1$ was used for analysis, with exception of the $20 l \mathrm{rod}$, where the range $0<l<2$ was used due to low intensity at $|l|<1$.

After careful examination of all possible stacking sequences we found two sequences that yielded the best match to the experimental profiles. Figure 3.5 shows the experimental Bragg rod profiles for $10 l, 20 l$ and $21 l$ Bragg rod families and the best fits calculated for the two found sequences. Surprisingly, both sequences are periodic over 4 layers and belong to the so-called Double Hexagonal Close Packed (DHCP) structure. The DHCP structure has a stacking sequence of $\mathrm{ABCBABCB}$.... and is schematically represented in Figure 3.5.d. The DHCP sequence is typical for some of the rare earth metals ${ }^{[24]}$ but has not been reported for colloidal crystals of spheres before.
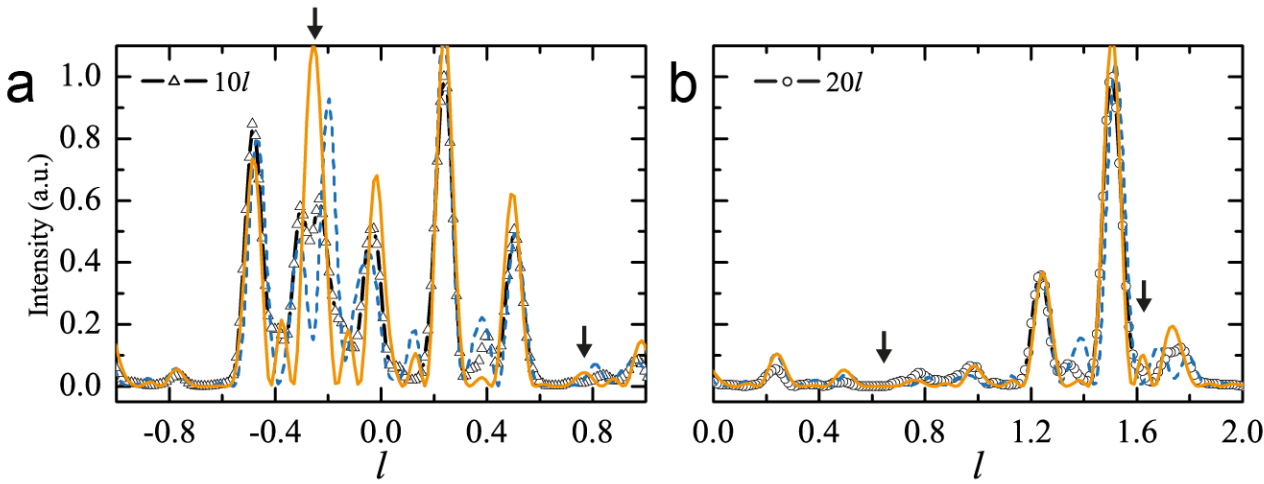

— ABCBABCBABCB (perfect DHCP) - - - ABCBABCBCACB (1 defect DHCP)
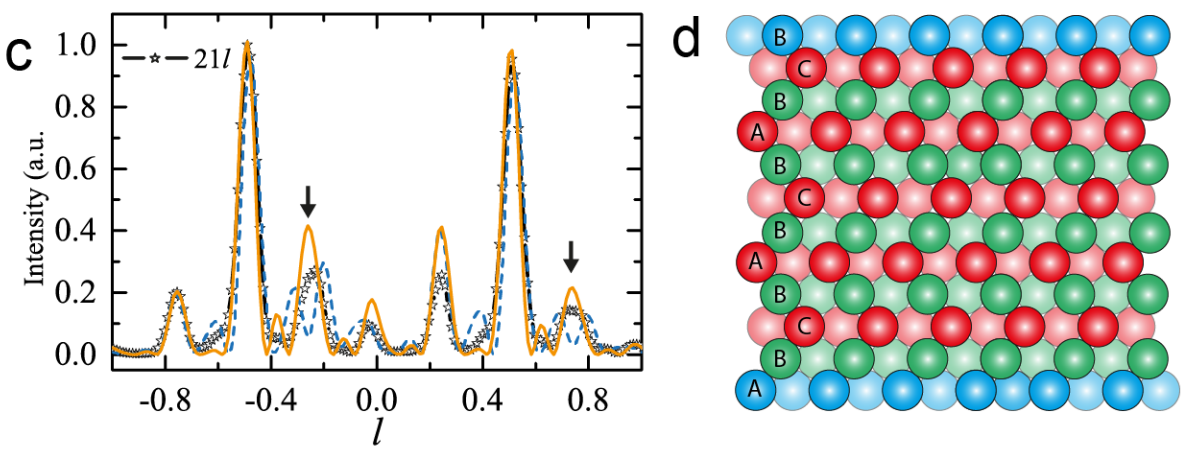

Figure 3.5. Normalized experimental Bragg rod profiles (black line + symbol) of a) $10 \mathrm{lb}$ ) $20 \mathrm{l}$ and c) $2 \mathrm{Il}$ and modelled profiles for two DHCP structures, perfect sequence (orange line) and a sequence with single stacking fault (blue dashed line). The specific layer sequence is represented by the $A, B$ and $C$, sequence, where a layer is in an HCP environment (red) or an FCC environment (green). Arrows indicate the region where the two DHCP model profiles mismatch the most and have a single or double peak, respectively. d) Schematic representation of the side view of a perfect DHCP stacking sequence for 12 layers. 
The first stacking sequence, $\mathrm{ABCBABCBABCB}$, is a perfect $\mathrm{DHCP}$ structure that consists of every second layer at a $\mathrm{B}$ position in an FCC environment and alternating A/C layers in an $\mathrm{HCP}$ environment. The second sequence, $\mathrm{ABCBABCBCACB}$, is similar to the first one with the exception of the two underlined layers. These two layers cause a stacking defect in the perfect DHCP sequence, and change the repeating B layer to a repeating C layer. The calculated $I(l)$ profiles for the two considered DHCP sequences are very similar in terms of peak positions, shapes and amplitudes and describe all three experimental Bragg rod profiles overall very well.

The main difference between the two DHCP profiles is found periodically at $l=-0.25,0.75$, 1.75 as indicated with arrows in Fig. 3.5, where a single peak is split into a double peak by the stacking defect. For all three Bragg rod profiles this is the location where both model profiles fail to describe the experimental data. Apparently the distribution of stacking defects in the studied crystal is more complex than considered in the stacking model. For instance, each layer can consist of a different number of particles or the layers can exhibit in-plane stacking disorder ${ }^{[3]}$. In the latter case two stacking positions are present inside a single hexagonally close packed layer, such as islands of $\mathrm{A}$ and $\mathrm{C}$ layers coexisting on top of a B layer. The result is two different stacking sequences in a single grain that will influence $S(l)$. The other small deviations between the experimental data and the model could be explained by our simplified approach to determine $I(l)$.

The overall good agreement obtained using the simple model for $I(l)$ shows that the DHCP structure is the dominant packing arrangement of the colloidal spheres present in the studied crystal grain. (For more details on sensitivity of the Bragg rod model see Appendix A.3.2). This is a remarkable result because the DHCP structure has not been observed before in colloidal crystals of spheres. Previous studied have indicated that the growth of a sedimentary crystal with an RHCP structure probably occurs via the sequential nucleation of hexagonal layers ${ }^{[3]}$. The interactions are limited to neighboring layers and therefore, the DHCP structure could just be one of the many random coincidental realizations of the RHCP structure. Alternatively, it could be an effect of the drying process in which capillary forces of significant strength act on the colloids ${ }^{[25]}$. 


\subsection{D reconstruction}

The scattering data described above was also analyzed using phase retrieval algorithms by Shabalin et al. ${ }^{[26]}$. They first constructed the full 3D diffraction data including the diffuse scattering as shown in Figure 3.6.a. This data was then converted into the 3D electron density of the colloidal crystal grain. Figure 3.6.b shows an iso-surface plot of the reconstructed volume, while Figure 3.6.c shows a slice through the reconstructed volume. The positions of the individual colloidal particles can be clearly distinguished. By analyzing the projection of one of the crystallographic directions the number of layers and the stacking sequence could be determined as shown in Figure 3.6.d. The analysis showed the presence of 14 layers in the crystal with a DHCP stacking sequence of ABCBABCBABCBCA. This is an almost perfect DHCP stacking sequence with only a small stacking fault in the last two layers (underlined). The stacking sequence is very similar to the DHCP stacking and the 'defect'-DHCP stacking that were revealed with the Bragg rod analysis for 12 layers.

The difference in the stacking sequence found with the Bragg rod analysis method with respect to that from the reconstructed $3 \mathrm{D}$ volume via phase retrieval algorithms can be explained by the finite size of the grain. In the Bragg rod model we have assumed equal layer sizes, while the reconstructed grain clearly shows that the layers at the top and bottom of the grain are much smaller than those in the center. Figure 3.7 shows the calculated Bragg rod profile for the new stacking sequences compared to the experimental Bragg rod profiles which correspond very well.

\subsection{Conclusions}

In the coherent $\mathrm{X}$-ray diffraction data of a single colloidal crystal grain distinct Bragg peaks and Bragg rod were observed. Characteristic intensity modulations along the Bragg rods indicated the presence of stacking faults in the sequence of hexagonally close packed layers. The intensity fluctuations were described by a model that takes into account the stacking sequence of a finite number of hexagonally close packed layers. Using the model two stacking sequences of the DHCP type, where FCC and HCP stacking alternate periodically, were found to match the experimental data. The slight mismatch between a perfect DHCP and a single stacking fault DHCP, and the experimental data, suggests that the grain may contain a stacking fault in a dominant DHCP structure. The presence of a DHCP structure is remarkable as it has not been observed for colloidal crystal of spheres before.

Although we have taken a relatively simple approach for the analysis of the stacking sequence, our conclusions are consistent with preliminary results of our independent analysis of 3D electron density distribution in real space, obtained using a phase retrieval algorithm ${ }^{[26]}$. This agreement shows that our method is a feasible new route for the analysis of finite-size objects. The proposed method can be applied to more general classes of finite-size structures studied with highly brilliant $3^{\text {rd }}$ generation synchrotron sources to extract crucial structure information from the diffraction data. 

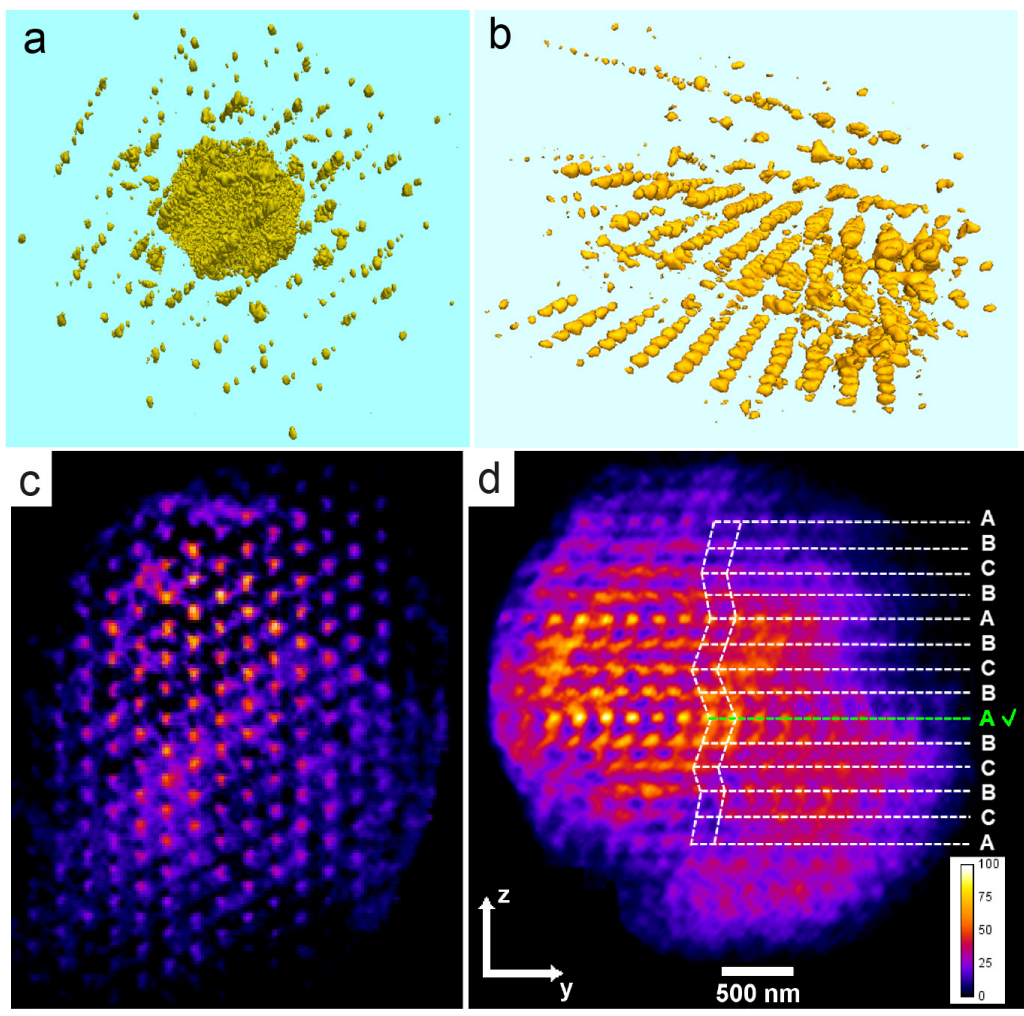

Figure 3.6. a) 3D scattered intensity in reciprocal space. b) Iso-surface in reconstructed volume of the colloidal crystal grain. c) Slice through one single crystalline plane. d) Projection of reconstructed electron density on one orthogonal direction, showing the stacking sequence.
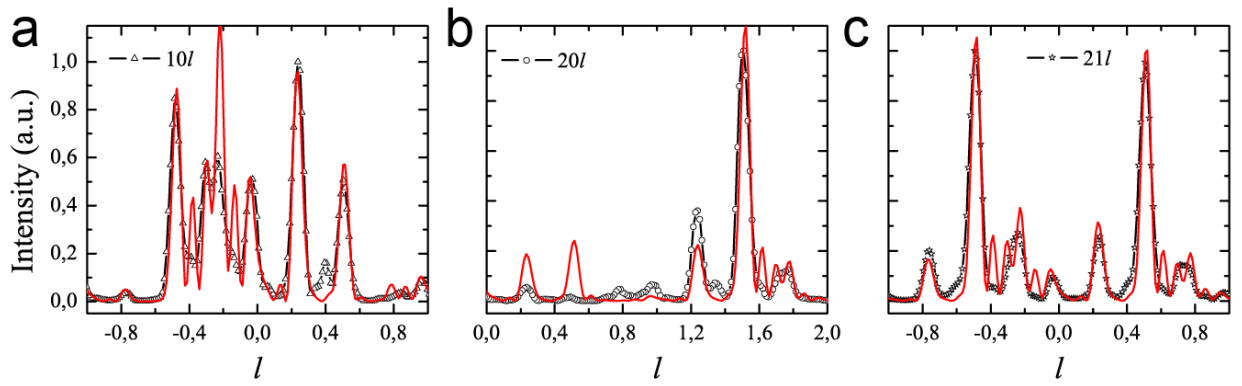

Figure 3.7. Normalized experimental Bragg rod profiles (black line + symbol) of a) $l 0 l$ b) $20 l$ and c) $2 \mid l$ and modelled profile for defect-DHCP structure with 14 layers as determined from the reconstructed crystal grain (red line). 


\section{Acknowledgements}

This work is the result of a collaboration with the Vartanyants' group from Hasylab at DESY, Hamburg. I would like to thank Ivan Vartanyants, Anatoly Shabalin and Johannes Gulden for the close collaboration and useful discussions. Alexey Zozulya, Ulf Lorenz, Dmitry Dzighaev, Oleg Gorobstov, Ruslan Kurta, Andrej Singer, Oleksandr Yefanov and Dmitro Byelov are acknowledged for their contributions to the CXDI experiment. The P10 beamline team of PETRAIII, DESY, Hamburg is thanked for providing technical support. DESY is acknowledged for allocating the beamtime. Jan Hilhorst is thanked for useful suggestions. Part of this work is reproduced with permission of the International Union of Crystallography from ref [27].

\section{Appendices}

\section{A.3.I. $P_{h k}(I)$ and $S(I)$ contributions at different $h k$ indices}

In our model the scattering intensity depends on the form factor $P_{h k}(l)$ and the structure factor $S(l)$ and these have different effects on the full intensity $I_{h k}(l)$ along a Bragg rod. $P_{h k}(l)$ is dependent on the specific $h k$ indices of the Bragg rod while $S(l)$ is independent of the $h k$ indices and only determined by the stacking sequence, see Eq.(3.2). Figure 3.8.a shows the normalized $P_{h k}(l)$ profiles for the different indices $h k(10), h k(20)$ and $h k(21)$. It is clear that the maxima and minima are located at different $l$ values. The $S(l)$ profile is shown in Figure 3.8.b for a DHCP stacking sequence and shows a specific modulation of the intensity, with a periodicity of $l=1$. In Figure 3.8.c the final intensity $I_{h k}(l)=P_{h k}(l) S(l)$ (Eq.3.4) is plotted for the specific $h k$ indices. This shows the strong influence the specific $P_{h k}(l)$ on the final peak profile of $S(l)$ and explains the large difference of the Bragg rod profiles for the different families shown in Figure 3.3.

\section{A.3.2 Sensitivity of Bragg rod Model for stacking sequence}

To illustrate the sensitivity of the intensity profile along the Bragg rods to the exact stacking sequence, and thus the strength of this analysis route, we show two DHCP structure with two other small changes in the DHCP sequences for the $21 l$ rod in Figure 3.9. The two profiles sequences are $\mathrm{ABCBABABABCB}$ and $\mathrm{ABCBABCBABCA}$, in which the differences with the DHCP stacking are the middle and last layer, respectively. These both change the number of FCC and HCP layers in the structure and clearly have a dramatic effect on the final model $I_{h k}(l)$, where peak position, shape and intensity are clearly mismatched with the experimental data. 

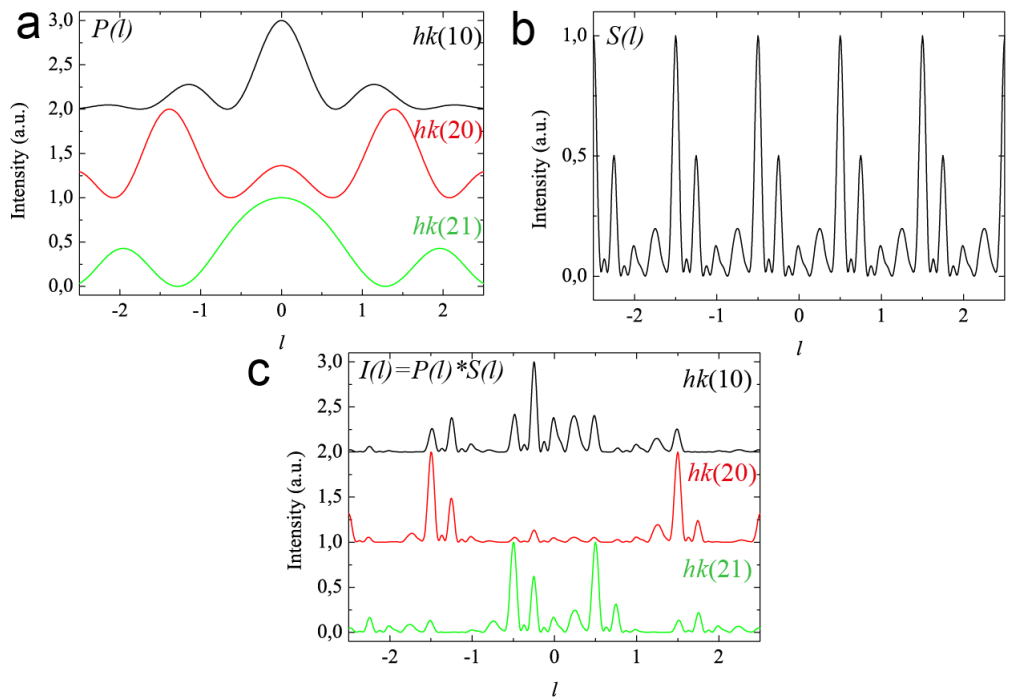

Figure 3.8. a) Normalized profiles of $P(l)$ for the different $h k$ indices of the Bragg rods, the graphs are offset by I for clarity. b) Normalized profile of $S(l)$ for a DHCP stacking sequence, which is independent of the $h k$ indices. c) Final normalized intensity profiles $I(l)$ for different $h k$ indices. Clearly, the effect of $P_{h k}(l)$ and hence the $h k$ indices of the rod is of large influence.

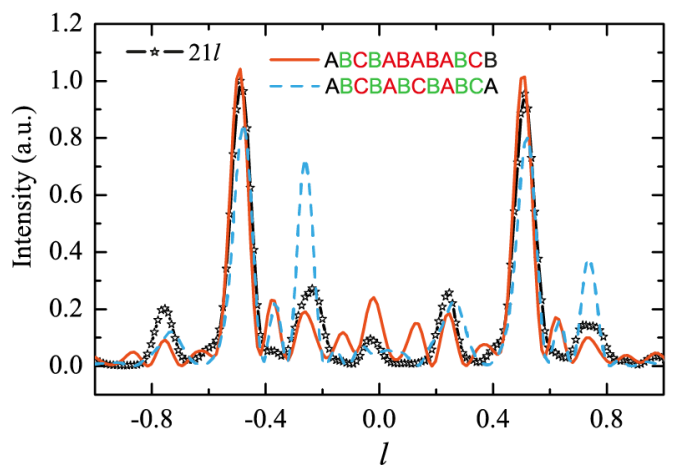

Figure. 3.9. Normalized experimental $2 \mid l$ rod profile with modelled profiles for two different DHCP stacking sequences, with a different middle or end layer than a perfect DHCP structure, showing the sensitivity of the model to a sequence change. 


\section{References}

1. A. Guinier, X-Ray Diffraction in Crystal, Imperfect Crystals, and Amorphous Bodies, Dover, New York, 1994.

2. A. J. C. Wilson, Proc. R. Soc. A, 1942, 180, 277-285.

3. J. M. Meijer, V. W. A. de Villeneuve and A. V. Petukhov, Langmuir, 2007, 23, 3554-3560.

4. P. G. Bolhuis, D. Frenkel, S. C. Mau and D. A. Huse, Nature, 1997, 388, 235-236.

5. A. D. Bruce, N. B. Wilding and G. J. Ackland, Phys. Rev. Lett., 1997, 79, 3002-3005.

6. S. Mau and D. Huse, Phys. Rev. E, 1999, 59, 4396-4401.

7. H. Versmold, S. Musa, C. Dux and P. Lindner, Langmuir, 1999, 15, 5065-5067.

8. A. V. Petukhov, I. P. Dolbnya, D. G. A. L. Aarts, G. J. Vroege and H. N. W. Lekkerkerker, Phys. Rev. Lett., 2003, 90, 028304 .

9. J. Hilhorst, V. V. Abramova, A. Sinitskii, N. A. Sapoletova, K. S. Napolskii, A. A. Eliseev, D. V. Byelov, N. A. Grigoryeva, A. V. Vasilieva, W. G. Bouwman, K. Kvashnina, A. Snigirev, S. V. Grigoriev and A. V. Petukhov, Langmuir, 2009, 25, 10408-10412.

10. D. V. Byelov, J. Hilhorst, A. B. G. M. Leferink op Reinink, I. Snigireva, A. Snigirev, G. B. M. Vaughan, G. Portale and A. V. Petukhov, Phase Transitions, 2010, 83, 107-114.

11. J. Gulden, O. M. Yefanov, A. P. Mancuso, V. V. Abramova, J. Hilhorst, D. Byelov, I. Snigireva, A. Snigirev, A. V. Petukhov and I. A. Vartanyants, Phys. Rev. B, 2010, 81, 224105.

12. J. Gulden, O. M. Yefanov, A. P. Mancuso, R. Dronyak, A. Singer, V. Bernatova, A. Burkhardt, O. Polozhentsev, A. Soldatov, M. Sprung and I. A. Vartanyants, Opt. Express, 2012, 20, 4039-4049.

13. J. R. Fienup, Appl. Opt., 1982, 21, 2758-2769.

14. V. Elser, JOSA A, 2003, 20, 40-55.

15. K. A. Nugent, Adv. Phys., 2010, 59, 1-99.

16. P10 beamline, http://photon-science.desy.de/facilities/petra_iii/beamlines/p10_coherence_applications/index_eng. html, visited on 30/01/2014.

17. A. V. Zozulya, S. Bondarenko, A. Schavkan, F. Westermeier, G. Gruebel and M. Sprung, Opt. Express, 2012, 20, 18967-18976.

18. A. Snigirev, V. Kohn, I. Snigireva and B. Lengeler, Nature, 1996, 384, 49-51.

19. V. Kohn, I. Snigireva and A. Snigirev, Opt. Commun., 2003, 216, 247-260.

20. M. Drakopoulos, A. Snigirev, I. Snigireva and J. Schilling, Appl. Phys. Lett., 2005, 86, 014102.

21. J. S. Pedersen, Modeling of Small-Angle Scattering Data from Colloids and Polymer Systems, in Neutrons, X-rays and Light: Scattering Methods Applied to Soft Condensed Matter, ed. P. Linder and T. Zemb, Elsevier Science B.V., 2002, pp.391-420.

22. A. V. Petukhov, J. H. J. Thijssen, D. C. 't Hart, A. Imhof, A. van Blaaderen, I. P. Dolbnya, A. Snigirev, A. Moussaid and I. Snigireva, J. Appl. Cryst., 2006, 39, 137-144.

23. A. A. Eliseev, D. F. Gorozhankin, K. S. Napolskii, A. V. Petukhov, N. A. Sapoletova, A. V. Vasilieva, N. A. Grigoryeva, A. A. Mistonov, D. V. Byelov, W. G. Bouwman, K. O. Kvashnina, D. Y. Chernyshov, A. A. Bosak and S. V. Grigoriev, JETP Letters, 2009, 90, 272-277.

24. W. B. Pearson, Handbook of Lattice Spacings and Structure of Metals, Pergamon Press, Oxford, 1967.

25. N. Denkov, O. Velev, P. Kralchevski, I. Ivanov, H. Yoshimura and K. Nagayama, Langmuir, 1992, 8, 3183-3190.

26. A. Shabalin, J. M. Meijer, R. Dronyak, O. M. Yefanov, A. Singer, R. P. Kurta, U. Lorenz, O. Gorobstov, D. Dzhigaev, S. Kalbfleisch, A. V. Zozulya, J. Gulden, T. Salditt, M. Sprung, A. V. Petukhov and I. A. Vartanyants, in preparation, 2014.

27. J. M. Meijer, A. Shabalin, R. Dronyak, O. M. Yefanov, A. Singer, R. P. Kurta, U. Lorenz, O. Gorobstov, D. Dzhigaev, J. Gulden, D. V. Byelov, A. V. Zozulya, M. Sprung, I. A. Vartanyants and A. V. Petukhov, J. Appl. Cryst., 2014, 47, 1199-1204. 


\section{CHAPTER 4}

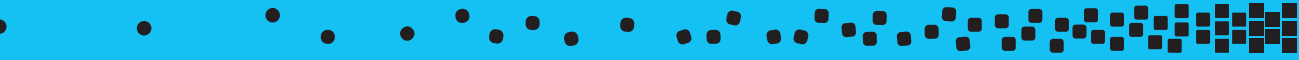

INDUCING DEFECTS IN COLLOIDAL CRYSTALS WITH THERMOSENSITIVE PNIPAM PARTICLES

Abstract

Thermosensitive colloidal PNIPAm particles are employed to induce defect structures into a colloidal crystal. The PNIPAm particle size can be controlled by temperature due to collapse of the polymer network above the lower critical solution temperature (LCST). By embedding these PNIPAm particles into a colloidal crystal formed by non-thermoresponsive particles vacancy-like structures could be induced above the LCST and interstitial structures below the LCST. Our aim is to study the three-dimensional defect diffusion by tracing individual particles and to gain insight into the mechanism involved. Here, we present the observed defect diffusion induced in a mixed system of fluorescently labeled PNIPAm and PNIPAm-AAc particles in which defect diffusion was achieved. 


\section{I. Introduction}

Many materials are crystalline, with their building blocks (atoms or molecules) arranged into ordered repeating arrays extending in all three dimensions. However, real crystals never have perfect crystalline ordering but contain different types of defects. ${ }^{[1,2]}$ Defects are structural imperfections interrupting the ordering in a crystal that determine fundamental crystal properties, such as, their mechanical, electric and optical properties ${ }^{[1]}$. There are several kinds of crystal imperfections; point, line, surface and volume defects of which the behavior and effects are intimately related. Although these defects can be disadvantageous, defects can also be added on purpose. It is known that the electronic behavior of materials can be influenced by adding specific impurities ${ }^{[3]}$, or the mechanical behavior strengthened by work hardening, a process in which dislocations get pinned ${ }^{[2]}$.

Because crystals and their defects play an very important role in the behavior of materials, they have been the focus of numerous studies and the microscopic structure of defects and their effects are well documented ${ }^{[1,2]}$. However, point defects such as vacancies and interstitials can also diffuse and interact with each other as described by the elastic dislocation theor ${ }^{[4]}$. Clustering of the defects can result in dislocation loops with one-dimensional diffusion controlled by the collective motion of dislocations ${ }^{[5,6]}$. Experimental defect studies on a single particle level are challenging because of the atomic scale. Defect cores have a similar size as the atoms $(\AA)$ and the strains fields are not much bigger $(\mathrm{nm})$. Therefore, high resolution techniques have to be used to visualize the atoms and the dislocations ${ }^{[7-9]}$.

Although challenging, defect diffusion on an atomic scale was recently investigated with high resolution transmission electron microscopy (HR-TEM). Arakawa et al. showed that interstitial-type dislocation loops undergo thermally induced one-dimensional (1D) diffusion in BCC iron ${ }^{[10]}$. Another notable example is the work of Matsukawa et al. that demonstrated the thermally induced one-dimensional diffusion of vacancy-type dislocation loops in gold $^{[11]}$. In addition, they observed a direct transformation of a loop into a stacking fault tetrahedron, a typically observed defect in FCC crystals $^{[2]}$. These studies raise new questions about the mechanisms of diffusion and the interactions on a single particle level.

One can overcome the length- and time scale problem by using a model system of colloidal particles, that have been widely used since the pioneering work of Perrin more than a century ago $^{[12]}$. Colloidal particles offer the advantage of being directly visible with optical microscopy and can be individually traced over time as the time-scales involved are on the order of seconds ${ }^{[13]}$. In addition, due to the weak interactions between particles, the formation of dislocations costs relatively little energy ${ }^{[14-16]}$. There are several colloidal crystal studies that focused on defect diffusion. For example, Pertsindis et al. studied the diffusion of defects induced by an optical tweezer within a two-dimensional (2D) array of polystyrene spheres interacting via screened coulomb interactions ${ }^{[17,18]}$. Eisenmann et al. followed dislocation pairs in superparamagnetic colloidal particles confined to the air-water interface ${ }^{[19]}$. Computer simulations combined with experiments later showed that diffusion of defect strings will occur in $1 \mathrm{D}^{[20-24]}$.

All these studies have been confined to $2 \mathrm{D}$ systems while the defect diffusion in atomic 
a

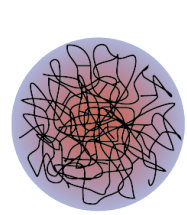

$25^{\circ} \mathrm{C}$
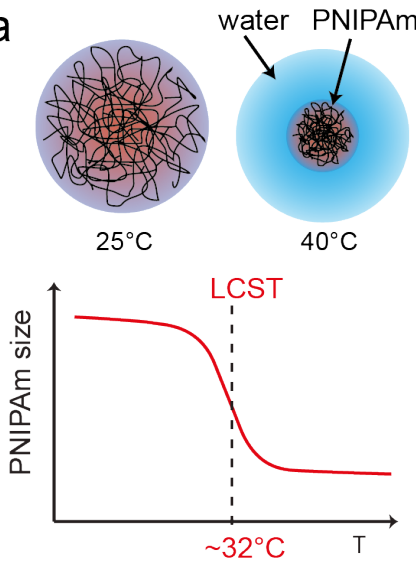

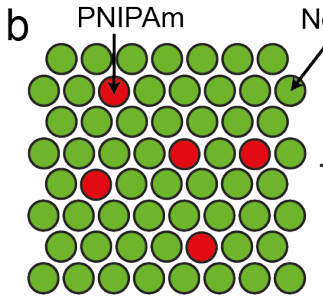

Non-responsive

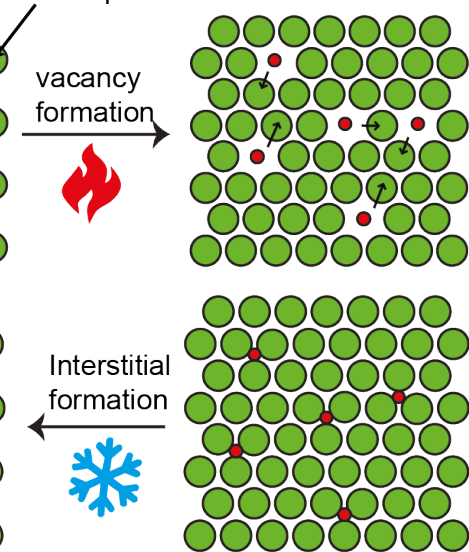

Figure 4.I. a) Schematic representation of VPT of cross-linked PNIPAm particles. With increasing temperature the particle goes from a swollen state $\left(25^{\circ} \mathrm{C}\right)$ to a shrunken state $\left(40^{\circ} \mathrm{C}\right)$, caused by dominating hydrophobic interactions that expel the water from the polymer network. The LCST is located around $32{ }^{\circ} \mathrm{C}$. b) Schematic representation of the proposed effect of incorporating PNIPAm (red) particles into a non-responsive particle (green) crystal upon heating and cooling, allowing the controlled creation of vacancy-like and interstitial-like structures for defect diffusion studies.

crystals is of course a $3 \mathrm{D}$ process. To study defects in a $3 \mathrm{D}$ crystal one would have to induce defects into a hard sphere systems, where number density and volume fraction cannot be changed easily. There is, however, a colloidal systems consisting of poly-N-isopropyl acrylamide (PNIPAm) spheres that can change its diameter up to $90 \%$ in situ by changing the dispersion temperature ${ }^{[25]}$. The NIPAM monomer has a lower critical solution temperature (LCST) of $32{ }^{\circ} \mathrm{C}$ in water. Below this temperature the hydrophilic interactions of the polymer dominate and particles are swollen with water. Above the LCST, the hydrophobic interactions dominate and water is expelled from the particle network ${ }^{[26]}$. This volume phase transition (VPT) process and effect on particle size is schematically shown in Figure 4.1.a. PNIPAm particles have already been used for studies of annealing and controlled melting at crystal grain boundaries ${ }^{[27,28]}$ and for the determination of the dislocation width as a function of particle potential ${ }^{[16]}$.

Here, we explore the use of PNIPAm particles as defect inducers. Our idea is to investigate whether the thermosensitive behavior of the PNIPAm particles can be used to induce defects in situ in a non-thermoresponsive colloidal crystal by tuning their size ratio with temperature. Figure 4.1.b. shows a schematic representation of this proposed process. Different types of defects could be formed upon a temperature change; vacancy-like defects upon an temperature increase and interstitial-like defects with a temperature decrease. It is expected that due to the increased available free volume or internal stress, created by the vacancies and interstitials structures respectively, defect diffusion will be induced. By following this process with confocal laser scanning microscopy (CLSM) in time and space, we can obtain insight into the diffusion process and the mechanisms involved. 
We have first explored the synthesis of fluorescently labeled PNIPAm particles, a requirement that has to be met for the use of the CLSM. Next, the structures formed by the fluorescently labeled PNIPAm and silica particles were investigated and showed that this combination is not optimal. Therefore, two different PNIPAm systems were used of which one has a reduced temperature response by addition of acrylic acid (AAc) to the particle network ${ }^{[29]}$. Heating and cooling studies of the two PNIPAm systems were performed separately and in combination. This revealed a number of problems of the chosen system, including that the VPT of the PNIPAm particles did not induce vacancy-like structures upon heating. However, the PNIPAm particles did surprisingly induce defect diffusion upon cooling. We discuss the observed defects, their diffusion and the possible routes to improve the system.

\subsection{Experimental}

\subsubsection{PNIPAm synthesis}

PNIPAm particles were prepared by surfactant-free emulsion polymerization in an aqueous solution $^{[25]}$. Typically, $1.5 \mathrm{~g}$ N-Isopropylacrylamide (NIPAm, Sigma Aldrich, 97\%) and $40 \mathrm{mg}$ cross-linker N,N'-Methyelenebisacrylamide (BIS, Sigma Aldrich, 98\%) were dissolved in 100 $\mathrm{mL}$ water or buffer and transferred into a $250 \mathrm{~mL}$ round-bottom flask. For fluorescent labeling a dye solution was prepared consisting of $15 \mathrm{mg}$ fluoresceine isothiocyanate (FITC, Aldrich, Isomer I, 90\%) and $40 \mu \mathrm{L}$ 3-amino-propene (Acros, 98\%) in $10 \mathrm{~mL} 10^{-4} \mathrm{M} \mathrm{NaOH}$ (Merck, 99\%) that was flushed with nitrogen. The dye solution was added to the reaction mixture followed by heating to at $70{ }^{\circ} \mathrm{C}$ under reflux cooling, stirring under a gentle nitrogen stream for $30 \mathrm{~min}$. When the temperature was stable, a radical initiator solution consisting of $40 \mathrm{mg}$ potassium persulfate (KPS, Acros, 99\%) or ammonium persulfate (APS, Acros, 98\%) was added to the mixture. The reaction mixture was kept at $70{ }^{\circ} \mathrm{C}$ for at least $4 \mathrm{~h}$ to complete the reaction. Afterwards, the mixture was allowed to cool while maintaining stirring. Excess monomer was removed by means of repeated centrifugation (Beckman Coulder Allegra X-12R) at 2500 r.p.m. for at least 19 hours and redispersed in water. For all experiments water from a Millipore system was used.

Table 4.1 shows the exact composition of the two PNIPAm particles, PNIPAm-AP and PNIPAm used for the experiments in this study. The PNIPAm-AP particles were fluorescently labelled with fluorescein. The PNIPAm particles were synthesized with at $\mathrm{pH} 4$ (citrate buffer) and additional $\mathrm{NaCl}$ in solution to stabilize the particles during growth.

Table 4.I. Details of the composition of the three PNIPAm particles

\begin{tabular}{lccccc}
\hline Particle & $\begin{array}{c}\text { NIPAm } \\
(\mathbf{m m o l})\end{array}$ & $\begin{array}{c}\text { BIS } \\
(\mathbf{m m o l})\end{array}$ & $\begin{array}{c}\text { Co-monomer } \\
(\mathbf{m m o l})\end{array}$ & $\begin{array}{c}\text { Initiator } \\
(\mathbf{m m o l})\end{array}$ & $\begin{array}{c}\text { Dye } \\
(\mathbf{m m o l})\end{array}$ \\
\hline PNIPAm-AP & 13.5 & 0.27 & AP, 0.54 & KPS, 0.21 & FITC, 0.04 \\
PNIPAm & 18.03 & 0.28 & - & APS, 0.20 & - \\
PNIPAm-AAc & 12.01 & 0.27 & AAc, 1.33 & KPS, 0.15 & NBD-MAEM \\
\hline
\end{tabular}


An additional PNIPAm particle system, PNIPAm-AAc, was provided by J. Hilhorst. These PNIPAm particles were copolymerized with acrylic acid (AAc) and dyed with 4-methylamoniethylmethacrylate-7-nitrobenzo-2-oxa-1,3-diazol (NBD-MAEM). The composition of the PNIPAm-AAc particles is also sown in Table 4.1. The presence of the acrylic acid monomer in the microgel network adds $\mathrm{pH}$-dependent size behavior to the spheres, as well as additional charge stabilization at high temperature and $\mathrm{pH}^{[29]}$.

\subsubsection{PNIPAm characterization}

The size of the particles as a function of temperature and zeta-potential were analyzed with static and dynamic light scattering (SLS and DLS). Samples consisted of very diluted particle dispersions that were filtrated with a $5 \mu \mathrm{m}$ Millipore filter to remove dust and aggregates, before being placed into a sample cuvette.

SLS measurements were performed on a custom built setup, with a temperature controlled toluene bath and an $\mathrm{Hg}$ lamp as a light source. Monochromatic light of $578 \mathrm{~nm}$ was obtained using a band-pass filter and measurements were performed over a range of $20^{\circ}$ to $120^{\circ}$, with steps of $1^{\circ}$ and a temperature range of $25-39^{\circ} \mathrm{C}$. The radius of gyration, $R_{g}$, was determined at different conditions ( $\mathrm{pH}$, ionic strength). DLS and zeta-potential measurements were done with a Malvern Zetasizer Nano at $173^{\circ}$ in back scattering geometry. The light source used was He-Ne laser at a wavelength $\lambda=633 \mathrm{~nm}$.

\subsubsection{Sample preparation of mixtures}

Mixtures of PNIPAm-AP and silica spheres were prepared by mixing PNIPAm-AP stock dispersion (1 wt\%) with the silica stock (3.3 wt\%). PNIPAm and PNIPAm-AAc particle mixtures were prepared by first increasing the concentration of the particle stock dispersions by centrifugation at 2500 r.p.m (Beckman Coulder Allegra X-12R) for $19 \mathrm{~h}$ and removal of the supernatant. For the PNIPAm particles the temperature was raised to $40{ }^{\circ} \mathrm{C}$ to speed up the sedimentation. The volume fraction, $\phi$, was empirically determined by checking the phase behavior with the CLSM upon controlled dilution with solvent and extrapolating to the initial $\phi$. A sample typically consisted of $300 \mu \mathrm{L}$ in total with a particle concentration of $\phi=0.55$ containing $40 \mathrm{mM} \mathrm{Na} \mathrm{HPO}_{4} / \mathrm{NaH}_{2} \mathrm{PO}_{4}$ (Sigma Aldrich, 99\%) buffer of pH 7.8, $55 \mathrm{mM} \mathrm{NaCl}$ (Mag-Beta, 99\%) and $40 \mu \mathrm{M}$ Rhodamine-B (Sigma, 95\%). The final mixtures were prepared by mixing concentrated suspensions of PNIPAm and PNIPAm-AAc particles (typically 1:20) with a vortex.

\subsubsection{Confocal laser scanning microscopy}

CLSM measurements of the mixtures were done in sample cups prepared from Chromacol 2 -SV vials and round $22 \mathrm{~mm}$ Menzel Gläser \#1 microscope cover slips. The bottoms of the vials were removed and the cover slips were glued to the bottom using Bison Kombi Snel ${ }^{\oplus}$ two component epoxy glue.

Thermo-CLSM measurement cells used for the separate particle systems and mixtures were 
prepared using Menzel Gläser 24 x $50 \mathrm{~mm} \# 1$ and Menzel Gläser 20 x 20 mm \#1 microscope cover slips. The small slides were glued in between two larger slides at the side forming a rectangular sample cell. The PNIPAm mixture was placed inside the cell by capillarity force and the top and the bottom of the cell were sealed with glue to prevent solvent evaporation during the measurement.

Thin 2D cross-sections of the samples were imaged with a Nikon Eclipse TE2000U inverted microscope equipped with a Nikon C1 confocal scanning head and oil-immersion lens (100x Plan Apochromat, NA 1.4, Nikon). The lasers used for illumination were an air cooled Ar-ion laser (488 nm, Spectra Physics163C) for fluorescein and NBD-dyed particles and a HeNe laser (543.5nm, Melles Griot) for rhodamine dyed particles. Images were taken with a resolution of 512 x 512 pixels and a field of view between $70 \mu \mathrm{m} \times 70 \mu \mathrm{m}$ and $5 \mu \mathrm{m} \times 5 \mu \mathrm{m}$. The thermal behavior was studied using a Linkam TP 93 temperature controller and a Linkam THMS 600 microscope heating stage over a range of $22-42^{\circ} \mathrm{C}$.

\subsubsection{Data analysis}

Using purposely written routines in IDL (Interactive Data Language, Research Systems, Inc.; http://www.exelisvis.com (accessed July 2014)) the CLSM images of the crystals and defects were analyzed. The particle coordinates in 2D were obtained using algorithms described before ${ }^{[30,31]}$. From these coordinates, the local bond orientation order parameter $\Psi_{6}$, that is a measure for the local symmetry and bond orientation of the particles, was determined. It is defined as

$$
\Psi_{6}\left(r_{i}\right)=\frac{1}{n_{c}} \sum_{j=1}^{n_{c}} e^{6 i \theta\left(r_{i j}\right)}
$$
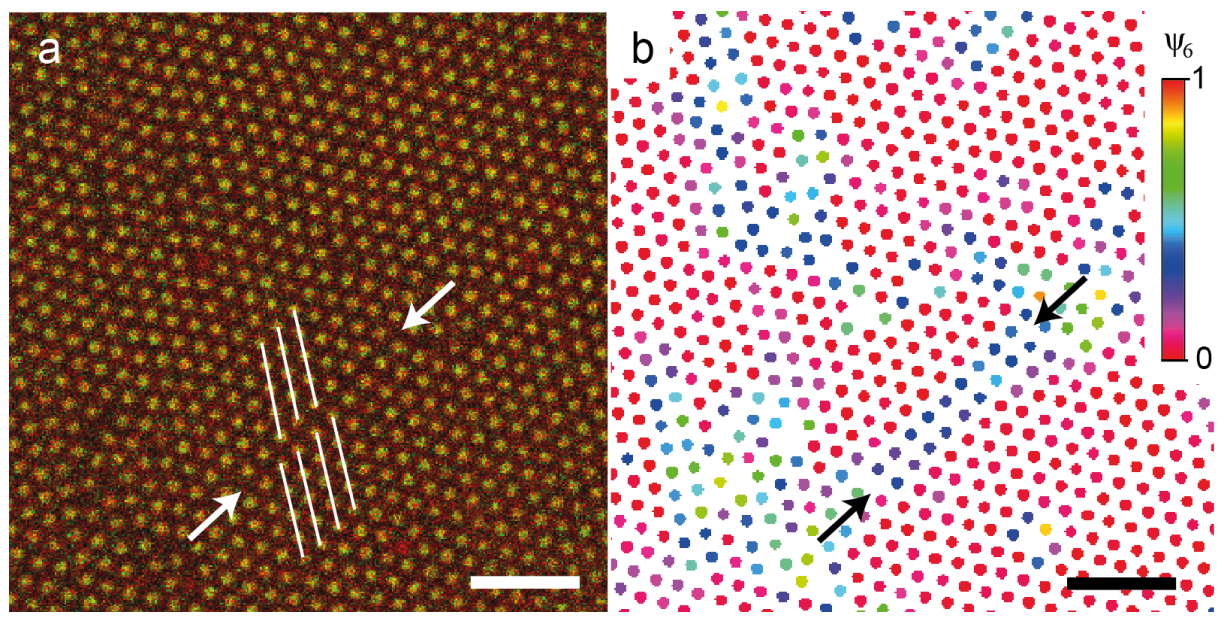

Figure 4.2. a) Typical CLSM image of a mixture of PNIPAm and PNIPAm-AAc in which a line-defect is present, indicated by white arrow. b) $\Psi_{6}$ analysis of the particle coordinates that identifies particles next to the line-defect (blue particles) due to their low $\Psi_{6}$ value. Scale bars are $5 \mu \mathrm{m}$. 
where the summation index $j$ runs over all, in total $n_{c}$ nearest neighbors of particle $i . \theta\left(r_{i j}\right)$ is the angle between the bond vector connecting particles $i$ and $j$ and an arbitrary fixed reference axis. Figure 4.2 shows a typical confocal microscopy image of a mixture of PNIPAm and PNIPAmAAc particles and the resulting $\left|\Psi_{6}\right|$ value of each particle. For a perfect hexagonal lattice $\left|\Psi_{6}\right|=1$, while lower $\left|\Psi_{6}\right|$ values correspond to defective regions. An in-plane dislocation, or commonly referred to as a line-defect ${ }^{[32]}$, indicated by the arrows and lines, can be distinguished by two rows of particles with a low $\left|\Psi_{6}\right|$ value (blue particles).

\subsection{Results \& Discussion}

\subsection{PNIPAm-AP \& silica}

The goal of this study is to develop a system of fluorescent PNIPAm particles embedded into a crystal of non-thermosensitive colloidal spheres and to study the defect dynamics via single particle tracking with CSLM. For CSLM studies the PNIPAm particles have to be fluorescent with a size of $R \sim 500 \mathrm{~nm}$. The first fluorescent PNIPAm particles were prepared by adding to the reaction mixture a fluorescent dye that is covalently attached a polymerizable group that can be incorporated into the polymer network. This was done by reacting fluorescein isothiocyanate with an excess of 3-amino-propene in a basic environment. Before addition to the reaction mixture the excess of 3-amino-propene was removed via evaporation.

This synthesis procedure resulted in monodisperse fluorescent particles of which we selected PNIPAm-AP due to its largest size. A typical confocal image of is shown in Figure 4.3.a. Although we found that the average particle size of PNIPAm-AP was smaller, $<R>=270 \mathrm{~nm}$, than the required size, preliminary mixing studies were performed with silica particles with $R=550 \mathrm{~nm}$. The silica particles were labeled with a rhodamine dye, of which the excitation
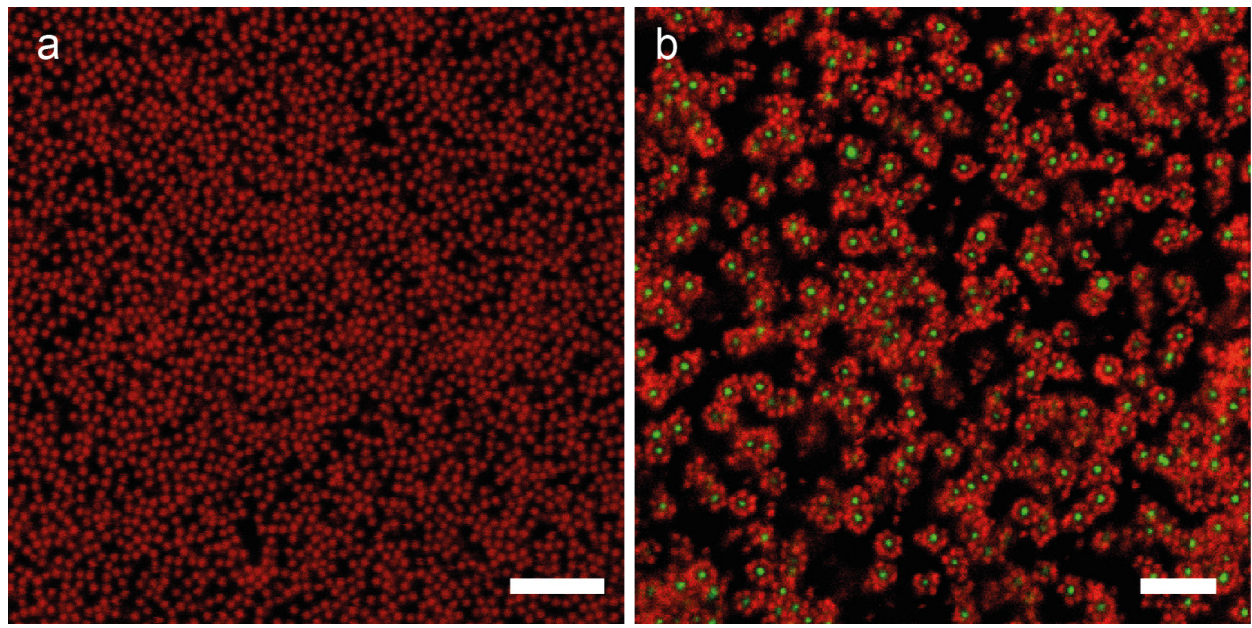

Figure 4.3. Typical CLSM image of a) Fluorescein labelled PNIPAm-AP particles and b) system I: PNIPAm-AP particles (red) mixed with Rhodamine-B labelled silica particles. Scale bars are $5 \mu \mathrm{m}$. 
and emission wavelength is significantly different than that of fluorescein, which allows us to distinguish the two particles in the mixtures.

Unfortunately, the mixtures we obtained did not show PNIPAm particles embedded into a colloidal crystal of silica particles, but rather the formation of clusters of silica particles surrounded by PNIPAm-AP particles. A typical CSLM image is shown in Figure 4.3.b. This behaviour can be explained by attractive interactions between the PNIPAm polymer chains and the silica particle surface. Therefore, the zeta potential, $\zeta$, was checked of both particles with for PNIPAm-AP $\zeta=-12.7 \mathrm{mV}$ and for the silica particles $\zeta=-41.4 \mathrm{mV}$. Although, both particles have negative potentials, the low zeta potential of the PNIPAm-AP particles indicates that positive charges could be present. This positive charge might be a result of the incorporation of 3-amino-propene monomers which were probably not completely removed from the dye mixture. In addition, positive charges might also be present on the silica particles by the incorporation of a rhodamine dye. The fact that 'flowers' are formed is the result of an excess of PNIPAm-AP particles that was added to prevent the expected fractionation by sedimentation. The PNIPAm density is much lower than that of Stöber silica, $\rho_{\mathrm{PNIPAm}}=1.05 \mathrm{~g} / \mathrm{cm}^{3}$ and $\rho_{\mathrm{SiO} 2}=$ $1.5 \mathrm{~g} / \mathrm{cm}^{3}$ and hence the silica particles were expected to fully sediment while the PNIPAm particles would remain in solution. Clearly, fractionation did not occur. Besides the unwanted structures, the depth of focus that could be achieved in the mixtures was very low. This can be explained by the different in refractive index of the particles. The PNIPAm particles are swollen with water and hence they are refractive index matched with the solvent. The silica particles, however, are not and will cause light scattering.

\subsubsection{PNIPAm \& PNIPAm-AAc}

These first experiments showed that another synthesis route should be used for the fluorescent labeling of the PNIPAm particles to prevent positive charges and that another nonthermosensitve particle system should be used for the mixtures, one that provides a similar inter-particle interaction, the same refractive index as water and has a similar density.

The system that fulfills these requirements is that of PNIPAm copolymerized with acrylic acid, PNIPAm-AAc. At high $\mathrm{pH}$ the acidic groups are charged and cause additional repulsion in the polymer network that prevents collapse of the network at high temperatures and moves the LCST towards higher temperatures ${ }^{[29]}$. Furthermore, when the PNIPAm-AAc particles are mixed with PNIPAm particles the inter-particle interactions will remain relatively the same. The PNIPAmAAc particles that were used were fluorescently labeled with an NBD dye ${ }^{[16]}$. To distinguish the PNIPAm and PNIPAm-AAc particles in a mixture the PNIPAm particles had to be labeled with Rhodamine dye. Unfortunately, the fluorescent labeling of PNIPAm is challenging and labeling of PNIPAm particles with a rhodamine dye in the network unsuccessful ${ }^{[33]}$. Therefore, we chose to stain the PNIPAm particles by adding rhodamine-B directly to the solution, where it was found to locate inside the particle network.

The thermosensitive behavior of both PNIPAm particles was first determined with SLS. Figure 4.4 shows the radius of gyration $R_{g}$ for PNIPAm-AAc and PNIPAm particles as determined 


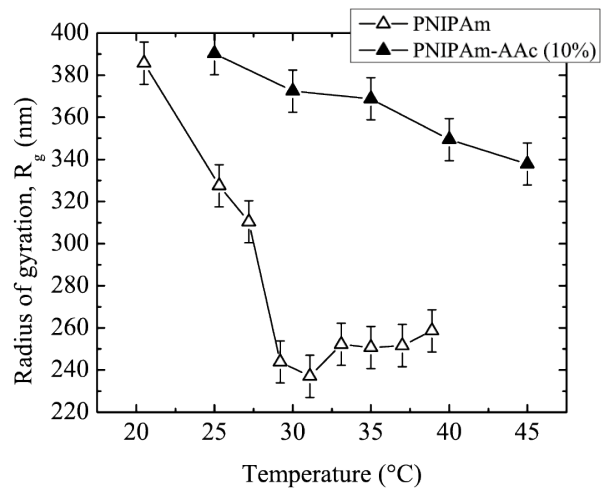

Figure 4.4. $R_{g}$ of the PNIPAm and PNIPAm-AAc particles as a function of temperature determined via SLS.

from SLS measurements at pH 7.8 and $50 \mathrm{mM} \mathrm{NaCl}$. At RT very similar particle sizes are obtained, $R_{g}=390 \mathrm{~nm}$ and $386 \mathrm{~nm}$, respectively. Above $30^{\circ} \mathrm{C}$ the PNIPAm particles show a size decrease of $\Delta R \approx 140 \mathrm{~nm}$ while for the PNIPAm-AAc particles only a slight decrease of $\Delta R \approx 20$ $\mathrm{nm}$ is observed. Clearly, the acrylic acid molecules present in PNIPAm-AAc particle influence the LCST and repress the particle decrease in size, making their combination perfect for the proposed defect studies.

CLSM temperature studies, both heating and cooling, were done of both separate particles systems to check the temperature response at high $\phi$. Figure 4.5 shows the effect of a fast temperature increase of $0,167^{\circ} \mathrm{C} / \mathrm{s}$ and the sequential CLSM images from $25^{\circ} \mathrm{C}$ to $40^{\circ} \mathrm{C}$ of
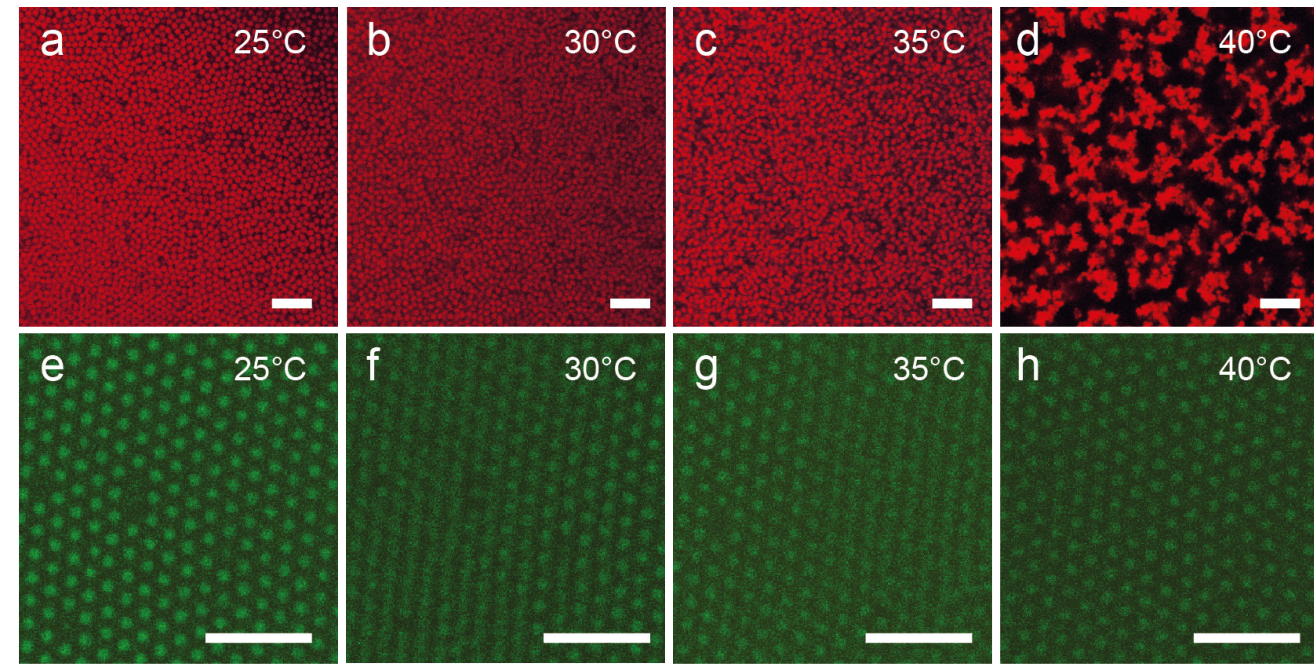

Figure 4.5. CLSM images over a temperature range of $25-40^{\circ} \mathrm{C}$ for (a-d) the PNIPAm particles showing their VPT and (e-h) PNIPAm-AAc particles that retain their size. Scale bars are $5 \mu \mathrm{m}$. 
concentrated dispersions of both particles. The PNIPAm particles (Fig.4.5.a-d) show the expected thermo-sensitive behavior with the transition from a glass phase at $25^{\circ} \mathrm{C}$ to a very mobile fluid phase between $30-35^{\circ} \mathrm{C}$. At $40{ }^{\circ} \mathrm{C}$ clustering and the formation of large aggregates was observed, indicating that the steric stabilization of the particle surface has been replaced by a hydrophobic attraction. The PNIPAm-AAc particles show their expected behavior with the presence of a crystal phase over the full temperature range (Fig.4.5.e-h) and clearly do not change their size.

Several problems were encountered during the CLSM experiments. Firstly, imaging of the same crystal plane in the structures was difficult over time as the focal plane of the microscope changed with temperature. This is the reason why in Figure.4.5.f and 4.5.g the crystal planes are partly out of focus. This problem was caused by the handmade thermo-CLSM cells, in which at high temperature, glue expansion and leakage of the sample fluid was observed. Secondly, a fast temperature change rate of at least $\pm 0.1^{\circ} \mathrm{C} / \mathrm{s}$ was needed. With slower rates the VPT of the PNIPAm particles was much less pronounced; the particles retained their size and their steric stabilization. Thirdly, dye bleaching of both dyes occurred, with a faster rate for the NBD dye, and samples had to be imaged on the day of preparation, with a low laser power and a low frame rate, complicating the measurements. Lastly, the presence of the rhodamine-B dye in solution resulted in a number of undesired effects. The dye increased the background noise in the CLSM images and in the presence of the dye PNIPAm particles were found to stick to the glass wall.

\subsubsection{Temperature studies of mixtures}

In spite of all the above mentioned problems, mixtures could be successfully prepared of the PNIPAm and PNIPAm-AAc particles. Figure 4.6.a shows an CLSM image of a mixture of 95\% PNIPAm-AAc particles and 5\% PNIPAm particles at $35^{\circ} \mathrm{C}$. It is clear that the PNIPAm particles are successfully incorporated into a PNIPAm-AAc crystal and are located at crystal lattice positions. Due to the preference of the rhodamine-B dye in solution the PNIPAm-AAc particles appear orange, but with proper detector channel selection they can still be distinguished from the PNIPAm particles (Fig.4.6.b-c). Furthermore, at this high temperature the PNIPAm particles do not show the size decrease expected from the SLS measurements. However, they were observed to wiggle around their lattice positions, while the PNIPAm-AAc lattice remained stationary. This indicates that although the temperature response of the PNIPAm particles is suppressed a small size change can be achieved and the particles are still stable.

The PNIPAm and PNIPAm-AAC mixtures were imaged with the CSLM over a temperature range to study if the shrinking and swelling of the PNIPAm particles would have an effect on the PNIPAm-AAc crystal and would cause defect movement. The measurements were performed over temperature range of $22^{\circ} \mathrm{C}$ to $40^{\circ} \mathrm{C}$ with a temperature rate between $0.05-0.17{ }^{\circ} \mathrm{C} / \mathrm{s}$ and by carefully keeping the desired (x,y) crystal plane in focus manually. A low imaging rate of 1.7 $\mathrm{s}$ /frame was used to prevent dye bleaching during the time needed for a full temperature scan.

In contrast to our expectations, upon an increase in temperature structural changes or defect formation were not observed in the mixed crystals. However, upon a decrease in temperature 


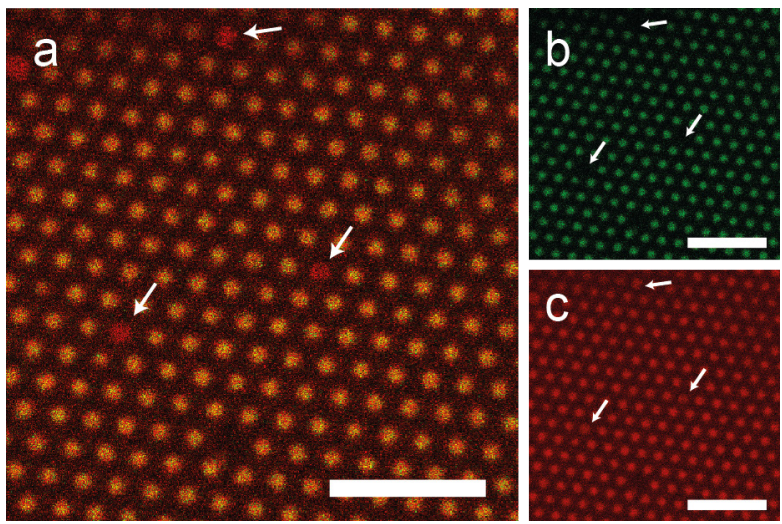

Figure 4.6. a) CLSM image of a PNIPAm-AAc crystal (green/orange) incorporated with PNIPAm particles (red) at several lattice positions, indicated with the white arrows. b) selection of green detector channel showing the location of only the PNIPAm-AAc particles. c) selection of red detector channel showing location of all particles. Scale bars are $5 \mu \mathrm{m}$.

particle and defect movements did occur, surprisingly even when the initial temperature was below the LCST. Several defects, such as dislocations, vacancies and interstitials as well as their diffusion, were observed in the mixed crystals. Interestingly, also the displacement of complete crystal plane areas, accompanied by the appearance and disappearance of line-defects, were observed.

Figure 4.7 shows three frames of a cooling series from $30^{\circ} \mathrm{C}$ to $22^{\circ} \mathrm{C}$ with a cooling rate of $-0.05^{\circ} \mathrm{C} / \mathrm{s}$. The frames were taken at $25.6^{\circ} \mathrm{C}, 25.5^{\circ} \mathrm{C}$ and $25.4^{\circ} \mathrm{C}$, respectively and show different defects including the formation of a line-defect. The defects and their movement are indicated by different symbols in Figure 4.6.a-c. The white arrows indicate the positions of PNIPAm particles, the red dashed line represents the boundaries of the in-plane movement of the crystal areas along the red arrows, the white circles indicate vacancies and the stars indicate dislocations in the perfect hexagonal arrangement. Interestingly, the defect movements occurred in a specific order. First the triangular areas were shifted up and down while the first dislocation point moved up and a second one was created. This was followed by the movement of the second area resulting in rearrangement of the second distorted point and creation of a third. At the same time one of the vacancies disappears by the movement of an additional particle from a layer below or above, that indicates that the defect diffusion occurs in $3 \mathrm{D}$. Clearly, the structure is undergoing rearrangements due to the swelling of the PNIPAm particles.

Careful analysis of each image and the $\Psi_{6}$ evaluation (Fig. 4.7.d-f) shows that an additional row of particles in hexagonally ordered crystal plane, an edge-dislocation, ends at the dislocation. Interestingly, this is also where the defect movement starts that in the second and third frame results in rearrangement of the hexagonal order in the layer and the formation of a line-defect. The line-defect does not fully extend in the layer, as is illustrated by the black lines in Figure. 4.7.f, that remain parallel above and below the defect. 

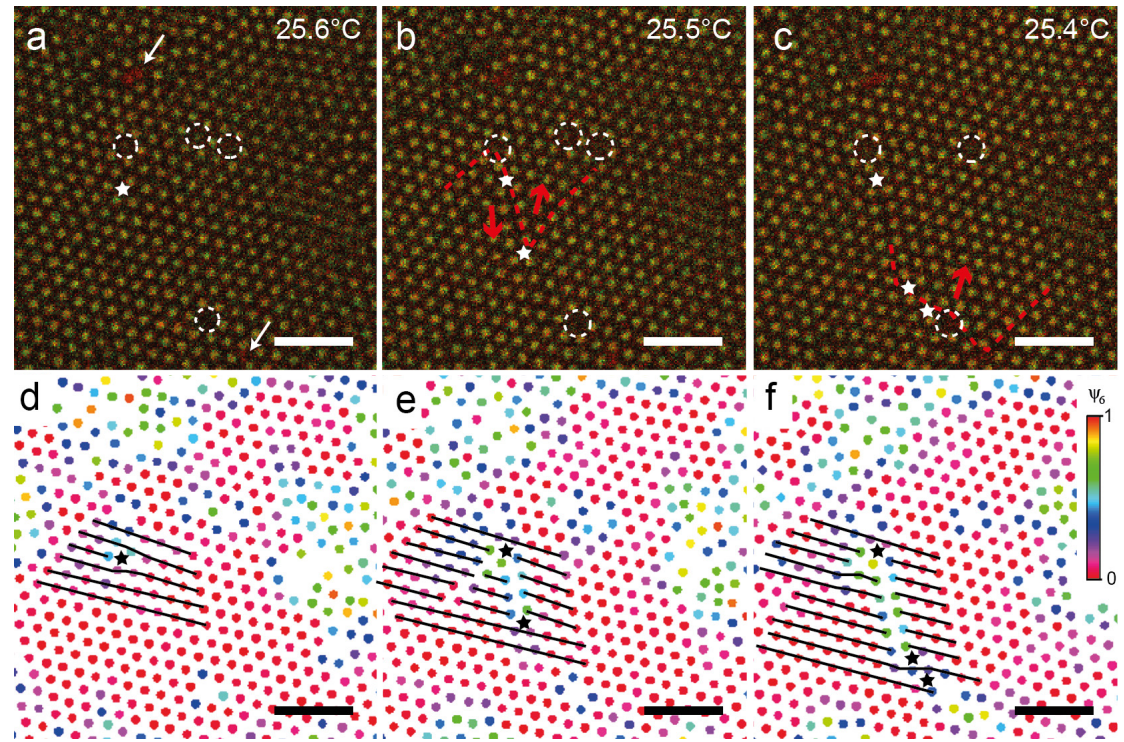

Figure 4.7. a-c) Three subsequent CLSM images of the PNIPAm-AAc crystal doped with PNIPAm particles upon cooling from $30-22{ }^{\circ} \mathrm{C}$ showing defect movement. White arrows indicate PNIPAm particles, circles indicate vacancies, stars indicate a disordered point and red dashed line define border of crystal plane areas that have moved in the direction of the red arrows. d-f) $\Psi_{6}$ analysis of the images showing the overall hexagonal order in the layer. Due to the movement of the crystal plane areas a line-defect is formed, as indicated by the rows of blue particles. These end in the two point distortions. $s$ are $5 \mu \mathrm{m}$.
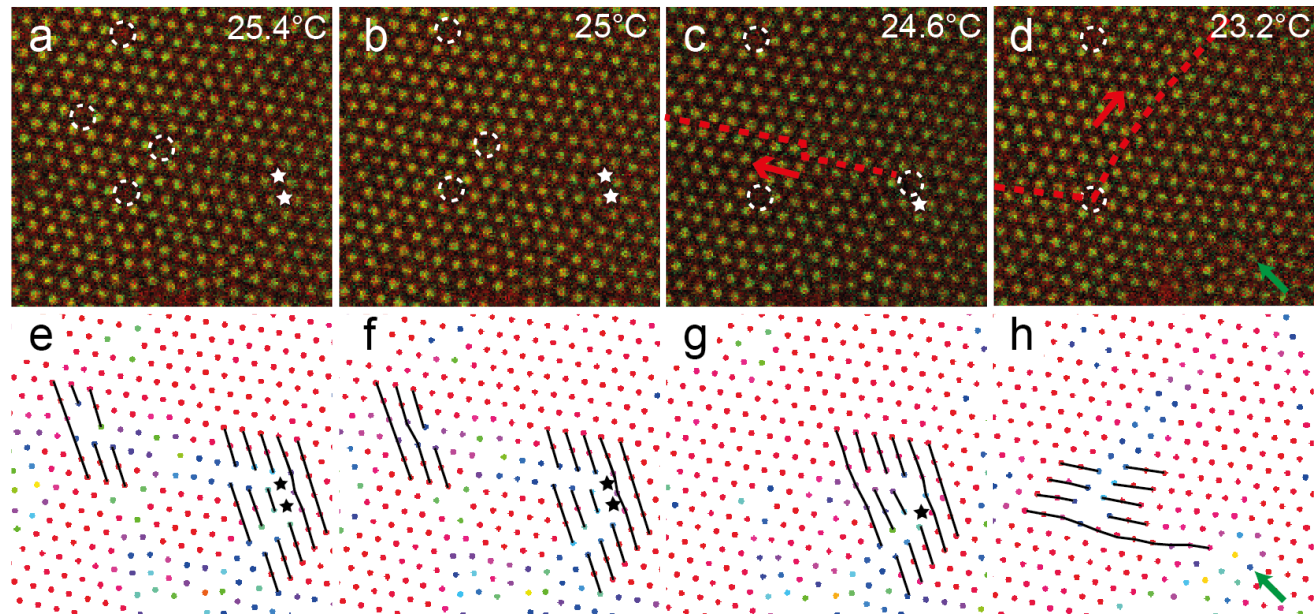

f $\because \because \cdots \because \because \because \because \because \because \because \because g$

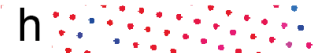

Figure 4.8. a-d) Four CLSM images of a cooling series from $30-22{ }^{\circ} \mathrm{C}$ of the PNIPAm-AAc crystal doped with PNIPAm particles. d-f) $\Psi_{6}$ analysis of the images showing the overall hexagonal order in the layer. A line-defect disappears and another is formed. Scale bar are $5 \mu \mathrm{m}$. 
Figure 4.8 shows four frames of another cooling series, where the temperature was also decreased from $30^{\circ} \mathrm{C}$ to $22^{\circ} \mathrm{C}$ and the frames a,b,c and $\mathrm{d}$ are respectively at $25.4^{\circ} \mathrm{C}, 25^{\circ} \mathrm{C}, 24.6^{\circ} \mathrm{C}$ and $23.2^{\circ} \mathrm{C}$. The line-defect, running from the left vacancy towards the two dislocations on the right, was observed from the start of the measurement. It remained stable until $25.4^{\circ} \mathrm{C}$ after which it disappears in two steps. A particle is first added at the position of the first vacancy followed by the addition of a second particle and an area movement to the left. This leaves the line defect corner and only one distorted point, as the top one has been transformed into a full vacancy. After some additional movement in the crystal a line-defect is formed that starts at the remaining vacancy in the bottom part of the crystal, and seems to be stabilized by the remainder of the line-defect of Figure 4.8.c\&g.

These measurements show that the goal of inducing vacancies and interstitials (see Fig.4.1.) is not completely achieved with the mixed PNIPAm-AAC + PNIPAm system. However, this mixed system does show interesting defect movement upon a temperature decrease that must be related to the VPT of the PNIPAm particles, as for pure PNIPAm-AAC crystals defect movements were not observed. Figure 4.9 shows a schematic representation of the achieved effect with the PNIPAm particles in a PNIPAm-AAc crystal. The PNIPAm particles were found to be located at both lattice and interstitial positions as single particles as well as in clusters. Upon a temperature decrease the particles swell and induce stress in the crystal that leads to the movement of particles and defects. Evidently, defect diffusion can be studied with a mixed system of PNIPAm and PNIPAm-AAc.

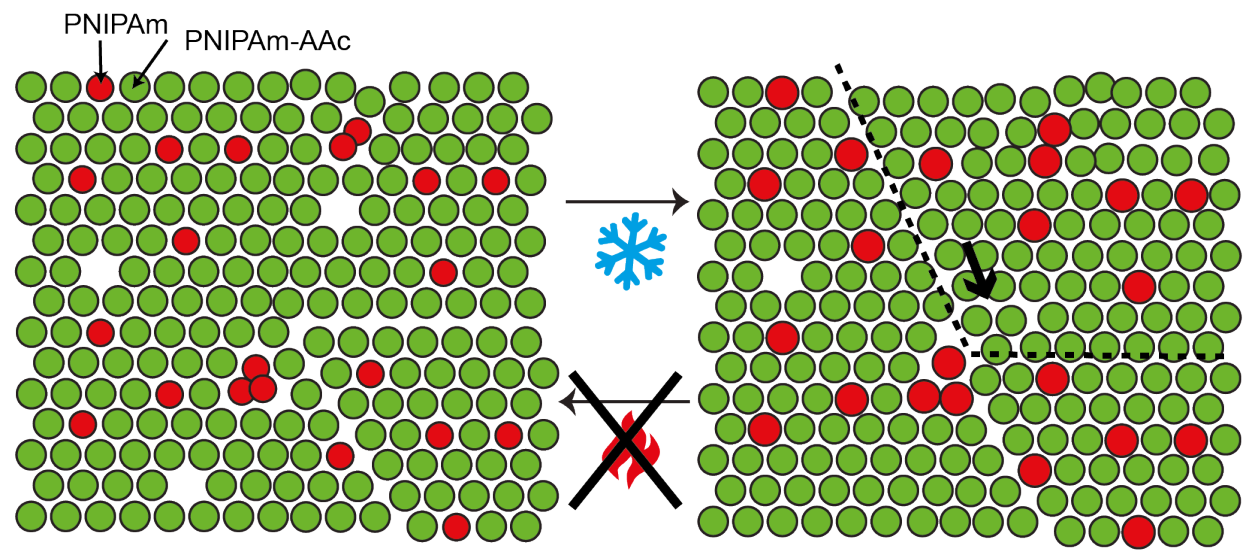

Figure 4.9. Schematic representation of the defect movement observed in a mixed system of PNIPAm and PNIPAm-AAC particles. Upon a temperature decrease the PNIPAm particles that occupy several places in a crystal plane, will increase in size slightly causing the movement of defects and in-plane displacements of certain areas. Here, the line-defect is elongated by a shift of the top part downward. 


\subsection{Conclusion}

The aim of this study was the preparation of thermosensitive colloidal crystal defects by mixing thermoresponsive PNIPAm particles into a crystal of nonresponsive particles for the study of defect formation and diffusion. The first attempts were made with mixtures of PNIPAm-AP particles and silica spheres and showed that the incorporation of PNIPAm particles into this non-PNIPAm system did not result in the desired system. Flower-like clusters were obtained due to the presence of attractive inter-particle forces.

Mixtures of PNIPAm and PNIPAm-AAc, that have similar inter-particle interactions, were found to provide stable mixtures. Due to its reduced thermosensitive behavior, the PNIPAmAAc particles were able to form the colloidal crystal matrix. Temperature studies revealed that only upon a temperature decrease the formation of several types of defects can be induced. The appearance and disappearance of line-defects indicates the presence of structural reorganization to accompany the additional pressure in the system. Addition of particles to the imaged layer also indicates that the defect movement occurs in $3 \mathrm{D}$. However, due to numerous problems that arose during the measurements, the low data quality and the fact that only a single plane was imaged, makes it difficult to elucidate the origin of the defects in this system.

This research does show that the in situ study of colloidal crystal defects can be achieved by introducing thermosensitive PNIPAm particles as 'defects' into a non-thermo responsive colloidal crystal and that with different fluorescent labeling the defect particles could be traced. Even though the systems used here were clearly not ideal yet, the results indicate that by employing PNIPAm particles we are on the right track for the study of $3 \mathrm{D}$ defect dynamics in colloidal crystals.

\subsection{Outlook}

The incorporation of a fluorescent dye into the PNIPAm network was the biggest challenge in achieving a system suitable for real space and real time defect dynamics studies with an CLSM. Clearly, the addition of rhodamine-B dye directly into the solvent adds charges to the system that result in undesired interactions. We would, therefore, strongly recommend against the use of it for further studies.

The recent synthesis of fluorescent PNIPAm particles done by Sprakel et al. is very promising $^{[34]}$. They showed the incorporation of commercially available dyes into a polystyrene core that is surrounded by a colorless PNIPAm-AAc shell. This makes the particles very stable against bleaching and allows for accurate determination of the particle centers. Sprakel et al. used these particles for studies of large impurities in a crystal and showed the effect of the number density on the crystal structures. Employing these core-shell particles with PNIPAm and PNIPAm-AAc shells in the experiments described above, could provide the data quality over time and in $3 \mathrm{D}$ that is desired. 


\section{Acknowledgements}

This work is the result of collaborations with many people. First and foremost Jan Hilhorst is thanked for many useful discussions, experimental support and for providing the PNIPAm-AAc particles. Daan Verwijmeren is thanked for his obtained insight into the PNIPAm synthesis and Cesare Benedetti is thanked for performing the experiments and acquiring the data for Figures 4.4-4.8.

\section{References}

1. H. G. Bueren, Imperfections in crystals, North-Holland Publishing Company, Amsterdam, 1961.

2. D. Hull and D. J. Bacon, Introduction to dislocations., Elsevier Ltd., 2001.

3. R. S. Muller, I. K. Theodore and M. Chan, Device electronics for integrated circuits., Wiley, 2002.

4. G. I. Taylor, Proc. R. Soc. London, Ser. A, 1934, 145, 362-387.

5. Y. N. Osetsky, D. J. Bacon, B. N. Singh and B. Wirth, J. Nucl. Mater., 2002, 307, 852-861.

6. Y. N. Osetsky, A. Serra, B. N. Singh and S. I. Golubov, Philos. Mag. A, 2000, 80, 2131-2157.

7. W. Bollman, Physical Review, 1956, 103, 1588-1589.

8. J. W. Menter, Proc. R. Soc. London, Ser. A, 1956, 236, 119.

9. P. B. Hirsch, R. W. Horne and M. J. Whelan, Philosophical Magazine, 1956, 1, 677.

10. K. Arakawa, K. Ono, M. Isshiki, K. Mimura, M. Uchikoshi and H. Mori, Science, 2007, 318, 956-959.

11. Y. Matsukawa and S. J. Zinkle, Science, 2007, 318, 959-962.

12. J. Perrin, Annales De Chimie Et De Physique, 1909, 18, 5-114.

13. U. Gasser, E. R. Weeks, A. Schofield, P. N. Pusey and D. A. Weitz, Science, 2001, 292, 258-262.

14. P. G. Bolhuis, D. Frenkel, S. C. Mau and D. A. Huse, Nature, 1997, 388, 235-236.

15. J. Brijitta, B. V. R. Tata, R. G. Joshi and T. Kaliyappan, J. Chem. Phys., 2009, 131, 074904.

16. J. Hilhorst and A. V. Petukhov, Phys. Rev. Lett., 2011, 107, 095501.

17. A. Pertsinidis and X. Ling, Nature, 2001, 413, 147-150.

18. A. Pertsinidis and X. S. Ling, New Journal of Physics, 2005, 413, 147-150.

19. C. Eisenmann, U. Gasser, P. Keim, G. Maret and H. H. von Grunberg, Phys. Rev. Lett., 2005, 95, 185502.

20. A. Libal, C. Reichhardt and C. J. O. Reichhardt, Phys. Rev. E, 2007, 75, 011403.

21. W. Lechner and C. Dellago, Soft Matter, 2009, 5, 2752-2758.

22. L. C. DaSilva, L. Candido, G. -Q. Hai and O. N. Oliveira Jr., Appl. Phys. Lett., 2011, 99, 031904.

23. S. van Teeffelen, C. V. Achim and H. Loewen, Phys. Rev. E, 2013, 87, 022306.

24. W. Lechner, D. Polster, G. Maret, P. Keim and C. Dellago, Phys. Rev. E, 2013, 88, 060402.

25. R. Pelton, Adv. Colloid Interface Sci., 2000, 85, 1-33.

26. R. Pelton, J. Colloid Interface Sci., 2010, 348, 673-674.

27. A. M. Alsayed, M. F. Islam, J. Zhang, P. J. Collings and A. G. Yodh, Science, 2005, 309, 1207-1210.

28. Y. Peng, Z. Wang, A. M. Alsayed, A. G. Yodh and Y. Han, Phys. Rev. Lett., 2010, 104, 205703.

29. M. Karg, I. Pastoriza-Santos, B. Rodriguez-Gonzalez, R. von Klitzing, S. Wellert and T. Hellweg, Langmuir, 2008, 24, 6300-6306.

30. J. C. Crocker and D. G. Grier, J. Colloid Interface Sci., 1996, 179, 298-310.

31. V. W. A. de Villeneuve, R. P. A. Dullens, D. G. A. L. Aarts, E. Groeneveld, J. H. Scherff, W. K. Kegel and H. N. W. Lekkerkerker, Science, 2005, 309, 1231-1233.

32. J. M. Meijer, V. W. A. de Villeneuve and A. V. Petukhov, Langmuir, 2007, 23, 3554-3560.

33. D. L. M. Verwijmeren, Synthesis of Fluorescent PNIPAm, Master Thesis, Utrecht University, 2012.

34. R. Higler, J. Appel and J. Sprakel, Soft Matter, 2013, 9, 5372-5379. 


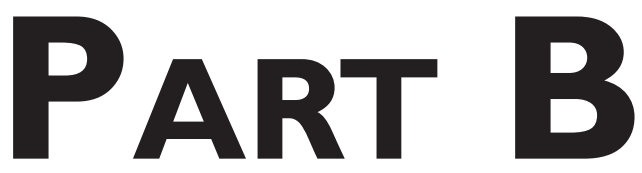

$\bullet$

$\bullet$

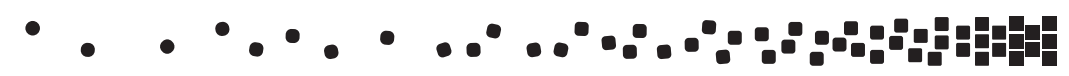

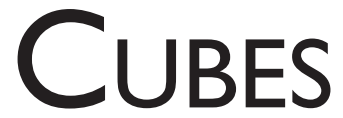



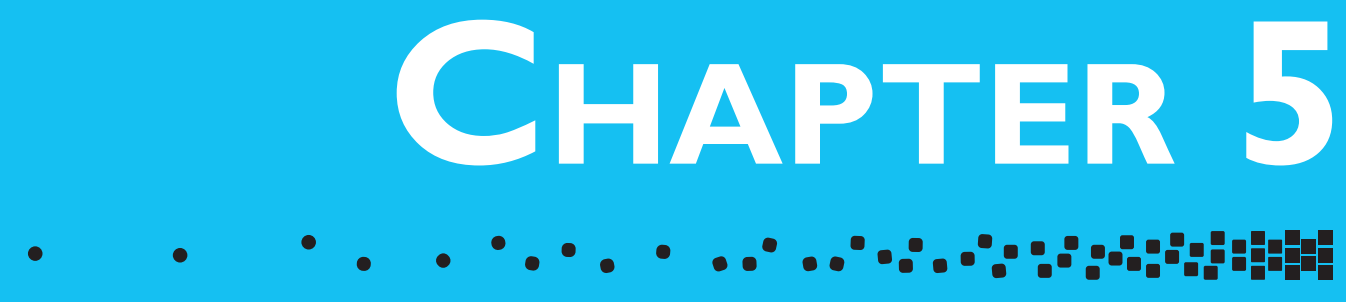

\section{Preparation and Characterization of Colloidal Cubes}

Abstract

This chapter describes the synthesis of the three different types of colloidal cubes: hematite cubes, silica coated hematite cubes and hollow silica cubes, used for the experiments reported in this thesis. The way to reproducibly prepare the hematite cubes is describes as well as how to tune the particle size. In addition, the silica coating is presented that after removal of the hematite core allows us to obtain the hollow silica cubes. An overview is given of the size, shape and interactions of the various cubes that were prepared.These properties are relevant for the structure formation studies in Chapters 6 to 9. 


\section{I. Introduction}

Development in synthesis has led to systematic preparation of colloidal particles with a large variety of shapes, such as dumbbells $s^{[1,2]}$, ellipsoids ${ }^{[3]}$, cubes $^{[4-11]}$, polyhedral ${ }^{[10]}$, octapods ${ }^{[12]}$, and many more ${ }^{[13,14]}$. The experimental realization of these anisotropic shapes allow for explorations of the geometrical effects on their dense structures, which have been intensively investigated via theory and simulation ${ }^{[15,16]}$.

Colloidal particles with a cube shape are of specific interest, because cubic building blocks allow the formation of essentially space filling structures and of rare crystal symmetries such as the simple cubic (SC) lattice ${ }^{[8,17]}$. Furthermore, colloidal cubes are suitable experimental pendants of theories and simulations of cube fluids, which have shown that the cubic shape impacts their phase behavior and results in new crystal symmetries ${ }^{[17-22]}$.

Colloidal cubes can be synthesized that have different sizes and shapes and are composed of different materials. Single crystalline colloidal cubes can be prepared from many inorganic materials, such as ironoxide ${ }^{[5,23,24]}$, gold ${ }^{[6]}$, silver ${ }^{[7,10]}$, palladium ${ }^{[8]}$ and $\operatorname{copper}^{[11]}$. The cubic shape is obtained by the use of specific additives that cause different growth rates of the crystal facets ${ }^{[25]}$. However, this growth mechanism often promotes the formation of truncated cubes. In addition, the particle sizes are limited to approximately 5-100 nm, with an exception of $300 \mathrm{~nm}$ for silver cubes ${ }^{[10]}$. Colloidal cubes with a polycrystalline interior can also be synthesized as was shown for sodium magnesium fluoride ${ }^{[26]}$ as well as hematite ${ }^{[4,9]}$. The size of these polycrystalline cubes is relatively large $(\sim 1 \mu \mathrm{m})$ compared to the single crystalline cubes. However, their growth mechanisms cause the cubes to possess either high polydispersity or a high degree of roundness of the cube corners.

In this thesis we focus on the micron-sized colloidal cubes of hematite and employ them in several crystallization studies. Due of their large size, these hematite cubes can be visualized in real-space with optical microscopy, in contrast to the smaller single crystalline cubes referred to above. Furthermore, it has also been shown that the hematite cubes can be used as templates for hollow silica cubes ${ }^{[3,9]}$. In this way, the interparticle interactions can be tuned from the attractive interactions due to a hematite core to the repulsion between hollow, charged silica cubes. Moreover, their rounded cube corners make them colloidal realizations of superball shaped particles, which have been extensively studied by simulations ${ }^{[19-22]}$.

In this chapter the synthesis of three types of colloidal cubes, namely hematite cubes, silica coated hematite cubes and hollow silica cubes are presented that are employed in this part of the thesis. In addition, several cube properties are investigated and we discuss their effect on the cube interactions, that play a role during the assembly of crystalline structures. 


\subsection{Experimental}

\subsection{I.Hematite cubes synthesis}

The colloidal hematite cubes are synthesized according to the gel-sol method first described by Sugimoto et al. ${ }^{[4]}$ and further studies have led to well-documented synthesis procedures ${ }^{[3,23,27,28]}$. The obtained hematite cubes have a well-defined size and shape with a low polydispersity.

A typical synthesis for the hematite cubes in this thesis started with of the preparation of a yellow $2 \mathrm{M} \mathrm{FeCl}_{3}$ solution by dissolving $54 \mathrm{~g}$ of fresh $\mathrm{FeCl}_{3} \cdot 6 \mathrm{H}_{2} \mathrm{O}$ (Sigma Aldrich) in $100 \mathrm{~mL}$ demineralized water in a $250 \mathrm{~mL}$ Pyrex bottle. Demineralized water was obtained from a Millipore water system and was used for all experiments. To this $\mathrm{FeCl}_{3}$ solution $90 \mathrm{~mL}$ of $6 \mathrm{M}$ $\mathrm{NaOH}$ (Merck) and $10 \mathrm{~mL}$ water were added rapidly (20s) under vigorous magnetic stirring. The $\mathrm{NaOH}$ addition causes the formation of a condensed ferric hydroxide gel containing small particles of iron(III)hydroxide, $\mathrm{Fe}(\mathrm{OH})_{3}$, manifested by the dark brown (almost black) color of the gel. After stirring $10 \mathrm{~min}$ the mixture was aged at $100^{\circ} \mathrm{C}$ in an oven, during which time the $\mathrm{Fe}(\mathrm{OH})_{3}$ transforms into akaganeite, $\beta-\mathrm{FeO}(\mathrm{OH})$, needles that finally transform into hematite, $\alpha-\mathrm{Fe}_{2} \mathrm{O}_{3}$, in the form of particles with a cubic shape. The hematite particles settle into a dark red colored sediment leaving behind a clear yellow supernatant. Occasionally the synthesis product was a mixture of needles and small hematite clusters instead of cubes, due to an incomplete conversion of the akaganeite needles into hematite. The hematite cubes sediment was washed by centrifugation and redispersed in water repeatedly. Due to the high salt concentration and low $\mathrm{pH}$, we typically used $1 \mathrm{~L}$ centrifuge tubes that were placed in an Avanti ${ }^{\mathrm{TM}} \mathrm{J}-20 \mathrm{XP}$, Beckman Coulter centrifuge providing an acceleration of $500 \mathrm{~g}$.

The number and quality of the precursor particles for the hematite cubes was found to be related to the details of the mixing procedure of the $\mathrm{FeCl}_{3}$ and $\mathrm{NaOH}$ solutions ${ }^{[28]}$. The addition speed of the $\mathrm{NaOH}$, the stirring speed and the temperature of the solution all influence the final particle size and shape. Furthermore, the purity of the solutions may affect the obtained particle shape. For instance, the presence of $\mathrm{SO}_{4}^{2-}$ ions promotes the formation of spindles or even peanuts ${ }^{[23,29,30]}$. When all factors above are kept constant the cube size can be controlled by the excess concentration of $\mathrm{Fe}^{3+}$ with respect to the $\mathrm{OH}^{-}$concentration. Therefore, the use of fresh $\mathrm{FeCl}_{3} \cdot 6 \mathrm{H}_{2} \mathrm{O}$ is advised because $\mathrm{FeCl}_{3}$ is known to be hygroscopic. Typically an excess concentration of $0.020 \mathrm{~mol} \mathrm{Fe}^{3+}$ is present and small changes in the $\mathrm{NaOH}$ concentration can be used to tune of the cube edge length between $500-1500 \mathrm{~nm}$ although exact control over the edge length is impossible. Particles smaller than $500 \mathrm{~nm}$ can also be obtained but these tend to have a diamond shape, while particles larger than $1500 \mathrm{~nm}$ tend to have curved faces.

\subsubsection{Silica coating of hematite cubes}

The hematite cubes are coated with amorphous silica using an adaption of the Stöber synthesis ${ }^{[9,31]}$. The hematite cubes are first functionalized by the adsorption of polyvinyl pyrolidone (PVP) on their surface. For the particles in this thesis this was typically achieved by dissolving $10 \mathrm{~g}$ PVP ( $\mathrm{Mw}=10.000$ or 40.000 , Aldrich) in $100 \mathrm{~mL}$ water and adding it to $100 \mathrm{~mL}$ $7 \mathrm{wt} \%$ cube dispersion. The adsorbed PVP stabilizes the hematite particles against aggregation 
and serves as an anchoring point for the forming silica ${ }^{[31]}$. After 16 hours the excess PVP was removed via centrifugation and the particles were redispersed in ethanol (100\%, Mercachem). The PVP functionalized cubes can be kept in ethanol for a few days but longer waiting times will lead to desorption of the PVP and inhomogeneous coating of the cubes. For the silica coating $83 \mathrm{~mL}$ of $6.2 \mathrm{wt} \%$ PVP coated hematite cubes dispersion, $782 \mathrm{~mL}$ ethanol $(100 \%$, Fisher Chemicals), $80 \mathrm{~mL}$ water were mixed in a $2 \mathrm{~L}$ round bottom flask. To this $7 \mathrm{~mL} 3 \mathrm{wt} \%$ tetramethyl ammonium hydroxide (TMAH, Sigma Aldrich) is added that elevates the $\mathrm{pH}$ which is needed to catalyze the hydrolysis of the silica precursor tetraethyl orthosilicate (TEOS). The mixture was mechanically stirred and sonication was applied, which prevents clustering of the cubes due to their magnetic attractions. The silica deposition was started by slow addition (one drop every four seconds) of a mixture of $16 \mathrm{~mL}$ tetraethylorthosilicate (TEOS, purum, >98\% Fluka) and $16 \mathrm{~mL}$ ethanol (p.a. Merck) using a peristaltic pump. By varying the amount of TEOS the thickness of the silica shell can be tuned. Usually the amount of TEOS was based on estimates of the cube surface area and a desired silica thickness of 20-150 nm. After the TEOS addition was completed, the mixture was stirred and sonicated for an additional 2 hours to complete the reaction. Next, 5g PVP in $100 \mathrm{~mL}$ ethanol is added to improve the stability of the cubes. After $16 \mathrm{~h}$ the reaction mixture was centrifuged to remove the excess of PVP and unreacted TEOS molecules and the obtained coated cubes were dispersed in ethanol.

\section{Fluorescent labeling}

A fluorescent dye can be added during the coating step and will be incorporated into the silica shell. This fluorescent labeling allows tracking of the particles with a laser scanning confocal microscope. For the fluorescent labelling a similar coating procedure was performed as described above, but with two coating steps separated by a washing procedure instead of one coating step. The first coating was typically performed with a dye solution that was prepared by mixing $6.20 \mathrm{mg}$ fluoresceine isothiocyanate, $0.80 \mathrm{~g} \mathrm{3-aminopropyl-triethoxysilane} \mathrm{and}$ $7.76 \mathrm{~g}$ ethanol resulting in a yellow/orange solution. In the first silica coating step $4 \mathrm{~mL}$ dye solution, $7.7 \mathrm{~mL}$ TEOS and $5 \mathrm{~mL}$ ethanol were added drop wise to the reaction mixture of PVP functionalized cubes, ethanol, water and TMAH. After completion of this coating step the dispersion was centrifuged and redispersed in a new reaction mixture. The second coating step was performed with a mixture of $8 \mathrm{~mL}$ TEOS mixed with $5 \mathrm{~mL}$ ethanol.

\subsubsection{Hollow silica cubes}

Hollow silica cubes can be prepared by removing the hematite core cubes from the silica shell, achieved by dispersing the silica coated hematite cubes in concentrated hydrochloric acid ( $\mathrm{HCl})$. In this thesis work typically $150 \mathrm{~mL} \mathrm{HCl}$ (37\%, Merck) was added to $150 \mathrm{~mL}$ of $5 \mathrm{wt} \%$ silica coated hematite cube dispersion in water and vigorously mixed, by shaking or magnetic stirring. The suspension was allowed to settle or was stirred for at least 16 hours but usually 24 hours during which the dispersion underwent a color change of dark red to yellow. It has to be noted that the silica shells are stable under shear, but prolonged magnetic stirring can 
damage the cubes and should be avoided. Repeated centrifugation and redispersion in water was performed until the $\mathrm{pH}$ of the supernatant was found to be in the range 5-7, corresponding to that of Millipore water. The obtained suspension of hollow silica cubes had a turbid, white appearance. If an orange suspension was obtained this indicated that not all hematite was removed from the silica shell and the $\mathrm{HCl}$ procedure was repeated. The obtained white cube dispersion was kept in ethanol for storage, as amorphous silica is known to slowly dissolve in water $^{[32]}$.

\subsubsection{Characterization}

Transmission electron microscopy (TEM) was performed with a Philips TECNAI 10 or TECNAI 12 microscope. A sample was prepared by drying a dilute drop of dispersion on a polymer coated copper grid.

Light microscopy measurements of the particle dispersions were obtained with a Nikon Eclipse Ti inverted microscope, equipped with a 100x Nikon oil objective and a Lumenera INFINITYX camera. Samples were prepared by placing the cube dispersions into flat VitroCom optical capillaries $(50 \times 2 \times 0.1 \mathrm{~mm})$ and sealed with UV sensitive epoxy glue onto microscope slides.

Confocal Laser Scanning Microscopy (CLSM) measurements were performed on a Nikon TE2000U inverted microscope fitted with a C1 confocal scan head and a 100x Nikon oil objective. For illumination, a Spectra Physics 163C air cooled ion laser was used at a wavelength of $488 \mathrm{~nm}$. Samples were made in sedimentation cells consisting of a round glass capillary ( $2 \times 100 \mathrm{~mm}$ internal dimension) glued with araldite glue to a glass cover slip of $0.17 \mathrm{~mm}$ thick (Menzel-Gläser). Cube dispersions were placed in the cells and the sedimentation was imaged over time in the layer on top of the glass wall. Regions of $50 \times 50 \mu \mathrm{m}$ were imaged with $1024 \times 1024$ pixel resolution with time steps of 30s. For measurements longer than 8 hours height scans were taken every $30 \mathrm{~min}$ because the distance between the sample and objective was found to change over time, most likely due to small temperature fluctuations.

Dynamic light scatteing (DLS) and electrophoretic mobility measurements were performed with a Malvern Zetasizer Nanoseries equipped with a He-Ne laser operating at a wavelength $\lambda=633 \mathrm{~nm}$ operating in backscattering geometry at $173^{\circ}$. Freshly prepared samples of $50 \mu \mathrm{l}$ 5-7 wt\% cube dispersion mixed with $5 \mathrm{~mL}$ solvent and homogenized well. Directly prior to a measurement the dispersions were sonicated for $10 \mathrm{~s}$. 


\subsection{Colloidal cube properties}

\subsection{Size}

Several batches of colloidal hematite cubes were prepared according to the synthesis described in Section 5.2. Figure 5.1 shows typical TEM images of hematite cubes, silica coated hematite cube and hollow silica cubes resulting from the synthesis. Figure 5.1.a. shows an overview of hematite cubes which can be seen to have uniform size and shape. The silica shell can be distinguished as the light grey layer around the dark core in Figure 5.1.b. (Fig.5.1.b) and as a dark shell when the hematite core is removed (fig. 5.1.c.) From the TEM images, the average edge length $\langle D>$ can be determined by measuring the side to side length of the center of the cube. The TEM images also show that the cubes are not sharp-edged cubes but possess rounded corners

Cube sizes can also be determined with dynamic light scattering (DLS) which provides the hydrodynamic radius $R_{h}$ the particles in suspension based on the diffusion constant $D_{t}$ using the Stokes-Einstein equation. However, due to their large size and high density the cubes have an additional fast downward drift due to gravity that will increase the measured diffusion constant $D_{t}$ and hence decrease the measured particle size. Furthermore, hematite cubes will form clusters due to their magnetic interactions, which will be discussed in more detail in Section 5.3.3, which enhances the settling rates. See appendix A.5 for more details on the clustering effect on the DLS measurements.

\subsubsection{Shape}

An important feature of the obtained colloidal cubes is that their rounded shape can be approximated with that of a superball, which contour is represented by ${ }^{[3,19]}$ :

$$
\left|\frac{x}{a}\right|^{m}+\left|\frac{y}{a}\right|^{m}+\left|\frac{z}{a}\right|^{m} \leq 1
$$

where $m$ is the shape parameter, which indicates the extent of deformation from a sphere $(m=2)$ to a cube $(m \rightarrow \infty)$ and $a$ is half the edge length $D$.
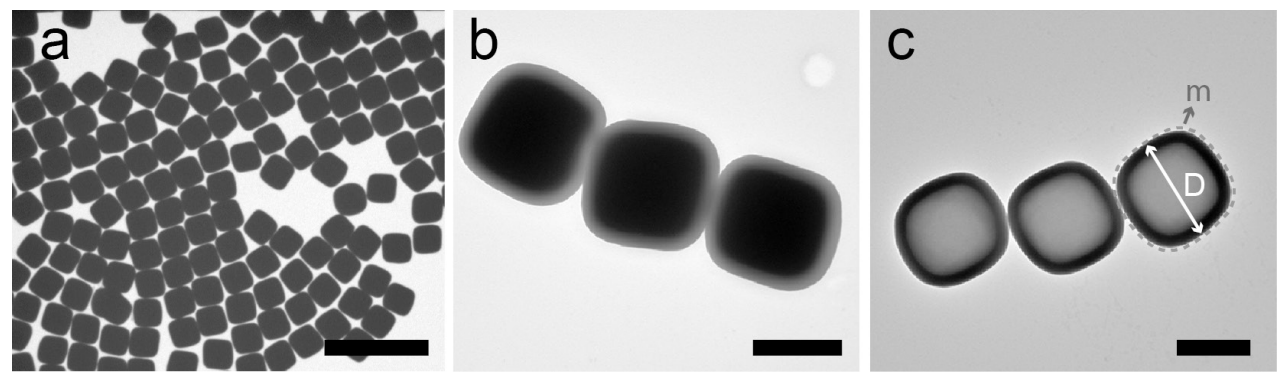

Figure 5.I. Typical transmission electron microscope images of the cube synthesis, shown here for a) hematite cubes, HC4 b) silica coated hematite cubes, HC4_SiF c) hollow silica cubes, HC4_SiF_H, including schematic illustration of the cube edge length $D$ and the contour from which the shape parameter $\mathrm{m}$ is determined. Scale bars are a) $5 \mu \mathrm{m} \mathrm{b}$, c) I $\mu \mathrm{m}$. 
For the $m$ determination two different approaches can be taken. The first approach is a simple calculation from the ratio of $a$ and the face diagonal $L$ given by ${ }^{[33]}$ :

$$
L=\sqrt{8} a\left(\frac{1}{2}\right)^{1 / m}
$$

The second approach involves a superdisk (2D equivalent of a superball) contour fit of the $2 \mathrm{D}$ projection of the cubes in the TEM images ${ }^{[3]}$. For this method TEM images we used of individual cubes that were clearly lying flat on the sample grid. The values extracted with both methods for $m$ were found to be in good agreement. The contour fit, however, is more sensitive to the deviation from a flat orientation of a cube and was therefore used to determine most $m$-values.

In Table 5.1 $D$ and the value of their shape parameter $m$ of most cubes synthesized for this thesis work are given, based on the TEM analysis from at least 100 individual cubes. It is noted that the codes in the cube name indicate if the cubes are silica coated (_Si) and hollow (_H). It is clear that cubes with different edge lengths can be synthesized and that $m$ increases, or in other words the cubic shape becomes more pronounced for larger cubes. The silica coating on the other hand effectively rounds off the cubes edges and increasing the silica thickness decreases $m$ even further, as seen for F, F_Si1 and F_Si2 for which $m$ is 3.7, 3.1 and 2.9, respectively.

Table 5.I. Particle properties of selected hematite cubes, silica coated hematite cubes and hollow silica cubes.

\begin{tabular}{|c|c|c|c|c|c|}
\hline Name & $\begin{array}{l}<D> \\
(n m)\end{array}$ & $\begin{array}{c}\sigma_{D} \\
(\%)\end{array}$ & $\begin{array}{c}<t_{\mathrm{SiO}_{2}}> \\
(\mathrm{nm})\end{array}$ & $m$ & $\begin{array}{l}L_{g-\mathrm{H} 2 \mathrm{O}} \\
(\mathrm{nm})\end{array}$ \\
\hline \multicolumn{6}{|l|}{ Hematite } \\
\hline L2 & 533 & 4 & - & 3.55 & 641 \\
\hline $\mathbf{F}$ & 558 & 6 & - & 3.7 & 559 \\
\hline HC1 & 584 & 10 & - & a & 487 \\
\hline HC2 & 920 & 7 & - & a & 125 \\
\hline HC5 & 932 & 6 & - & a & 120 \\
\hline HC4 & 1180 & 4 & - & & 59 \\
\hline \multicolumn{6}{|c|}{ Hematite + Silica } \\
\hline L2_Si & 652 & 7 & 59 & 3.1 & 351 \\
\hline F_Si1 & 668 & 4 & 55 & 3.13 & 326 \\
\hline F_Si2 & 774 & 5 & 108 & 2.9 & 209 \\
\hline HC5_Si & 1028 & 4 & 48 & 3.55 & 89 \\
\hline HC4_Si & 1266 & 4 & 43 & 3.55 & 48 \\
\hline HC4_SiF & 1413 & 4 & 116 & 3.33 & 34 \\
\hline \multicolumn{6}{|l|}{ Hollow Silica } \\
\hline F_Si2_H & 774 & 5 & 108 & 2.9 & 4086 \\
\hline HC5_Si_H & 1028 & 4 & 48 & 3.55 & 4736 \\
\hline HC4_Si_H & 1266 & 4 & 43 & 3.55 & 3441 \\
\hline HC4_SiF_H & 1413 & 4 & 116 & 2.65 & 560 \\
\hline
\end{tabular}


Surprisingly, the addition of a fluorescent dye to the silica shell was found to decrease the $m$-value determined from the TEM images when the hematite core was removed. This can be seen for HC4_SiF and HC4_SiF_H in Table 5.1, for which $m$ is found to be 3.33 and 2.65, respectively. The rounding effect was not observed for pure silica shells and it remains unclear whether the cubes are also rounded in suspension.

\subsubsection{Sedimentation}

Hematite has a relatively high mass density of $\rho=5.25 \mathrm{~g} / \mathrm{cm}^{3}$ while the porous silica coating of the cubes has a mass density of about $\rho=1.6 \mathrm{~g} / \mathrm{cm}^{3[34]}$. For all three types of cubes their size in combination with their mass density will cause the cubes to sediment under gravity. The mass density difference leads to different gravitational lengths, $L_{g}$, as shown of the various cubes in Table 5.1. For the hematite cubes $L_{g}$ is comparable or (much) smaller than $D$. This means that dense sediments of the cubes will form in which gravitational compression will occur due to the mass of the accumulated cubes above. For the hollow silica cubes the $L_{g}$ are one order of magnitude larger but still on the order of $D$.

\subsubsection{Interactions}

Particle interactions are determined by the surface as well as bulk properties of the cubes. Hematite has an iso-electric point (IP) around $\mathrm{pH} 7.5-8.5^{[35]}$ and possesses a magnetic dipole moment due to canted antiferromagnetism ${ }^{[3,36,37]}$. Silica has a point of zero charge around $\mathrm{pH}$ $1.7-3.5^{[32]}$ and is non-magnetic.

\section{Surface charge and zeta potential}

The fact that both hematite and silica have an IP implies that the surface charge sign and charge density of the cubes can be changed by the increasing or decreasing the $\mathrm{pH}$ above or below the IP. For instance hematite cubes dispersed at pH 3 are positively charged, while at pH 9 the cubes have a net negative charge. The surface charge density will also increase when moving further away from the IP of the cube material. In addition, the surface can be modified by the adsorption of surfactants or ions. Furthermore, the cubes are stabilized by double layer repulsion acting over a distance set by the Debye screening length, $\kappa^{-1}$, given by:

$$
\kappa^{-1}=\sqrt{\left(\varepsilon_{0} \varepsilon_{r} k T / 2 N_{A} e^{2} I\right)}
$$

here $\varepsilon_{0}$ is the vacuum permittivity, $\varepsilon_{r}$ the dielectric constant of the solvent, $k_{B}$ is the Boltzmann constant, $T$ the absolute temperature, $N_{A}$ is Avogadro's number, $e$ the elementary charge and $I$ is defined as the ionic strength of the electrolyte.

Electrophoretic measurements can be done to determine the electrophoretic mobility of the cubes and provide insight into the effect of the cube material and solutions on the surface charge sign. Measurements were performed of several types of cubes in different solvents; ethanol, deionized water and $6 \mathrm{mM}$ TMAH solution $(\mathrm{pH}=9)$. It has to be noted that the 
Table 5.2. Zeta potential of hematite cubes and hematite cubes coated with silica and hollow silica cubes in different solvents.

\begin{tabular}{l|ccc}
\hline Cubes & $\begin{array}{c}\text { Ethanol } \\
(\boldsymbol{m} \boldsymbol{V})\end{array}$ & $\begin{array}{c}\text { Demi Water } \\
(\boldsymbol{m} \boldsymbol{V})\end{array}$ & $\begin{array}{c}\text { TMAH } \\
(\boldsymbol{m} \boldsymbol{)})\end{array}$ \\
\hline L2 & - & 38 & -75 \\
HC1 & 37 & 39 & -59 \\
HC2 & 47 & 48 & -61 \\
L2_Si & -44 & -49 & -39 \\
F_Si1 & -54 & -58 & -26 \\
F_Si2 & -45 & -59 & -23 \\
\hline
\end{tabular}

obtained zeta-potential is obtained from the electrophoretic mobility via the Smoluchowski model, which is valid here because the cubes are very much larger than $\kappa^{-1}$.

Table 5.2 shows the zeta-potentials, $\zeta$, for three differently sized hematite cubes, L2, HC1 and HC2 and three different silica coated cubes L2_Si, F1_Si and F2_Si. For these hematite cubes the zeta potentials in the three solvents are of the same order of magnitude. In water and ethanol the cubes are positively charged while by addition of TMAH the $\mathrm{pH}$ is raised above the IP of hematite resulting in charge reverse as expected. However, it is known that TMAH will adsorb onto the hematite surface and will also contribute to the zeta potential change ${ }^{[38]}$. For the silica coated cubes the zeta potential was always found to be negative, as expected far above the IP of silica. In the case of TMAH a slightly less negative zeta potential is obtained.

\section{Magnetic interactions}

Hematite possesses a permanent magnetic moment that induces dipole-dipole attractions between the hematite cubes. Detailed high resolution TEM investigations of the cubes have revealed that subcrystals of hematite extend radially from the particle center in all directions ${ }^{[3,39]}$. The origin of this polycrystalline internal structure is still unclear but the acidic environment
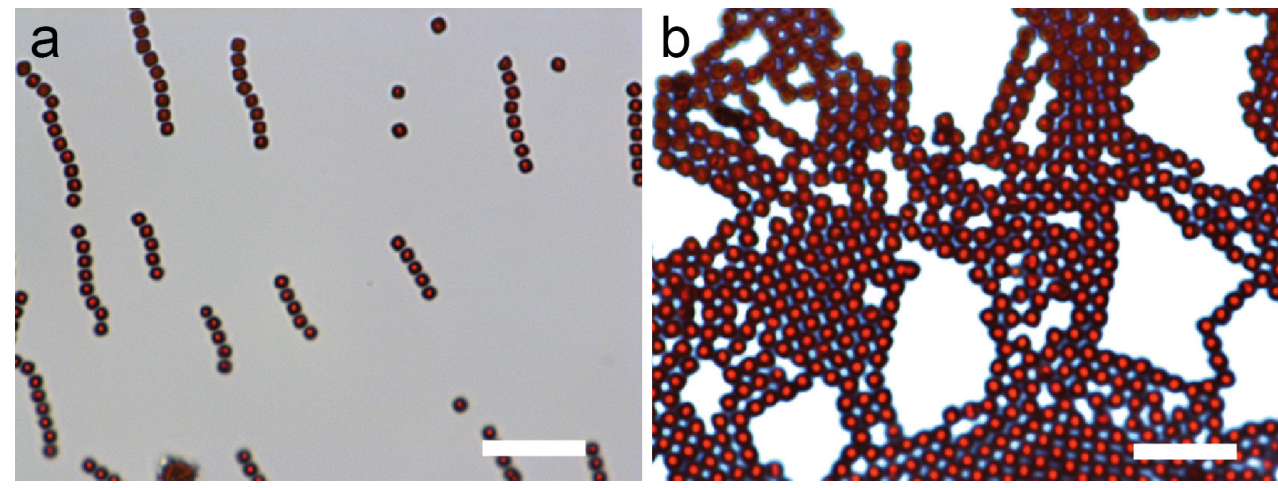

Figure 5.2. Optical microscopy images of colloidal silica coated hematite cubes dispersed in ethanol at a) low concentrations and b) high concentration. Scale bars are $10 \mu \mathrm{m}$. 
during the synthesis is thought to contribute to the subcrystal formation ${ }^{[39]}$. The TEM studies also revealed that the c-axis of the hematite corundum crystal structure is oriented along the long diagonal of the cubes. Other work on peanut-shaped hematite particles, which can be obtained by addition of $\mathrm{SO}_{4}^{2-}$ ions to the gel-sol reaction mixture, has shown that the magnetic dipole must be oriented perpendicular to this $\mathrm{c}$-axis ${ }^{[30]}$. However, the precise magnitude and orientation of the magnetic moment of the cubes are still uncertain and subject of on-going study ${ }^{[3,40]}$.

In stable hematite cube dispersions the dipole-dipole attraction causes the formation of flexible strings at low concentrations ${ }^{[3,36,40,41]}$. Figure 5.2.a shows microscopy images of these strings in dispersions of silica coated hematite cubes HC4_SiF in ethanol. The strings are aligned in a weak stray magnetic field caused by components of the microscope. Note that the red color of the cubes is due to the hematite core. In the strings rotations of the cubes could be observed over time. Figure 5.2.b. shows an assembly of cubes HC4_Si at high concentration, where the strings of cubes have clustered into ordered domains.

\section{Overall interaction potential}

To obtain a stable self-assembling system of hematite cubes the Van der Waals (and magnetic attraction, in case of hematite) should be sufficiently counteracted by the double layer repulsion. Ideally, one would like to calculate the total interaction potential based on the information provided above. However, quantitative information on the total pair interaction energy for the colloidal cubes studied in this part of the thesis is at this stage scarce. In the first place, accurate values of surface potentials or surface charge densities are difficult to obtain. In addition, conversion of zeta potentials into surface charge densities is anything but straight forward. For further discussion on this point we refer to ref.[42], which is an experimental study on the double layer interaction between charged silica spheres in ethanol. For charged cubes the pair interaction between two particles is further complicated by orientational dependence of both the double layer repulsion and the Van der Waals attraction. For example, the repulsion between two cubes in face-to-face orientation will be much larger than when the cubes approach each other facing their rounded edges. No theory or simulation results are available yet for the orientation-dependent DLVO interaction between two superballs. The interpretation of the cube interactions in the following chapters is therefore restricted to a qualitative consideration based on the magnitude of the Debye length and expected changes is surface charge densities in each specific sample.

\subsubsection{Sedimentation experiments}

How the presence of attractive or repulsive particles interactions influences the assembly of silica coated hematite cubes and hollow silica cubes during sedimentation was investigated via sedimentation studies of diluted suspensions of cubes HC4_SiF and HC4_SiF_H. These cubes have fluorescein incorporated into their silica shell and can therefore be visualized with a confocal laser scanning microscope (CLSM). It was found that the fluorescent labelling also 

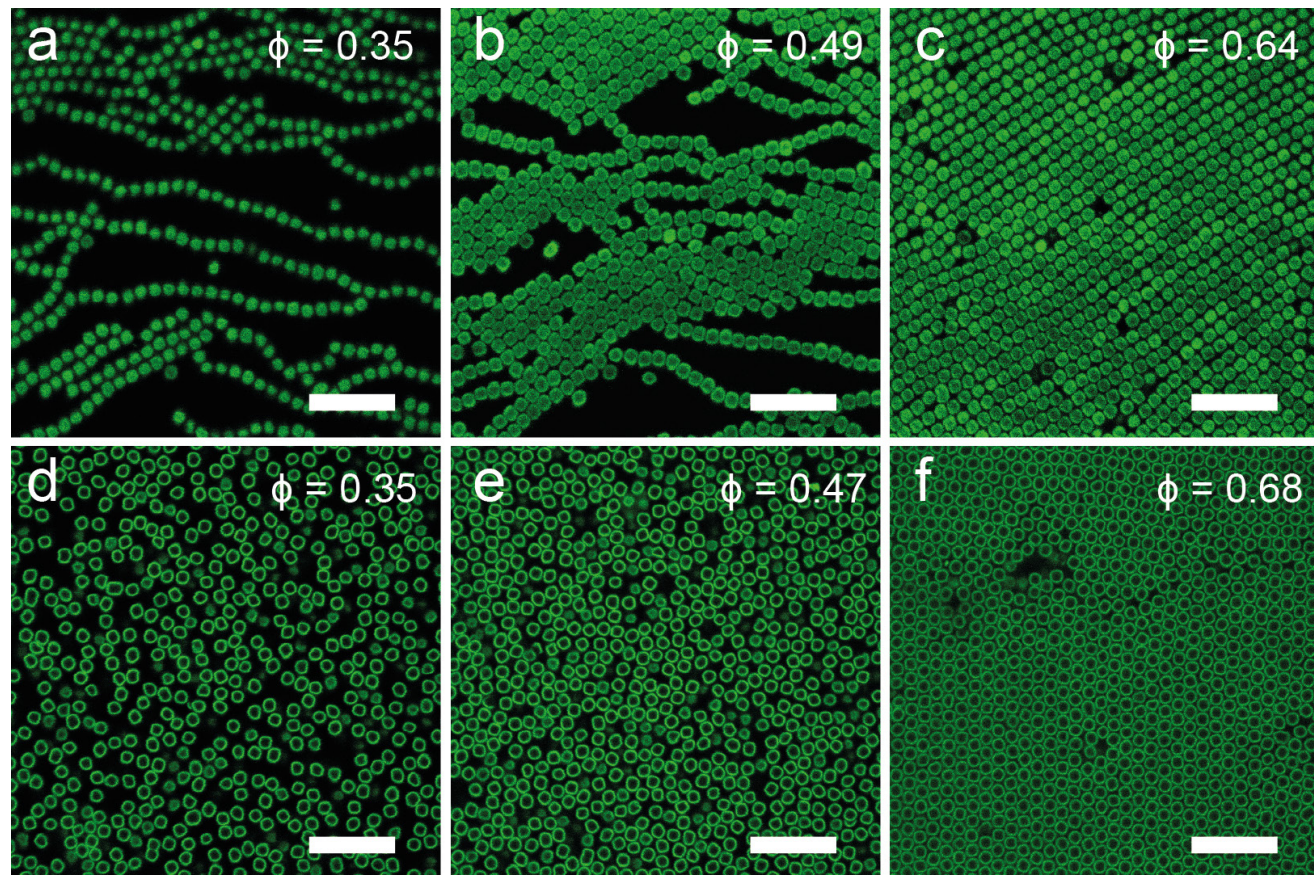

Figure 5.3. Confocal images of sediments of a-c) fluorescently silica coated hematite cubes and d-f) hollow fluorescent silica cubes, imaged over time while the volume fraction $\phi$ increased due to sedimentation. Scale bars are $10 \mu \mathrm{m}$.

affected the particle interactions as these cubes became slightly sticky in water and therefore the experiments were performed in ethanol.

The sedimentation experiments were performed by filling a measuring cell with a diluted dispersion $(0.001 \mathrm{wt} \%)$ of cubes in ethanol. After sealing the cell, it was placed immediately on the confocal microscope and the sedimentation onto the glass wall was imaged over time. From the confocal images the particle positions were determined per frame using particle tracking algorithms in IDL or Mathematica. From the number of particles per frame, the area of one image $\left(50 \times 50 \mu \mathrm{m}^{2}\right)$ and the area of one colloid (estimated at $1.80 \mu \mathrm{m}^{2}$ based on $D$ and $\mathrm{m}$ ), the volume fraction, $\phi$, on the glass surface was determined.

Figure 5.3 shows the obtained confocal images of the sediments of hematite filled and hollow silica cubes at low, intermediate and high volume fraction. The hematite cubes form flexible strings due to the magnetic interactions at low concentration, $\phi=0.35$ (Fig. 5.3.a). The strings are again aligned to a stray magnetic field originating from the confocal microscopy components. As the volume fraction increases to $\phi=0.49$ (Fig. 5.3.b), the strings connect and align followed by the formation of an ordered structure at high volume fraction, $\phi=0.64$ (Fig. 5.3.c). In this structure the cubes do not rotate but instead fluctuate slightly on their lattice position. For the hollow silica cubes a different process is observed. The cubes now lack magnetic attractions and 

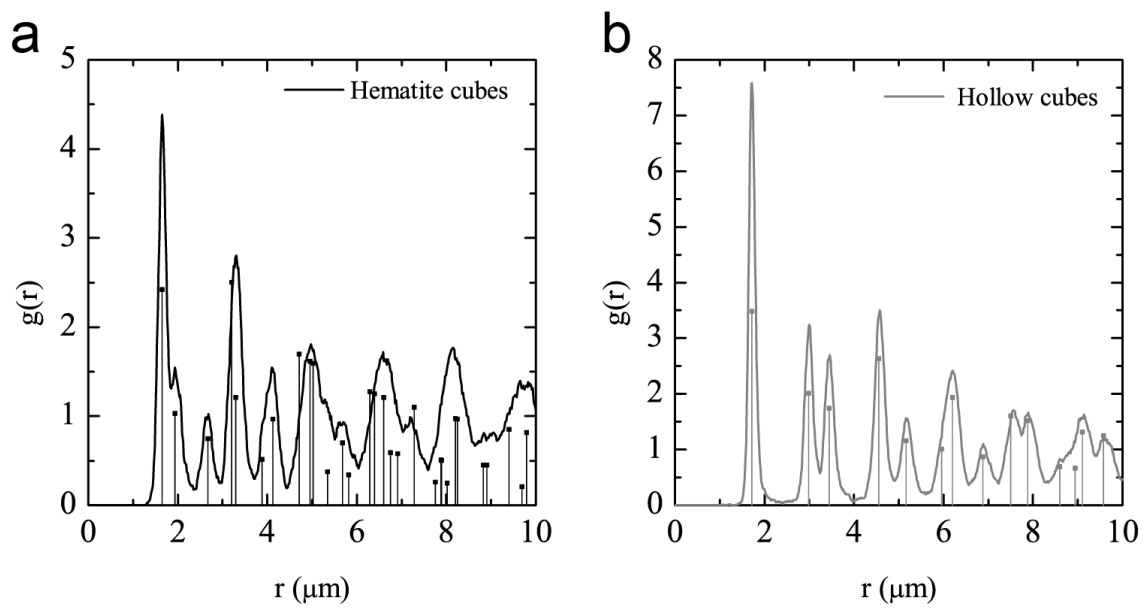

Figure. 5.4. Pair correlation functions of the sediments at high volume fraction of a) hematite cubes and b) hollow silica cubes. Together with the theoretical values of a rhombic crystal lattice in a) and a hexagonal lattice in b).

a fluid of single cubes that display Brownian motion and rotate freely was observed at $\phi=0.35$ (Fig. 5.3.d). An increase in volume fraction to $\phi=0.47$ causes the cubes to order locally (Fig. 5.3.e), until at $\phi=0.68$ an ordered structure is obtained (Fig. 5.3.f). In the ordered structure the cubes could be observed to rotate on their lattice position but translations were not observed.

From the particle positions the pair correlation function $g(r)$ was calculated and is shown for the two highest volume fractions of both cubes in Figure 5.4. The $g(r)$ curves both show distinct peaks. Interestingly, the peaks of the hematite cubes can be fitted to that of a rhombic lattice of cubes with $d_{\text {eff }}=1.63 \mu \mathrm{m}$ and $m=4$ (Fig.5.4.a), while the hollow cube peaks fit very well to that of a hexagonal lattice with $d_{\text {eff }}=1.72 \mu \mathrm{m}$ (Fig.5.4.b). The difference in effective particle size corresponds to a larger local particle volume and agrees with the observed rotations of the hollow cubes. Apparently, the hollow silica cubes have formed a rotator hexagonal phase. The absence of rotations of the hematite cubes on the rhombic lattice explains the closer distance and must be a consequence of the magnetic dipole-dipole interactions. The fact that the size of the cubes in the rhombic lattice is still larger than $D=1.413 \mu \mathrm{m}$ determined by TEM analysis, can be explained by the low ionic strength of the solvent. At low ionic strength the colloidal size can be assigned an effective interaction diameter $d_{\text {eff }}=D+\alpha \kappa^{-1}$ where $\alpha \sim 3-4^{[34]}$. Here, the Debye screening length is estimated to be $\kappa^{-1}=50 \mathrm{~nm}$ and the calculated effective diameter $d_{\text {eff }}=1.61 \mu \mathrm{m}$ for $\alpha=4$ is in good agreement with the experimentally measured $d_{\text {eff }}=1.63 \mu \mathrm{m}$. 


\subsection{Conclusions}

Three different types of colloidal cubes have been synthesized: hematite cubes, silica coated hematite cubes and hollow silica cubes with different sizes and different shape parameter. The edge length of the cubes can be varied between $D=500$ and $1500 \mathrm{~nm}$ while the shape can be tuned between $m=2.7$ and 3.6. With respect to the double-layer interaction between charged cubes, we are yet limited to a qualitative assessment of surface charge and zeta potential since no model or theory is available for the anisotropic double-layer repulsion between two cubes.

The sedimentation behavior of silica coated hematite cubes and hollow silica cubes is observed to be influenced by the different particle interactions. For the hematite cubes magnetic dipoledipole interactions are present that cause already at low volume fractions string formation and clustering, which at high volume fractions form a rhombic crystal structure. When the magnetic hematite core is removed, the hollow silica cubes show a transition from a fluid to a hexagonal rotator crystal.

\section{Acknowledgements}

Laura Rossi is thanked for help with the cube synthesis and many fruitful discussions. Sonja Castillo, Vera Meester, Fabian Hagemans and Lisette Pompe are thanked for their contributions to the cube syntheses. Maarten van den Berg, Kristian van Berlo, Robbert van Dinther en Joeri Opdam are thanked for their contributions to the sedimentation experiments. William Irvine is thanked for providing the MatLab scripts for analysis of the superball shape parameter $m$. 


\section{Appendix}

\section{A.5. Effects of cube settling on DLS measurements}

Here the challenging nature of DLS measurements due to sedimentation and attractions are demonstrated. Figure 5.2.a shows the correlation coefficient versus delay time of three subsequent DLS measurements of hematite cubes L2 in water with $D=533 \mathrm{~nm}$ and a polydispersity of $4 \%$ as determined with TEM analysis. The sample was homogenized and prior to the measurement sonicated for 10s to redisperse the colloids and to destroy any formed clusters. The intensity of the correlation coefficient is rather low, which can be attributed to sedimentation and absorbance (another disadvantage of hematite in optical measurements). A typical decay time is present from single particles diffusing through the measured sample volume. However, a long tail at long decay times is present which indicates the presence of large clusters. The fact that this is already observed after $3 \mathrm{~min}$ and that the tail increases with increasing time shows that clusters increase in size over time. To extract $R_{h}$ from these graphs is not trivial. However, the Malvern zetasizer software can extract a diffusion constant from the graphs and determines the $R_{h}$. Figure 5.2.b. shows the increase in size over time of $R_{h}=541.1 \mathrm{~nm}, 548.8 \mathrm{~nm}$ and $587.8 \mathrm{~nm}$ with polydispersities of $14.5 \%, 17.1 \%$ and $5 \%$. These values are close to the values determined with TEM but are clearly affected by the sample history and handling. Therefore, the cube size determination was restricted to TEM image analysis.
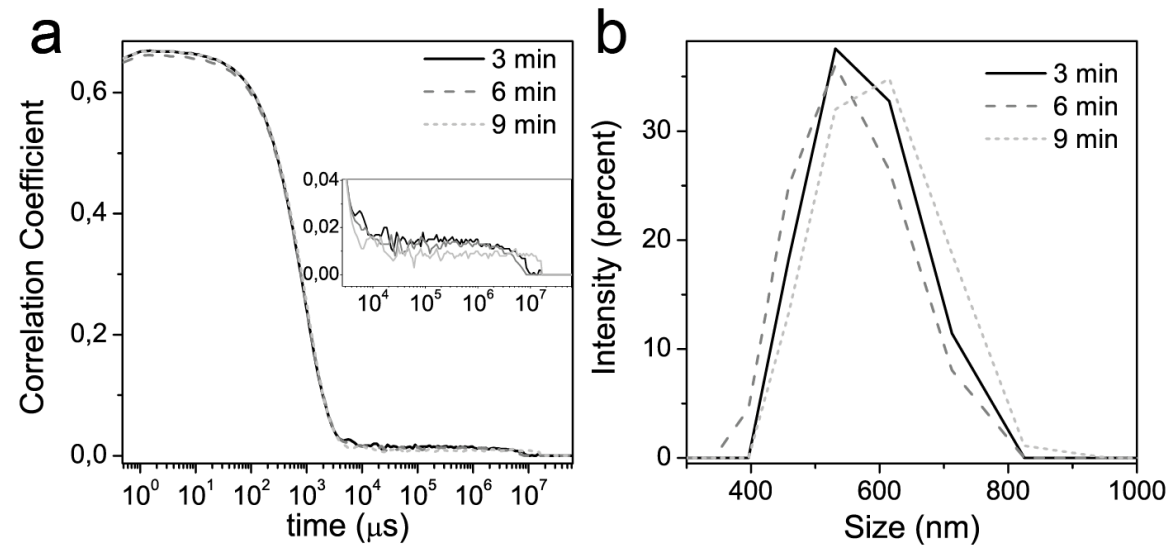

Figure 5.5. a) Three raw DLS correlograms of hematite cubes $\langle D\rangle=533 \mathrm{~nm}$ at different times after sonication, inset shows a magnification of the long time features and b) size distribution as calculated by zetasizer software. 


\section{References}

1. D. J. Kraft, R. Ni, F. Smallenburg, M. Hermes, K. Yoon, D. A. Weitz, A. van Blaaderen, J. Groenewold, M. Dijkstra and W. K. Kegel, Proc. Natl. Acad. Sci. U. S. A., 2012, 109, 10787-10792.

2. A. F. Demirors, P. M. Johnson, C. M. van Kats, A. van Blaaderen and A. Imhof, Langmuir, 2010, 26, 14466-14471.

3. L. Rossi, Colloidal Superballs, Ph.D. Thesis, Utrecht University, 2012.

4. T. Sugimoto and K. Sakata, J. Colloid Interface Sci., 1992, 152, 587-590.

5. A. Ahniyaz, Y. Sakamoto and L. Bergstrom, Proc. Natl. Acad. Sci. U. S. A., 2007, 104, 17570-17574.

6. Y. Sun and Y. Xia, Science, 2002, 298, 2176-2179.

7. B. Wiley, Y. Sun, B. Mayers and Y. Xia, Chem. Eur. J, 2005, 11, 454-463.

8. Y. Zhang, F. Lu, D. van der Lelie and O. Gang, Phys. Rev. Lett., 2011, 107, 135701.

9. L. Rossi, S. Sacanna, W. T. M. Irvine, P. M. Chaikin, D. J. Pine and A. P. Philipse, Soft Matter, 2011, 7, 4139-4142.

10. J. Henzie, M. Grünwald, A. Widmer-Cooper, P. L. Geissler and P. Yang, Nature Mater., 2012, 11, 131-137.

11. H. Yang, S. He, H. Chen and H. Tuan, Chem. Mater., 2014, 26, 1785-1793.

12. K. Miszta, J. de Graaf, G. Bertoni, D. Dorfs, R. Brescia, S. Marras, L. Ceseracciu, R. Cingolani, R. van Roij, M. Dijkstra and L. Manna, Nature Mater., 2011, 10, 872-876.

13. S. Sacanna, D. J. Pine and G. Yi, Soft Matter, 2013, 9, 8096-8106.

14. S. C. Glotzer and M. J. Solomon, Nature Mater., 2007, 6, 557-562.

15. U. Agarwal and F. A. Escobedo, Nature Mater., 2011, 10, 230-235.

16. P. F. Damasceno, M. Engel and S. C. Glotzer, Science, 2012, 337, 453-457.

17. E. A. Jagla, Phys. Rev. E., 1998, 58, 4701-4705.

18. B. Groh and B. Mulder, J. Chem. Phys., 2001, 114, 3653-3658.

19. Y. Jiao, F. H. Stillinger and S. Torquato, Phys. Rev. E, 2009, 79, 041309.

20. R. D. Batten, F. H. Stillinger and S. Torquato, Phys. Rev. E, 2010, 81, 061105.

21. R. Ni, A. P. Gantapara, J. de Graaf, R. van Roij and M. Dijkstra, Soft Matter, 2012, 8, 12135-12135.

22. M. Marechal, U. Zimmermann and H. Loewen, J. Chem. Phys., 2012, 136, 144506.

23. T. Sugimoto, M. M. Khan, A. Muramatsu and H. Itoh, Colloids Surf. A., 1993, 79, 233-247.

24. S. Disch, E. Wetterskog, R. P. Hermann, G. Salazar-Alvarez, P. Busch, T. Brueckel, L. Bergstroem and S. Kamali, Nano Lett., 2011, 11, 1651-1656.

25. Y. Xia, Y. Xiong, B. Lim and S. E. Skrabalak, Angew. Chem. Int. Ed., 2009, 48, 60-103.

26. I. Sevonkaev, D. V. Goia and E. Matijević, J. Colloid Interface Sci., 2008, 317, 130-136.

27. T. Sugimoto and Y. Wang, J. Colloid Interface Sci., 1998, 207, 137-149.

28. T. Sugimoto, Y. Wang, H. Itoh and A. Muramatsu, Colloids Surf. A., 1998, 134, 265-279.

29. T. Sugimoto, M. M. Khan and A. Muramatsu, Colloids Surf. A., 1993, 70, 167-169.

30. S. H. Lee and C. M. Liddell, Small, 2009, 5, 1957-1962.

31. C. Graf, D. L. J. Vossen, A. Imhof and A. van Blaaderen, Langmuir, 2003, 19, 6693-6700.

32. G. B. Alexander, W. M. Heston and R. K. Iler, J. Phys. Chem, 1954, 6, 453-455.

33. A. G. M. Brinkman, Preparations and properties of colloidal cubes, Internal report, University Utrecht, 2011.

34. A. P. Philipse and G. H. Koenderink, Adv. Colloid Interface Sci., 2003, 100, 613-639.

35. L. Cromieres, V. Moulin, B. Fourest and E. Giffaut, Colloids Surf. A., 2002, 202, 101-115.

36. M. Ozaki, H. Suzuki, K. Takahashi and E. Matijevic, J. Colloid Interface Sci., 1986, 113, 76-80.

37. N. K. Chaudhari, H. C. Kim, C. S. Kim, J. Park and J. Yu, CrystEngComm, 2012, 14, 2024-2031.

38. S. Sacanna, L. Rossi and A. P. Philipse, Langmuir, 2007, 23, 9974-9982.

39. G. S. Park, D. Shindo, Y. Waseda and T. Sugimoto, J. Colloid Interface Sci., 1996, 177, 198-207.

40. A. I. Abrikosov, S. Sacanna, A. P. Philipse and P. Linse, Soft Matter, 2013, 9, 8904-8913.

41. M. Aoshima, M. Ozaki and A. Satoh, J. Phys. Chem. C, 2012, 116, 17862-17871.

42. A. P. Philipse and A. Vrij, J. Chem. Phys., 1988, 88, 6459-6470. 


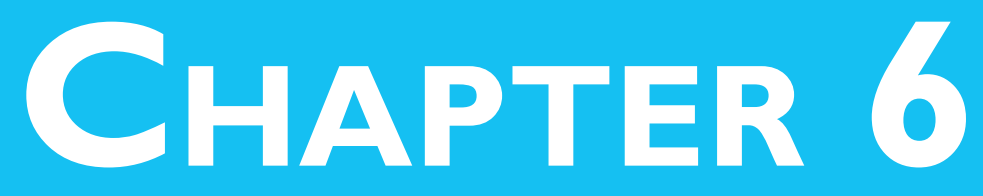

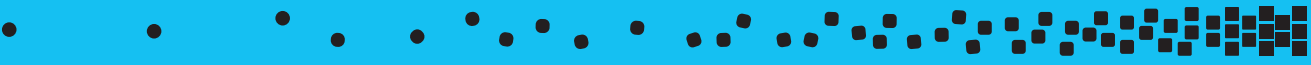 Self-ASSEMBly of COlloidal Cubes VIA VERTICAL DEPOSITION}

\section{Abstract}

The self-assembly of hollow silica cubes driven by convective assembly is investigated. Controlled deposition of the cubes via the vertical deposition technique results in large crystalline films. The local arrangements formed by the cubes are characterized in detail with scanning electron microscopy and small angle X-ray scattering. It is found that the local arrangements of the cubes in the structures vary from hexagonal to square. 


\section{I. Introduction}

The vertical deposition (VD) technique is well known for the convenient preparation of single crystal of colloids and with controllable thickness ${ }^{[1-4]}$, as described in Chapter 1, Section 1.4. The VD technique is based on the self-assembly of colloidal particles on a substrate, driven by capillary forces and convective flow, induced by fast solvent evaporation at the meniscus ${ }^{[1,5]}$. Improvements of the deposition method have allowed the fabrication of crystals of large $(\sim 1 \mu \mathrm{m})$ colloids, which typically sediment before deposition can occur, by preventing the sedimentation with a temperature gradient or by increasing the meniscus dropping velocity ${ }^{[6,7]}$.

The VD method has been applied to colloidal spheres for the fabrication of novel functional nanomaterials, such as photonic materials, with a bottom-up fabrication approach ${ }^{[2]}$. Because particle shape is known to greatly influence the crystal symmetry of close-packed structures, it is of fundamental and practical interest to study close-packed structures of non-spherical particles. The VD technique is very suitable for this purpose and has already been applied to several non-spherical colloids, such as rods and ellipsoids, for a review see Dugyala et al ${ }^{[8]}$.

Recently, a new anisotropic colloidal system of monodisperse hollow silica cubes was developed ${ }^{[9]}$. In addition, several studies were performed of the optimal packings these cubes can form ${ }^{[10,11]}$. Here we investigate the self-assembly of these hollow silica cubes into crystalline structures via the VD technique with various setups and the effect of their anisotropic shape on the formed structures. The obtained crystalline structures are characterized by scanning electron microscopy and microradian X-ray diffraction measurements. The different arrangements of cubes in single layers and multi-layered structures are analysed in detail. Moreover, the specific type of stacking and the long-range order of the microstructures are characterized.

\subsection{Experimental}

\subsubsection{Colloidal hollow silica cubes}

Precursor hematite $\left(\alpha-\mathrm{Fe}_{2} \mathrm{O}_{3}\right)$ particles were prepared from a condensed ferric hydroxide ge $^{[12,13]}$ and coated with silica according to an adaptation of the Stöber method ${ }^{[14,15]}$. Hollow silica cubes were obtained by dissolving the hematite cores in a hydrochloric acid solution ${ }^{[9]}$. The obtained white cube dispersion was transferred to ethanol for storage. For more details see Chapter 5, Section 5.2. The average cube face side, $\langle D\rangle$, its standard deviation, $\sigma$, and shape parameter $m$ (see Chapter 5, Section 5.3.1) were determined from transmission electron microscopy images (Fig. 6.1.a) and are given in Table 6.1.

\subsubsection{Sample preparation}

The cubes consist of thin hollow silica shells, which makes determination of the volume fraction from the weight concentration of the stock suspensions difficult. Therefore, the stock suspensions were centrifuged at $680 \mathrm{~g}$ in thin $4 \times 0.2 \mathrm{~mm}$ capillaries. Assuming that the sediment has a random packing density of $72 \mathrm{v} \%$ based on their shape ${ }^{[16]}$, the volume fraction was estimated from the obtained sediment volume. The desired volume fractions were achieved by diluting the stock dispersions with appropriate solvent. For water samples, a stock suspension 

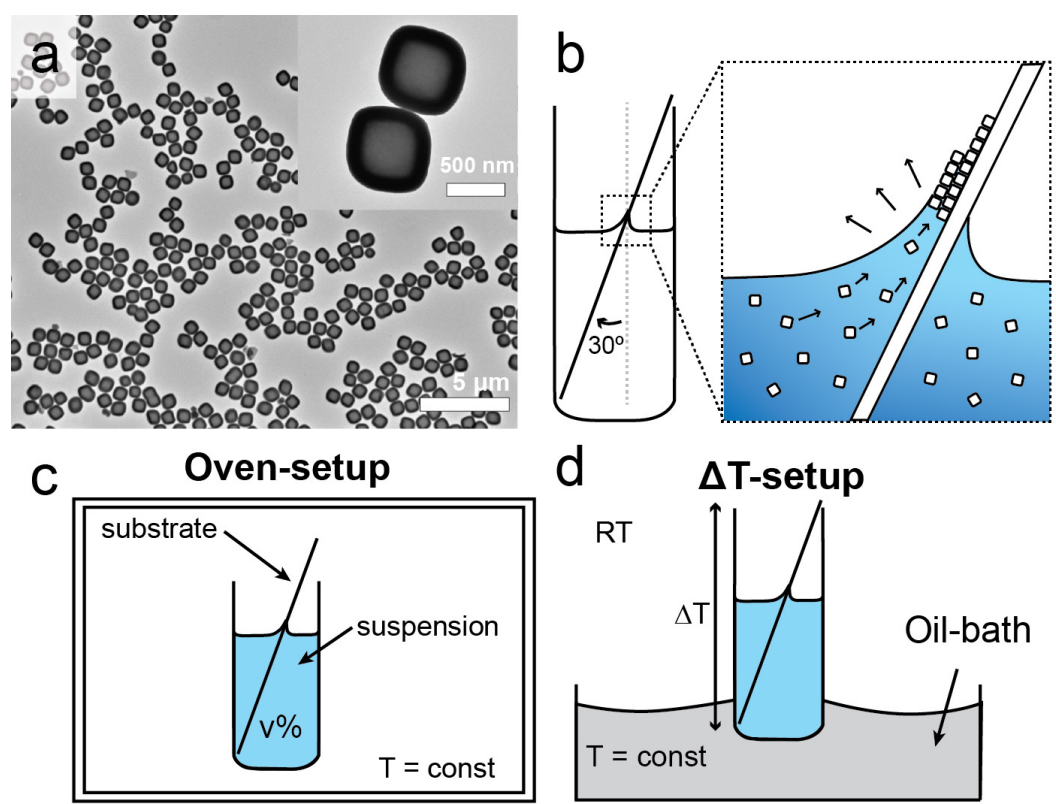

Figure 6.I. a) TEM image of the hollow silica cubes (insert) higher magnification of two cubes. b) Schematic illustration of the vertical deposition process. Cubes are transported to the meniscus by solvent evaporation and form a crystal. c-d) Schematic representation of the two different growth setups. The cube dispersion with a substrate is placed in a small vial is placed either c) in a heated oven at constant temperature, or d) in an oil-bath creating a temperature gradient along the dispersion vial.

Table 6.I. Characteristics of the precursor hematite cubes and hollow silica cubes

\begin{tabular}{l|lccc}
\hline Sample & Composition & $\begin{array}{c}\langle\boldsymbol{D}> \\
(\boldsymbol{n m})\end{array}$ & $\begin{array}{c}\boldsymbol{\sigma}_{\boldsymbol{D}} \\
(\boldsymbol{n m})\end{array}$ & $\boldsymbol{m}$ \\
\hline F & Hematite & 558 & 36 & 3.7 \\
F_Si2_H & Hollow Silica & 774 & 35 & 2.9 \\
\hline
\end{tabular}

in water was made prior to the experiments to avoid etching of the cubes.

\subsubsection{Vertical deposition conditions}

Small $2.5 \mathrm{ml}$ vials and thin glass microscope slides $(10 \times 35 \times 0.15 \mathrm{~mm})$ were etched for 24 hours in a $7 \mathrm{wt} \% \mathrm{KOH}$ (Merck) aqueous solution in ethanol. Subsequently, the slides were rinsed thoroughly with water and dried in air. The vials were filled with $2.5 \mathrm{ml}$ of cube suspension and a clean substrate was placed into the colloidal sol with an angle of $30^{\circ}$, see Figure 6.1.c. Two different vertical deposition-setups were used, as shown in Figure 6.1.d-e. For the first setup, the vial was placed in an oven at $50-70{ }^{\circ} \mathrm{C}$ for at least $24 \mathrm{~h}$ to allow the solvent to evaporate. In the second setup, a temperature gradient of about $5{ }^{\circ} \mathrm{C}$ was produced across the height $(\approx 2.5$ $\mathrm{cm}$ ) of the vial by placing the bottom in an oil bath of $60^{\circ} \mathrm{C}$, while keeping the area around the 
vial at room temperature. The latter method prevented sedimentation of the particles but also induced slow solvent evaporation over $24 \mathrm{~h}$. These setups will be referred to as the oven-setup and $\Delta T$-setup, respectively.

\subsubsection{Characterization}

Scanning electron microscopy (SEM) was performed with a Phenom FEI microscope. High resolution SEM (HR-SEM) images were obtained using a FEI XL30S FEG. A thin layer of platinum (usually $6 \mathrm{~nm}$ thick) was sputtered onto the samples prior to imaging. To reveal the thickness, the samples were scratched with a razor blade. By tilting the sample $40^{\circ}$, it was possible to image the edge of the films.

Small angle X-ray scattering (SAXS) measurements were performed at the European Synchrotron Radiation Facility (ESRF) micro optics test bench at the ID06 beamline. An $\mathrm{X}$-ray energy of $12.2 \mathrm{keV}$ was selected by a silicon double crystal monochromator. To get high resolution diffraction patterns we used a microradian X-ray diffraction setup with compound refractive lenses (CRLs) ${ }^{[17-19]}$. The CRLs consisted of 8 beryllium parabolic lenses with radius of parabola apex of $300 \mu \mathrm{m}$ and one lens with radius of parabola apex of $1000 \mu \mathrm{m}^{[20]}$. The CRLs focus the X-rays at the phosphor screen of the CCD X-ray detector (Photonic Science, $9 \times 9 \mu \mathrm{m}^{2}$ pixel area and dimensions of $4008 \times 2671$ pixels) located at $9 \mathrm{~m}$ from the lens. The samples were placed behind the CRLs at $7.54 \mathrm{~m}$ before the detector. The beam size on the sample was around $400 \times 400 \mu \mathrm{m}$.

\subsection{Results}

\subsubsection{Visual sample appearance}

The vertical deposition experiments performed, with both the oven- and $\Delta T$-setup, resulted in films of deposited cubes. However, only films prepared with aqueous suspensions displayed Bragg reflections of visible light. The films prepared with the oven-setup from water had a length of only 2-5 mm, with most of the substrate uncovered. This indicates that the cubes sediment faster than the time needed for evaporation of the water. As a result, no cubes are left in the suspension to be transferred to the meniscus. Raising the oven temperature to $70{ }^{\circ} \mathrm{C}$, which increases the evaporation speed of water and therefore shortens the overall time needed for deposition, did not greatly improve the film length or quality. In contrast, the $\Delta T$-setup resulted in multi-layered, completely substrate covering films. Clearly, the use of a temperature gradient during deposition is an effective method to prevent sedimentation of the large colloidal particles and allows the formation of large uniform films.

Figure 6.2.a shows a film prepared from a $0.5 \mathrm{v} \%$ dispersion in water using the $\Delta T$-setup over a period of $24 \mathrm{~h}$. The film covers the width and length of the substrate, up to the point where the VD was stopped, at $13 \mathrm{~mm}$ from the top. It displays strong Bragg reflections under white light illumination, indicating crystalline ordering. The colour-change of the reflected light, from blue-green (bottom) to red (top), occurs because the angle of reflection changes with respect to the camera. The non-uniform intensity of the scattering indicates the presence of small regions 

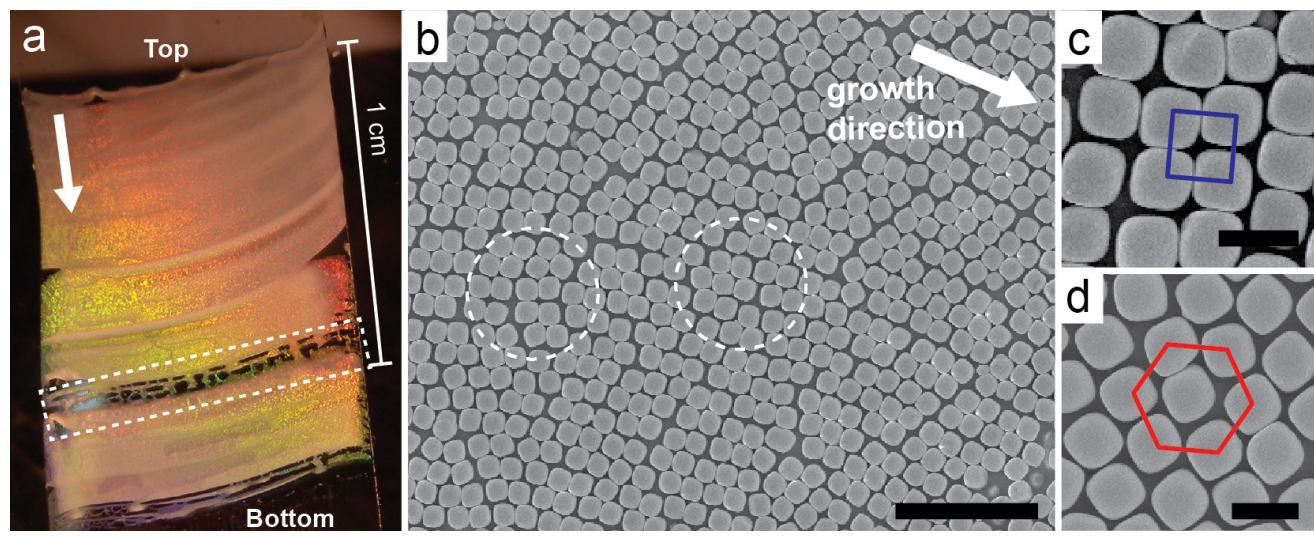

Figure 6.2. a) Photograph of the colloidal crystal film made with the $\Delta T$-setup with light illumination under an angle, showing Bragg reflections. The arrow indicates the growth direction. b) SEM image of a single layer of assembled cubes of a sample made with the oven-setup. Different arrangements of cubes occur, ranging from c) a square-like to d) hexagonal-like. Scale bars are b) $5 \mu \mathrm{m}$ and c,d) I $\mu \mathrm{m}$.

with different crystal orientation. A variation in film thickness along the growth direction (arrow Fig. 6.2.a) can be observed, and can be explained by fluctuations in the evaporation speed of the solvent, due to small uncontrolled variation of the humidity. Furthermore, a temporary absence of particles $4 \mathrm{~mm}$ above the bottom of the film can be seen (marked area). This can be explained by a change in the meniscus shape of the solvent, due to the closer proximity of the container wall toward the bottom of the substrate. The wetting of both the wall and the substrate will change the shape of the meniscus and causes inconsistent movement. This movement is a stick-slip motion, as has been previously reported for the films of spheres containing stripe-like patterns ${ }^{[5,21]}$. However, the humidity and air flux were not specifically controlled and are also known to cause meniscus fluctuations.

\subsubsection{D Structure characterization.}

The crystalline structure and thickness of deposited films were studied with SEM. Figure 6.2.b shows a typical SEM image of a single layer of assembled particles. The square shape of all cubes can be observed, indicating that the cubes are lying flat on the substrate. The orientation of the cubes is not fixed by the growth direction but is correlated with that of neighboring cubes. Different local arrangements of the cubes can be observed with four-fold or six-fold symmetry as indicated with the two circles. These structure seem to extend over typically 3-4 cube lengths. Figures 6.2.c and 6.2.d show larger magnifications of these arrangements, where the orientation are indicated with a square and hexagon, respectively. Evidently, the monodisperse cubes can be self-assembled with the vertical deposition technique into mono-layers of square to hexagonally coordinated arrays, where the cube orientation is predetermined by substrate and neighboring cubes. 

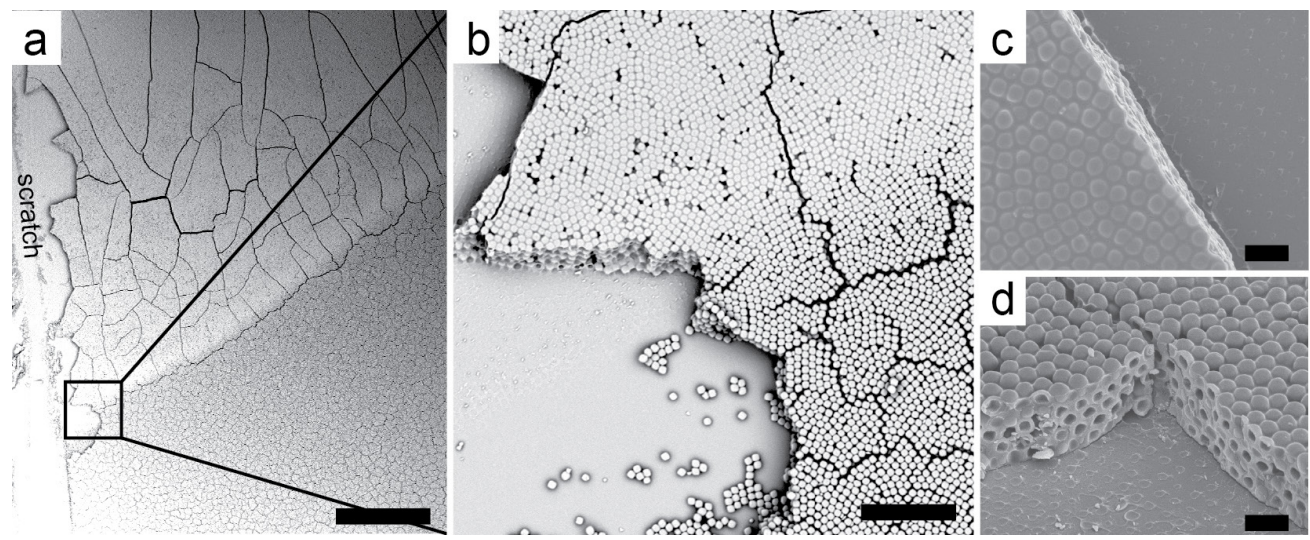

Figure 6.3. a,b) SEM images taken near the top of the film in Fig 6.2.a, showing two different regions, with large (top) and small (bottom) cracks, b) higher magnification image of the interface, showing connected cubes with matrix at the top and separated cubes at the bottom. c, d) HR-SEM images of a cracked region with more infiltration and a fracture cross section of infiltrated region, showing the hollow-inside of the particles. Scale bars are a) $100 \mu \mathrm{m}$ b) $10 \mu \mathrm{m}$ c,d) $2 \mu \mathrm{m}$.

\subsubsection{D Structure characterization.}

The $\Delta T$-setup was found to produce large substrate covering films with multiple layers that showed very distinct Bragg reflections. Therefore, the sample prepared with the $\Delta T$-setup, shown in Figure 6.2.a, was studied in more detail with both SEM and SAXS. A complete SEM scan of the sample showed that the crystal contained two distinctly different regions. Figure 6.3.a shows a SEM image $2 \mathrm{~mm}$ from the top of the film. The top of the image shows a lighter region, with large cracks running through it, and a darker area that contains smaller cracks. The scratch on the left was made with a razor blade to reveal the inner structure. Higher magnification images (Fig. 6.3.b.) show that the different crack-size arises from the fact that a connecting matrix is present between the cubes, which consists of silica, as will be discussed in more detail in Section 6.4.3. The connecting matrix is present in the top part, while in the bottom part separate cubes can be observed. Figure 6.3.c and 6.3.d show SEM images taken at $\sim 1 \mathrm{~mm}$ from the top of the film, where the cubes are hardly visible due to large amount of matrix material. Due to this matrix, the cubes are strongly inter-connected and when fractured, the structures will break along the path of least resistance, which is no longer along the cube edge (Fig. 6.3.c). Consequently, the hollow- inside of the cubes can even be seen (Fig. 6.3.d) but also edges of cubes sticking out. The same connecting matrix between the cubes was observed at $3.5 \mathrm{~mm}$ from the bottom of the film, where deposition had stopped momentarily (Fig. 6.2.a). Samples prepared with the oven-setup also showed the presence of a connecting matrix at the start of the deposition, although only for short regions and mainly at the highest temperature, $70^{\circ} \mathrm{C}$. Apparently, the matrix only formed at the onset of deposition. The parts of the sample with a matrix will be further referred to as infiltrated regions.

Figure 6.4 shows SEM images taken at the top $(1 \mathrm{~mm})$ and center $(5 \mathrm{~mm})$ of the crystalline 


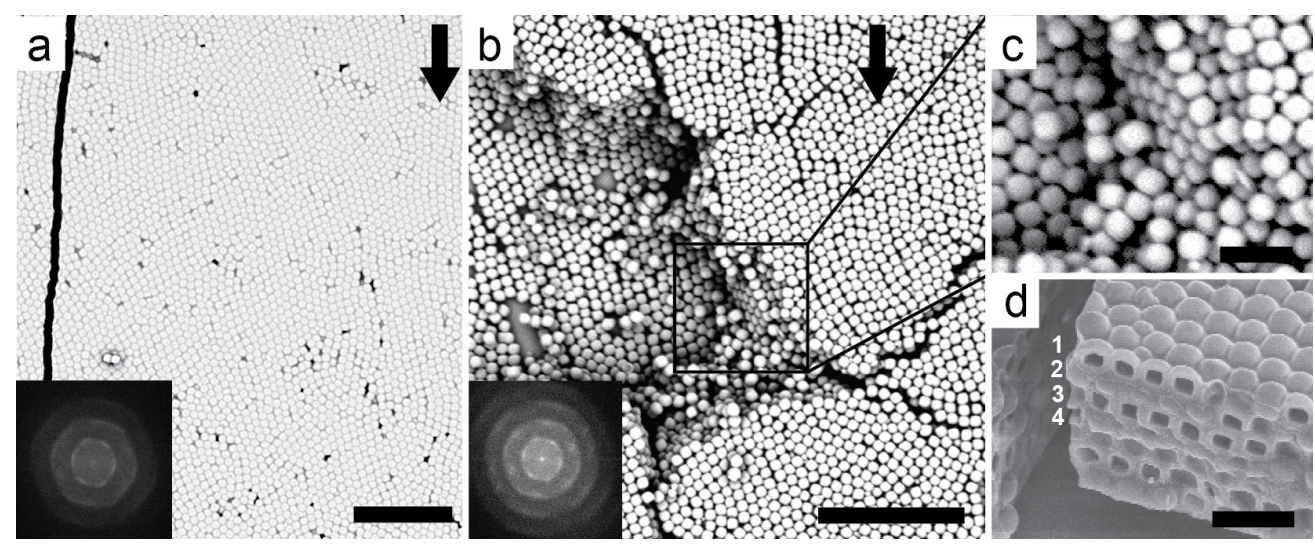

Figure 6.4. SEM image of multiple layered crystalline cube arrays of; a) an infiltrated region and b) a non-infiltrated region. For both images their corresponding Fourier transforms are shown in the insets, arrows indicate the growth direction during VD. c) Higher magnification of the side of the layers showing the location of cubes on top of niche formed by neighboring cubes in the layer below. d) Fracture cross section of infiltrated region. Scale bars are a,b) $10 \mu \mathrm{m}$ c,d) $5 \mu \mathrm{m}$.

$\Delta T$-sample, of the ordered top layer of an infiltrated crystal (Fig.6.4.a) and a fracture through multi-layered crystals (Fig.6.4.b). The Fourier transform of the images (inserts Fig. 6.4.a,b) reveal that the local cube positions are predominantly distorted hexagonal and have the same orientation. In Figure 6.4.c the stacking of the cubes can be seen more clearly. Although the image is taken without tilting the sample, cubes in all layers are visible. Evidently, the lateral positions of the cubes in a layer are slightly shifted. Furthermore, the cubes are located in the niche between two neighboring rows of cubes below, and on top of the niche between two cubes in these rows. Figure 6.4.d shows a straight fracture through an infiltrated part of the sample, revealing the square hollow-inside of the cubes. Interestingly, directly beside the crack the hollow-inside of the cubes is only visible in the top and third layer of the structure, in contrast to the second and fourth layer, where the edge of the cubes are visible. Clearly, the cubes are located in a niche formed by three cubes in the layer below, a so-called hollow-site stacking, which is characteristic for hexagonally close packed (HCP) and face centered cubic (FCC) structures of spheres.

For the SAXS measurements, the substrate was positioned perpendicular to the beam and multiple scans were performed, covering the complete area of the film. In Figure 6.5 three 2D-SAXS scattering patterns of the infiltrated top (Fig. 6.5.a) and bottom (Fig. 6.5.c) and, noninfiltrated middle (Fig. 6.5.b) regions of the $\Delta T$-sample are shown. The dark rounded bar visible to the side is the shadow of the beam stop, which protects the CCD-camera from the direct $\mathrm{X}$-ray beam. The patterns of the top and middle part of the sample both show Bragg peaks at low $q$-values with hexagonal order, which are slightly elongated along the growth direction. At higher $q$-values ring-like reflections are visible that are attributed to the form factor $P(q)$. Despite the difference in the two hexagonal pattern intensities, both were found to actually 

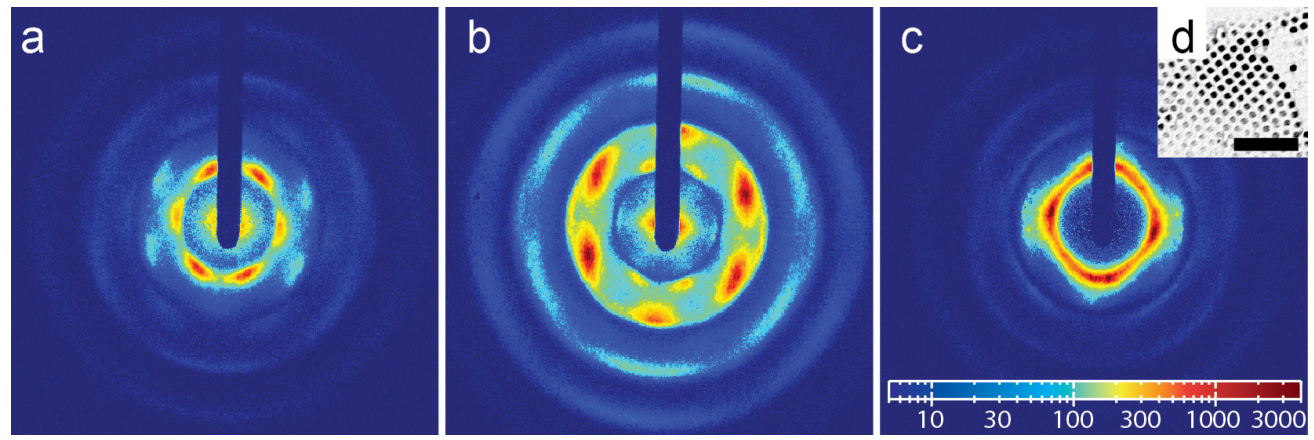

Figure 6.5. a-c) SAXS patterns of the $\Delta T$-sample taken at different positions in the film. a) top of the film, with infiltration matrix b) middle, where the particles are separated by air and c) bottom, where a second infiltration matrix was found to be present between the cubes, due to a temporary break in deposition. The vertical growth direction is aligned top to bottom. d) SEM image of simple cubic ordering in the infiltrated region where the hollow cube inside is visible. Scale bar is $5 \mu \mathrm{m}$.

originate from the same crystal structure. The total diffracted intensity, $I(q)$ is proportional to the product of both the form factor, $P(q)$, originating from particle size and shape and the structure factor, $S(q)$, arising from the periodic lattice of a crystal as given by Eq. (1.4.). Due to the connecting matrix between the cubes in the top part $P(q)$ arises from the hollow cubes centers while in the middle region $P(q)$ arises from cubic silica shells. As a result the $S(q)$ peaks are extinct or emphasized at different $q$. See appendix A.6 for a detailed analysis of the different $P(q)$ contributions.

The inner $q$-values of the Bragg peaks are $q=8.01 \mu \mathrm{m}^{-1}$ in the horizontal direction and $q=9.17$ $\mu \mathrm{m}^{-1}$ for the two vertical peaks, with a ratio of 1.12 . This corresponds to a hexagonal-type crystal with two lattice dimensions of $784 \mathrm{~nm}$ and $685 \mathrm{~nm}$. The radial width of the peaks indicates that the crystal domain size extends over about 10 particle diameters. ${ }^{[22]}$ The azimuthal width of the peaks shows that the crystal grain orientation fluctuates but the hexagonal order is directed along the growth direction. The presence of the second order Bragg peaks indicates that the crystal ordering is quite well established but decay of $P(q)$ and positional correlation fluctuations decrease the intensity of the higher orders. The higher order peaks are also influenced by the number of layers of the crystal, which is was found to be slightly higher in the middle region without a matrix compared to the top region, nine layers compared to seven layers.

Figure 6.5.c shows a diffraction pattern of the second infiltrated region about $3.5 \mathrm{~mm}$ above the film-bottom. A SEM image of the same region, close to a scratch is shown in Figure 6.5.d. Here the structure has been broken along the crystal layer parallel to the substrate, showing the simple-cubic ordering of the hollow cube interior. The pattern shows distinct peaks in the horizontal direction at $q=8.78 \mu \mathrm{m}^{-1}$ and in the vertical direction $q=9.42 \mu \mathrm{m}^{-1}$, corresponding to a simple-cubic structure with lattice distances of $716 \mathrm{~nm}$ and $667 \mathrm{~nm}$, respectively. Compared to the first infiltrated region (Figure 6.5.a), it is clear that a decrease of $70 \mathrm{~nm}$ in cube size has occurred. Closer analysis of peaks reveals that the angle between the right horizontal peak 
and the vertical peak is $85^{\circ}$ in contrast to the expected angle of $90^{\circ}$ for a square structure. Also the location of the horizontal peaks has rotated $5^{\circ}$ with respect to the hexagonal pattern. The absence of second order peaks is due to the hollow cube form factor but also because this part of the sample does not possess true long-range order. Evidently, a different structure has been formed in the bottom part of the sample. This could be cause by the difference in wetting angle and silica infiltration, as discussed earlier or by a slight change in the cube shape which might promote the formation of a $\Lambda_{1}$-lattice ${ }^{[10]}$.

\subsection{Discussion}

\subsubsection{Crystal structure and formation}

Crystal symmetries are determined by the shape of the building blocks as well as the VD preparation method. In the VD method the deposition starts at the thin solvent layer that is formed on the substrate. When the solvent layer has a thickness smaller than the cube edge length, strong capillary forces will act upon the cubes, so-called immersion forces ${ }^{[5]}$. The immersion forces will induce a flat orientation of the cubes on the substrate and induce longrange attraction between the cubes, resulting in dense packings. For single layers of cubes, distorted square to hexagonally coordinated arrays, with the latter being more dominant, were observed. Interestingly, these arrangements can be simply explained by the rounded square shape of the flat lying cubes. Jiao et al. ${ }^{[10]}$ showed that for superdisks, the $2 \mathrm{D}$ analog of superballs, two different types of dense lattice packings can be formed. The $\Lambda_{0}$-lattice, with hexagonal arrangement for more sphere-like shapes and the $\Lambda_{1}$-lattice, with distorted square arrangement for cubes with rounded edges, as shown in Figure 6.6.a. For the $\Lambda_{1}$-lattice a denser packing is achieved due to the touching cube corners, resulting in a distorted (2D) simple cubic arrangement. Jiao et al. ${ }^{[10]}$ also showed that at $m^{*}=2.572$ the $\Lambda_{0}$ and $\Lambda_{1}$ lattices are equally favorable. The cubes used in our study were found to be described by the shape parameter $m=$ 2.9 , which is very close to this value and might explain the presence of both structures.

The typical periodic distances of the hexagonal and square arrangement can be calculated (Fig 6.6.a). For the hexagonal arrangement, three periodic distances occur; one in the horizontal direction of $D$, and two in the vertical direction of $\sqrt{ }(4 / 5) D$, as indicated by the lines through the cube centers. For the square arrangement, touching cube corners form a denser packing and result in a square structure with a $85^{\circ}$ rotation, resulting in two different periodic distances. These calculated distances do not correspond exactly to the lattice values found with the SAXS patterns (Figure 6.5). However, good agreement is found for the hexagonal lattice between the ratio of the hexagonal reciprocal Bragg peaks in the vertical and horizontal direction, $1.12 \approx$ $\sqrt{ }(5 / 4)$, which converts to $\sqrt{ }(4 / 5)$ in real space. For the cubic lattice good agreement is found in that the SAXS data shows indeed two different periodic distances with a rotation angle of $85^{\circ}$.

The main part of the $\Delta T$-sample with multi-layered structures consists of stacked hexagonal layers. In this arrangement the cubes have a so-called hollow-site stacking. Figure 6.6.b shows a schematic model of how the stacking is build up for a second and third layer of cubes. For the second layer there is no preferential position. However, for the third layer, the first possible 
a

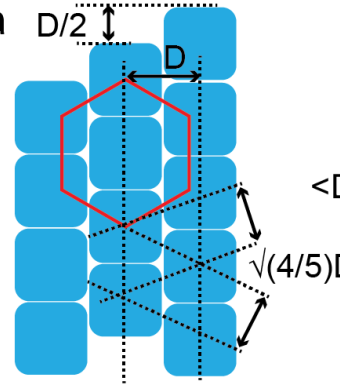

b

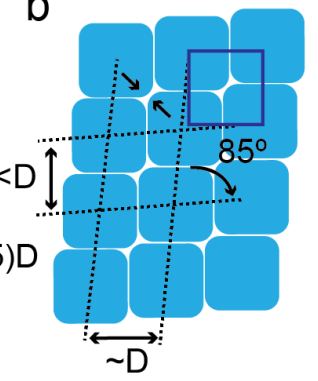

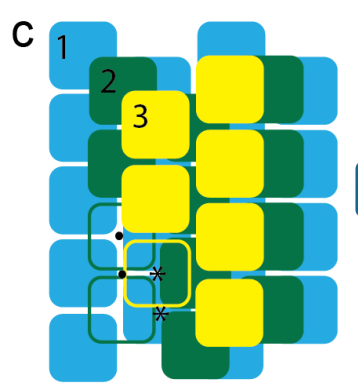

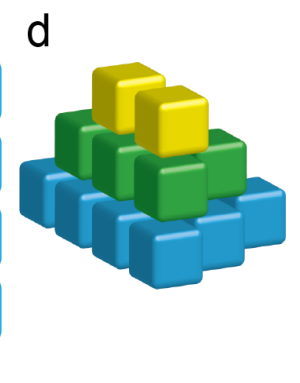

Figure 6.6. Schematic model of arranged rounded squares (superballs in 3D) in a) an hexagonal arrangement and b) square arrangement, (comparable to the $\Lambda_{0}$-lattice and $\Lambda_{1}$-lattice as in ref. [I0], with their periodic lattice distances. c) Possible locations for the second (dots) and third (asterisk) layer in a hexagonal arrangement and d) representation of the formed 3D lattice.

location is above a niche in both layers and the second is located above a particle in the first layer. From the sideward stacking observed in Figure 6.4.c, it is clear that the third layer is positioned above the first niche and not a particle in the first layer. Figure 6.6.c shows a 3D representation of the resulting structure. The specific location of the particles could be a result of growth at a particular crystal plane during the VD, which is known to occur for thin crystals of spheres, inducing FCC stacking ${ }^{[23,24]}$. However, the growth mechanism of the specific FCC structure during the deposition of thick (>5 layers) crystals of spheres is still under investigation. ${ }^{[23,25]}$ So, in order to understand the formation mechanism of multi-layered cube structures, more investigation is needed.

\subsubsection{Crystal Defects}

In the formed crystals, different defects were observed, such as cracks, vacancies and imperfect crystal structures, which affect the crystal quality. The small cracks in the crystals are common defects observed in crystals created with $\mathrm{VD}^{[5]}$. Due to tensile stresses during the drying step, the distances between two particles decreases and cracks will form. In the SEM images additional shrinkage is caused by the exposure to electrons and the high vacuum in the SEM ${ }^{[5]}$. Vacancies in the crystalline structure will occur when the evaporation speed of the solvent is faster than the influx of cubes. The fast solvent evaporation also causes the imperfect crystal structures as the cubes do not have enough time to move to the optimal lattice position before they are pinned down to the substrate. In order to gain more control over the formed structures and possibly prevent defects, the VD conditions should be optimized and well controlled.

\subsubsection{Crystal Infiltration}

The silica matrix observed between the cubes at the top of the film, can be explained by the etching of silica by aqueous solutions at elevated temperatures: amorphous silica, $\mathrm{SiO}_{2}$, partly dissolves in water as silicic acid, $\mathrm{Si}(\mathrm{OH})_{4}{ }^{\left[{ }^{[26]}\right.}$ which will precipitate in between the cubes during 
deposition, creating a $\mathrm{SiO}_{2}$ matrix embedding the cubes (Fig. 6.3). With the $\Delta T$-setup the cube dispersion is heated to $55^{\circ} \mathrm{C}$, which promotes silica dissolution. In addition, the deposition does not start immediately when using the $\Delta T$-setup, because, in contrast to the oven-setup, evaporation is slower due to the cooling at the surface in the $\Delta T$-setup. This additional time allows the concentration of soluble silica to increase over a longer time period and explains the presence of large infiltration regions observed in samples made with the $\Delta T$-setup. The fact that the infiltration is only located at the top of the film indicates that at a certain point the $\mathrm{Si}(\mathrm{OH})_{4}$ concentration has decreased such that the inter-particle voids cannot be completely filled anymore. Apparently, the concentration of soluble silica is not constant over time. The concentration decrease can be caused by the precipitation in the form of $\mathrm{SiO}_{2}$ between the cubes during the deposition of the cubes, which also means a decrease in the source of the $\mathrm{Si}(\mathrm{OH})_{4}$.

Dissolution of particles is on the one hand not desirable because when the cubes are etched they become smaller and eventually will lose their shape. On the other hand, a silica matrix also has advantages. Firstly, it stabilizes the crystal structures by 'glueing' particles together, preventing fracture, which has been observed before in infiltrated crystals of silica spheres ${ }^{[27]}$. The presented mechanism has already been employed for the improvement of photonic crystals of spheres fabricated with the vertical deposition technique. ${ }^{[28,29]}$ Secondly, the silica matrix also seems to be the cause of the stabilization of the simple cubic lattice in the bottom part of the sample.

\subsection{Conclusions}

Using the VD technique large crystalline films of ordered monodisperse hollow colloidal cubes can be prepared in a controlled way. During the deposition, capillary forces and convective flow induce the self-assembly and the cube orientation was found to be influenced by the flat substrate and neighboring cubes. Due to the high temperatures used in the experiments, the amorphous silica slightly dissolved and precipitated again in the close-packed structures of the cubes forming a connecting matrix between the cubes.

In monolayers the cubes ordered into both hexagonal and square arrangements similar to the $\Lambda_{0}$ and $\Lambda_{1}$ lattices as predicted for dense $2 \mathrm{D}$ packings of superballs ${ }^{[10]}$. That both structures are present is related to the fact that the shape parameter of the cubes of $m=2.9$ is very close to the $m^{*}=2.572$ at which both are equally likely to form. Furthermore, the formation mechanism is governed by capillary forces and convective flow, which influence the structure formation. In multi-layered structures the layers were found to possess two different types of arrangements, The first structure consisted of layers with in-plane hexagonal close-packing and a hollow-site stacking, similar to that of FCC for spheres, was most dominant throughout the sample. The second structure was a simple cubic lattice that only formed at the bottom of the sample and seemed to be stabilized by the presence of the silica matrix. 


\section{Acknowledgements}

This work is in part the result of the bachelor thesis project of Fabian Hagemans and collaboration with Laura Rossi. Sonja Castillo is thanked for the synthesis of the hematite cubes. Dima Byelov is thanked for help with the SAXS data acquisition and together with Jan Hilhorst for many fruitful discussions. The European Synchrotron Radiation Facility (ESRF) in Grenoble is acknowledged for the provided beam-time. A. Snigirev, I. Snigireva, C. Detlefs and T. Roth from ID06 are thanked for the collaboration and support during the measurements. Hans Meeldijk and Chris Schneijdenberg are thanked for their assistance with the electron microscopes.

\section{Appendix}

\section{A.6. The effect of the form factor in the diffraction patterns.}

In Figure 6.10.a and 6.10.b the radial averaged scattering intensity profiles of Figure 6.5.a and 6.5.b are displayed on linear and log scale. It can be seen that at low $q$-values the maximum the Bragg peaks (or $S(q)$ ) in the top overlaps with a minimum in intensity of the middle region. The opposite occurs for the second Bragg peaks. Both first minima in intensity were determined at $q_{\text {min }}=14.92 \mu \mathrm{m}^{-1}$ for the infiltrated region and at $q_{\text {min }}=8.85 \mu \mathrm{m}^{-1}$ for the separate cubes (arrows Figure 6.10).

The difference in $P(q)$ in the patters is caused by the presence of hollow air cubes in a silica matrix in the top region versus hollow cubic shells in the middle region. The cubes have a superball shape with $m=3$ which is close to that of a sphere with $m=2$. Moreover, the cubes all lie with one flat side on the substrate, which is perpendicular to the beam. Therefore, we approximate the form factor of these rounded cubes with that of a sphere; ${ }^{[30]}$

$$
P_{\text {sphere }}(q, R)=\left[3 \frac{\sin (q R)-q R \cos (q R)}{(q R)^{3}}\right]^{2}
$$

where the sphere radius, $R$ is approximated as $(D+L) / 4$ and $q$ is the wave vector. For the silica shell, $P(q)$ is approximated as;

$$
P_{\text {shell }}=\frac{1}{V_{\text {shell }}}\left[V_{\text {total }} P_{\text {sphere }}\left(q, R_{2}\right)-V_{\text {inner }} P_{\text {sphere }}\left(q, R_{1}\right)\right]^{2}
$$

where $R_{2}$ is the outer radius of the shell, $V_{\text {total }}$ is the volume of a the outer sphere with radius $d$, $V_{\text {inner }}$ is the volume of the inner sphere with radius $R_{1}$, and $V_{\text {shell }}=V_{\text {outer }}-V_{\text {inner }}$, the volume of the shell.

Despite the simplicity of the form factors they are able to clarify the difference in features observed in the scattering data (Fig. 6.7.b). For instance, the form factor minima can be clearly identified in the averaged radial intensity profiles as indicated with the arrows. The different $P(q)$ furthermore explain the intensity difference of the Bragg spots in both patterns, which are located at corresponding $q$-values. From this we conclude that both regions have the same crystal structure, with $S(q)_{\text {top }}=S(q)_{\text {middle }}$ emphasized differently by $P(q)$. 

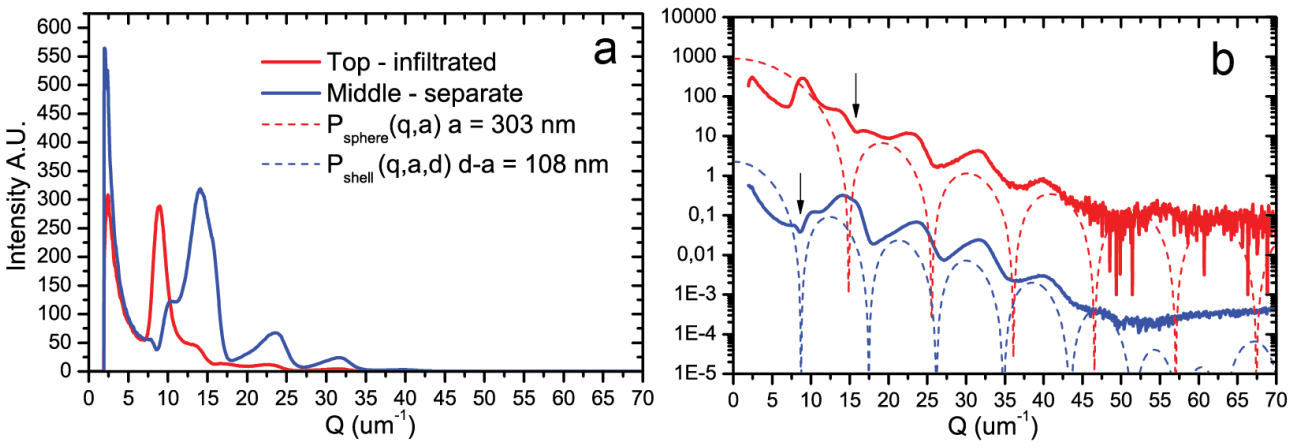

Figure 6.7. Radial average of 2D SAXS patterns of the infiltrated top region (red) and the non-infiltrated middle (blue) region of the $\Delta \mathrm{T}$-sample on a) linear scale and b) on log scale fit. $\ln \mathrm{b}$ ) the blue curve is off-set in intensity by factor $10^{3}$ for clarity. The curves are fitted with a form factor of a homogeneous sphere and a spherical shell, respectively. Arrows indicate the first $P(q)$ minimum.

\section{References}

1. P. Jiang, J. F. Bertone, K. S. Hwang and V. L. Colvin, Chem. Mater., 1999, 11, 2132-2140.

2. Y. Vlasov, X. Z. Bo, J. C. Sturm and D. J. Norris, Nature, 2001, 414, 289-93.

3. Z. Zhou and X. S. Zhao, Langmuir, 2004, 20, 1524-6.

4. J. Zhang, Y. Li, X. Zhang and B. Yang, Adv. Mater., 2010, 22, 4249-69.

5. N. Denkov, O. Velev, P. Kralchevski, I. Ivanov, H. Yoshimura and K. Nagayama, Langmuir, 1992, 8, 3183-3190.

6. J. Zhang, Z. Sun and B. Yang, Curr. Opin. Colloid Interface Sci., 2009, 14, 103-114.

7. Z. Zhou and X. S. Zhao, Langmuir, 2005, 21, 4717-23.

8. V. R. Dugyala, S. V. Daware and M. G. Basavaraj, Soft Matter, 2013, 9, 6711-6725.

9. L. Rossi, S. Sacanna, W. T. M. Irvine, P. M. Chaikin, D. J. Pine and A. P. Philipse, Soft Matter, 2011, 7, 4139-4139.

10. Y. Jiao, F. H. Stillinger and S. Torquato, Phys. Rev. Lett., 2008, 100, 245504.

11. Y. Jiao, F. Stillinger and S. Torquato, Phys. Rev. E, 2009, 79, 1-12.

12. T. Sugimoto and K. Sakata, J. Colloid Interface Sci., 1992, 152, 587-590.

13. T. Sugimoto, M. M. Khan, A. Muramatsu and H. Itoh, Colloids Surf. A., 1993, 79, 233-247.

14. L. Rossi, S. Sacanna, W. T. M. Irvine, P. M. Chaikin, D. J. Pine and A. P. Philipse, Soft Matter, 2011, 7, 4139-4142.

15. C. Graf, D. L. J. Vossen, A. Imhof and A. van Blaaderen, Langmuir, 2003, 19, 6693-6700.

16. Y. Jiao, F. H. Stillinger and S. Torquato, Phys. Rev. E, 2010, 81, 1-8.

17. A. Snigirev, V. Kohn, I. Snigireva and B. Lengeler, Nature, 1996, 384, 49-51.

18. V. Kohn, I. Snigireva and A. Snigirev, Opt. Commun., 2003, 216, 247-260.

19. M. Drakopoulos, A. Snigirev, I. Snigireva and J. Schilling, Appl. Phys. Lett., 2005, 86, 014102.

20. B. Lengeler, C. Schroer, J. Tummler, B. Benner, M. Richwin, A. Snigirev, I. Snigireva and M. Drakopoulos, J. Synchrotron Rad., 1999, 6, 1153-1167.

21. J. Marqués-Hueso and H. J. Schöpe, Progr Colloid Polym Sci, 2008, 48-56.

22. A. Guinier, "X-Ray Diffraction in Crystal, Imperfect Crystals, and Amorphous Bodies", Dover, New York, 1994.

23. L. Meng, H. Wei, A. Nagel, B. J. Wiley, L. E. Scriven and D. J. Norris, Nano Lett., 2006, 6, 2249-53.

24. D. J. Norris, E. G. Arlinghaus, L. Meng, R. Heiny and L. E. Scriven, Adv. Mater., 2004, 16, 1393-1399.

25. D. D. Brewer, J. Allen, M. R. Miller, J. M. D. Santos, S. Kumar, D. J. Norris, M. Tsapatsis and L. E. Scriven, Langmuir, 2008.

26. G. B. Alexander, W. M. Heston and R. K. Iler, J. Phys. Chem, 1954, 6, 453-455.

27. A. P. Philipse, J. Mater. Sci. Lett., 1989, 8, 1371-1373.

28. B. Hatton, L. Mishchenko, S. Davis, K. H. Sandhage and J. Aizenberg, Proc. Natl. Acad. Sci. U. S. A., 2010, 107, 10354-9.

29. L. Wang and X. S. Zhao, J. Phys. Chem. C, 2007, 111, 8538-8542.

30. J. S. Pedersen,"Modeling of Small-Angle Scattering Data from Colloids and Polymer Systems. “, in "Neutrons, X-rays and Light: Scattering Methods Applied to Soft Condensed Matter", ed. P. Linder and T. Zemb, Elsevier Science B.V., 2002, pp.391-420. 

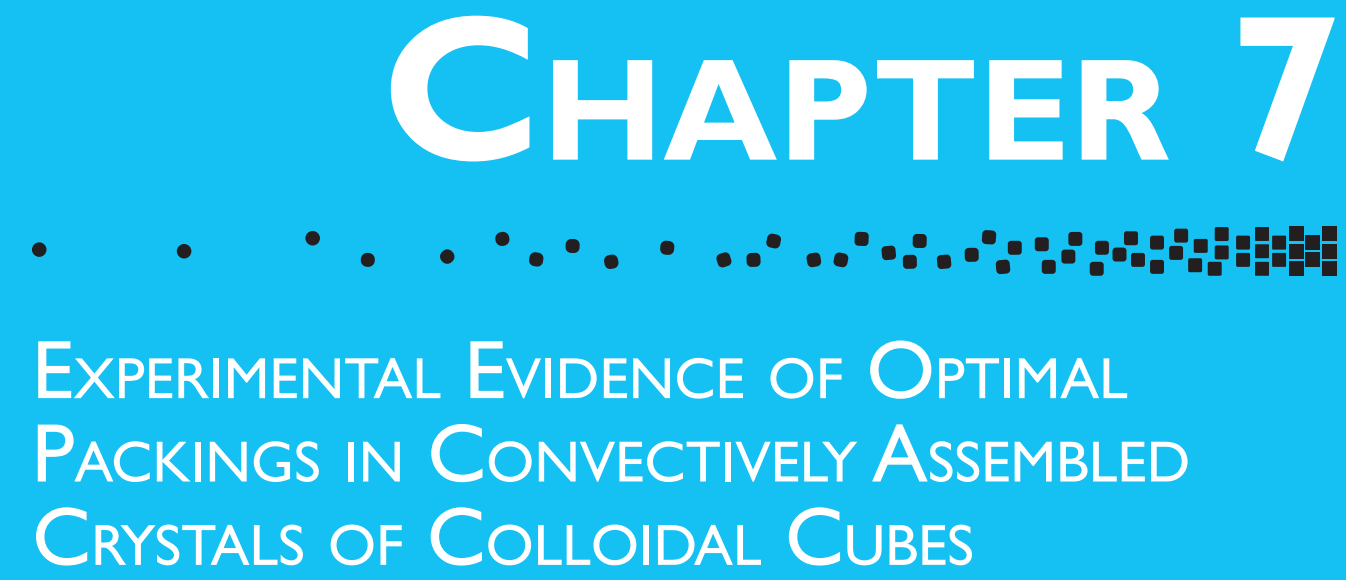

Abstract

Simulations of optimal two-dimensional packings of colloidal superballs of various shapes predict that, particularly for particles with sufficiently sharp edges, a rhombic lattice provides the densest packing. To investigate this prediction we study hollow silica cube structures formed via two different convective assembly techniques: horizontal deposition and flow controlled vertical deposition. With optical microscopy the formation of the structures is studied and with scanning electron microscopy the local order of the cubes is analyzed. The analysis reveals that both the hexagonal $\Lambda_{0}$-lattice and rhombic $\Lambda_{1}$-lattice are formed by the cubes with a larger excess of the $\Lambda_{1}$-lattice as predicted by the simulations. 


\section{I. Introduction}

In Chapter 6 it has been shown that hollow colloidal cubes can assemble into close-packed single layer and multilayered arrangements via convective assembly as described in Chapter1, Section 1.3. These colloidal cubes can be considered as superballs, that are described by ${ }^{[1,2]}$ :

$$
\left|\frac{x}{a}\right|^{m}+\left|\frac{y}{a}\right|^{m}+\left|\frac{z}{a}\right|^{m} \leq 1
$$

where $m$ is the shape parameter, which indicates the extent of deformation from a sphere $(m=2)$ to a perfect cube $(m \rightarrow \infty)$ and $a$ is half the edge length $D$.

Molecular dynamics simulation of two-dimensional (2D) packings of the superballs predict that the densest packings are the $\Lambda_{0}$ - and $\Lambda_{1}$-lattices ${ }^{[3]}$. The maximum packing densities of the lattices increases rapidly as $m$ increases from $2 \rightarrow \infty$ and for $m>m^{*}=2.572$ the packing density is found to be highest for the $\Lambda_{1}$-lattice. Recent experiments and simulations of the phase behavior of rounded squares in $2 \mathrm{D}$ have shown that a rhombic phase will form at high volume fractions ${ }^{[4,5]}$. At maximum packing density this rhombic structure is similar to the $\Lambda_{1}$-lattice. Because with convective assembly methods close-packed structures of the cubes are obtained, it is expected that the $\Lambda_{1}$-lattice will be realized for cubes with higher $m$-values. However, convective assembly does not occur under equilibrium conditions and it is of interest to see what the effect of a larger $m$-value on the close-packed structures is.

Experimentally the shape of the hollow silica cubes can be controlled by using larger template hematite cube of which $m$ is known to increase with size ${ }^{[6]}$ (see also Chapter 5, Section 5.3.2). However, an increase in size also entails an increase in sedimentation speed which makes vertical deposition an unsuitable technique because particles will sediment and little deposition will occur. Various convective assembly methods exist that are specially designed to counteract the gravitational effects ${ }^{[7-11]}$. One of these, the convective transport due to addition of a temperature gradient to the suspension ${ }^{[7]}$ was explored in the previous chapter for small cubes (edge length $D=774 \mathrm{~nm}$ ) and resulted in large crystalline areas. This technique, however, was shown to have an etching effect on the hollow silica cubes. Another solution is rotating the setup to a horizontal position ${ }^{[12,13]}$. In this way sedimentation of the cubes actually causes an increase in particle concentration close to the substrate and makes transport to the growth front faster ${ }^{[14]}$. In addition, the horizontal methods allows working at lower temperatures and the previously mentioned etching effect can be avoided. A third way to prevent sedimentation is to increase the evaporation speed and meniscus movement by slowly removing the suspension from the dispersion container, a technique called flow controlled vertical deposition ${ }^{[8,15]}$.

Here we employ two different convective assembly techniques, horizontal deposition and flow controlled vertical deposition for the convective assembly of larger hollow colloidal silica cubes. Their assembly, structure formation and resulting deposits are studied with optical and scanning electron microscopy. The primary aim here is to assess whether the predicted $\Lambda_{0}$ - and $\Lambda_{1}$-lattices indeed occur in the silica cubes packings. 


\subsection{Experimental}

\subsection{Colloidal cubes}

The precursor hematite particles used for the preparation of the hollow silica cubes were prepared according to the procedure described in Chapter 5, Section 5.2. For these specific precursor particles 50.53 $\mathrm{g} \mathrm{FeCl}_{3} .6 \mathrm{H}_{2} \mathrm{O}$ (Sigma-Aldrich, 99\%) dissolved in $100 \mathrm{~mL}$ water was mixed quickly (20 s) with $20.18 \mathrm{~g} \mathrm{NaOH}$ dissolved in $100 \mathrm{~mL}$ water under vigorous magnetic stirring. The mixing was followed by aging the suspension at $100{ }^{\circ} \mathrm{C}$ for 8 days. After repeated washing with water by centrifugation hematite cubes were obtained as shown in Figure 7.1.a. Water from a Millipore system was always used.

The hollow silica cubes were prepared by mixing $75 \mathrm{~mL}$ of $4.7 \mathrm{wt} \% \mathrm{PVP}-40$-functionalized hematite cubes in ethanol with $800 \mathrm{~mL}$ ethanol (Interchem), 15mL 1 wt\% TMAH (Fluka) solution and $122 \mathrm{~mL}$ water. To this mixture $20 \mathrm{~mL}$ TEOS (Sigma-Aldrich) was added drop wise over the course of $\sim 3$ hours under mechanical stirring and sonication. The hematite core was removed by dispersing the cubes in $5 \mathrm{M} \mathrm{HCl}$ (Merck) followed by repeated washing by centrifugation with water followed by ethanol. The obtained hollow cubes were stored in ethanol to prevent silica etching. A typical TEM image of the obtained hollow silica cubes is shown in Figure 7.1.b.

The average edge length $\left\langle D>\right.$, the polydispersity $\sigma_{D}$, average silica shell thickness $\left\langle t_{\mathrm{SiO}_{2}}>\right.$ and the deformation parameter $m$ of the cubes were determined with transmission microscopy (TEM, TECNAI 10 or 12) of at least 100 images. The specific properties of both precursor and
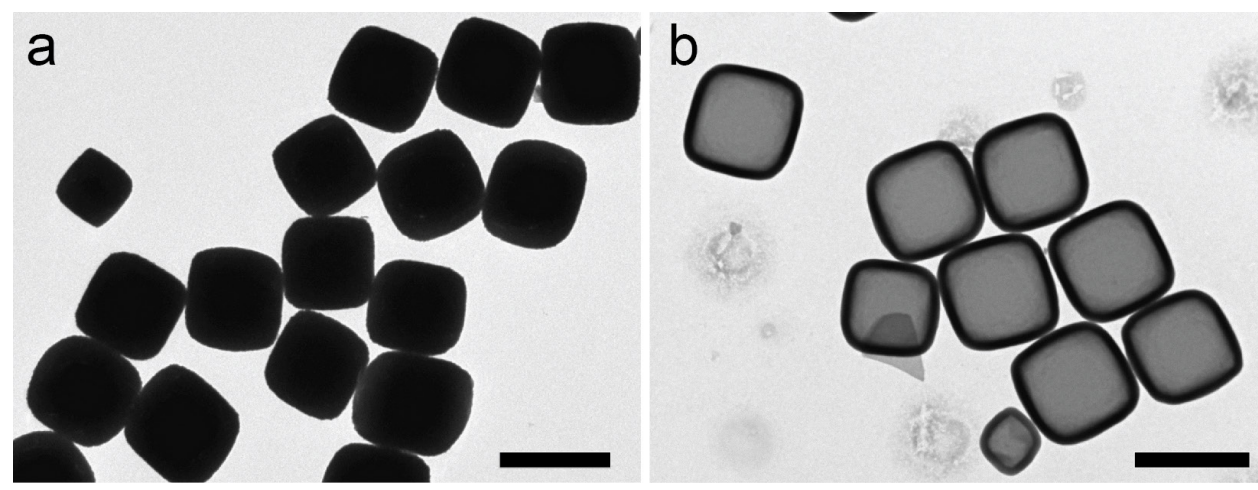

Figure 7.I. TEM images of a) the precursor hematite particles $\mathrm{HC5}$ and b) hollow silica cubes $\mathrm{HC5}_{-}$ Si_H. Scale bars are I $\mu \mathrm{m}$.

Table 7.I. Characteristics of the precursor hematite cubes and hollow silica cubes.

\begin{tabular}{l|ccccc}
\hline Sample & Composition & $\begin{array}{c}<\mathbf{D}> \\
\mathbf{( n m})\end{array}$ & $\begin{array}{c}\sigma_{\mathbf{D}} \\
(\mathbf{n m})\end{array}$ & $\begin{array}{c}<\mathbf{t}_{\mathrm{SiO} 2}> \\
(\mathbf{n m})\end{array}$ & $m$ \\
\hline HC5 & Hematite & 932 & 5.5 & - & - \\
HC5_Si_H & Hollow Silica & 1028 & 3.5 & 48 & 3.55 \\
\hline
\end{tabular}


hollow cubes are shown in Table 7.1. The hollow silica cubes in this study were found to have shape parameter $m=3.55$, which is a clear increase compared to the smaller hollow silica cubes used in Chapter 6 for which $m=2$.9. This increase allows us to study the effect of $m$ on the convective assembled structures.

\subsubsection{Sample Preparation}

Prior to convective assembly experiments a stock dispersion of hollow silica cubes in water was prepared by repeated centrifugation. The volume fraction of this stock dispersion was determined as described in Chapter 6, Section 6.2.2 and the desired volume fractions were achieved by diluting the stock dispersions with the appropriate amount of water. Typically, cube dispersions with a volume fraction in the range of 0.1-1.2 $\mathrm{v} \%$ in water were used.

Microscope glass slides with dimensions of $24 \times 50 \times 0.15 \mathrm{~mm}$ (Menzel-Glaser \#1) were etched with a $7 \mathrm{wt} \% \mathrm{KOH}$ (Merck) aqueous solution in ethanol for at least 1 hour but usually 16 hours. Subsequently, the slides were rinsed thoroughly with ethanol and water and dried in air. If required the slides were cut to smaller sizes using a diamond pen prior to the experiments.

\subsubsection{Convective assembly experiments \\ Horizontal deposition}

Two different horizontal depositions methods were used as shown in Figure 7.2.a and 7.2.b. The first consisted of placing of a small droplet on a microscope slide and is further referred to as a drying droplet (DD) method. The second consisted of two microscope slides cut to $10 \times 50$ $\mathrm{mm}$ with the first slide placed horizontal and the second slide placed with an angle of $25-35^{\circ}$ on top of the first. A droplet was placed into the corner formed by the two slides. This method will be further referred to as the horizontal deposition (HD) method. In both methods cubes will be pinned by the meniscus and due to convective flow the cubes will be transported to the growth front, as schematically shown in Figure 7.2.c.

A DD experiment was performed by dropping $25 \mu \mathrm{L}$ dispersion from $3 \mathrm{~cm}$ height onto the slide, this spreads out the droplet and reduces the solvent film thickness. To further decrease the contact angle half of the volume of the dispersion was removed after $\sim 30$ s. It was empirically found that after this time the meniscus is pinned at the edges of the droplet. A HD experiment typically consisted of placing $100 \mu \mathrm{L}$ dispersion in the corner of the slides and covering the slides with a $2 \mathrm{~L}$ crystallization dish to avoid irregular air flow. Slow solvent evaporation occurred over 8 hours at room temperature.

\section{Flow controlled vertical deposition}

The flow controlled vertical deposition (FCVD) method was based on ref ${ }^{[8]}$ and consisted of a substrate of $10 \times 37 \mathrm{~mm}$ placed into a glass flask of $50 \mathrm{~mm}$ in height and $20 \mathrm{~mm}$ in diameter at an angle of approximately $10^{\circ}$. This flask has a small nozzle at the bottom that can be connected to a peristaltic pump (Miniplus 3, Gilson) to control the surface dropping velocity, $v_{d}$. The flask was heated with a cylindrical heating block that was temperature controlled by a thermostatic 
a Drying Droplet

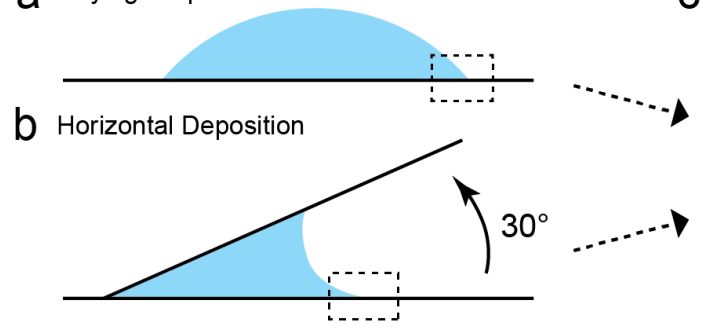

d Flow Controlled Vertical Deposition

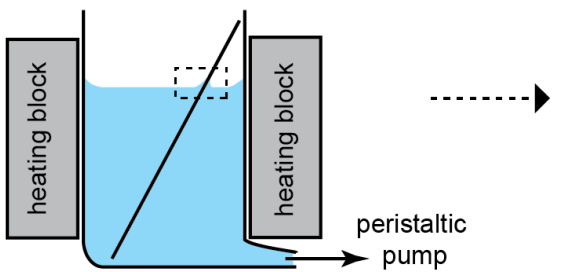

C

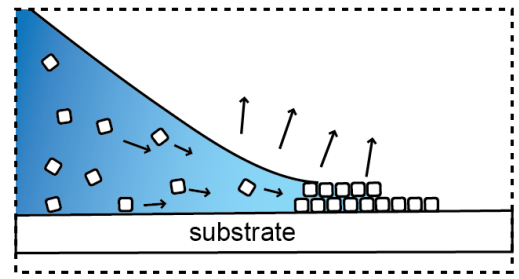

e

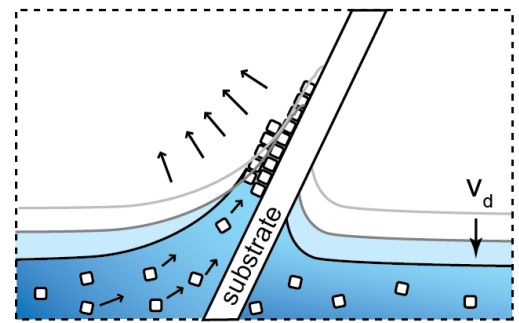

Figure 7.2. Schematic representation of the convective assembly methods. a) Drying droplet (DD) method b) Horizontal deposition (HD) method in which a second slide is position on top of the substrate forming a wedge-shaped. c) Assembly of cubes due to solvent evaporation at the horizontal substrate. d) Flow controlled vertical deposition (FCVD) method. e) Assembly of cubes on the titled substrate where the meniscus is stretched due to the solvent dropping velocity, $v_{d}$, and faster evaporation is induced.

water bath. A schematic representation is shown in Figure 7.2.d. Experiments were performed with $8-20 \mathrm{~mL} 0.5-2 \mathrm{v} \%$ cube dispersions at $45^{\circ} \mathrm{C}$, with $v_{d}=1 \mathrm{~mm} / \mathrm{h}$ with a duration of $24-40$ hours. Due to evaporation of the solvent, particles were transported to the solvent-air meniscus and pinned on the substrate, as shown in Figure 7.2.e.

\subsubsection{Characterization \\ Optical Microscopy}

Colloidal cubes in dispersion were imaged on a single particle level with a Nikon ECLIPSE Ti-U inverse optical microscope, equipped with a $100 \times$ Nikon Plan Ako VC Oil immersion and CCD camera. Drying droplet samples were transferred to the microscope immediately after the removal of the excess dispersion.

\section{Scanning electron microscopy}

The deposits obtained from the three convective assembly methods were imaged with scanning electron microscopy (SEM) using a Phenom FEI microscope. The samples were connected with carbon tape to a SEM specimen stub and coated with a 6-8 nm layer of platinum using a sputter coater. To reveal the thickness, the samples were scratched with a razor blade. The particle centers and rotation $a$ with respect to the $x$-axis (as shown in Figure 7.3.a) of the images were retrieved from the SEM images using analySIS ${ }^{\circledR}$ iTEM software. 


\section{Data analysis}

Analysis of the nearest neighbor positions and lattice identification were performed with IDL routines (Interactive Data Language, Research Systems, Inc.; http://www.exelisvis.com (accessed July 2014)).

We can identify three lattices that the cubes can assemble into, the $\Lambda_{0}, \Lambda_{1}$ and the square lattice. For these three lattices we defined criteria based on the cube orientation on the lattice positions. The lattice vectors of $\Lambda_{0}$ and $\Lambda_{1}$ are given by ${ }^{[3]}$ :

$$
\begin{array}{ll}
\Lambda_{0} \text {-lattice: } & \mathbf{e}_{1}=D \mathbf{i}, \\
& \mathbf{e}_{2}=(D / 2) \mathbf{i}+(D / 2)\left(2^{m}-1\right)^{1 / m} \mathbf{j}, \\
\Lambda_{1} \text {-Lattice: } & \mathbf{e}_{1}=(D / 2) 2^{(1-1 / m)} \mathbf{i}+(D / 2) 2^{(1-1 / m)} \mathbf{j}, \\
& \mathbf{e}_{2}=(D / 2)\left(2^{-1 / m}-2^{1 / 2} s\right) \mathbf{i}+(D / 2)\left(2^{-1 / m}+2^{1 / 2} s\right) \mathbf{j},
\end{array}
$$

where $\mathbf{i}, \mathbf{j}$ are the unit vectors along the $x$ and $y$ directions, $D$ is the particle edge length, $m$ is the deformation parameter and $s$ is the smallest positive root of the following equation:

$$
\left|2^{-(1+1 / m)}-2^{-1 / 2} s\right|^{m}+\left|2^{-(1+1 / m)}+2^{-1 / 2} s\right|^{m}=1 .
$$

For the cubes used in this study $m=3.55$ (see Table 7.1) and hence $s=0.8316795$. The corresponding lattice vectors in $x$ and $y$ expressed in terms of $D$ are given in Table 7.2. Here we also show the lattice vectors for a square lattice given by:

Square-lattice: $\quad \mathbf{e}_{1}=D \mathbf{i}$ and $\mathbf{e}_{2}=D \mathbf{j}$.

Figure 7.3.c and 7.3.d show the schematic illustration of the $\Lambda_{0}$ and $\Lambda_{1}$ lattice packings, where the center cube (blue) in both cases is surrounded by six other cubes which centers lie on a hexagon. However, the orientation of the cube with respect to these six particles is clearly different in the lattices. To illustrate this we have drawn a dashed line across the face diagonal of the center cubes, which makes an angle of $45^{\circ}$ with the $\mathrm{x}$-axis and encircled the nearest neighbors (NNs) within a radius $<1.79 D$. This shows that for the $\Lambda_{0}$-lattice there are zero NNs which are positioned on this line, while in the $\Lambda_{1}$-lattice, if we take into account that the cube has fourfold symmetry, four of the NNs are positioned on the dashed line.

Because we can extract the particle positions and the face diagonal from the images, we calculated the exact positions of the NNs in the three lattices. The angle $\theta$ they make with respect to the face diagonal of the center cube was also determined shown in Figure 7.3.b. For the $\Lambda_{0}$-lattice this revealed 2 NNs at distance $D$ with $\theta= \pm 45^{\circ}$ (red particles Fig.7.3.c) and 4 NNs

Table 7.2. Lattice vectors of the different lattice packings observed for the cubes with $m=3.55$

\begin{tabular}{l|cccc}
\hline & \multicolumn{2}{|c}{$e_{1}$} & \multicolumn{2}{c}{$e_{2}$} \\
Lattice & $x$ & $y$ & $x$ & $y$ \\
& $(D)$ & $(D)$ & $(D)$ & $(D)$ \\
\hline$\Lambda_{0}$ & 1.0 & 0.0 & 0.5 & 0.87 \\
$\Lambda_{1}$ & 0.83 & 0.83 & -0.18 & 1.0 \\
Square & 1.0 & 0.0 & 0.0 & 1.0 \\
\hline
\end{tabular}



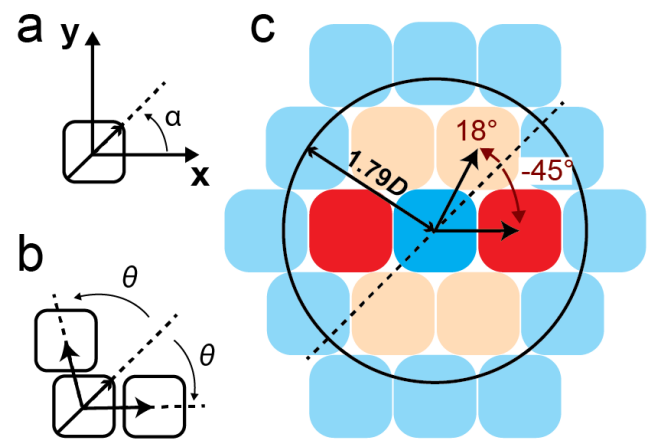

$$
\Lambda_{0} \text {-lattice }
$$

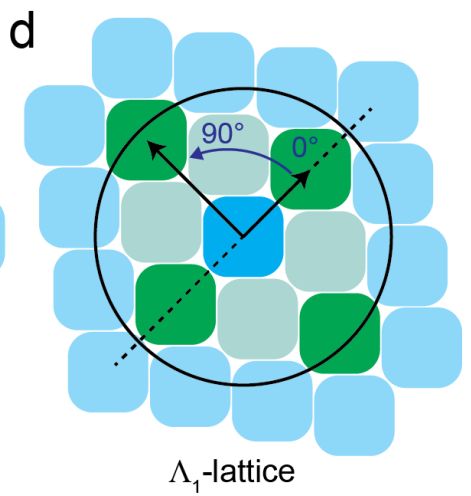

Figure 7.3. Schematic illustration of a) from the SEM images the orientation of the cubes is determined by the angle, $\alpha$, the long diagonal makes with the $x$-axis b) the angle $\theta$ the nearest neighbors make with the face diagonal of a cube and c) the $\Lambda_{0}$ and d) $\Lambda_{1}$-lattices of the cubes used in this study.

at distance $1.1 D$ with $\theta= \pm 18^{\circ}$ (orange particles Fig.7.3.c). For the $\Lambda_{1}$-lattice there are 2 NNs at $1.16 D$ with $\theta=0^{\circ}$ and $2 \mathrm{NNs}$ at $1.66 \mathrm{D}$ with $\theta=90^{\circ}$ (dark green particles Fig.7.3.d) and 4 NNs at $1.01 D$ with $\theta= \pm 35^{\circ}$ or $\pm 55^{\circ}$ (light green particles in Fig.7.3.d.). The square-lattice has of course 4 NNs at $D$ with $\theta=0^{\circ}$ or $90^{\circ}$ and 4 NNs at $\sqrt{2} D$ with $\theta= \pm 45^{\circ}$.

For each particle position the NNs were determined within a range of $1.89 \mathrm{D}$, which allows for small deviations of the NN positions that occur due to cube polydispersity and drying effects. Next, the average cube orientation $\langle\alpha\rangle$ of the NNs and the center particles was determined because the accuracy of the $\alpha$ analysis in the SEM images was found to be low and the NNs positioned on one of the lattices should have the same orientation. This was followed by the extraction of $\theta$ from the NN positions. The exact procedure is described in appendix A.7.1.

Based on the number of specific $\theta$ found for the NNs, the cube is assigned a $\Lambda_{0}$ or $\Lambda_{1}$-lattice based on the exact selection criteria shown in Table 7.3. We again compensate for the small deviations from an ideal lattice by allowing a deviation, $\sigma$, in $\theta$. In short, a $\Lambda_{1}$-lattice is assigned when $4 \mathrm{NNs}$ with $\theta \approx 0^{\circ}$ or $90^{\circ}$ are present because these $\theta$ are only realized in the $\Lambda_{1}$-lattice. The other NN angles are too similar to those of the $\Lambda_{0}$-lattice to be used for assignment. A $\Lambda_{0}$-lattice

Table 7.3. Number of $N N$ angles with specific $\theta$-value required for the assignment of the lattices.

\begin{tabular}{l|cccc}
\hline & \multicolumn{3}{|c}{$\boldsymbol{\theta}$} \\
& $0^{\circ} / \pm 90^{\circ}$ & $\pm 45^{\circ}$ & $\pm 18^{\circ}$ & $\pm 45^{\circ}$ \\
Lattice assigned & $\sigma=10^{\circ}$ & $\sigma=5^{\circ}$ & $\sigma=10^{\circ}$ & $\sigma=3^{\circ}$ \\
\hline$\Lambda_{0}$ & $\leq 2$ & 2 & 4 & \\
$\Lambda_{0}$-defect & $\leq 2$ & 2 & 3 & \\
$\Lambda_{1}$ & $\geq 4$ & & & \\
$\Lambda_{1}$-defect & 3 & & & $\geq 3$ \\
Square & $\geq 3$ & & & \\
\hline
\end{tabular}


is assigned when 4 NNs at $\theta \approx \pm 18^{\circ}$ are present and 2 NNs at $\theta \approx 45^{\circ}$, for the latter $\sigma$ has been decreased due to the proximity to the $\theta$ of the NNs present in $\Lambda_{1}$. To avoid incorrect assignment the additional criteria for the $\Lambda_{0}$-lattice assignment is that there should be $\leq 2$ NNs with $\theta \approx 0^{\circ}$ or $90^{\circ}$. In many images defects were observed which cause a lower number of NNs. Therefore, we also assigned $\Lambda_{0}$-defect or $\Lambda_{1}$-defect to particles for which one of the criteria was not met, see Table 7.3 for details.

\subsection{Results \& Discussion}

\subsection{Dynamics of assembly process}

The DD method was used to study the cubes dispersions during solvent evaporation. After preparation of the sample the microscope slide was directly imaged with the optical microscope. The study of a $12,5 \mu \mathrm{L}$ cube dispersion of $0.4 \mathrm{v} \%$ revealed several aspects about the assembly process. First, in the bulk suspensions single cubes that diffused freely through the solvent were observed, indicating that no aggregation or irreversible attachment to the glass surface occurs. Figure 7.4.a shows a light microscopy image of the center of the droplet where individual cubes can be clearly distinguished. Second, close to the contact line of the meniscus the cubes are pinned to the substrate. Due to their distortion of the solvent-air interface strong capillary forces caused the cubes to form close packed structures, as shown in Figure 7.4.b. The cubes in turn pin the contact line and due to the fact that the droplets still evaporates, a strong flow toward the contact line was induced. This flow transports cubes to the already formed closepacked structure causing the structure to grow over a range of several hundreds of $\mu \mathrm{m}$. Third, during the addition of cubes at the growth front, the cubes were seen to relocate and reorient into niches of the already existing structure. Rearrangements were also observed in the already formed structure further from the growth front. The final structure contained distinct ordered regions, as shown in Figure 7.4.c for the bottom layer $100 \mu \mathrm{m}$ from the droplet edge. Fourth, solvent evaporation from the structure did not seem to affect the order of the deposit. The particles were observed to shrink upon solvent evaporation and typical crack formation was seen but the order in the structures remained mostly unchanged as shown in Figure 7.4.d. It has to be noted that low concentration dispersions in which mono layers form, cubes could be seen to 'jump' out of the assemblies to another position at the moment the solvent evaporated from their core. Because only the bottom layer of the multi-layer structures could be imaged clearly, we do not know if the cubes in the top layer were not disturbed.

\subsubsection{Effect of assembly method on structure of deposit}

In the three different convective assembly methods the growth takes place in slightly different ways due to the change in shape of the contact line and meniscus. As a result, cube deposits with distinct deposit shapes are obtained for each method as shown in Figure.7.5. All obtained deposits do show strong Bragg reflections that indicates that in ordered domains of cubes have formed with all method and hence the shape of the deposit does not seem to influence the ordering process. 

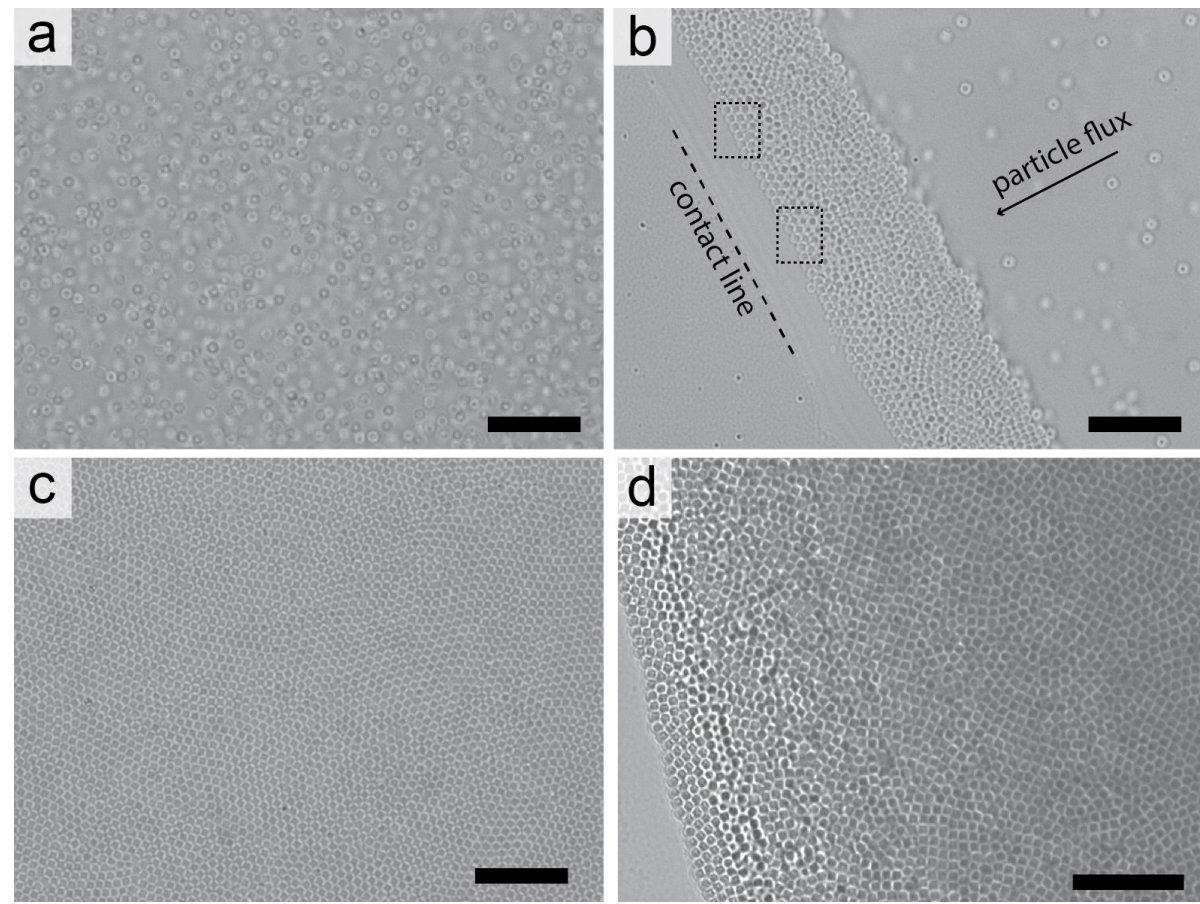

Figure 7.4. Optical microscopy images of a) bulk cube dispersion b) assembly of cubes at the contact line of the meniscus c) bottom layer of the close packed structure formed $\sim 100 \mu \mathrm{m}$ from the contact line. Distinct ordered regions with different orientations are present $d$ ) close packed structure of cubes obtained after drying. Scale bars are $10 \mu \mathrm{m}$

In the DD method evaporation occurs from all sides of the droplet and a ring-like deposit is obtained. A typical example is shown in Figure 7.5.a, with a high concentration of particles at the edges and a low concentration in the center; the arrow indicates the direction of deposit formation. In the HD method a second slide is positioned with an angle on the substrate, creating a wedge in which the dispersion droplet is placed (Fig. 7.5.b). Due to the wetting of
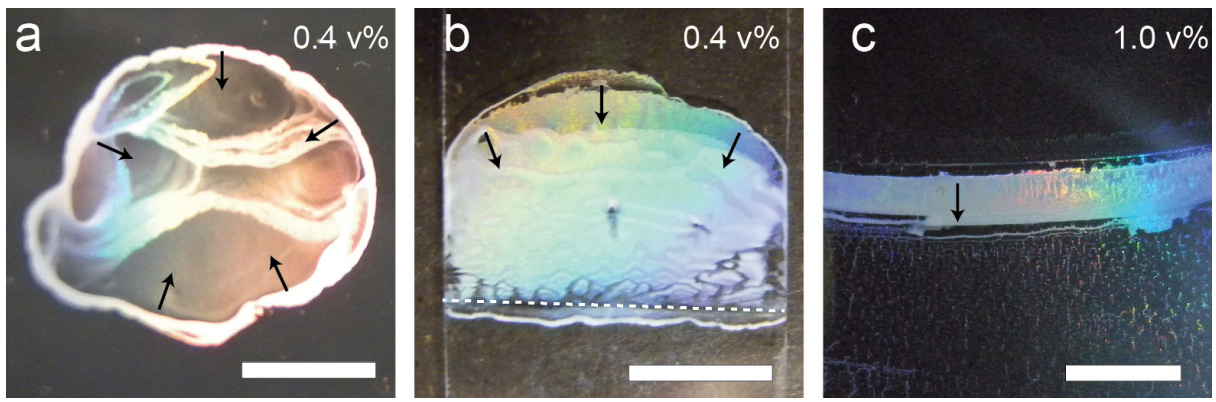

Figure 7.5. Photographs of the obtained deposits from the a) DD method and b) HD method and c) part of the deposit of the FCVD method of different cube concentrations. Scale bars are $5 \mathrm{~mm}$. 
both slides by the dispersion the meniscus shape is changed from convex to concave. Moreover, the evaporation is limited to one side of the droplet and will be fastest at the free contact line on the bottom substrate and the growth of a close-packed structure will start here. Figure 7.5.b. shows the obtained deposit, where the growth direction is indicated with the arrows and the location where the top slide was in contact with the substrate by the dashed white line. Due to the concave meniscus shape the solvent layer close the contact line is much thinner than in the DD setup. As a result evaporation will occur faster but also less particles fit in the solvent layer and a large $(\sim \mathrm{mm})$ monolayer regions is obtained at the start followed by multilayer regions toward the middle of the substrate. By changing the initial cube concentrations the length and thickness of the HD deposits can be tuned, for more detail see appendix A.7.2. In optimal conditions a deposit that covers the full bottom substrate can be obtained such as shown in Figure 7.5.b.

Note that in the DD and HD methods gravity also influences the deposition process. The cubes will sediment due to their large size, which increases the cube concentration close to the substrate. The transport of solvent in the region of the contact line will be fastest close the substrate and as the cube concentration has increase here, a larger amount of cubes will be transported to the growth front than when the dispersion would have been homogeneous.

In the FCVD method the substrate is immersed in the dispersion, which causes a concave meniscus shape and more straightened contact line than for the HD method. Figure 7.5.c shows the obtained deposition where the straightened contact line can clearly be observed. In addition, the white color of the deposit shows that the thickness is quite high which indicates that the increased dropping speed of the dispersion indeed allowed deposition of the cubes to occur in this vertical setup. The fact that the deposit is only a few $\mathrm{mm}$ in length indicates that the increase dropping speed of the meniscus did not completely counteract sedimentation and the concentration of cubes decreased repidly at the growth front after some time.

\subsubsection{Structure analysis}

The order in the close packed structures was investigated in detail with SEM. First of all we investigated the alignment of the cubes at the contact line. Figure 7.6.a shows the edge of a DD deposit where the cubes have assembled into a close-packed arrangement. Note that the cube shape of the particles is clearly visible, implying that these cubes are positioned flat on the substrate as expected (see also Chapter 6). Furthermore, cubes closest to the contact line, indicated by the dashed line, have different orientations and are not positioned on a straight line, which seems to have influenced the orientation and position of later added neighboring cubes. Figures 7.6.b-d show three higher magnifications of the cubes close to the contact line together with a schematic illustration. Clearly the orientation of the contact line does not influence the cube orientation. In Figure 7.6.b the different orientations of the cubes at the contact line can also be seen, as indicated by the dashed boxes. Further from the contact line the structure can be seen to increases stepwise to a total of four layers as indicated by the numbers. The transition regions from $n$ to $n+1$ layers do not possess the same square orientations as previously seen for 


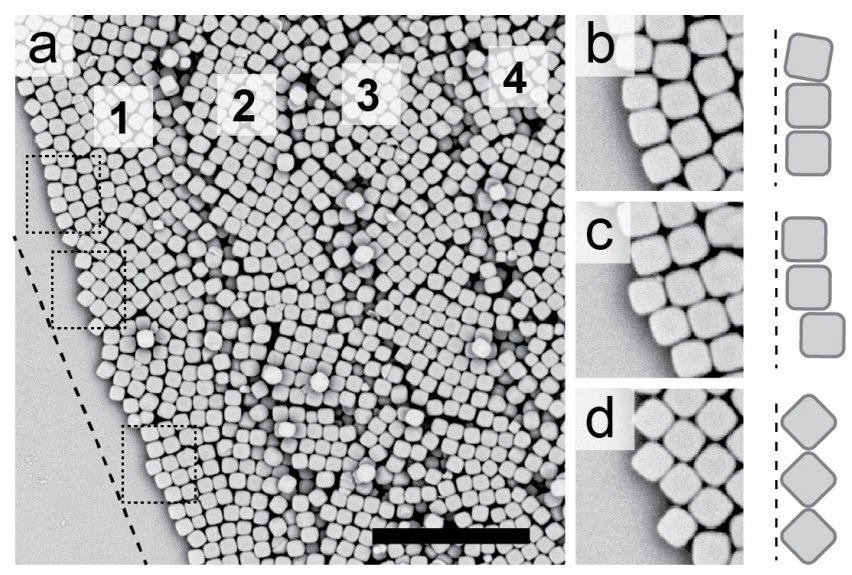

Figure 7.6. a) SEM image of the structure originally formed at the contact line (dashed line) in a DD experiment. b-d) higher magnification images as indicated by dashed squares and schematic illustrations of the different orientations and positions of the first line of pinned cubes with respect to the contact line. Scale bars in a) is $10 \mu \mathrm{m}$.
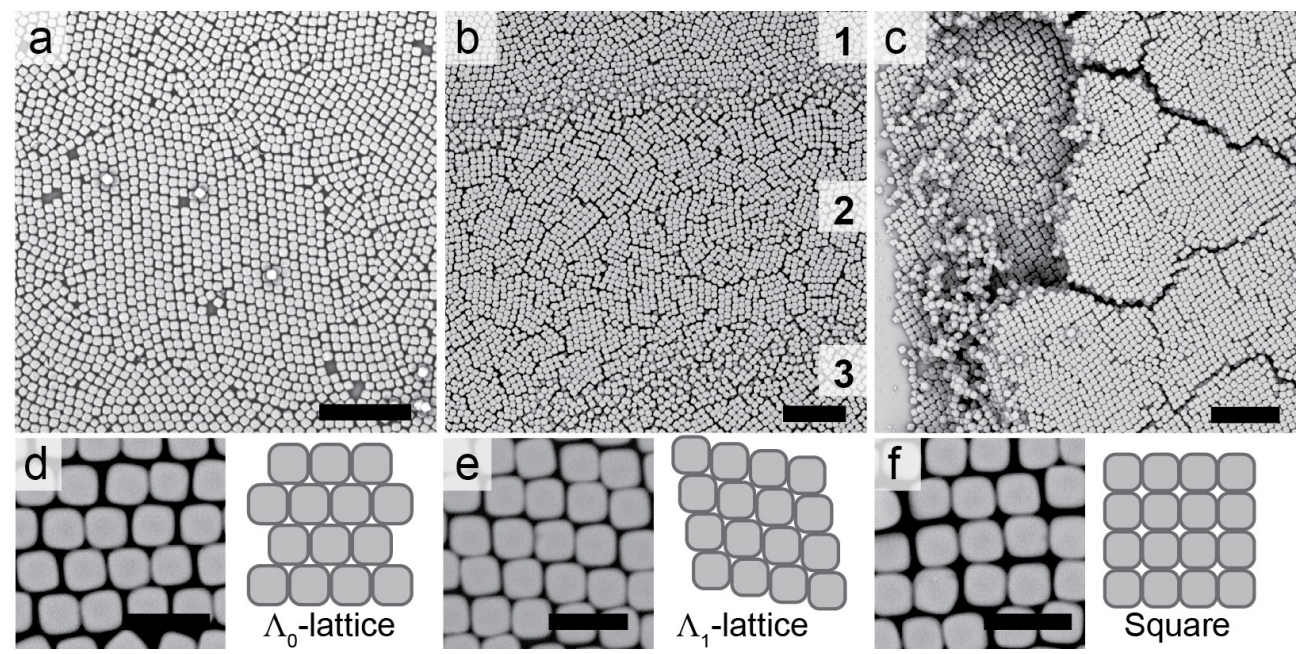

Figure 7.7. SEM Images of a HD deposit where different structures have formed. a) a monolayer b) transition of one to three layers and c) a multilayer region, by scratching the structure with a knife the inner part has been revealed showing seven layers. $d$-f) different types of packings of the cubes of d) the $\Lambda_{0}$-lattice packing e) $\Lambda_{1}$-lattice packing and f) 2D simple cubic. Scale bars are a-c) $10 \mu \mathrm{m}$ and d-f) $2 \mu \mathrm{m}$.

the layer transitions in the assemblies of spheres ${ }^{[12,16]}$. It actually seems that the orientations of the cubes in the second layer are uncorrelated to those in the first layer. Evidently, a thickness increase of the structure destroys the correlations between the cubes. Despite the difference in orientation the close-packed structures seen in each layer look similar.

As discussed above, for the concave meniscus shape in the HD method the wetting layer is 
thinner. This induces the formation of large monolayer structures as well as multilayer structures in which the stepwise addition of layers occurs over much larger distances than in the DD method Figure 7.7.a shows a typical example of such a monolayer and Figure 7.7.b. shows the increase from one to three layers over a distance of $50 \mu \mathrm{m}$. In the monolayers ordered domains with different orientations and packings can be observed. In the second layer the cubes are spaced slightly further apart, which is most likely caused by drying effects, but similar ordered regions can be observed. The order was even found to persist in the multilayer regions as shown in Figure 7.7.c for a deposit of seven layers. This multi-layer structure was scratched on the left side of the images with a knife to reveal the inner structure. The large cracks that can also be observed in the multilayered structure are well known for convective assembled structures and are caused by the high tensile stresses during drying ${ }^{[17]}$.

In the ordered structures different types of lattice packings can be identified, ranging from the theoretically densest $2 \mathrm{D} \Lambda_{0}$-lattice and $\Lambda_{1}$-lattices packings to square arrangement. (ref jiao) The densest packings were predicted for superdisks the $2 \mathrm{D}$ equivalent of superballs, are illustrated in Figure 7.7.d and 7.7.e, respectively. The square packing (Fig.7.7.f) and intermediates between the $\Lambda_{0}$-lattice and $\Lambda_{1}$-lattice packings were also observed even though these structures have a lower packing density.

\subsubsection{Lattice identification}

Closer investigations of the large monolayer suggest a tendency of the cubes to form the $\Lambda_{1}$-lattice. Figure 7.8.a-7.8.c shows three typical SEM images of monolayers in which the $\Lambda_{1}$-lattice as well as differently ordered structures and defects can be observed. In Figure 7.8.a. a continuous transformation of $\Lambda_{1}$ to $\Lambda_{0}$ seems to be present in the form of switching rows of cubes. Figure 7.8.b. shows a large region that could clearly be identified as the $\Lambda_{1}$ structure. Figure 7.8.c. shows that $\Lambda_{0}$ and square lattice were also observed but only over short distances as indicated by the hexagon and rectangles, respectively. These two lattices were usually found in the presence of defect particles and/or stacking faults, as indicated by the white arrows, that seem to be caused by the polydispersity in the particle size and shape.

To further analyze the statistics of lattice packings in cube monolayers, computer analysis routines were used for the automatic identification of the local lattice environment of a cube. First, the coordinates and orientation of each particle were determined from the SEM images by particle tracking algorithms. For the three SEM image this is shown in 7.8.d-7.8.f. The angle $(\alpha)$ the long diagonal makes with the $x$-axis is color coded with steps of $15^{\circ}$ over a range of $90^{\circ}$. This range describes all possible cube orientations because a cube has fourfold symmetry. From the correlations of the particle positions the pair correlation function $g(r)$ was determined. The $g(r)$ reveals the presence of local correlations and provided some clues of the presence of long range ordered domains in the structures, though specific assignment of a single lattice is not possible. See appendix A.7.2 for more detail.

The unique position and orientation of a cube in the $\Lambda_{0}$-lattice, $\Lambda_{1}$-lattice and square-lattice can also be used for lattice identification. Based on the positions of the nearest neighbors of the 

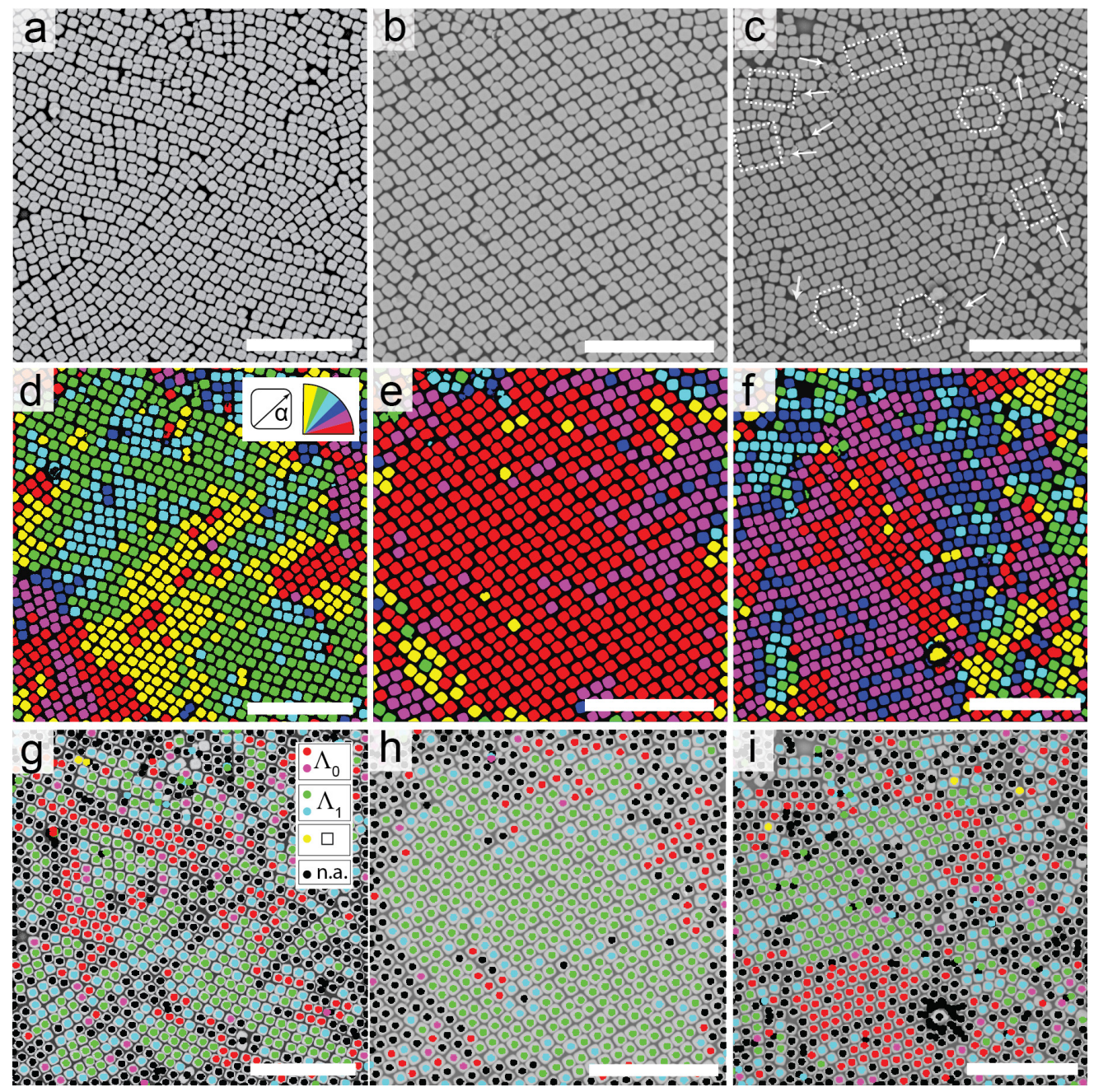

Figure 7.8. a-c) SEM images of monolayers of cubes. $d-f$ ) Result of an analysis of the particle coordinates and the orientation of the cube color coded with the orientation of the cube long diagonal. g-i) Analysis of the local particle environment, where the colors indicate (red) perfect $\Lambda_{0}$, (purple) $\operatorname{defect} \Lambda_{0}$, (green) perfect $\Lambda_{1}$, (blue) defect $\Lambda_{1}$, (yellow) square, and (black) not assigned. See Section 7.2.4 for more detail on the assignment criteria.

cubes within a cutoff radius of $1.89 \mathrm{D}$ with respect to the orientation of the cube long diagonal the local lattice were identified. Figure 7.8.g-7.8.i. show the typical result of this analysis for the three SEM images above. For the $\Lambda_{0}$ and $\Lambda_{1}$ two different lattice environments were identified. The 'perfect' lattices are those that meet all criteria, while the 'defect' lattices are assigned to those particles for which one of the criteria is not fully met. See Section 7.2.4. for more detail. These images confirm the observations made previously. In Figure 7.8.g $\Lambda_{0}$ and $\Lambda_{1}$ regions interchange over short distances. In Figure 7.8.h most cubes are identified as having local 


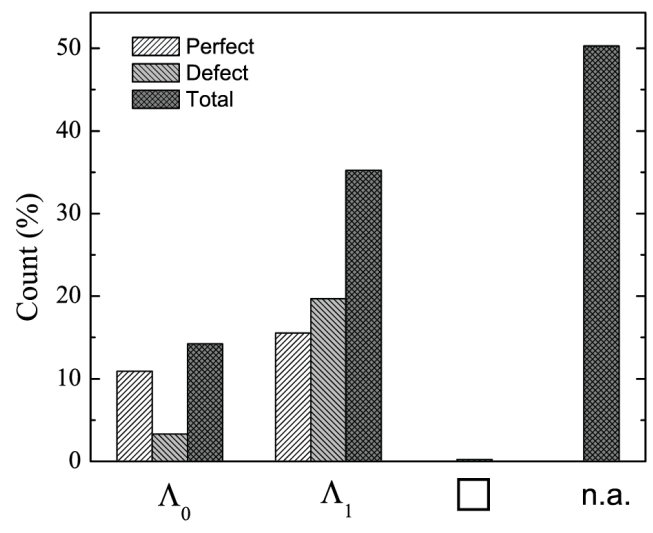

Figure 7.9. Percentage of cubes found for each lattice type, $\Lambda_{0}, \Lambda_{1}$ and square, and the cubes that were not assigned (n.a.) a lattice.

$\Lambda_{1}$-lattice arrangements. In contrast, the particles in Figure 7.8.i are mostly not assigned which is attributed to the large number of observed defects. Those particles, which could be identified, indeed show the presence of a $\Lambda_{0}$-lattice in the regions we identified with a hexagon in Figure 7.8.c.

Based on this identification procedure the presence of the lattices in the SEM images of the monolayer structures was analyzed. In total approximately 48.000 local particle environments were determined. Figure 7.9 shows the histogram with percentages of the particles found per lattice including the percentage of particles that could not be assigned a specific lattice-type. Clearly, both the 'perfect' $\Lambda_{0}$ and $\Lambda_{1}$ lattice packings are present in the layers to almost the same extend, while the square-lattice is very rare. When also taking the particles assigned a 'defect' lattice, the $\Lambda_{1}$ lattice packing is clearly the dominant structure.

That a large number of particles could not be assigned a lattice is the fact that for proper analysis of the cube orientation SEM images at high magnification have to be taken that causes many particles to be located at the image edges and cannot be assigned. In addition, cubes on top of the monolayers cause the particle tracking algorithm to identify a large number of particles around it, as can for instance be seen in Figure 7.8.i. The low number of particles with a square lattice is a consequence of the low persistence of the structure. In Figure 7.8.c it is clear that the square order extends only two rows of cubes. An improvement of both the particle tracking algorithm and analysis routine are required to reduce the unassigned number on increase the square lattice number. However, the exclusion or inclusion of these particles will probably not affect the outcome of the ratio between the $\Lambda_{0}$ and $\Lambda_{1}$ lattices.

Evidently, both $\Lambda_{0}$ and $\Lambda_{1}$-lattice packings are present in the formed monolayers, with a clear dominance of the $\Lambda_{1}$-lattice. The formation of these structures is related to the fact that they provide the optimal packings for these rounded cubes ${ }^{[3]}$. For densely packed particles systems it has been shown before that a realization of the highest possible packing densities will occur. 
For instance precious opals consisting of spheres of two different sizes were shown to form an $\mathrm{AB}_{13}$ structure $^{[18]}$ as well as more recently various binary structures were assembled form differently sized colloidal spheres and quantum dots ${ }^{[19,20]}$. Our results are however contradictory to the findings of recent experiments and simulations of rounded squares that only showed the formation of rhombic structures, which at maximum packing density are equal to the $\Lambda_{1}$ lattice ${ }^{[4,5]}$. The additional presence of $\Lambda_{0}$ here can be explained by the differences in formation mechanism. The close-packed structures obtained for the rounded squares are equilibrium structures formed by a slow increase in concentration of particles while the convective assembled structures are formed by capillary force and convective transport of the particles. In the latter strong forces are present between the particles and in addition the cubes are pushed together by the continuous addition of cubes to the growth front and the solvent flow. Therefore cubes cannot explore all their possible configurations as we also observed close to the contact line (Fig.7.6). The resulting structures will therefore contain grain boundaries and other defect structured. In addition, the curved contact line of the solvent and the polydispersity of the cubes in size and shape affect the structures.

\subsubsection{Lattice analysis of multi-layered structures}

In the top layer of multi-layered structures differently ordered lattices could also be identified and the same lattice analysis was performed. Figure 7.10.a and 7.10.b show the lattice assignment in two SEM images of the top layer from a HD deposit and a FCVD deposit. The larger distances between the particles and cracks in the structure make identification of the local order difficult, which is clear from the fact that most particles are colored black. The statistics obtained from the analysis of seven of these SEM images (18.000 particles) are shown in Figure 7.10.c. It is clear that for those particles that could be assigned a lattice, the similar $\Lambda_{0}$ and $\Lambda_{1}$ ratios are obtained as those for the monolayer. Evidently, the $\Lambda_{1}$-lattice remains the densest packing even for multilayers. However, the statistics of only seven images are of course low (over $60 \%$ has not been assigned) and only the top layer of a $3 \mathrm{D}$ structure is analyzed. Thus it is hard to draw
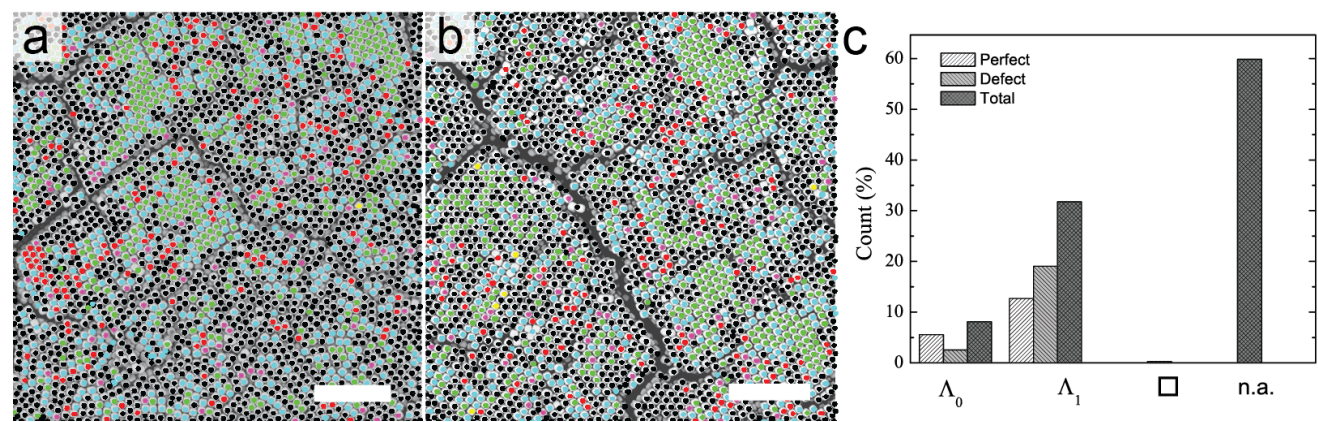

Figure 7.10 a,b) Local particle environment analysis in two multilayered structures formed by a) HD method b) FCVD method. Scale bars are $10 \mu \mathrm{m}$. c) Percentage of cubes found for each lattice type in the multi-layers and unassigned particles (n.a.). 
a conclusion about the exact order in the multi-layered structures. Information about the 3D structures could be obtained by using small angle X-ray scattering (SAXS) or with confocal laser scanning microscopy (CLSM). The SAXS results obtained for convective assembly crystals in chapter 6 and preliminary CLSM experiments have shown that this analysis route is promising and should be considered for future experiments.

\subsection{Conclusion}

The formation of close-packed crystalline structures of micrometer-sized colloidal cubes was studied in detail with three different convective assembly methods. A drying droplet method allowed direct microscopic observations of the dynamics of the structure formation. The experiments show that the cubes are pinned close the contact-line of the meniscus at different positions with a flat but otherwise random orientation. Due to the transport of cubes and the strong interparticle capillary forces this lead to the nucleation of differently oriented crystal structures. At the growth front of the crystal the cube addition occured at specific places and complete structure rearrangements were found to take place and led to the formation of longrange ordered structures over a few hundreds of micrometers.

The water evaporation and meniscus shape could be controlled by employing a horizontal deposition method using a wedge-shaped setup or with a flow-controlled vertical deposition method. These two methods allowed us to tune the thickness of the assembled structures from well-ordered monolayer and multi-layer (up to 10 layers) regions.

Analysis of the lattice packings in the monolayer structures revealed the presence of both predicted optimal $\Lambda_{0}$ and $\Lambda_{1}$-lattice packings, with a dominance of the $\Lambda_{1}$-lattice. Their formation is related to the systems realization of the highest packing densities under the convective assembly conditions. Preliminary analysis of the $3 \mathrm{D}$ structures showed that also here the $\Lambda_{1}$-lattice seems to be formed, however, more analysis is needed to determine the true $3 \mathrm{D}$ crystal structure. 


\subsection{Outlook}

The presence of different crystal structures in the assembled structures can be attributed to the curved contact line and the different orientations of the colloidal cubes already of the first ordered nuclei. A recent study has shown that the curved contact line of the meniscus is the cause of grain boundaries in monolayer of spheres. By straightening the contact line with a template containing polymer lines single lattice orientations without grain boundaries could be obtained ${ }^{[21]}$.

Preliminary experiments with the use of a template have shown that a similar effect can be achieved for these cubic particles. Figure 7.11 shows an optical microscopy and SEM image of the colloidal cubes aligned at a polymer line. Close to the polymer line the cubes are oriented with one of their faces parallel to the line and a $\Lambda_{1}$-lattice appears to have formed. It was found that the attachment of the contact line at the polymer line was not as trivial as expected and further experiments are needed to determine to which extend the structures formed by these cubes can be controlled.
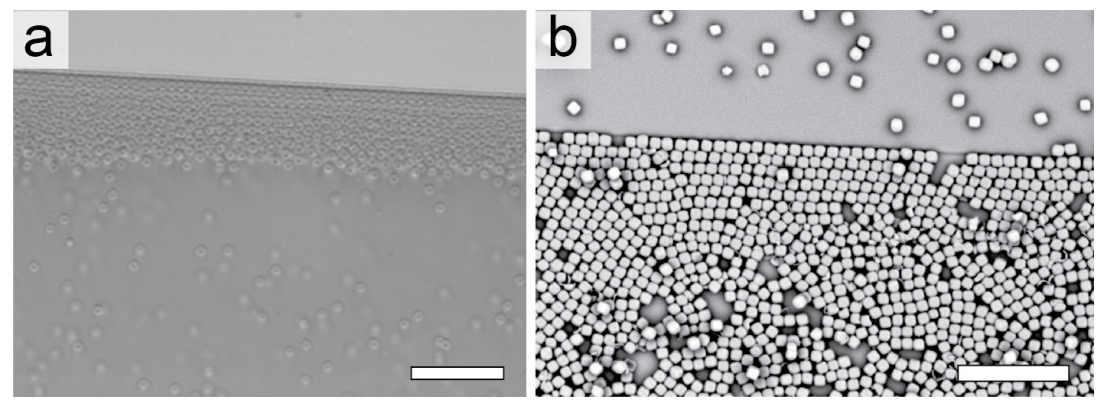

Figure 7.II. a) optical light microscopy image of the assembly of cubes at a polymer line and b) SEM images of the structure formed by the cubes at a polymer line in a DD experiment. The polymer line seems to induce the formation of a $\Lambda_{1}$-lattice. Scale bars are $10 \mu \mathrm{m}$.

\section{Acknowledgements}

This work is the result of the master thesis project of Vera Meester who performed most of the convective assembly experiments. Jan Hilhorst is thanked for help with the lattice analysis. Lisette Pompe is thanked for her contribution to the hematite cube synthesis. 


\section{Appendices}

\section{A.7.I. Determination of NNs angles}

The determination of the nearest neighbor $(\mathrm{NN})$ angles is done according to the following procedure. Figure 7.12.c. shows a schematic representation of the NNs detected for a cube orientation with $\alpha=10^{\circ}$. First, the relative positions of the NNs with respect to the center particle are determined in $\Delta x$ and $\Delta y$. Next, the angle of a NN with respect to the $x$-axis can be calculated according to $\beta=\left|\tan ^{-1}(\Delta y / \Delta x)\right|$. For those NNs with a negative $\Delta x$ or $\Delta y$ we convert this into $\gamma=90^{\circ}-\beta$, to get the same relative (dashed) angles, which can be done due to the four fold symmetry of the cubes, which is illustrated by the grey dotted lines. The angle of the NNs with the face diagonal of the cubes is then simply calculated by $\theta=\beta-\alpha$ or $\theta=\gamma-\alpha$.

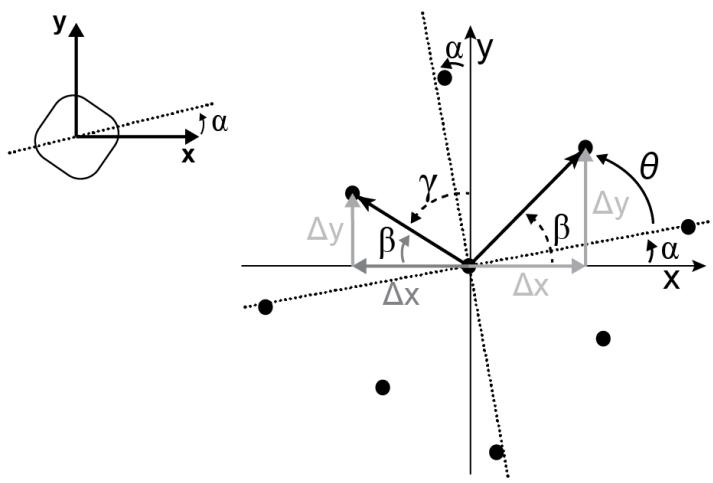

Figure 7.12. Schematic illustration of how the $N N$ angle $\theta$ is determined from the $N N$ positions.

\section{A.7.2. HD deposit shape}

The convective assembly of particles is dependent on a number of factors (see also Chapter 1 , Section 1.4). For the HD deposits it was found that the biggest effects were caused by the contact angle of the substrate and initial concentration of the cube dispersion. Figure 7.13 shows the deposits obtained for different cube dispersion concentrations. The contact angle changes with etching time of the substrate, as this is more or less hydrophilic, causing a thin or thick solvent layer at the start. The cube concentration determines the particle flux of the cubes and influences the solvent layer thickness. An increase in cube concentration results in an increase of structure thickness. Also the length of the deposit from the contact line is affected by the cube concentration. Large uncovered regions are present for the low concentration samples because after sedimentation all cubes have already been transported to the growth front. For the higher concentrations $>0.5 \mathrm{v} \%$ a uncovered region is seen as well, due to the increase in thickness of the close packed structure. This leads to a depletion of cubes in the solvent that evaporates the last. It is clear that a concentration of $0.4 \mathrm{v} \%$ cubes results in the most uniform substrate films. 


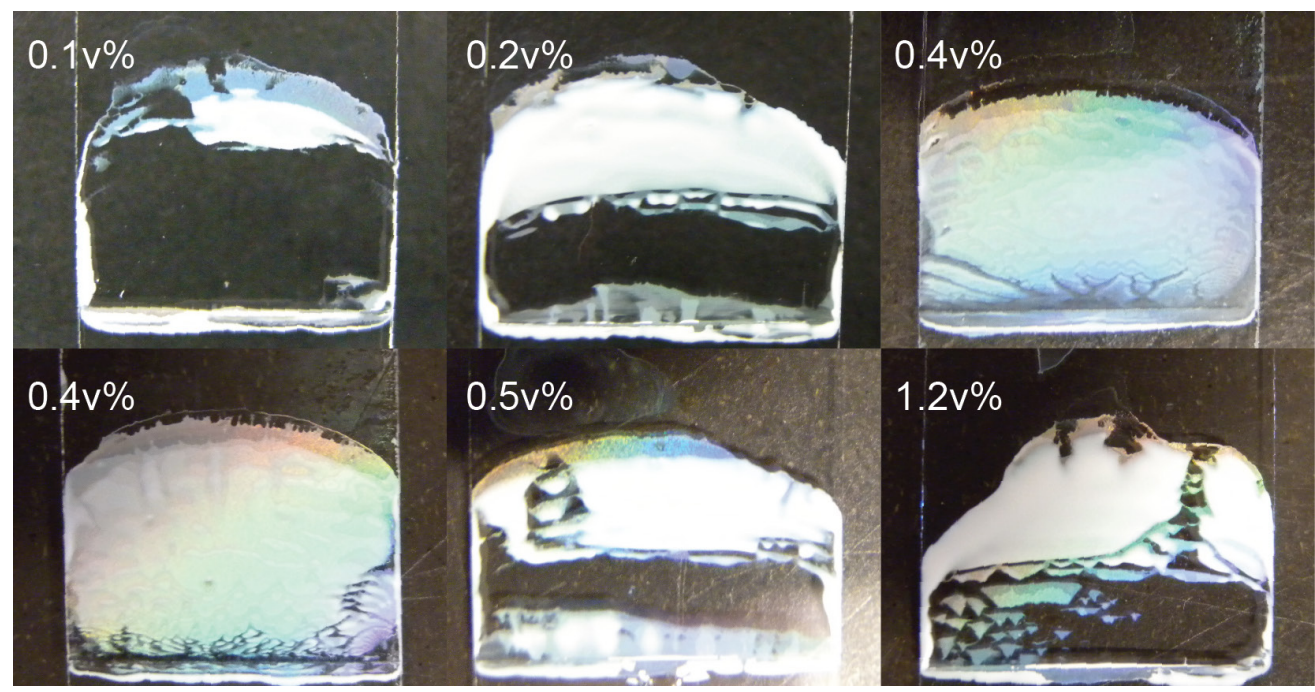

Figure 7.13. Photographs of HD deposits obtained with different dispersion concentration shown in the top left corner.

\section{A.7.3. Pair correlation function}

From the particle coordinates extracted from a SEM image the pair correlation function $g(r)$ can be determined. Figure 7.14.a shows the $g(r)$ graphs of the SEM images shown in Figure 7.8.b. and the additional images in Figure 7.14.b and 7.14.c.

All graphs show distinct peaks at distinct values of $r=1.1,2.1,3.1,4.1 \mu \mathrm{m}$ etc. up to almost 15 $\mu \mathrm{m}$. This indicates that in the SEM images long-range positional order of the cubes is present. When the peaks are compared to the expected values for the $\Lambda_{0}$ and $\Lambda_{1}$-lattice packings no correlation could be found, with of course exception of the first peak. Interestingly, the peaks in the $g(r)$ of Fig.7.8.b are slightly different from the other two and do correspond to the expected values for a $\Lambda_{1}$-lattice. However, it is clear that from these $g(r)$ curves the local structure in the monolayers cannot be extracted.
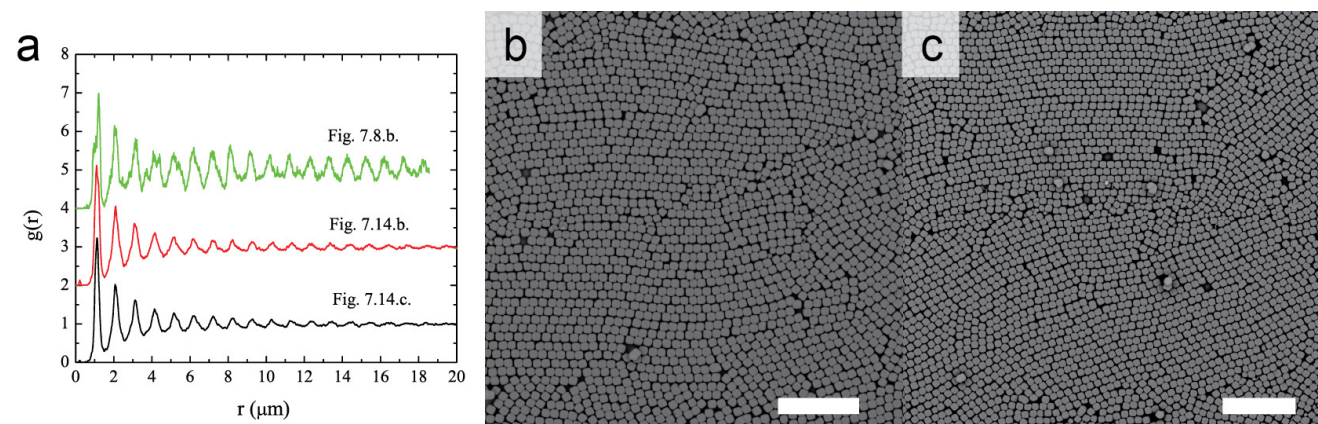

Figure 7.I4. a) $g(r)$ obtained from the different SEM image particle coordinates. b, c) SEM image of monolayers of assembled cubes. Scale bars are $10 \mu \mathrm{m}$. 


\section{References}

1. Y. Jiao, F. Stillinger and S. Torquato, Physical Review E, 2009, 79, 1-12.

2. L. Rossi, S. Sacanna, W. T. M. Irvine, P. M. Chaikin, D. J. Pine and A. P. Philipse, Soft Matter, 2011, 7, 4139-4139.

3. Y. Jiao, F. H. Stillinger and S. Torquato, Phys. Rev. Lett., 2008, 100, 245504.

4. K. Zhao, R. Bruinsma and T. G. Mason, Proc. Natl. Acad. Sci. U. S. A., 2011, 108, 2684-2687.

5. C. Avendano and F. A. Escobedo, Soft Matter, 2012, 8, 4675-4681.

6 . L. Rossi, Colloidal Superballs PhD Thesis, Utrecht University, 2012.

7. Y. Vlasov, X. Z. Bo, J. C. Sturm and D. J. Norris, Nature, 2001, 414, 289-93.

8. Z. Zhou and X. S. Zhao, Langmuir, 2004, 20, 1524-6.

9. F. Marlow, P. Sharifi, R. Brinkmann and C. Mendive, Angew. Chem., 2009, 48, 6212-33.

10. J. Zhang, Z. Sun and B. Yang, Curr. Opin. Colloid Interface Sci., 2009, 14, 103-114.

11. S. Kuai, X. Hu, a. Hache and V. Truong, J. Cryst. Growth, 2004, 267, 317-324.

12 . B. G. Prevo and O. D. Velev, Langmuir, 2004, 20, 2099-2107.

13 . B. G. Prevo, D. M. Kuncicky and O. D. Velev, Colloids Surf. A., 2007, 311, 2-10.

14 . L. B. Jerrim and O. D. Velev, Langmuir, 2009, 25, 5692-5702.

15. Z. Zhou and X. S. Zhao, Langmuir, 2005, 21, 4717-23.

16. L. Meng, H. Wei, A. Nagel, B. J. Wiley, L. E. Scriven and D. J. Norris, Nano letters, 2006, 6, 2249-53.

17 . D. J. Norris, E. G. Arlinghaus, L. Meng, R. Heiny and L. E. Scriven, Adv Mater, 2004, 16, 1393-1399.

18. J. V. Sanders, Philos. Mag. A, 1980, 42, 705-720.

19. M. E. Leunissen, C. G. Christova, A. Hynninen, C. P. Royall, A. I. Campbell, A. Imhof, M. Dijkstra, R. van Roij and A. van Blaaderen, Nature, 2005, 437, 235-240.

20 . K. Overgaag, W. Evers, B. de Nijs, R. Koole, J. Meeldijk and D. Vanmaekelbergh, J. Am. Chem. Soc., 2008, 130, 7833-7835.

21 . E. C. H. Ng, K. M. Chin and C. C. Wong, Langmuir, 2011, 27, 2244-2249. 


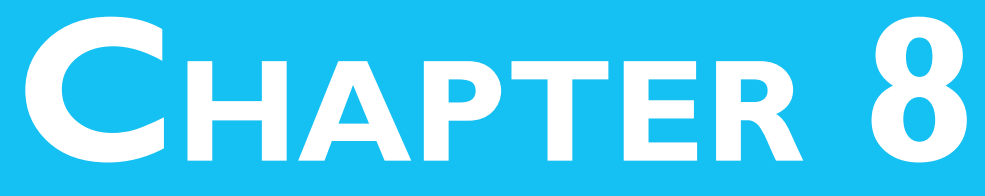

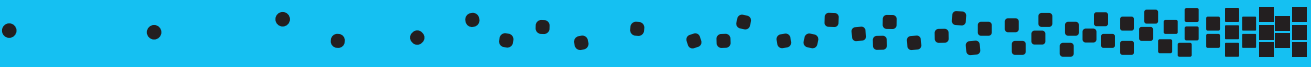 Self-Assembly of Colloidal Cubes INDUCED BY SEDIMENTATION}

Abstract

In this chapter the sedimentation of hollow silica cubes into dense packings is investigated. The effect of the Debye screening length on the close-packed structures as well as on sedimentation behavior is studied. The sediment structures are characterized in detail using small angle X-ray scattering. Remarkably, despite their rapid sedimentation the cubes order into a dense structure with both translational and orientational ordering. The structure consists of layers of cubes due to confinement between the capillary walls. The layers possess rhombic intra-layer ordering described by the $\Lambda_{1}$ lattice as predicted for $2 \mathrm{D}$ rounded cube structures and are stacked with an $A B A$ type stacking. 


\section{I. Introduction}

The phase behavior of hard cubes with rounded corners has been recently studied by computer simulations ${ }^{[1-3]}$. These simulations predict the transition from an isotropic fluid into a plastic face centered cubic (FCC) that possesses long-range translational but no orientational order, followed by the formation of a $\mathrm{C}_{1}$ crystal phases with both long-range translational and orientational order. For hollow silica cubes it has been shown that crystallization can be driven by depletion interaction ${ }^{[4,5]}$ or via capillary forces in convective assembly (Chapters 6 \& 7). However, for systems composed of particles with purely repulsive interactions, assembly into ordered phases driven only by entropic interactions is also expected ${ }^{[6,7]}$.

Therefore, in this chapter we investigate the assembly of the charged stabilized hollow colloidal silica cubes in suspension and the extent to which the rounding of the corners of the particles affects the formed structures. Moreover, we study the effect of the Debye screening length, $\mathrm{K}^{-1}$, on the self-assembly of the cubes. Due to the size of the cubes $(\sim 1 \mu \mathrm{m})$ sedimentation under gravity is unavoidable. We therefore investigate in detail the dense crystalline and disordered sediments that are obtained with small angle X-ray scattering (SAXS).

\subsection{Experimental}

\subsection{Colloidal hollow silica cubes}

Cubes were synthesized based on a hematite template cube with edge length $D=1180 \mathrm{~nm}$ according to the procedure described in Chapter 5, Section 5.2. The resulting hollow silica cubes HC4_Si_H are shown in Figure 8.1. The cubes have an edge length of $D=1266 \mathrm{~nm}$ with a polydispersity of $4 \%$. The particle shape can be modeled with that of a superball (a shape further explained in Chapter 5) with a shape parameter $m$ of 3.55 .

\subsubsection{Sample preparation}

HC4_Si_H cubes dispersions were prepared at several salt concentrations in different solvents to span a range of Debye screening length scales from $\kappa^{-1}=3$ to $100 \mathrm{~nm}$. To water from a Millipore system (resistivity $>18 \mathrm{M} \Omega . \mathrm{cm}$ ) different concentrations of $\mathrm{NaCl}$ (Merck) were added. To ethanol (p.a., Merck) different concentrations of $\mathrm{LiNO}_{3}$ (Merck, 99+\%) were added. $\mathrm{LiNO}_{3}$ was used as this salt is known to completely dissociate in ethanol ${ }^{[8]}$. The cubes were also dispersed in a $6 \mathrm{mM}$ tetramethyl ammonium hydroxide (TMAH, Fluka) solution, which has a $\mathrm{pH} \sim 9$. Cubes were transferred from ethanol to the desired solvent by repeated centrifugation and replacement of the supernatant. A waiting time of $16 \mathrm{~h}$ was always employed either after the first or second washing step to allow the hollow cubes to be completely filled with salt solution from their surroundings. The electrolyte concentrations and corresponding Debye screening length, $\kappa^{-1}$, for each sample are given in Table 8.1. Samples in water are labeled W0W4 for increasing salt concentration, in ethanol the samples are labeled E0-E3 and the TMAH sample is labeled T. Cube dispersions of typically $5 \mathrm{v} \%$ were placed in capillaries with internal dimensions of $100 \times 4 \times 0.2 \mathrm{~mm}$ (W3520 Vitrocom) that were flame sealed and stored vertically. Sedimentation occurred over a period of 24-72 hours. 


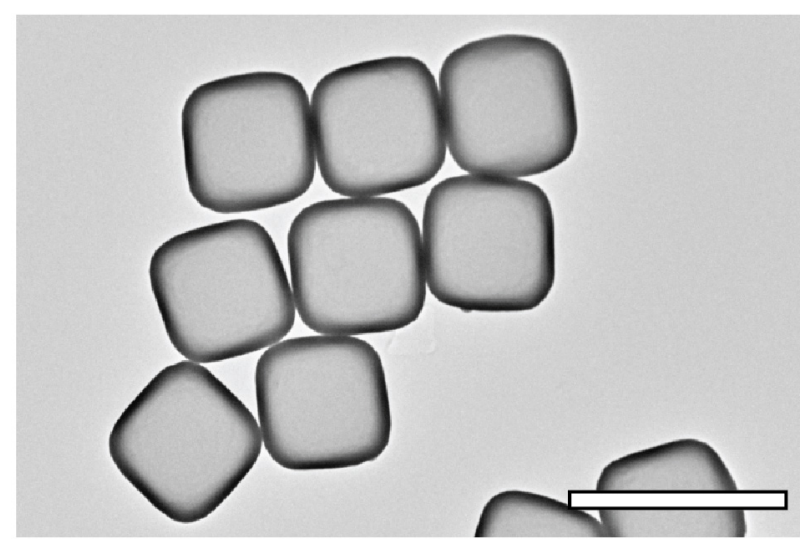

Figure 8.I. TEM image of the colloidal hollow silica cubes HC4_Si_H. Scale bar is $2 \mu \mathrm{m}$.

Table 8.I. Electrolyte concentrations and Debye lengths of the different samples prepared of HC4_Si_H.

\begin{tabular}{l|ccrc}
\hline Sample & Solvent & Salt & $\begin{array}{r}\text { Concentration } \\
(\boldsymbol{\mu M})\end{array}$ & $\begin{array}{c}\boldsymbol{\kappa}^{-1} \\
(\boldsymbol{n m})\end{array}$ \\
\hline W0 & $\mathrm{H}_{2} \mathrm{O}$ & - & - & $>100$ \\
W1 & $\mathrm{H}_{2} \mathrm{O}$ & $\mathrm{NaCl}$ & 10 & 97 \\
W2 & $\mathrm{H}_{2} \mathrm{O}$ & $\mathrm{NaCl}$ & 42 & 48 \\
W3 & $\mathrm{H}_{2} \mathrm{O}$ & $\mathrm{NaCl}$ & 1020 & 10 \\
W4 & $\mathrm{H}_{2} \mathrm{O}$ & $\mathrm{NaCl}$ & 10200 & 3 \\
\hline T & $\mathrm{H}_{2} \mathrm{O}$ & $\mathrm{TMAH}^{2}$ & 6000 & 4 \\
\hline E0 & $\mathrm{EtOH}$ & - & - & 75 \\
E1 & $\mathrm{EtOH}$ & $\mathrm{LiNO}_{3}$ & 20 & 34 \\
E2 & $\mathrm{EtOH}$ & $\mathrm{LiNO}_{3}$ & 270 & 9 \\
E3 & $\mathrm{EtOH}$ & $\mathrm{LiNO}_{3}$ & 2040 & 3 \\
\hline
\end{tabular}

\subsubsection{Small angle $x$-ray scattering}

Small angle X-ray scattering (SAXS) measurements were performed at beam-line BM26B DUBBLE ${ }^{[9-11]}$ at the ESRF in Grenoble using a $\mu$ rad-XRD setup employing compound refractive lenses (CRL) $)^{[12-14]}$. The X-ray beam with an energy of $13 \mathrm{keV}$ was focused $7.17 \mathrm{~m}$ behind the sample on a CCD X-ray detector with a pixel size of $9 \times 9 \mu \mathrm{m}$ (Photonic Science). The detector was protected from the direct X-ray beam using a wedge-shaped beam-stop. The X-ray beam size at the sample was approximately $500 \times 500 \mu \mathrm{m}$. The capillaries were oriented with their short axis $(0.2 \mathrm{~mm})$ parallel to the $\mathrm{X}$-ray beam and the long axis $(100 \mathrm{~mm})$ parallel to the gravitational field. Measurements were performed at several heights in the sediment as well as rotation scans around the sample vertical axis over a range of $\omega \pm 70^{\circ}$, with $\omega=0^{\circ}$ the starting orientation. 


\subsection{Results and Discussion}

\subsection{Effect of ionic strength on crystallization}

Figure 8.2 shows the obtained hollow silica cube sediments in water at different ionic strengths under white light illumination. The differences in height between the sediments in $\mathrm{W} 0, \mathrm{~W} 1$ and $\mathrm{W} 2$ are caused by small deviations in starting concentration. Bragg reflections in these three samples indicate that at electrolyte concentration up to $42 \mu \mathrm{M}$ crystals have formed at the top of the sediments (Fig. 8.2.a-c). The part of the sample that shows Bragg reflections increases with increasing salt content; an indication that a shorter Debye length allows the cubes to rearrange to an ordered, close-packed sediment. The bottom parts of the sediments are not ordered which is probably caused by fast compression of this part of the sediment as it formed within 12 hours. Upon increasing the ionic strength above $1020 \mu \mathrm{M}$ in the W3 and W4 sample Bragg reflections are absent (Fig.8.2.d-e). Moreover, the sediments increase in height even though all samples had similar starting concentrations of $\sim 5 \mathrm{v} \%$. Evidently, aggregation occurred at these salt concentrations even though the Debye length is $\kappa^{-1}=10 \mathrm{~nm}$. The fact that crystallization only occurred in such a small salt window is puzzling as usually silica colloids are stable at higher salt concentration ${ }^{[8]}$.

The hollow silica cubes sediments in ethanol showed no visible signs of crystallization, except a small region at the top of the E0 sediment (not shown). The absence of crystals from most samples indicates that in ethanol the double-layer repulsion between the cubes is not sufficient to allow ordering in the close packed sediments. Figure 8.2.f shows the E3 sample sediment with the highest salt concentration of $2040 \mu \mathrm{M}$. In this sample a large fractured sediment with regions depleted of particles has formed indicating the aggregates are quite rigid, implying that particles are clustered due to strong van der Waals forces.

In order to understand the absence of crystals at salt concentrations above $1 \mathrm{mM}$, the sedimentation process was investigated. Figure 8.3.a shows the meniscus of the W0, W2 and W4 samples at three different times after homogenization. Surprisingly, gelation is observed in the W2 and W4 samples. This is most clear after $59 \mathrm{~min}$ as fractures have formed near the gel-solvent interface due to the gel collapsing under its own weight. Over time the gel structure relaxes and a flat dispersion-solvent interface is obtained, although for the W4 sample the interface still seems to contains aggregates. The interface slowly moves downward and Figure 8.3.b shows the distance from the meniscus to the dispersion-solvent interface over time. Clearly, the sedimentation speed of the suspensions increases with increasing salt concentration. This increased sedimentation speed together with the formation of a gel indicate that attractive forces are present between the cubes, already at the low salt concentration of $42 \mu \mathrm{M}$.

The fact that crystals are only observed in the W0, W1, W2 samples and only to a small extend in the E0 sample (not shown) indicates that crystallization occurs above a critical Debye screening length. For a shorter Debye length the cubes apparently become sticky due to van der Waals attraction, which leads to the formation of disordered structures, as seen for W3, W4 and E3. However, at $\kappa^{-1}=48 \mathrm{~nm}$ (W2) both gelation and crystallization are observed. We have no clear scenario yet for this time sequence of gelation and crystallization in one and the same 


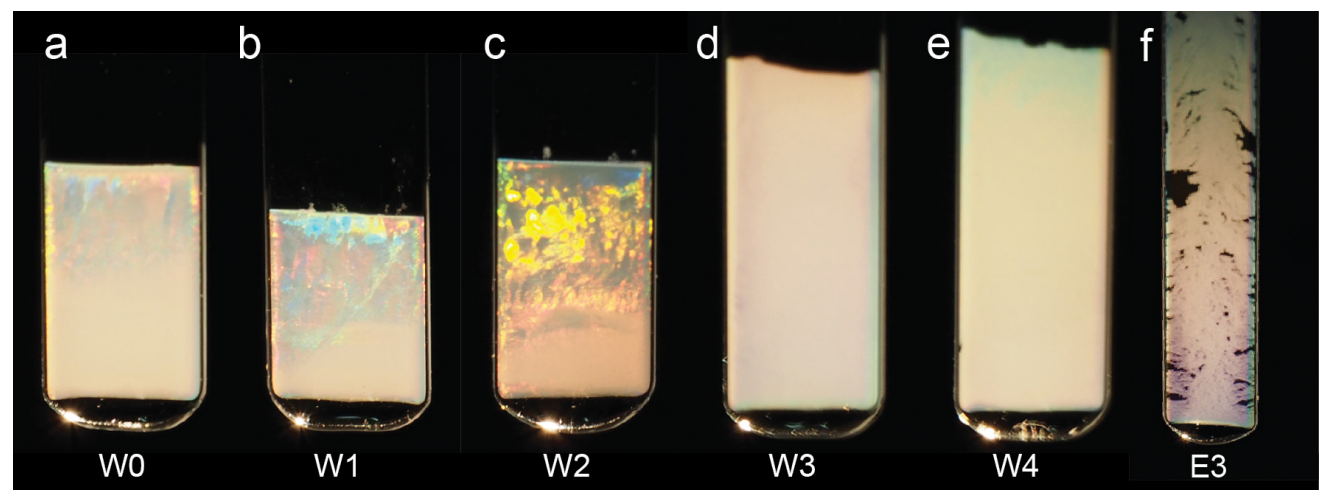

Figure 8.2. (a-e) Photographs of sediments of the different aqueous HC4_Si_H cube dispersions (W series) with different $\mathrm{NaCl}$ concentrations under white light illumination. The Debye screening length decreases per sample with $\kappa^{-1}=$ a) > $100 \mathrm{~nm}$, b) $97 \mathrm{~nm}$ c) $48 \mathrm{~nm}$, d) $10 \mathrm{~nm}$ and e) $3 \mathrm{~nm}$. f) sediment obtained from a hollow silica dispersion in ethanol of sample E3 with $2040 \mu \mathrm{MLiNO}_{3}$ resulting in $\mathrm{K}^{-1}=3 \mathrm{~nm}$.
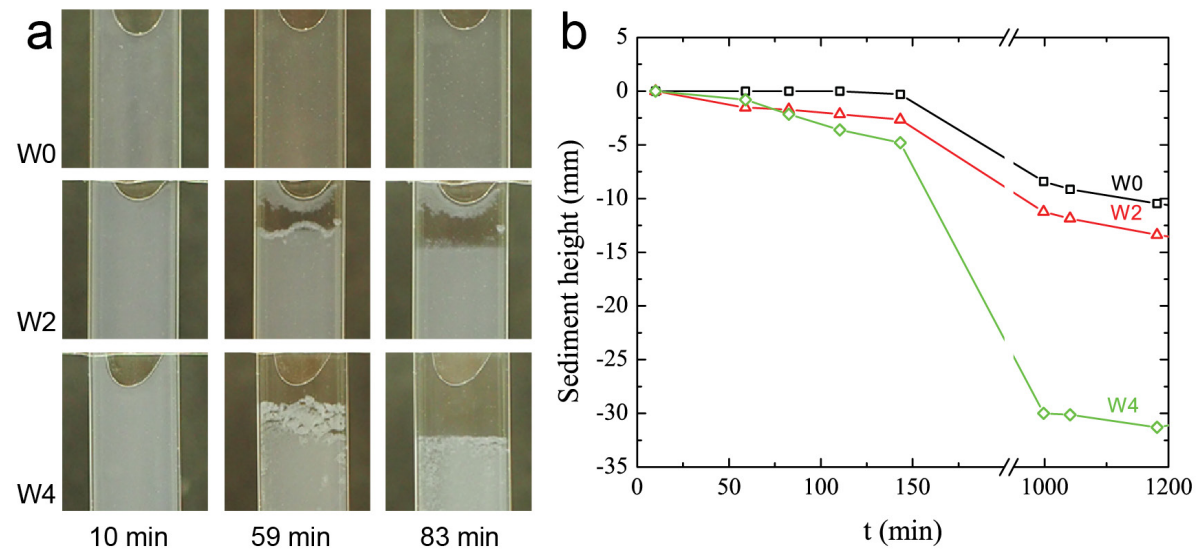

Figure 8.3. a) Dispersion-solvent interface at different times after homogenization for aqueous samples W0, W2 and W4. b) Sediment height followed over time.

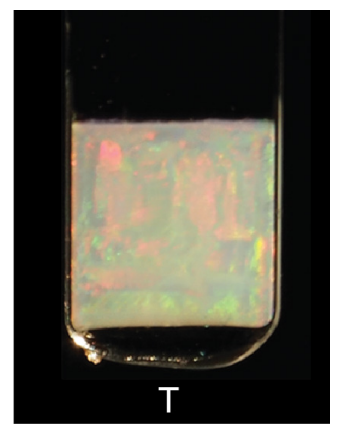

Figure 8.4. Sample T sediment containing $6 \mathrm{mM}$ TMAH illuminated with white light. 


\section{W2 sample.}

The amorphous hollow silica cubes are negatively charged due to the dissociation of silanol groups on the surface. Silanol is a weak acid and in water only a low number density of groups on a cube surface will be dissociated. A way to increase the surface charge is by elevating the $\mathrm{pH}$ of the solvent as this increases the dissociation of the silanol groups. However, this $\mathrm{pH}$ increase will finally cause etching of the silica surface. Therefore, we increased the $\mathrm{pH}$ of the cube dispersions with TMAH which is also a surface active molecule that might protect the silica surface. Figure 8.4 shows the obtained cube sediment in a $6 \mathrm{mM}$ TMAH solution. Distinct Bragg reflections are present indicating that the sediment is now fully crystalized even though $\kappa^{-1}=4 \mathrm{~nm}$. Clearly, the double layer repulsion between the cubes now suffices to outweigh the van der Waals attractions.

\subsubsection{Crystal structure analysis}

Small angle X-ray scattering (SAXS) measurements with microradian resolution were performed to determine the exact structure of the hollow silica cube sediments. Height scans along the sediments reveal the presence of order as well as disorder in the samples. The scattering data from unordered sample/regions are not shown here. Their general features closely resembled that of the form factor $P(q)$.

For the W0, W1, W2 and T samples the 2D SAXS patterns reveal the presence of distinct Bragg peaks at heights corresponding to the regions in which Bragg reflections were observed in the previous section. In addition, single crystal as well as polycrystalline domains are observed in the crystalline parts of the samples. Figure 8.5 shows the 2D SAXS patterns obtained at single crystal domain positions of the $\mathrm{W} 0, \mathrm{~W} 1, \mathrm{~W} 2$ and $\mathrm{T}$ samples which serve as representative patterns for the crystal structure in each sample. The white wedge in the patterns is the shadow of the beam stop that protects the detector from the direct beam.

The patterns reveal several aspects about the structures formed by the cubes. First of all in the 2D SAXS pattern of sample W0 in demi-water shows only low order Bragg peaks while in the SAXS pattern of the $\mathrm{W} 1$ sample at $10 \mu \mathrm{M} \mathrm{NaCl}$ higher order Bragg peaks have appeared (Fig.8.5.a,b). For the W2 sample at $42 \mu \mathrm{M} \mathrm{NaCl}$ only the $2^{\text {nd }}$ order peaks are visible in the pattern (Fig.8.5.c). This means that the increase in salt concentration from W0 to W1 increases the long-range order in the sediment, while a further increase from W1 to W2 decreases the ordering. Secondly, the patterns of the T sample with $6 \mathrm{mM}$ TMAH shows distinct Bragg peaks up to the fifth order (Fig.8.5.d). Indicating that the increase in surface charge with the simultaneous decrease of the Debye length to $\kappa^{-1}=4 \mathrm{~nm}$ by the addition of TMAH results in a long-range ordered single crystal structure. Thirdly, the SAXS patterns of T and also W1 show distinct features at high $q$ with fourfold symmetry that are most likely a consequence of a cubic form factor. This means that the cubes ordered on the crystal lattice all have similar orientations. The high $q$ features are (almost) absent for the W0 and W2 samples and here the cubes possess more random orientations. Finally, the crystal structures that have formed in all four samples have a similar rhombic character and appear to be a consequence of the particle shape. Figure 


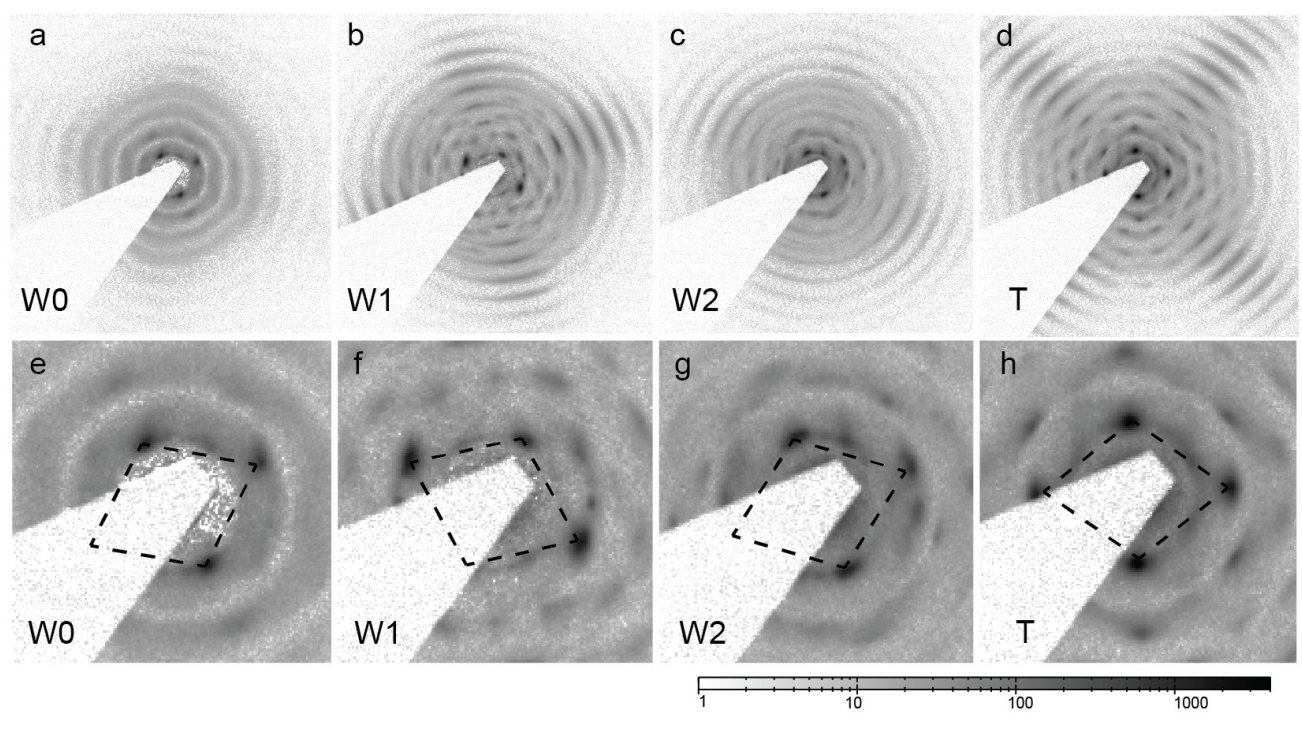

Figure 8.5. 2D SAXS patterns of a) WO b) WI c) W2 and d) T samples obtained of single crystal regions. e-h) higher magnifications of the low order Bragg peaks in each sample.

8.5.e-h shows magnifications of the low order peaks where the rhombic symmetry is indicated by the dashed lines.

Because all the samples possess similar rhombic phases we focus on the sample T pattern for the structure analysis. The $q$ dependent intensity profiles $I(q)$ along the different Bragg peaks were extracted from the scattering pattern along the lines in Figure 8.6.a and are shown in Figure 8.6.b. The normalized azimuthal $\varphi$ dependent intensity profiles shown in Figure 8.6.c. were extracted along the dashed circles in Figure 8.6.a. In the case of the high $q$ peaks the region between the two circles was integrated. From the plots the peak positions were determined by Lorentzian fitting.

The two Bragg peaks with rhombic symmetry are located at (black line) $q_{1}=5.903 \mu \mathrm{m}^{-1}$ and (blue line) $q_{2}=8.057 \mu \mathrm{m}^{-1}$ and their scattering vectors are rotated $90^{\circ}$ with respect to each other. Of these two peaks also the $2^{\text {nd }}$ order can be also observed in the profile plots. The $q_{1}$ and $q_{2}$ values correspond to real-space periodicities of $d_{1}=1.065 \mu \mathrm{m}$ and $d_{2}=0.780 \mu \mathrm{m}$ oriented perpendicular to each other. Two higher order peaks (green lines) are located at $q_{3}=10.166$ $\mu \mathrm{m}^{-1}$ and their scattering vector are rotated $70^{\circ}$ with respect to each other, $55^{\circ}$ with that of $q_{1}$ and $35^{\circ}$ with that of $q_{2}$. These $q_{3}$ peaks corresponds to real-space periodicities of $d_{3}=0.618 \mu \mathrm{m}$ and indicate that this might be a $2^{\text {nd }}$ order peak as it is close to $D / 2$. The high $q$ features (red line) showed peaks at $q_{h}=18.993,23.911,28.796,33.668$ and $38.582 \mu \mathrm{m}^{-1}$. These peaks have a periodic spacing of $\langle\Delta q\rangle=4.897 \mu \mathrm{m}^{-1}$ corresponding to a real space value of $\Delta d_{h}=1.283 \mu \mathrm{m}$. It has to be noted that the high intensities of these features compared to the other profiles plots cannot originate from the form factor $P(q)$ alone and must have a $S(q)$ origin as well, which is interesting as $\Delta d_{h}$ is very close to the particle edge length $D=1.266 \mu \mathrm{m}$. 

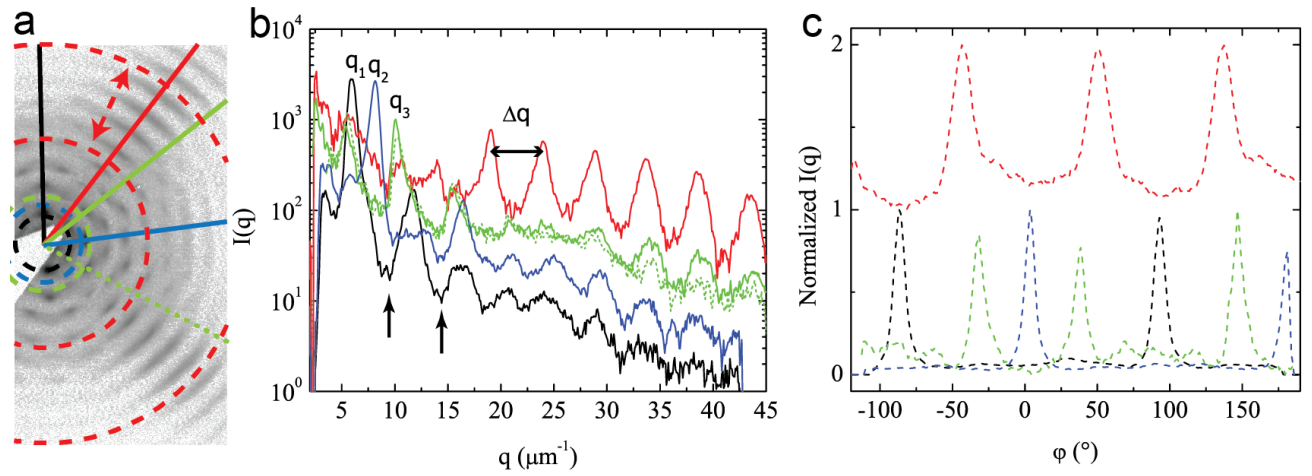

Figure 8.6. a) 2D SAXS pattern obtained in the single crystal domain of sample $T$. b) The $q$ dependent intensity profiles of the Bragg peaks along the lines in a). The plots are offset vertically for clarity. c) Normalized $\varphi$ dependent intensity profiles along the dashed circles in a).The plots of the features at high $q$ is offset by I for clarity.

In the profile plots also differences in the $\mathrm{P}(q)$ minima are observed between the $q_{3} \& q_{h}$ and the $q_{1} \& q_{2}$ peaks. The arrows in Figure 8.6.b indicate the positions where a minimum is present in $q_{1}$ and $q_{2}$ while a maximum can be observed in $q_{3}$ and $q_{h}$. Based on a the analysis described in appendix A.6 of Chapter 6, the $P(q)$ of hollow silica cubes can be approximated by that of a spherical shell. The best fits were found for shell dimensions that correspond to the edge lengths $D$ for $q_{3, h}$ and face diagonals $L$ (determined by Eq. (5.2)) for $q_{1,2}$ of the hollow silica cube interior and exterior. This means that the cubes are oriented with one face against the capillary wall with their faces oriented with a $45^{\circ}$ rotation with respect to the vertical direction. The alignment of a face parallel to the glass wall is not surprising, as colloidal rods and plates show similar anchoring behavior at glass walls. Furthermore, the sediment bulk will exert some pressure on the cubes close to the glass wall.

A schematic representation of the sedimentation process and the induced cube orientation at the capillary wall is shown in Figure 8.7. By the exerted pressure of the bulk of the sediment the orientation of the cubes a few cube positions away will also be aligned to the glass wall and as a result layers of cubes will form. In these layers ordering due to the rounded square shape will most likely occur. The capillary is $200 \mu \mathrm{m}$ thick and with $D=1.266 \mu \mathrm{m}$ this means that only 158 cubes can be arranged side to side within this direction and thus the wall anchoring will have a strong effect on the overall structure. To test the effect of the walls a cube dispersion in TMAH was allowed to settle into a round capillary of $1 \mathrm{~mm}$ internal diameter. Figure 8.7.d shows the typical scattering pattern obtained in the sediment of this round capillary sample. It is clear that a low $q$ several Bragg peaks are visible and at high $q$ ring like features can be observed. All these observations indicate that a round capillary indeed induces the formation of a polycrystalline structure.

Now that the cube orientation and the periodicities with respective angles of the crystal lattice in the layers have been established in the T sample crystal structure, we can compare our results 

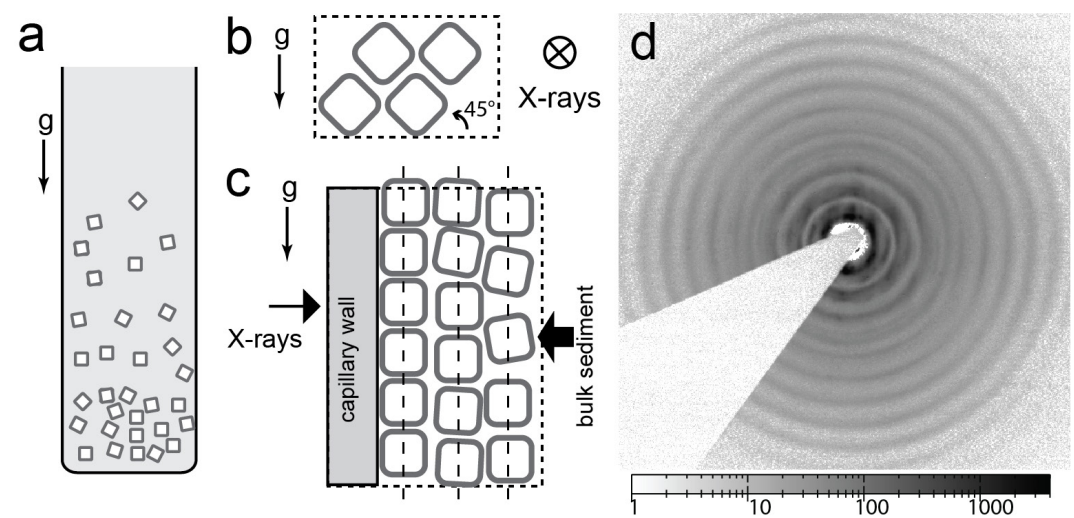

Figure 8.7. Schematic representation of a) the sedimentation process in the capillary. b) Front view of the obtained cube orientation against the glass wall as observed along the x-ray beam. c) Side view the capillary where alignment of the cubes with the capillary wall causes layers of cubes to form. d) 2D Scattering pattern obtained from sample $\mathrm{T}$ in a $\mathrm{I} \mathrm{mm}$ round capillary.

to the predicted structures for superballs. Recently, experimental ${ }^{[15,16]}$ and simulations ${ }^{[1-3,17,18]}$ studies of superball-like particles have shown the formation of rhombic crystal phases, see also Chapter 1, Section 1.3. The rhombic crystal lattices are described by the $\Lambda_{1}$-lattice in $2 \mathrm{D}^{[17]}$ and the $\mathrm{C}_{1}$-lattice in $3 \mathrm{D}^{[1]}$ with the exact lattice depending on the superball shape parameter $m$. In both structures it is the rounded corners of the cubes that are touching and cause a deformation from an hexagonal in 2D or face centered cubic (FCC) lattice in 3D that would form for spheres with $m=2$. Because the cubes have ordered into layers the $\mathrm{C}_{1}$-lattice can be excluded as it does not possess such layers. The $\Lambda_{1}$-lattice on the other hand described the order obtained within planes of superdisks (the 2D analogue of superballs) which is similar to the order in layers of superballs here.

In the T sample with $6 \mathrm{mM}$ TMAH the Debye length is $\kappa^{-1}=4 \mathrm{~nm}$ such that the double layer repulsion is of very short range in comparison to the cube edge length $D=1266 \mathrm{~nm}$. We therefore calculated the $\Lambda_{1}$-lattice vectors $\mathbf{e}_{1}$ and $\mathbf{e}_{2}{ }^{[17]}$ for a superball with $D=1266 \mathrm{~nm}$ and $m$ $=3.55$. From the $\mathbf{e}_{1}$ and $\mathbf{e}_{2}$ vectors the real-space basis lattice vectors $\mathbf{a}_{1}=\mathbf{e}_{2}$ and $\mathbf{a}_{2}=\mathbf{e}_{1}-\mathbf{e}_{2}$ are constructed. These basis vectors are chosen such that for $m=2$ these correspond to those used to describe a typical hexagonal lattice. The crystal structure is of course $3 \mathrm{D}$ and we take $\mathbf{a}_{3}$ to be perpendicular to the plane described by $\mathbf{a}_{1}$ and $\mathbf{a}_{2}$. Figure 8.8.a shows a schematic representation of the real-space vectors. From the real space vectors the reciprocal basis vectors $\mathbf{b}_{1}, \mathbf{b}_{2}$ and $\mathbf{b}_{3}$ can be determined in the usual way ${ }^{[19]}$. The vectors $\mathbf{b}_{1}$ and $\mathbf{b}_{2}$ describe the scattering originating from the layers of cubes. The values of the different $q_{h k l}=h \mathbf{b}_{1}+k \mathbf{b}_{2}+l \mathbf{b}_{3}$ for integer values of $h$ and $k$ and $l=0$ were compared to the experimental Bragg peak $q$-values. It was found that these describe the observed SAXS pattern very well.

Figure 8.8.b shows the assignment of all the Bragg peaks according to their $h k l$ indices. The assignment shows that the $q_{1}$ and $q_{2}$ peaks are the -110 and 110 peaks and not the first order 

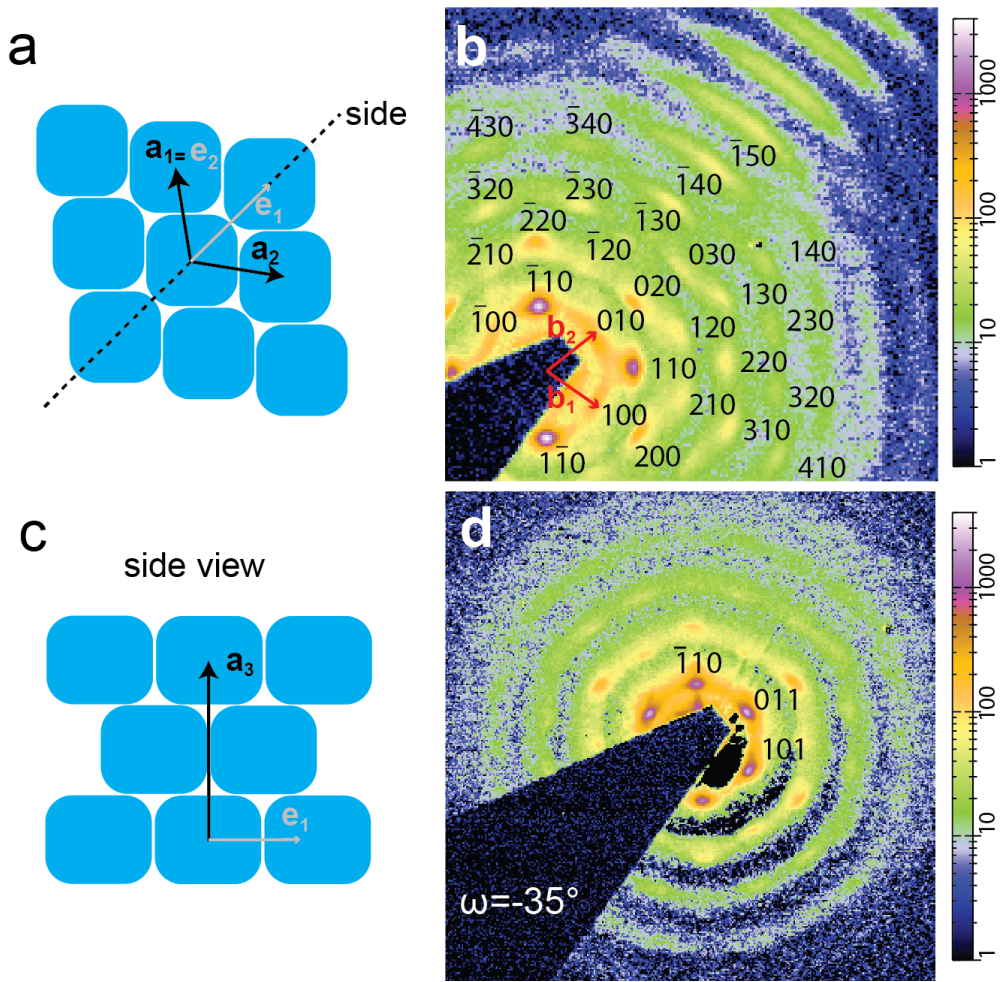

Figure 8.8. a) Schematic representation of the real-space vectors of the $\Lambda_{1}$-lattice. b) Part of diffraction pattern of the single crystal domain in sample T. The reflections are characterized with their hkl indices. c) Side view of the crystal structure along the dashed line in a). d) Diffraction pattern after a sample rotation around the vertical axis by $\omega=-35^{\circ}$.

peaks as assumed before. The 100 and 010 peaks are absent from the pattern due the fact that these overlap with a minima in the form factor of the cube face. Based on the $\Lambda_{1}$-lattice vectors the -110 reflection can be used to determine to the inter-particle spacing that corresponds to the cube edge length within the crystal. For this crystal $D_{\text {crystal }}=1.279 \mu \mathrm{m}$ which corresponds very well to $D=1.266 \mu \mathrm{m}$. The peak assignment showed that the high $q$ features overlap with higher order Bragg peaks but the fact that these are smeared show that these do not arise due to true long-range order of the crystal in this direction. They are, however, a consequence of the cube flat faces. Over long distances the rows of cubes might shift slightly in position destroying the long range order of the $\Lambda_{1}$-lattice, however due to the cube flat faces the rows are still spaced $\sim D$ apart. This periodicity explains the high $q$ features which are also amplified by the overlap with the cube form factor intensity $P(q)$. See Appendix A.8. for more details.

The $3 \mathrm{D}$ structure of the cube crystal was revealed by rotating the sample around its vertical axis. Over a full range of $\omega$ the vertical -110 peak remained visible. At rotations in the range of $\pm 40^{\circ}<\omega< \pm 20^{\circ}$ additional peaks appeared. Figure 8.8.d shows the pattern at $\omega=-35^{\circ}$ with hexagonal symmetry. The strongest inner peaks have their maximum intensity at $\omega=-35^{\circ}$ and 
are located at $q=5.61 \mu \mathrm{m}^{-1}$. These peaks were identified as the 011 and 101 peaks of a structure consisting of layers with $\Lambda_{1}$-lattice stacked in an ABA fashion. Figure 8.8.c shows a schematic the side view of this stacking sequence. In this structure a cube in second layer is positioned on the gap formed by four cubes in the first layer. The shift in layer position corresponds to $1 / 2 \mathbf{e}_{1}$. The real-space unit cell containing two cubes is described by $\mathbf{a}_{1}, \mathbf{a}_{2}$ and $\mathbf{a}_{3}$ of which the latter points upwards to a cube in the third layer according to $\mathbf{a}_{3}=2 \mathrm{Dk}$. An ABA stacking sequence results in stacking dependent and stacking independent reflections which in addition to the $P(q)$ minima explain the absence of certain peaks, such as the 001 and 010 , in the SAXS patterns at $\omega=0^{\circ}$.

\subsubsection{Crystal structure at sediment top}

Besides single crystal structures in the center of the crystalline sediments interesting features were observed in the patterns at the top of the sediments. Here the osmotic pressure is lower than in the parts of the sediment where the single crystal structures were observed. Figure 8.9.a-c show the SAXS patterns obtained at the top of the sediments of the W1, W2 and T samples. Interestingly, again similar patterns are observed for the three samples and this time the peaks are present up to similar higher orders indicating the same structures have formed at the sediment top for these three samples. At low $q$ several Brag reflections are visible and also at high $q$ features appear. These high $q$ features indicate that the cubes are oriented with their face parallel with respect to the vertical direction. Upon closer inspection the Bragg peaks again show rhombic ordering but now two distinct crystal orientations appear to be present, as indicated by the white and black dashed lines in Figure 8.9.d.

Analysis of the peak $q$ and azimuthal positions shows that these two reciprocal space lattices are rotated by $70^{\circ}$ as shown in Figure 8.10.a. In both lattices one of the b vectors is pointing vertically and as a result the reciprocal lattice points of the two orientations fall on vertical lines. In the real-space this corresponds to the lattice orientation as shown in Figure 8.10.b. In these structures the cubes have reoriented such that they are now in contact with another nearest

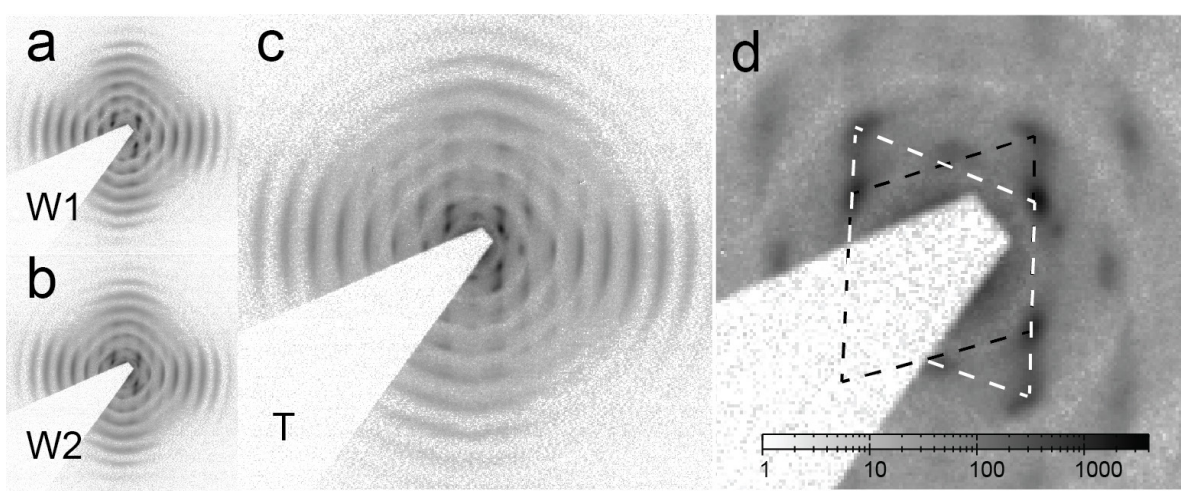

Figure 8.9. a-c) SAXS pattern at top of sediments of the WI, W2 and T samples. d) magnification of the low $q$ peaks of the pattern in c) with two rhombic symmetries. 

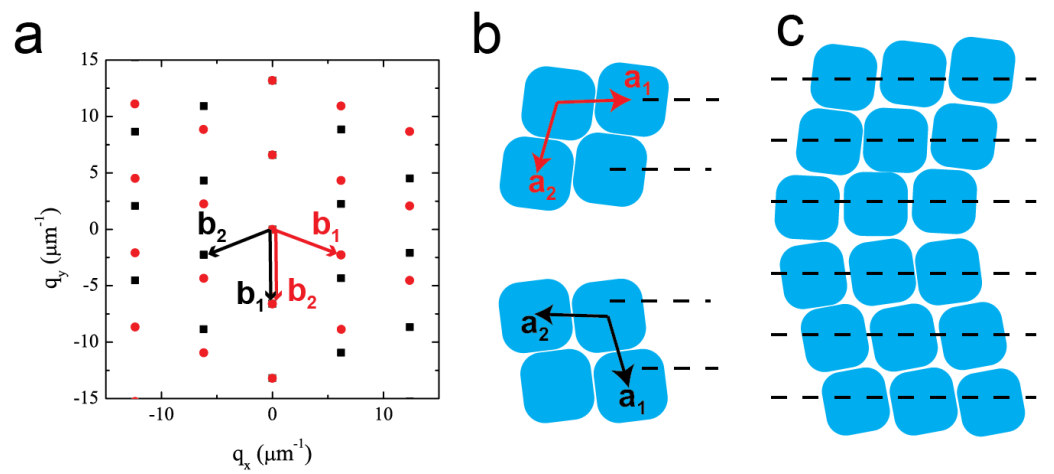

Figure 8.10. a) Reciprocal space lattice described by $b_{1}$ and $b_{2}$ for two different orientations rotated by $70^{\circ}$. b) schematic representation of both real space orientations which are obtained by a $20^{\circ}$ rotation of the cube orientation and a shift of nearest neighbors. c) Stacking of cube cause the simultaneous presence of the two structures.

neighbor via the other rounded corner. This is not a dramatic reorganization of the structure as in a perfect $\Lambda 1$-lattice this requires only a cube rotation of $20^{\circ}$. Furthermore, the fact that the reflections are smeared along the vertical direction indicates that the change in structure is continuous. This can be explained as the presence of sliding horizontal layers as the spacing of the two structures in the horizontal direction is similar. One can easily imagine a small change in the structure as illustrated in Figure 8.10.c to be the cause of the two crystal orientations. Especially when taking into account the lower osmotic pressure in this part of the sediment which will result in larger free volume for the cubes.

\subsection{Conclusions}

The crystallization of suspensions of hollow colloidal silica cubes due to sedimentation is reported. In both water and ethanol the range of the Debye screening length $\mathrm{K}^{-1}$ is found to influence the crystallization, which seems to occur only up to a critical $\kappa^{-1}$ value. Evidently, attractive forces are present between the cubes which cause gelation during sedimentation but nevertheless do not prevent later crystallization. For smaller $\kappa^{-1}$ aggregation of the cubes occurs and disordered randomly packed sediments are obtained. By elevating the $\mathrm{pH}$ with TMAH and hence increasing the cube surface charges it was found that crystallization could still occur at $\kappa^{-1}=4 \mathrm{~nm}$.

SAXS measurements of the obtained crystal phases reveal that single crystal structures with long-range translational and orientational ordering are formed in the sediments. The crystals consist of rhombic ordered layers of cubes that are aligned parallel with the capillary walls due to the large pressure exerted by the bulk sediment of the cubes. The rhombic order in the layers is found to be a consequence of the rounded corners of the cubes and can be described by the predicted densest lattice packing of superballs in $2 \mathrm{D}$, the $\Lambda_{1}$-lattice ${ }^{[17,18]}$. Rotation scans revealed that the layers are stacked with an ABA sequence. At the top of the sediments coexistence of two 
rhombic lattices is found, which differ only slightly in cube orientation and nearest neighbors and can therefore easily transform into one another.

\subsection{Outlook}

Preliminary SAXS measurement on hollow colloidal cubes of different sizes and $m$-values have shown that during sedimentation in aqueous suspensions crystallization can also occur. With the obtained knowledge about cube alignment and crystal structure these crystal structures could be evaluated. It would be interesting to see whether these structures corresponds to the predicted structure of the simulations.

\section{Acknowledgements}

Antara Pal is thanked for useful discussions and contributions to the data analysis. The Nederlandse Organisatie voor Wetenschappelijk Onderzoek (NWO) is acknowledged for the provided beam-time. Anke Leferink op Reinink and Joost Wolters are thanked for their assistance during the SAXS measurements. The whole DUBBLE team and especially Guiseppe Portale and Daniel Hermida Merino are thanked for their excellent support during the SAXS measurements.

\section{Appendix}

\section{A.8. Form factor contributions in different directions}

For the high $q$ features in the 2D SAXS patterns shown in Figure 8.11.a. good form factor fits were obtained using Eq. (6.2) for that of a shell. For the vertical peaks (Fig. 8.11.b) a shell with an inner diameter $D_{1}=1.37 \mu \mathrm{m}$ and outer diameter $D_{2}=1.47 \mu \mathrm{m}$ was found to provide a good matc. These diameters correspond to the precursor hematite cube and hollow silica cube face diagonals, $L$, as determined via Eq.(5.2). For the diagonal peaks (Fig. 8.11.c) a shell with $D_{1}=$ $1.18 \mu \mathrm{m}$ and $D_{2}=1.27 \mu \mathrm{m}$ provided the best match, which correspond to the edge lengths $D$ of the cubes (see Section 8.2.1). These form factor fits indicate that the cubes are oriented with one face against the capillary walls and the the face diagonal is alligned to the capillary length and gravity.
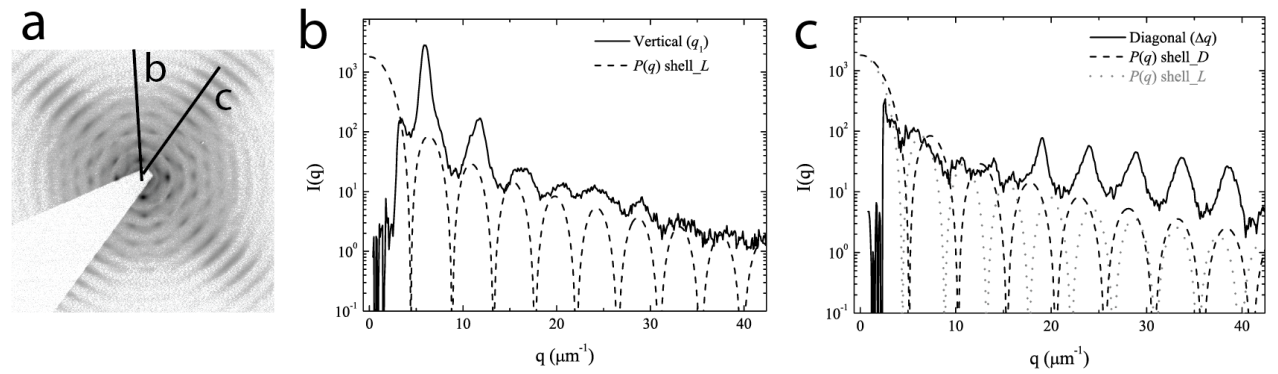

Figure 8.II. a) 2D SAXS pattern of sample $T$ in the single crystal region. b) vertical profile fitted with $P(q)_{\text {shell }}$ based on the long diagonal $L$. c) diagonal profile along high $q$ features fitted with $P(q)_{\text {shell }}$ for the edge length $D$ as well as $P(q)_{\text {shell }}$ for $L$, which have different minima. 


\section{References}

1. Y. Jiao, F. H. Stillinger and S. Torquato, Phys. Rev. E, 2009, 79, 041309.

2. R. D. Batten, F. H. Stillinger and S. Torquato, Physical Review E, 2010, 81, 1-13.

3. R. Ni, A. P. Gantapara, J. de Graaf, R. van Roij and M. Dijkstra, Soft Matter, 2012, 8, 12135-12135.

4. L. Rossi, S. Sacanna, W. T. M. Irvine, P. M. Chaikin, D. J. Pine and A. P. Philipse, Soft Matter, 2011, 7, 4139-4139.

5. L. Rossi, Colloidal Superballs, Ph.D. Thesis, Utrecht University, 2012.

6. P. N. Pusey and W. van Megen, Nature, 1986, 320, 340-342.

7. C. G. De Kruif, P. W. Rouw, J. W. Jansen and A. Vrij, J. Physique, 1985, 46, 295-308.

8. D. M. E. Thies-Weesie, Sedimentation and liquid permeation of inorganic colloids, Ph.D. Thesis, Utrecht University, 1995.

9. E. Homan, M. Konijnenburg, C. Ferrero, R. Ghosh, I. Dolbnya and W. Bras, J. Appl. Cryst., 2001, 34, 519-522.

10. A. Petukhov, J. Thijssen, D. t Hart, A. Imhof, A. van Blaaderen, I. Dolbnya, A. Snigirev, A. Moussaid and I. Snigireva, J. Appl. Cryst., 2006, 39, 137-144.

11. J. H. J. Thijssen, A. V. Petukhov, D. C. ‘t Hart, A. Imhof, C. H. M. van der Werf, R. E. I. Schropp and A. van Blaaderen, Adv. Mater., 2006, 18, 1662-1666.

12. A. Snigirev, V. Kohn, I. Snigireva and B. Lengeler, Nature, 1996, 384, 49-51.

13. V. Kohn, I. Snigireva and A. Snigirev, Opt. Commun., 2003, 216, 247-260.

14. M. Drakopoulos, A. Snigirev, I. Snigireva and J. Schilling, Appl. Phys. Lett., 2005, 86, 014102.

15. K. Zhao, R. Bruinsma and T. G. Mason, Proc. Natl. Acad. Sci. U. S. A., 2011, 108, 2684-2687.

16. Y. Zhang, F. Lu, D. van der Lelie and O. Gang, Phys. Rev. Lett., 2011, 107, 135701.

17. Y. Jiao, F. H. Stillinger and S. Torquato, Phys. Rev. Lett., 2008, 100, 245504.

18. C. Avendano and F. A. Escobedo, Soft Matter, 2012, 8, 4675-4681.

19. A. Guinier, X-Ray Diffraction in Crystal, Imperfect Crystals, and Amorphous Bodies, Dover, New York, 1994. 


\section{Chapter 9}

SeDimentary CRYSTALS of Magnetic HEMATITE CUBES AND THE INFLUENCE OF an EXTERnal MAgnetic FIELD

Abstract

The structure of spontaneously formed crystals in the sediments of colloidal hematite cubes and silica coated hematite cubes is studied using small angle $\mathrm{X}$-ray scattering (SAXS). The cube interactions were modified by dispersing the cubes in different solvents. The particles were allowed to form sediments under the simultaneous effect of the gravitational field and in a number of cases in an external magnetic field. The SAXS measurements revealed that by increasing the double layer repulsion, that opposes the magnetic and Van der Waals attractions, ordering can be induced in the sedimenting systems of the cubes. The presence of an external magnetic field during sedimentation induced the formation of a single crystal structure with long-range order, that showed different symmetries, four-fold or six-fold, close to the fluid-solid interface. 


\section{I. Introduction}

Self-assembly of colloidal particles can be directed in various ways. For example, external electric or magnetic field can change the symmetry of assembly of colloidal spheres if sufficiently strong dipole-dipole interactions are induced ${ }^{[1-3]}$. Additionally, inter-particle attractive and repulsive interactions can be adjusted to further tune the self-assembly process. An alternative route is changing the particle shapes that due to purely geometrical interactions form various phases that are related to the particles anisotropy ${ }^{[4-7]}$.

In this chapter the organization of the colloidal cubes of hematite with a permanent magnetic dipole $^{[8-11]}$ into three dimensional (3D) structures is investigated. The effect of their superball shape, surface charge and alignment in a magnetic field on the resulting self-assembled structures is investigated. Densely packed sediments of hematite cubes are formed owing to the size and the high mass density of the hematite cubes. Because the cubes are charge stabilized, interactions were adapted by changing the dispersion medium, such as water and ethanol. Moreover, the surface charge sign was changed via either the $\mathrm{pH}$ or by coating the particles with a silica shell. Furthermore, the effects of an external magnetic field were investigated using specialized magnetic setups. Cube structures were characterized by small angle X-ray scattering (SAXS) with microradian resolution that provides structural information via the structure factor peaks. Using this approach, the most favourable conditions for the self-assembly of cubes into long-range ordered structures in the sediment were identified. An external magnetic field was applied to induce particle alignment and led to the formation of a well-ordered crystal of silica coated hematite cubes that was investigated in detail. In addition the fluid-solid interface of the forming sediment was imaged in real space using Hard Transmisson X-ray Microscopy (TXM).

\subsection{Experimental}

\subsubsection{Chemicals}

Demineralized water was obtained from a Millipore system $(\mathrm{pH} \sim 5)$ and was used for all experiments unless stated otherwise. Buffers were prepared from sodium bicarbonate (p.a. Merck), sodium carbonate (p.a. Merck), sodium acetate trihydrate (Lancaster, 99\%), acetic acid (p.a. Merck), disodium hydrogen phosphate (Sigma Aldrich, >99\%) and potassium dihydrogen phosphate (p.a. Merck) dissolved in water. All chemicals were used as received.

\subsubsection{Colloidal cubes}

Several different types of hematite cubes and silica coated hematite cubes were prepared according to the protocol described in Chapter 5, Section 5.2.1 \& 5.2.2. The particles were characterized with transmission electron microscopy (TEM, Philips TECNAI 10 or 12) and their electrophoretic mobility was measured with a Malvern Zetasizer Nanoseries.

The cube edge length $D$ and the value of their shape parameter $m$, as determined from at least 100 individual particles in TEM images, are given in Table 9.1. For details on this procedure see ref. ${ }^{[11]}$ and Chapter 5, Section 5.3.2. The average cube edge lengths, $<D>$, span a size range of 

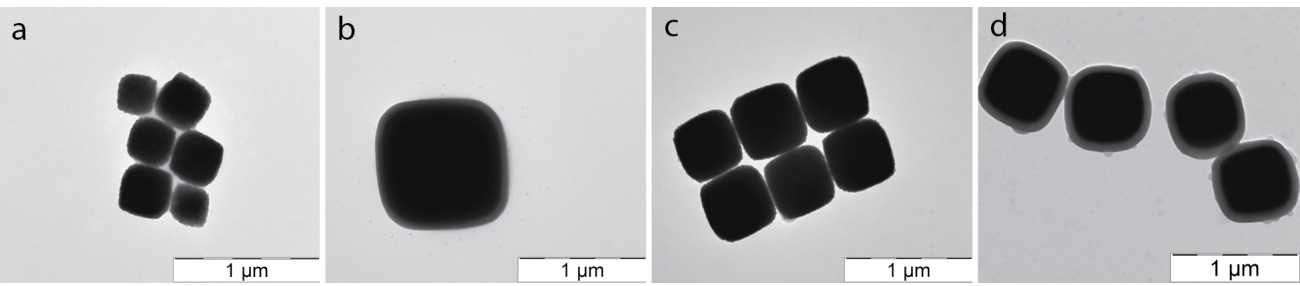

Figure 9.I. TEM images of some of the differently sized hematite cubes used for the experiments: a) $\mathrm{Cl} 5$ b) HC5_Si c) L2 and d) L2_Si. The '_Si' denotes that the cubes are coated with a silica shell which is visible as the grey layer around the darker hematite cores.

Table 9.I. Particle properties of the hematite cubes and silica coated hematite cubes.

\begin{tabular}{|c|c|c|c|c|c|}
\hline Cube & $\begin{array}{l}<D> \\
(n m)\end{array}$ & $\begin{array}{c}\sigma_{D} \\
(\%)\end{array}$ & $\begin{array}{c}<T_{S_{i O 2}}> \\
(n m)\end{array}$ & $m$ & $\begin{array}{l}L_{g-\mathrm{H} 2 \mathrm{O}} \\
(\mathrm{nm}) \\
\end{array}$ \\
\hline \multicolumn{6}{|l|}{ Hematite } \\
\hline HC15 & 269 & 17 & - & $a$ & 5006 \\
\hline L2 & 533 & 4 & - & 3.55 & 641 \\
\hline HC5 & 932 & 5 & - & $a$ & 120 \\
\hline $\mathrm{J1}$ & 1055 & 7 & - & $a$ & 83 \\
\hline C9 & 2085 & 9 & - & $a$ & 11 \\
\hline \multicolumn{6}{|c|}{ Hematite + Silica } \\
\hline C15_Si & 340 & 13 & 33 & $a$ & 2470 \\
\hline L2_Si & 652 & 7 & 59 & 3.08 & 351 \\
\hline F_Sil & 668 & 4 & 55 & 3.13 & 326 \\
\hline HC5_Si & 1028 & 4 & 48 & 3.55 & 89 \\
\hline
\end{tabular}

${ }^{a}$ for these cubes $\mathrm{m}$ was not determined.

270-2085 $\mathrm{nm}$ and their shape parameter determined from superball shape fits ranges from $m$ $=3.08$ to $m=3.55$. Figure 9.1 shows representative TEM images of some of the hematite cubes with and without a silica coating, clearly showing the superball shape.

\subsubsection{Sample Preparation}

Stable suspensions of hematite colloids are obtained by working either above or below the iso-electric point (IP) of hematite which is located around 7.5-8.5 $5^{[12]}$. The IP changes when we coat the cubes with silica, because the point of zero charge changes to 1.7-3.5, the typical values for silica. Hematite also possesses a magnetic moment that induces dipole-dipole attractions (see Chapter 5, Section 5.3.4). To obtain a stable self-assembling system of hematite cubes the Van der Waals and magnetic attraction have to be sufficiently counteracted by the double layer repulsion. To identify these conditions, the cubes were dispersed in several different solvents: water, ethanol, $200 \mathrm{mM}$ buffers; acetate buffer $(\mathrm{pH}=3.6)$, phosphate buffer $(\mathrm{pH}=5$ 
and $\mathrm{pH}=8)$ and carbonate buffer $(\mathrm{pH}=9.2$ and $\mathrm{pH}=10.8$ and $6 \mathrm{mM}$ tetramethylammonium hydroxide(TMAH) solution which serves as a surface active ion and increases the $\mathrm{pH}$.

The dispersions eith typically $5 \mathrm{wt} \%$ of cubes were placed in rectangular capillaries of $100 \times 4 \times 0.2 \mathrm{~mm}$ internal dimensions that were stored with their long axis parallel to the gravitational field. Dense sediments were obtained within 24 hours to 72 hours depending on the particle size.

\subsubsection{Magnetic field setup}

For sedimentation experiments in a homogeneous magnetic field experiment we designed and manufactured two Magnetic Field Setups. These magnetic setups consisted of six permanent magnets with the field oriented horizontally that could be adjusted between $20 \mathrm{mT}$ and 600 $\mathrm{mT}$. Freshly redispersed samples were placed in the center of the magnetic field and allowed to sediment. For this both magnetic setups were used. The advantage of the second magnetic setup was that it could be placed directly on the rotation and translation stage of beam-line BM26B with correct center-of-rotation positioning and allowed sample rotations of $\omega \pm 70^{\circ}$. Experiments were typically performed with a magnetic field strength of $25 \mathrm{mT}$ unless state otherwise.

\subsubsection{Small angle X-ray Scattering}

Detailed information about the structure in the sediments was obtained with SAXS using compound refractive lenses $(\mathrm{CRL})^{[13-15]}$ at beam-line BM26B DUBBLE ${ }^{[16-18]}$ and SAXS in combination with transmission HXRM at optics test bench ID06, ${ }^{[19,20]}$ both at the ESRF in Grenoble. The selected energy for the X-ray beam was and $13 \mathrm{keV}$ for DUBBLE and $12.2 \mathrm{keV}$ for ID06. The CCD X-ray detector used for SAXS had a pixel size of $9 \times 9 \mu \mathrm{m}$ (Photonic Science). The detector was protected from the direct X-ray beam using a wedge-shaped beam-stop at DUBBLE (2 different occasions as can be identified by the different wedge orientations) and a bar-shaped beam-stop at ID06. The HRXM detector was a high resolution CCD detector ESRF Frelon 2000 with $0.56 \times 0.56 \mu \mathrm{m}$ pixel size and dimensions of $2048 \times 2048$ pixels. The sampledetector distance was kept between 6.7 and $7.5 \mathrm{~m}$. The X-ray beam size at the sample was $500 \times 500 \mu \mathrm{m}$ at DUBBLE and $200 \times 200 \mu \mathrm{m}$ at ID06. The sediments were scanned from top to bottom with the X-ray beam perpendicular to the flat side of the capillary. Rotations scans were performed around the samples vertical axis over a range of $\omega \pm 70^{\circ}$. At $\omega=0^{\circ}$ the capillaries were oriented with their short axis $(0.2 \mathrm{~mm})$ along the $\mathrm{X}$-ray beam. 


\subsection{Results and Discussion}

\subsection{Effect of $\mathrm{pH}$ and ionic strength}

Sample appearance. All sedimentation samples were visually inspected, as the presence of cube adsorption to the capillary glass walls or optical Bragg reflections are first indications of the particle charge sign or the presence of ordered structures. Figure 9.2 shows photographs of the sediments of three different hematite cubes C15, L2 and J1. Note that the color changes with hematite cube size, from bright orange for C15 with $D=269 \mathrm{~nm}$ to purple for J1 with $D=1055 \mathrm{~nm}$.

Figure 9.2.a and 9.2.b show that the cubes dispersed in water $(\mathrm{pH} 5)$ tend to adsorb on the capillary walls while at elevated $\mathrm{pH}(\sim 9)$ by the addition of TMAH the cubes form sediments with a straight interface. This difference in behavior is the result of the difference in $\mathrm{pH}$ that changes the charge sign on the hematite cube surface above and below the isoelectric point (IP). For instance, for the $\mathrm{L} 2$ cubes dispersed in Millipore water $(\mathrm{pH} \sim 5)$ the zetapotential is $\zeta=38.2 \mathrm{mV}$ and changes to $\zeta=-75.0 \mathrm{mV}$ in the TMAH solution. In the TMAH sample also weakly visible Bragg reflections were observed indicating that further from the IP ordered structures form, although it has to be noted that $\mathrm{TMA}^{+}$is a surface active ion. The $\mathrm{pH}$ also has an effect on the surface charge density as can be observed for the large J1 cubes that dispersed at low $\mathrm{pH} \sim 3$ show strong Bragg reflections (Fig. 9.2.c). These observations already indicates that the double layer repulsion has a clear influence on the self-assembly of the cubes in the sediments.

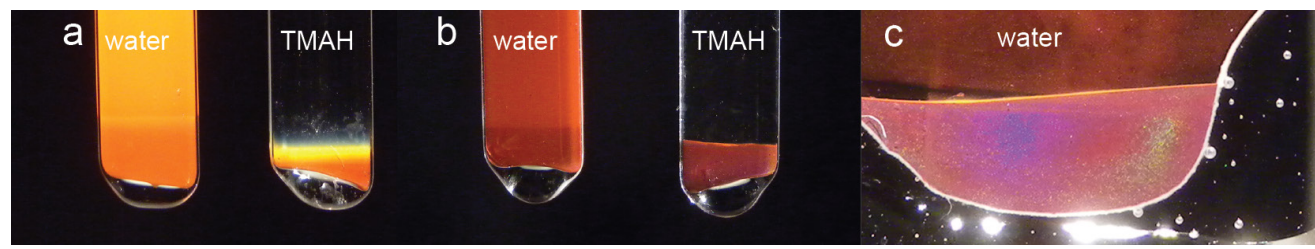

Figure 9.2. Photographs of the sediments obtained in dispersions of the different hematite cubes a) $\mathrm{CI} 5$ b) $\mathrm{L} 2$ in water and in water with $\mathrm{pH} 9$ by the addition of $\mathrm{TMAH}$ and c) $\mathrm{JI}$ in water $\mathrm{pH}$. Note the colour change of the hematite from orange to purple with increasing particle size and the difference in particle-glass interactions, as in water particles are adsorbed onto the glass wall and in TMAH solution they are not.

SAXS analysis. In the 2D SAXS patterns of the hematite cubes sediments in different solvents interesting features are observed that reveal short-range as well as long-range order. It has to be noted that these scattering patterns are of course influenced both by the form factor and structure factor. In order to disentangle these effects we would have to divide out the cube form factor. However, the non-spherical shape couples the orientational and positional interparticle correlations making the form and structure factor separation ambiguous. Moreover, as discussed in Chapter 5, Section 5.3.4 the cubes form clusters due to their magnetic interactions and rapid sedimentation occurs that makes measurements of single cube form factors difficult. Therefore, the analysis is limited to structure factor peaks, which can be clearly distinguished in most of the SAXS patterns. 

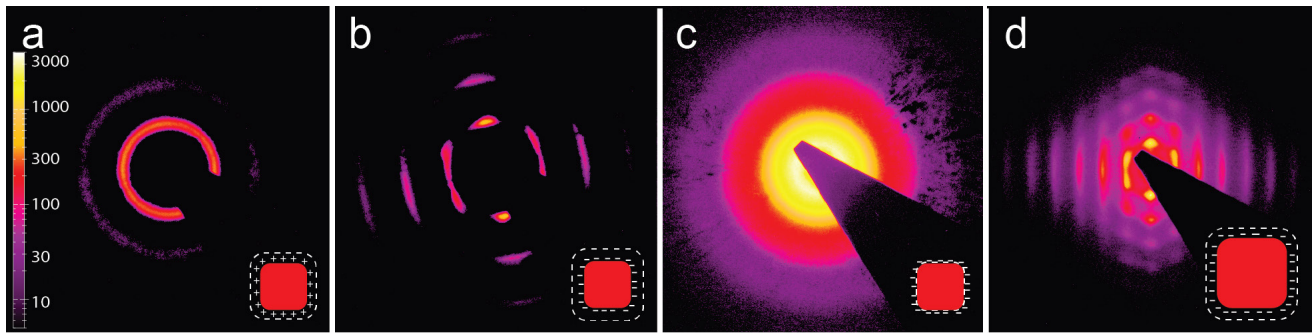

Figure 9.3. 2D SAXS patterns of the sediment of hematite cubes $L 2$ with $D=533 \mathrm{~nm}$ in different solvents; a) Milipore water b) 6 mM TMAH, pH 9and c) 200 mM phosphate buffer, pH 9.2 ; d) 2D SAXS pattern of cubes $\mathrm{JI}$ with $\mathrm{D}=1055 \mathrm{~nm}$ at $\mathrm{pH} \sim 3$. Single crystal structures are seen for L2 and JI at high surface charge. Insets show schematic representation of the cube and increase or decrease of the Debye screening length. The black wedge is the shadow of the beamstop to protect the detector from the direct X-ray beam.

In the height scans of the typically $5 \mathrm{~mm}$ high sediments, ordered regions were often observed at the top region of a few $\mathrm{mm}$. The bottom parts of the sediments usually showed disordered regions, due to the gravitational compression exerted by the total mass of the cubes above causing kinetic arrest. In samples containing polydisperse particles size fractionation was also observed. Figure 9.3 shows typical 2D SAXS patterns of the sediments of L2 cubes with $D=533 \mathrm{~nm}$ dispersed in different solvent. The patterns were taken at $1 \mathrm{~mm}$ from the top of the sediments. For hematite cubes in demineralized water (Fig. 9.3.a) a strong structure factor peak can be observed at $q=0.0110 \mathrm{~nm}^{-1}$ on top of the form factor, indicating that the hematite cubes positions are locally correlated with a $d$ spacing of $571 \mathrm{~nm}$. The presence of a ring indicates that the sample is very polycrystalline and order extends over roughly 5-10 cube lengths. The shortrange order can be explained by the magnetic dipole-dipole interactions and the low surface charge density the hematite particles carry in water ${ }^{[21]}$. Once in the sediment the surface charge is not high enough to compensate the gravitational compression causing kinetic arrest and an inability for neighboring crystals to reorient.

For cubes that are dispersed in $6 \mathrm{mM}$ TMAH in water at $\mathrm{pH} 9$, the surface charge sign is reversed and due to the salt content the Debye Length $\kappa^{-1}$ is reduced to $4 \mathrm{~nm}$. However, it is known that the $\left[\left(\mathrm{CH}_{3}\right)_{4} \mathrm{~N}\right]^{+}$cations adsorb on the hematite surface and provides additional stabilization $^{[21,22]}$. The SAXS pattern shows the presence of a large single crystal domain (Fig.9.3.b) indicating that under these conditions the cubes have assembled into an ordered structure. The observed Bragg spots indicate that the single crystal orientation is extended over at least $500 \times 500 \mu \mathrm{m}$ (the X-ray beam size). The two peaks in the vertical direction at $q=0.0111$ $\mathrm{nm}^{-1}$ in Figure 9.3.b indicate that planes have formed perpendicular to the direction of the gravitational field. Their $d$ spacing of $566 \mathrm{~nm}$ is close to $D=533 \mathrm{~nm}$ of the cubes and can be explained by alignment of the cube faces to each other and to the flat walls of the capillary. Two peaks in the horizontal direction in Figure 9.3.b, which are smeared out vertically $(0.0115$ $<\mathrm{q}<0.0117 \mathrm{~nm}^{-1}$ ), indicate that the layers of cubes are randomly shifted on top of each other 
resulting in periodic distances in the range of 546 - $537 \mathrm{~nm}$. Hexagonal or brick-wall-like stacking, with the cubes in a layer above positioned in the niches formed by two cubes in the layer below, could explain the hexagonal symmetry. However, due to the rounded corners of the cubes, also a rhombic stacking of the cubes could be achieved with similar spacing.

Dispersions cubes at pH 9.2 and thus negatively charged but at high salt content in $200 \mathrm{mM}$ buffer showed the formation of clumps during preparation followed by fast sedimentation. Also the final sediment height was found to be larger than for cube dispersion with the same initial $\mathrm{wt} \%$ at lower salt concentration. Clearly aggregation of the cubes occurred as expected by the decrease of the Debye length which at $200 \mathrm{mM}^{-1} \mathrm{k}^{-1}<1 \mathrm{~nm}$. Figure 9.3.c shows the SAXS pattern of this sample which possesses no clear structure factor peaks. This shows that little correlation between cube positions is present. The absence of long-range positional order is attributed to the aggregation of the cubes into disordered clusters due to the short $\kappa^{-1}$ and the magnetic attractions. The aggregates will sediment into loosely packed, low density structures. Evidently, $\mathrm{pH}$ conditions far below or above the IP and at low salt concentration are needed for ordering to occur in the bare hematite cube sediments.

That conditions at low $\mathrm{pH}$ and no added salt allow self-assembly of the cubes into ordered structures is confirmed by measurements of the sediment of J1 cubes. Figure 9.3.d shows the 2D SAXS pattern of the sample shown in Fig.9.2.d that shows similar hexagonal peaks as the L2 cubes in TMAH. Surprisingly, these large cubes can form a similar ordered structure despite having a high sedimentation speed of $0.3 \mu \mathrm{m} / \mathrm{s}$ (determined from the full sedimentation over $8 \mathrm{~cm}$ in 7 hours). For the biggest cubes C9 with $D=2085 \mathrm{~nm}$ ordered structures were never observed, indicating that for this size sedimentation is too fast to allow organization in the sediment.

Thus, by tuning the hematite surface charge and Debye length $\kappa^{-1}$ the formation of long-range crystal structures of cubes can be controlled during sedimentation. The obtained layered crystal structure is induced by the cube shape - flat faces align with each other and the capillary walls - and the attractive magnetic dipole-dipole interactions between the cubes.

We realize that the explanations of the various structures presented above, still lack a quantitative basis. Though we know Debye lengths and have an estimate of the zeta potential the details of the double layer repulsion between the cubes are still unknown. There is no expression for the anisotropic DLVO potential between two cubes; in addition the magnitude and direction of the magnetic moment is still under study ${ }^{[23]}$. Computer simulation of sedimentary crystals of cubes would be very helpful in further quantitatively explaining our findings.

\subsubsection{Effect of silica coating}

Sample appearance. The silica coating on the hematite cubes changes the surface chemistry of the cubes. As a result the cubes have a negative surface charge in water, for instance L2_Si has a zetapotential of $\zeta=-49 \mathrm{mV}$ in water and $\zeta=-44 \mathrm{mV}$ in ethanol. Figure 9.4 shows photographs of sediments of silica coated hematite cubes in water and ethanol in which their behavior is quite different. The cubes dispersed in water have slightly adsorbed to the glass wall, while those 

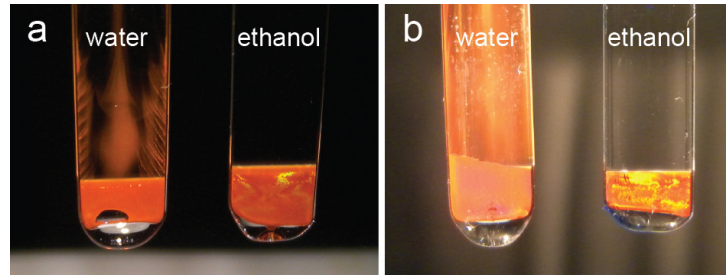

Figure 9.4. Photographs of the sediments of silica coated hematite cubes a) CI5_Si and b) L2_Si dispersed in water and ethanol. Cubes dispersed in water adsorb slightly to the glass wall, while strong Bragg reflections are visible for the cubes dispersed in ethanol, indicating ordering in the sediments.

dispersed in ethanol do not show signs of adsorption and reveal distinct Bragg reflections in the sediment, indicating ordering of the cubes.

SAXS analysis. Figure 9.5.a and 9.5.b show the 2D SAXS patterns of the silica coated hematite cubes L2_Si in water and ethanol. In the pattern of the sediment in water a strong but broad structure factor ring is observed, indicating local short-range order of the cubes. This is similar to the structure observed for the non-coated hematite cubes in water (Fig. 9.3.a). Figure 9.5.b shows the scattering pattern of the sediment in ethanol which shows distinct peaks indicating the presence of a long-range ordered structure.

The profile plots of the 2D SAXS patterns of L2_Si are shown in Figure 9.5.c. The positions of the first structure factor peaks are locates in water at $q=0.0113 \mathrm{~nm}^{-1}$ indicating an intercube spacing of $d=556 \mathrm{~nm}$ and in ethanol at $q=0.00922 \mathrm{~nm}^{-1}$ with $d=681 \mathrm{~nm}$. Clearly, the difference in structure formed by the cubes in water and ethanol is caused by a difference in inter-cube spacing. This could be related to the Debye screening length $\kappa^{-1}$, which is dependent on the dielectric constant, $\varepsilon_{\mathrm{r}}$, and ionic strength, $I$, of the solvent. To both solvents no salt was added and with an estimated $I \sim 10^{-5} \mathrm{M}$ the Debye lengths in water with $\varepsilon_{\mathrm{r}}=80$ is about $\kappa^{-1}=97 \mathrm{~nm}$ and for ethanol with $\varepsilon_{\mathrm{r}}=20$ we estimate to be $\kappa^{-1}=49 \mathrm{~nm}$. However, the presence of these Debye lengths would result in the opposite $d$-spacings and hence these $\kappa^{-1}$-values are not
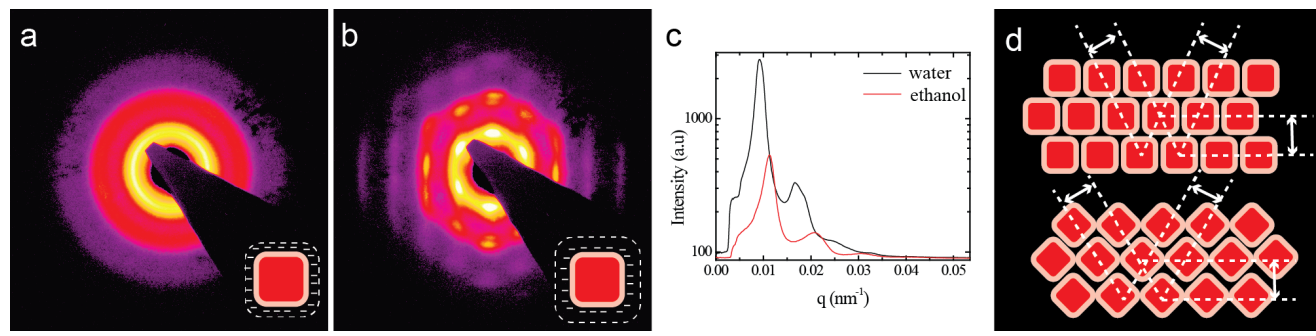

Figure 9.5. 2D SAXS patterns of silica coated hematite cubes L2_Si with $D=652 \mathrm{~nm}$ in a) water and b) ethanol. In water only short-range local order is observed, while in ethanol long-range ordered single crystal structures are obtained, due to higher screening of the cubes double layer repulsion in water. c) Profile plots of the SAXS patterns shown in a) and b). d) A schematic representation of the layered structure, showing the origin of the hexagonal peaks. 
correct. The difference probably originates from the different stability of porous silica in water and ethanol. It is known that the porous silica can dissolve in water into $\mathrm{Si}(\mathrm{OH})_{4}{ }^{[24]}$ which will increase $I$ and hence decrease $\kappa^{-1}$. This decrease in stability would also explain the observed adsorption of the particles on the glass wall.

The silica coated cubes in ethanol have clearly formed a similar crystal structure as the hematite cubes in TMAH. However, the order in the silica coated cube sediment is found to possess much more positional correlation between the layers as seen by the appearance of $3^{\text {rd }}$ order Bragg peaks. We attribute this increase in order to a decrease in magnetic dipoledipole attractions between the cubes, caused by the silica shell. As a result the cubes have more freedom to reach an optimal lattice position. The intensity of the two horizontal peaks is slightly smeared, indicating that slight deviations from the optimal positions occur. Figure 9.5.d shows two schematic representations of the structure consisting of hexagonal stacked layers of cubes. Based on the position of the Bragg peaks alone and the fact that the peaks are smeared we cannot distinguish between the two structures for now. The cubic shape of the cubes is shown because we do expect the cubes to be are aligned with their faces against the glass wall. The cubes in a layer on top are arranged into a niche of the layer below. The dashed lines indicates the periodic hexagonal order between the layers that produces the Bragg peaks.

\subsubsection{Effect of cube size}

To determine whether the observed crystal structure is a result of the cube shape and the magnetic inter-cube interactions, the effect of cube size was also investigated. Simulations and experiments have shown a correlation between the superball parameter $m$, which changes with cube size and coating ${ }^{[11]}$, and the expected crystal structure ${ }^{[25-28]}$.

Therefore, several hematite cubes were coated with silica and dispersed in ethanol. Similar structures were observed in the sediments of smaller and larger cubes than L2_Si. Figure 9.6 shows the 2D SAXS patterns of the sediments of C15_Si, $D=340 \mathrm{~nm}$ (Fig. 9.6.a) F_Si1, D=667 nm (Fig. 9.6.b) and HC5_Si, D = 1028 nm (Fig. 9.6.c). For the F_Sil cubes, of similar size as
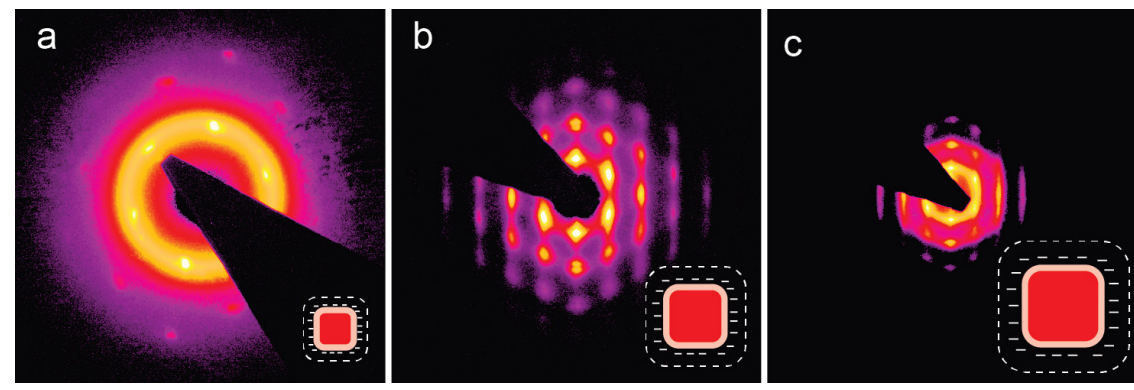

Figure 9.6. 2D SAXS of the sediments of silica coated hematite cubes of different sizes (A) CI5_Si with $D=340 \mathrm{~nm}$, (B) F_Sil with $D=667 \mathrm{~nm}(\mathrm{C}) \mathrm{HC5}$ _Si with $D=1028 \mathrm{~nm}$. All patterns shown distinct hexagonal Bragg peaks indicating layered ordering with a brick-wall-like layered stacking induced by the cube shape. 
L2_Si, a strikingly similar pattern is observed and hence the same crystal structure is formed. It is also clear that for cubes of much smaller size C15_Si, and much larger, HC5_Si, the same hexagonal order can be observed. For the smaller cubes less order is found (Fig. 9.6.a) which is probably the result of the high polydispersity of $13 \%$, which is known to hinder crystallization. In addition, for the larger cubes, the faster sedimentation is probably the cause of the lower long-range order. Evidently, the cube shape and dipole-dipole interactions determine the crystal structure as for all sizes a similar layered crystal structure is obtained. However, to determine to which extent the shape parameter $m$ plays a role more detailed investigations are needed.

\subsubsection{Effect of an external magnetic field}

Because the hematite cubes possess a permanent magnetic dipole, we explored inducing directionality into the sediments of the hematite cubes with an external magnetic field. It was found that the best results were obtained when the sediments were redispersed using manual shaking and ultrasonic treatment for $\sim 15 \mathrm{~min}$, after which the particles were allowed to settle in a homogeneous magnetic field of $25 \mathrm{mT}$ over a period of 12 to 24 hours before the SAXS measurements. This process allows the cubes to align their magnetic moment to the magnetic field during sedimentation.

Hematite cubes dispersed in $6 \mathrm{mM}$ TMAH in water and allowed to settle in an applied magnetic field did not form crystalline structures. The 2D SAXS patterns of these sediments showed only a cubic form factor and no Bragg peaks were observed (see appendix A.9.2. for more details). The $2 \mathrm{D}$ form factor did indicate that all cubes acquired the same orientation in the magnetic field. However, the lack of Bragg peaks suggests that under these conditions the cubes get jammed during sedimentation. The magnetic field probably induces strong intercube attractions inducing string and cluster formation that sediment faster than single particles.

It has previously been shown that silica coated hematite cubes in ethanol are stable in an applied magnetic field ${ }^{[11]}$. Rossi et al. showed that at low volume fractions string formation occurs and at higher concentrations large square ordered rectangular $2 \mathrm{D}$ domains can form ${ }^{[11,29]}$. However, as optical light microscopy was used these studies were limited to single layers of cubes and thus only analysis of 2D structures. Due to the high penetration depth of the X-rays, the $200 \mu \mathrm{m}$ thick samples can easily be studied with a combination of SAXS and HRXM. The beam-size of the used SAXS setup at ID06 was even $200 \times 200 \mu \mathrm{m}$ allowing a more detailed investigation of the sediments than with the setup at DUBBLE.

Full scans of the sediments of silica coated hematite cubes obtained with a magnetic field of $25 \mathrm{mT}$ revealed large single-crystalline structures with long-range order in the sediment. Furthermore, an interesting structural change was observed at the fluid-solid interface. Figure 9.7 shows three 2D SAXS patterns around the fluid-solid interface at $z=0$ of the sediment of L2_Si (for C15_Si and F_Sil see Appendix A.9.3). An isotropic pattern is obtained just above the sediment, $z=0.3$ (Fig 9.7.a), while clear Bragg peaks with four-fold (Fig. 9.7.b) and six-fold (Fig. 9.7.c) symmetry are observed in the two patterns at and below the interface at $z=-0.1 \mathrm{~mm}$ and $z=-0.9 \mathrm{~mm}$, respectively. Figure 9.7.d-g shows the HRXM images of the interface. The 


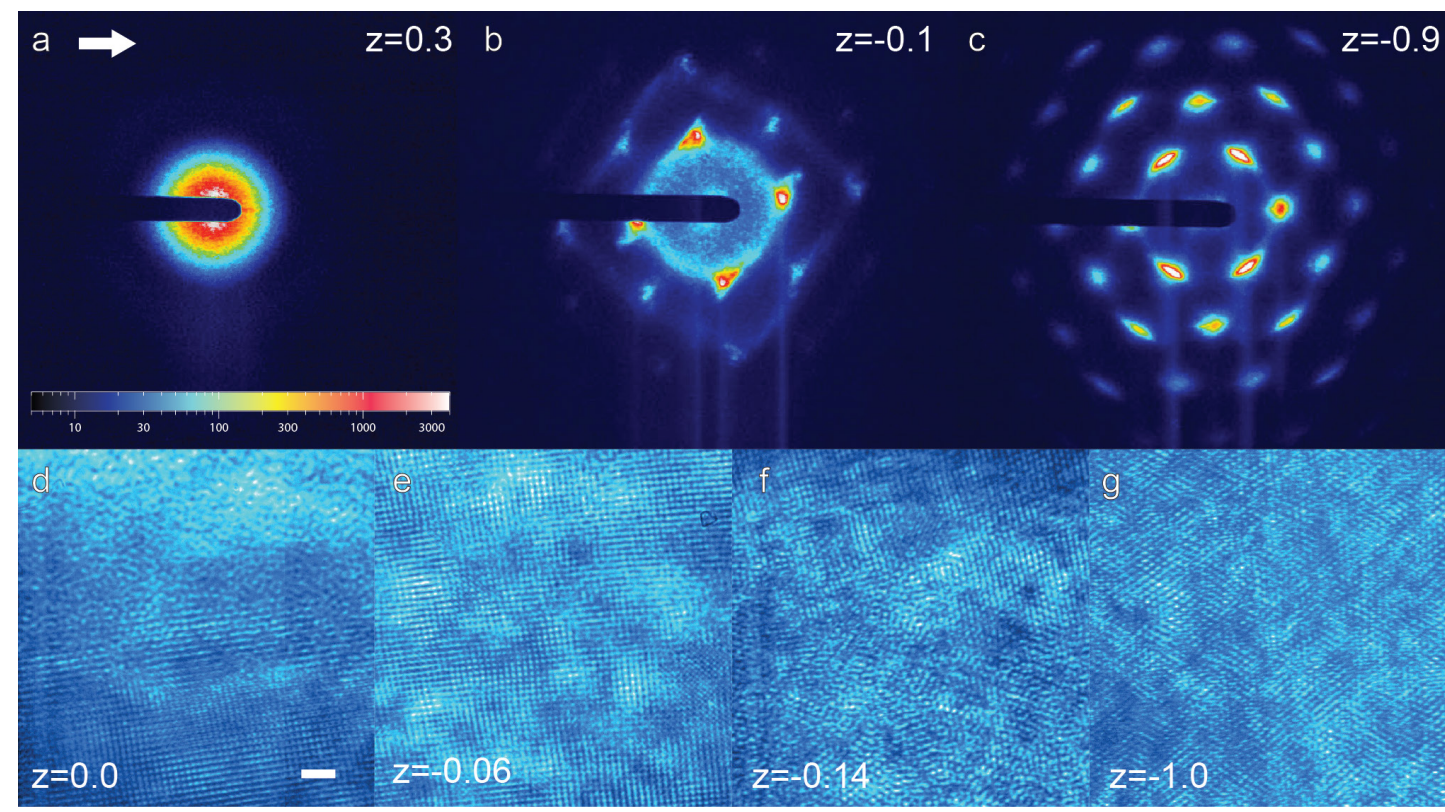

Figure 9.7. Fig. 7. SAXS patterns $(a, b, c)$ and HXRM images $(d, e, f, g)$ of the sediment of L2_Si cubes with $D=652 \mathrm{~nm}$ in a $25 \mathrm{mT}$ magnetic field after 12 hours, taken around the fluid-solid interface, $\mathrm{z}=0 \mathrm{~mm}$, of the sediment. Different structures are formed at these positions with rectangular and hexagonal periodicity perpendicular to the X-ray beam. Large white arrow in a) indicates direction of B-field, scale bar in d) is $3 \mu \mathrm{m}$.

transition region from a fluid region to an ordered region with a slightly tilted square structure can be clearly seen in Figure 9.7.d. This square structure shows very strong resemblance to the square structures observed with light microscopy ${ }^{[11]}$. However, here the image is a transmission of $\sim 300$ cubes that have evidently ordered perfectly into a structure with fourfold symmetry along the X-ray beam. From the HRXM images the height of the square region was determined to be around $100 \mu \mathrm{m}$; after that the order disappears (Fig.9.7.f) and shifts to hexagonal lower in the sediment (Fig. 9.7.g). The formation of the sediment could be followed over time and the region with square ordering was found to always remain around $100 \mu \mathrm{m}$ high and persisted over the full width, $4 \mathrm{~mm}$, of the capillary.

To understand what is happening at the interface SAXS measurements were performed of the sediment at the regions with four-fold and six fold symmetry while rotating the sample around its vertical axis over a range of $\omega= \pm 70^{\circ}$ with $1^{\circ}$ or $5^{\circ}$ step. These measurements were performed with a $500 \times 500 \mu \mathrm{m}$ beam at DUBBLE and thus contributions from both symmetry regions were obtained in the patterns.

SAXS patterns at rotation angles corresponding to high-symmetry directions are shown in Figure 9.8; here the strongest Bragg peaks were observed. In the 2D SAXS patterns of the top region of the sediment at $z=-0.1 \mathrm{~mm}$ Bragg spots with four-fold symmetry can be observed at 


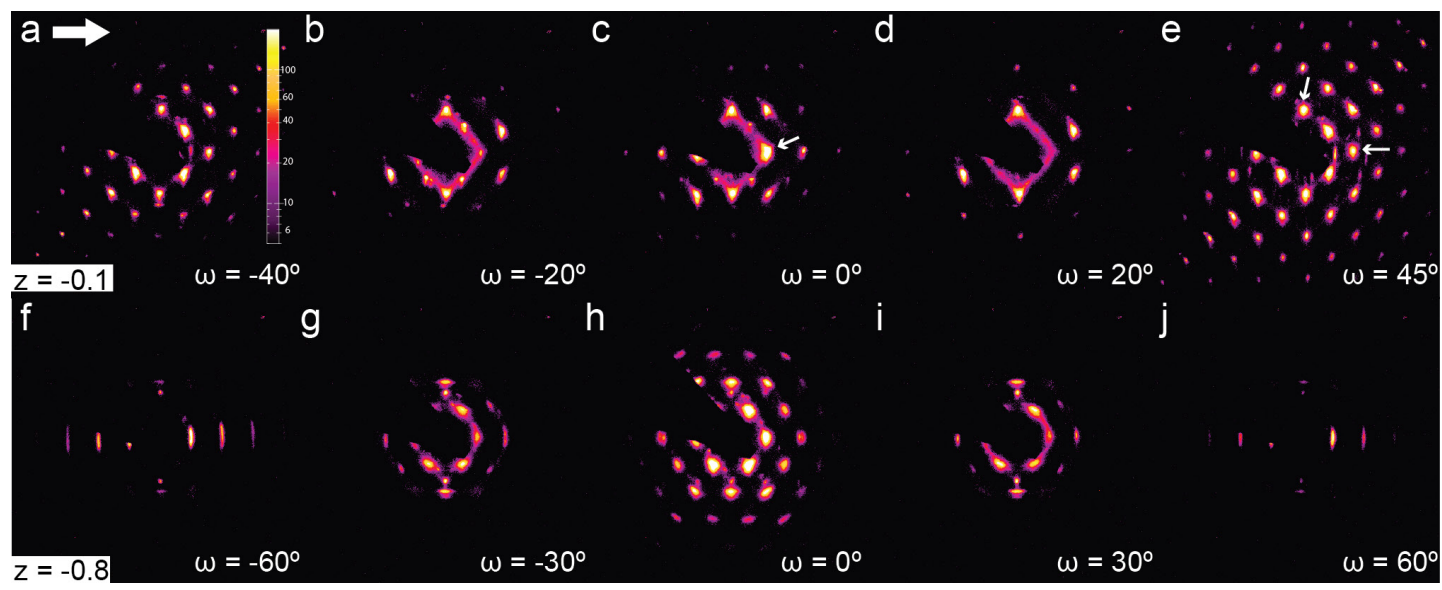

Figure 9.8. SAXS patterns of L2_Si in a $25 \mathrm{mT}$ magnetic field obtained at different rotations $\omega$ of the sample around its vertical axis; (a-e) for the top of the sediment at $z=-0.1 \mathrm{~mm}$, large white arrow in a) indicates direction of the B-field and ( $f-j)$ for the middle of the sediment at $z=-0.8 \mathrm{~mm}$. In the top of the sediment strong Bragg peaks with four-fold symmetry symmetry are visible up to high orders, indicated a single crystal structure. In the lower part peaks with six-fold symmetry are observed up to $\omega= \pm 35^{\circ}$. For $\omega>35^{\circ}$ smeared peaks are present, indicating this structure is less ordered.

rotation angles, $\omega=-40^{\circ}, 0^{\circ}$ and $45^{\circ}$, indicating a cubic-type crystal lattice. At $-40^{\circ}$ and $45^{\circ}$ the Bragg peaks are even visible up to the 10th order (shown in Appendix A.9.5) indicating that this structure possess true long-range order. In the six-fold symmetry region at $z=-0.8$, the strong Bragg spots with six-fold symmetry are observed up to $\omega=35^{\circ}$. For $\omega>35^{\circ}$ smeared peaks in the horizontal direction can be observed together with some weak contributions of the Bragg spots of the cubic region. The structure lower in the sediment thus possesses less order than the cubic structure at the interface. Clearly, the gravitational compression must play an important role here.

To determine the crystal lattice present in the cube sediments first the positions of the Bragg peaks were determined for the top region of the sediment. The two vertical peaks observed in the patterns at $q=0.010 \mathrm{~nm}^{-1}$ were found to be present for each rotation $\omega$, indicated with the top arrow in Fig 9.8.e. This shows that there are crystal lattice planes, spaced by $486 \mathrm{~nm}$ oriented perpendicular to the beam and parallel to the field. The additional horizontal peaks (right arrow Fig 5.8.e) at $q=0.0147 \mathrm{~nm}^{-1}$ at $\omega=-40^{\circ}$ and $q=0.0149 \mathrm{~nm}^{-1}$ at $\omega=45^{\circ}$, show that cubic lattice planes are present with a slightly different spacing of 426 and $423 \mathrm{~nm}$. At $\omega=0^{\circ}$ the horizontal peak is located at $q=0.0111 \mathrm{~nm}^{-1}$ giving a lattice spacing of $566 \mathrm{~nm}$. From the symmetry the structure can be identified as an FCC-like structure with the [100] projections (Fig. 9.8.a,e) and [110] (Fig 9.8.c) seen over $90^{\circ}$ of rotation. However, the rotation between the [100] projections was found to be only $85^{\circ}$ and therefore the structure is identified as a Base Centered Monoclinic (BCM) lattice, with lattice parameters of $a=846 \mathrm{~nm}, b=852 \mathrm{~nm}$ and $c=972 \mathrm{~nm}$ with $\alpha=\beta=90^{\circ}$ and $\gamma=85^{\circ}$. 


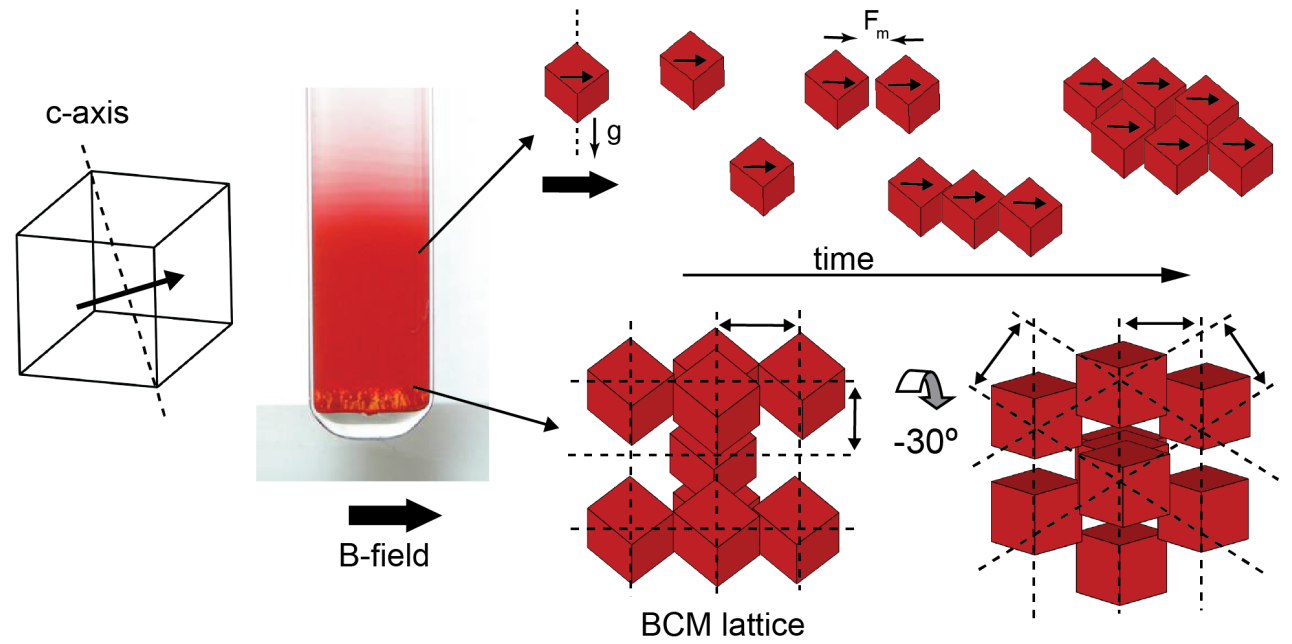

Figure 9.9. Schematic representation of the self-assembly process of the silica coated hematite cubes settling in a magnetic field. The magnetic dipole is oriented perpendicular to the c-axis (long diagonal) of the cube. During sedimentation the cubes align their dipole moment along the field and form small clusters. These clusters assemble into a close-packed BCM lattice that rotates upon gravitational compression due to alignment of the cube faces with the capillary walls.

Simulations of the optimal packings of superball shaped particles have also predicted FCCtype lattices, namely the $\mathrm{C}_{0}$ and $\mathrm{C}_{1}$ lattices ${ }^{[25]}$ and a sheared FCC or sheared SC for parallel rounded cubes ${ }^{[28]}$. The experimental BCM structure was therefore compared to these crystal structures. For this we calculated the expected BCM lattice parameters, that are also present in an FCC-type lattice in 3 orientations, for $m=3.08$. We could not find a fit of our experimental BCM lattice with any of the calculated simulation BCM lattices (see appendix A.9.6). This can be explained by the fact that in the simulations only excluded volume interactions were taken into account, while long-range repulsive forces and dipole-dipole interactions play an important role here.

The orientation of the cubes on a lattice position of the BCM lattice could not yet be determined from these measurements. However, earlier investigations of the internal cube structure have shown that the magnetic dipole should be oriented perpendicular to a long diagonal of the cube $^{[11,30]}$. However, the exact orientation and hence the orientational alignment of a cube in a magnetic field has not been established yet. It is, however, clear that the spacing between the horizontally arranged layers (aligned parallel to the magnetic field) is larger than that of the vertically arranged layers and thus we conclude that the long-diagonal of the cubes is most likely oriented vertically and thus perpendicular to the magnetic field.

The crystal structure lower in the sediment was found to correspond to the identified BCM lattice above but with a $30^{\circ}$ rotation backward or forward along the X-ray beam at $\omega=0^{\circ}$. This crystal structure is explained as follows. The cubes are aligned with their long diagonal perpendicular to the field; however, as seen for the crystals in only a gravitational field, the cubes 
preferably orient with their flat faces parallel to the capillary walls. In addition the total buoyant mass of the crystal at the fluid-solid interface is exerting a large pressure on the sediment below. Apparently, only $100 \mu \mathrm{m}$ of sediment is enough to induce the high pressure needed to overcome the magnetic dipole alignment.

To summarize, the alignment of hematite cubes in a magnetic field causes the formation of a BCM crystal lattice which is shown schematically in Figure 9.9.. First, the randomly oriented cubes align their magnetic dipole moment in the applied magnetic field. Then, flexible string clusters of cubes form and due to sedimentation a close-packed single crystal is formed, in which the cubes are aligned with their magnetic dipole along the external magnetic field. As the sediment increases by addition of cubes due to sedimentation, more and more pressure is exerted on the crystal which causes the cubes to reorient such that their flat face is parallel to the capillary wall. As a result the BCM crystal is compressed causing a slight loss of positional correlations of the cubes.

\subsection{Conclusions}

Self-assembly of colloidal hematite cubes in sedimentary crystals can be controlled by a number of factors, such as solvent, coating, size and external field. The mechanism governing the self-assembly is an interplay between the cube shape, the magnetic dipole-dipole attraction and double layer repulsion. Short-range ordered structures were obtained for hematite cubes in water, while addition of TMAH was found to induce the formation of long-range ordered crystal structures. The crystals were found to possess layers of cubes related to the alignment of the cube faces. For samples at high salt concentration that reduces the Debye length aggregation was observed as expected. Silica coating of the hematite cubes and dispersing them in ethanol was found to increase the double layer repulsion between the cubes and led to the formation of crystal structures with long-range order. The cube size was found to have a small effect on the sedimentary crystal formation as the same crystal structures were obtained.

For silica coated hematite cubes it was shown that an external magnetic field induced the formation of a single crystal structure with a BCM lattice. In the first $100 \mu \mathrm{m}$ of the sediment the cubes were found to have aligned their magnetic dipole moment along the field. Lower in the sediment the crystal structure was found to be compressed under its own buoyant mass causing the cubes to orient with their flat sides aligned to the capillary walls and rotating the crystal structure. The observed crystal did not correspond to the crystal lattices predicted by simulations for hard superballs ${ }^{[25-28]}$, which is most likely caused by the presence of the dipoledipole interactions and long-range repulsion between the hematite cubes. 


\section{Acknowledgements}

Dmytro Byelov and Laura Rossi are thanked for their collaboration on this project and the many discussions and useful suggestions for this chapter. The Netherlands Organization for Scientific Research (NWO) and the European Synchrotron Radiation Facility (ESRF) in Grenoble are thanked for the provided beam-time. G. Portale and D. Detolenaire from BM26 and A. Snigirev, I. Snigireva, C. Detlefs and T. Roth from ID06 are thanked for their support during the experiments. For their assistance during the measurements Jan Hilhorst, Anke Leferink op Reinink and Mark Vis are thanked. For their contributions to the particle syntheses Sonja Castillo, Vera Meester, Lisette Pompe, Julius de Folter and Fabian Hagemans are thanked. Part of this work is reproduced by permission of The Royal Society of Chemistry (RSC) [31]. 


\section{Appendices}

\section{A.9.I. Effect of different buffers on L2 sediments}

The hematite cubes were dispersed in different buffers and solvents. Figure 9.10.(a-c) shows the additional SAXS patterns obtained in three other buffers of $200 \mathrm{mM}$ salt with $\mathrm{pH} 5, \mathrm{pH} 8$ and PH 10.8. These all show a similar pattern as that obtained at pH 9.2 of $200 \mathrm{mM}$. Figure 9.10.d. shows the profile plot of all patterns are shown. The profiles of the sediments of the cubes in water and $\mathrm{pH} 9 \mathrm{TMAH}$ show distinct structure factor peaks on a strong form factor, while the other buffers shown only form factor contributions.

\section{A.9.2 Effect of magnetic field on hematite cubes}

Figure 9.11 shows the 2D SAXS patterns of the sediment of hematite cubes dispersed in $6 \mathrm{mM}$ TMAH in water formed in an applied magnetic field of $25 \mathrm{mT}$ over 24 hours. A mixed pattern of the structure factor and form factor are seen here, especially in Figure 9.11.a. The magnetic field causes the particles to have the same orientation during sedimentation, resulting in a specific cubic form factor. On top a structure factor peak can be seen, however, due to the lack of clear Bragg peaks it is clear that there is no long-range order present. This is most likely due to stronger induced magnetic attractions between the cubes, causing irreversible aggregation into clusters that prevent the particles to form periodic structures.

\section{A.9.3 Differently sized cubes in a magnetic field}

Figure 9.12 shows the 2D $\mu$ rad-XRD patterns for C15_Si (Fig. 9.12.a) and F_Sil (Fig.9.12.b) settled in a $25 \mathrm{mT}$ magnetic field. Here also a single crystal with rectangular order is present at the fluid-solid interface. The magnetic field clearly induces strong magnetic dipole-dipole interactions between the cubes, resulting in similar crystal lattice structures with their layers aligned along the magnetic field. In addition, the silica coating prevents the particles from forming aggregates as seen for the hematite cubes (Fig.9.11.)

\section{A.9.4. Long-range order of magnetic field induced structure}

Figure 9.12.c shows the 2D $\mu$ rad-XRD pattern of Figure 9.8.e with longer exposure time, showing the presence of Bragg peaks up to the $10^{\text {th }}$ order. 

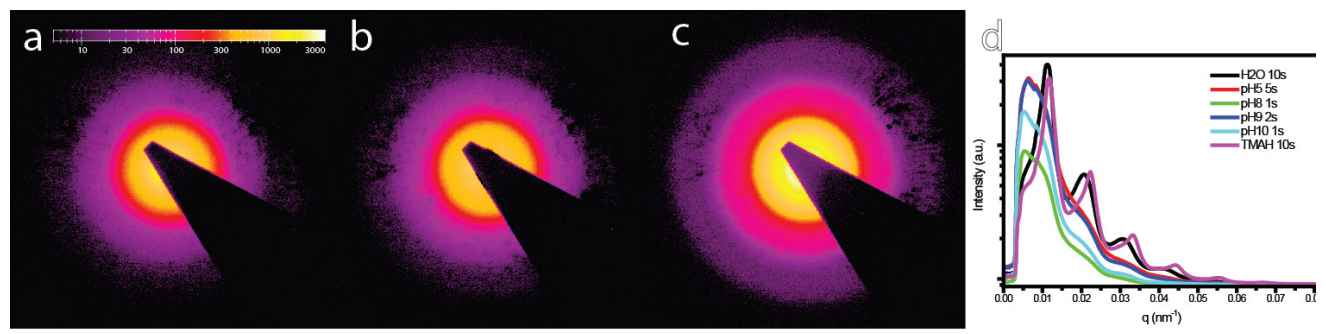

Figure 9.10. 2D SAXS patterns of sediments of $L 2(533 \mathrm{~nm})$ formed in three $200 \mathrm{mM}$ buffers with a) $\mathrm{pH} 5$ b) $\mathrm{pH} 8$ and c) $\mathrm{pH}$ 10.8, and d) Profiles plots of the 2D SAXS patterns of a-c and Fig. 9.3.a-c, showing the pronounced structure factor peaks on top of a form factor contribution for water and $\mathrm{pH} 9$.

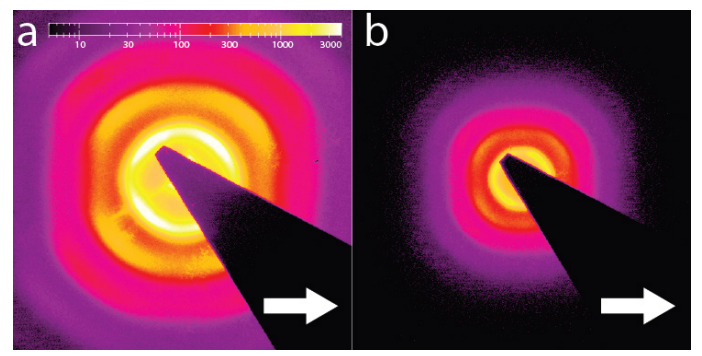

Figure 9.II. 2D SAXS patterns of hematite cubes in 9mM TMAH sedimented in an applied magnetic field of $25 \mathrm{mT}$ of different size (a) L2, D = $533 \mathrm{~nm}$ (B) JI, D = $1055 \mathrm{~nm}$. The white arrow indicates the direction of the magnetic field.
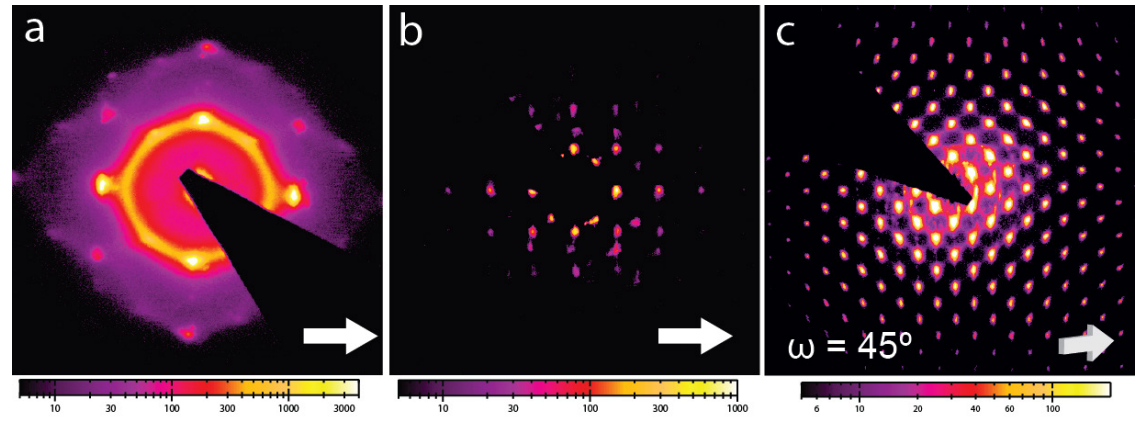

Figure 9.12. 2D SAXS patterns of sediments of silica coated cubes in ethanol in an applied magnetic field of $25 \mathrm{mT}$ (white arrow indicates direction) at $\omega=0^{\circ}$ of a) CI5_Si with $D=340 \mathrm{~nm}$ b) F_Sil with $D=667$ $\mathrm{nm}$, and c) at $\omega=47^{\circ}$ of F2_Si at the sediment top at $z=-0.1 \mathrm{~mm}$ with a long exposure time, showing high order Bragg peaks. 


\section{A.9.5. BCM Structure analysis}

Experimentally we have determined that the cubes are ordered into a BCM structure. The unit cell is described by:

$$
\begin{aligned}
& a=846 \mathrm{~nm} \quad \text { with } \alpha=\beta=90^{\circ} \text { and } \gamma=85^{\circ} . \\
& b=852 \mathrm{~nm} \\
& c=972 \mathrm{~nm}
\end{aligned}
$$

We calculated the lattice parameters for L2_Si with $D=652 \mathrm{~nm}$ and $\mathrm{m}=3.08$ for $\mathrm{C}_{0}$ and $\mathrm{C}_{1}$ according to Jiao et al. ${ }^{[25]}$. Three different orientations of the BCM cell overlapping with the FCC-like unit cell can be realized. The resulting lattice parameters for the $\mathrm{BCM}$ lattice of $\mathrm{C}_{0}$ and $\mathrm{C}_{1}$ are given in Table 9.2.

For the BCM cell described by Marechall et al. ${ }^{[28]}$ for parallel rounded cubes the lattice parameters had to be adjusted to the correct rotation of the unit cell we used for our BCM lattice. The calculated values are shown in Table 9.2 as well.

It is clear from all values that the $c$ value seems to match the best. The values for $a$ and $b$, however, are very far off, by at least $100 \mathrm{~nm}$. Clearly, these simulated structures do not completely describe our crystal lattice. Instead of purely excluded volume interaction, other interactions play a role between our cubes, such as surface charge and magnetic dipole attractions. The high

\begin{tabular}{|c|c|c|c|c|c|c|}
\hline \multirow[b]{2}{*}{ Realization } & \multirow[b]{2}{*}{$\begin{array}{c}\text { Lattice } \\
\text { parameter }\end{array}$} & \multicolumn{2}{|c|}{$\begin{array}{l}C_{0} \text { - lattice } \\
\text { (Jiao et al.) }\end{array}$} & \multicolumn{2}{|c|}{$\begin{array}{l}C_{1} \text { - lattice } \\
\text { (Jiao et al.) }\end{array}$} & \multirow{2}{*}{$\begin{array}{c}\text { BCM } \\
\text { (Mareschal et al.) } \\
\text { Length } \\
(\mathbf{n m})\end{array}$} \\
\hline & & $\begin{array}{l}\text { Length } \\
\text { (D) }\end{array}$ & $\begin{array}{c}\text { Length } \\
(\mathrm{nm})\end{array}$ & $\begin{array}{l}\text { Length } \\
\text { (D) }\end{array}$ & $\begin{array}{c}\text { Length } \\
(\mathrm{nm})\end{array}$ & \\
\hline \multirow[t]{3}{*}{ I } & a & 1.1 & 710.2 & 1.10 & 731.8 & 652 \\
\hline & b & 1.1 & 710.2 & 1.05 & 674.4 & 718 \\
\hline & c & 1.5 & 980.5 & 1.55 & 995.2 & 1041 \\
\hline \multirow[t]{3}{*}{ II } & $\mathbf{a}$ & 1.1 & 710.2 & 1.10 & 731.8 & - \\
\hline & b & 1.1 & 710.2 & 1.05 & 674.4 & - \\
\hline & c & 1.5 & 980.5 & 1.55 & 995.2 & - \\
\hline \multirow[t]{3}{*}{ III } & a & 1.1 & 732.3 & 1.05 & 674.4 & - \\
\hline & b & 1.1 & 732.3 & 1.05 & 674.4 & - \\
\hline & c & 1.6 & 1027.7 & 1.55 & 995.2 & - \\
\hline
\end{tabular}
surface charge will also effectively round off the cubes, and thus lower $m$, we did not take this into account for these calculations.

Table 9.2. BCM lattice parameters for different simulation lattices. 


\section{References}

1. A. Yethiraj and A. van Blaaderen, Nature, 2003, 421, 513-517.

2. V. Malik, A. V. Petukhov, L. He, Y. Yin and M. Schmidt, Langmuir, 2012, 28, 14777-14783.

3. Q. Zhang, M. Janner, L. He, M. Wang, Y. Hu, Y. Lu and Y. Yin, Nano Lett., 2013, 13, 1770-1775.

4. A. van Blaaderen, Nature, 2006, 439, 545-546.

5. S. C. Glotzer and M. J. Solomon, Nature Mater., 2007, 6, 557-562.

6. S. Yang, S. Kim, J. Lim and G. Yi, J. Mater. Chem., 2008, 18, 2177-2190.

7. P. F. Damasceno, M. Engel and S. C. Glotzer, Science, 2012, 337, 453-457.

8. M. OzakiI, H. Suzuki, K. Takahashi and E. Matijevic, J. Colloid Interface Sci., 1986, 113, 76-80.

9. S. H. Lee and C. M. Liddell, Small, 2009, 5, 1957-1962.

10. N. K. Chaudhari, H. C. Kim, C. S. Kim, J. Park and J. Yu, Crystengcomm, 2012, 14, 2024-2031.

11. L. Rossi, Colloidal Superballs, Ph.D. Thesis, Utrecht University, 2012.

12. L. Cromieres, V. Moulin, B. Fourest and E. Giffaut, Colloids Surf. A., 2002, 202, 101-115.

13. A. Snigirev, V. Kohn, I. Snigireva and B. Lengeler, Nature, 1996, 384, 49-51.

14. V. Kohn, I. Snigireva and A. Snigirev, Opt. Commun., 2003, 216, 247-260.

15. M. Drakopoulos, A. Snigirev, I. Snigireva and J. Schilling, Appl. Phys. Lett., 2005, 86, 014102.

16. E. Homan, M. Konijnenburg, C. Ferrero, R. Ghosh, I. Dolbnya and W. Bras, J. Appl. Cryst., 2001, 34, 519-522.

17. A. Petukhov, J. Thijssen, D. t Hart, A. Imhof, A. van Blaaderen, I. Dolbnya, A. Snigirev, A. Moussaid and I. Snigireva, J. Appl. Cryst., 2006, 39, 137-144.

18. J. H. J. Thijssen, A. V. Petukhov, D. C. 't Hart, A. Imhof, C. H. M. van der Werf, R. E. I. Schropp and A. van Blaaderen, Adv. Mater., 2006, 18, 1662-1666.

19. A. Bosak, I. Snigireva, K. S. Napolskii and A. Snigirev, Adv. Mater., 2010, 22, 3256-3259.

20. D. V. Byelov, J. M. Meijer, I. Snigireva, A. Snigirev, L. Rossi, E. van den Pol, A. Kuijk, A. Philipse, A. Imhof, A. van Blaaderen, G. J. Vroege and A. V. Petukhov, RCS Advances, 2013, 3, 15670-15677.

21. S. Sacanna, L. Rossi and A. P. Philipse, Langmuir, 2007, 23, 9974-9982.

22. A. L. Andrade, J. D. Fabris, J. D. Ardisson, M. A. Valente and J. M. F. Ferreira, J. Nanomat., 2012, 454759.

23. A. I. Abrikosov, S. Sacanna, A. P. Philipse and P. Linse, Soft Matter, 2013, 9, 8904-8913.

24. G. B. Alexander, W. M. Heston and R. K. Iler, J. Phys. Chem, 1954, 6, 453-455.

25. Y. Jiao, F. H. Stillinger and S. Torquato, Phys. Rev. E, 2009, 79, 041309.

26. R. D. Batten, F. H. Stillinger and S. Torquato, Phys. Rev. E, 2010, 81, 061105.

27. R. Ni, A. P. Gantapara, J. de Graaf, R. van Roij and M. Dijkstra, Soft Matter, 2012, 8, 12135-12135.

28. M. Marechal, U. Zimmermann and H. Loewen, J. Chem. Phys., 2012, 136, 144506.

29. M. Aoshima, M. Ozaki and A. Satoh, J. Phys. Chem. C, 2012, 116, 17862-17871.

30. G. S. Park, D. Shindo, Y. Waseda and T. Sugimoto, J. Colloid Interface Sci., 1996, 177, 198-207.

31. J.M. Meijer, D. V. Byelov, L. Rossi, A. Snigirev, I. Snigireva, A. P. Philipse and A. V. Petukhov, Soft Matter, 2013, 9 , 10729-10738. 


\section{SUMMARY}

Colloids are small particles $(1-1000 \mathrm{~nm})$ that display Brownian motion in suspension and as a result colloidal suspensions obey the same statistical physical laws as atomic and molecular fluids. Consequen colloidal suspensions show similar phase transitions, for example a fluid to crystal transition. Because of their size, colloids can be easily observed with optical microscopy. Moreover the typical time scale on which processes occur in colloidal systems is much longer than that in atomic systems which facilitates observation of relevant physical processes. As a result colloids are widely used as model systems to study generic processes which also occur in atomic and molecular systems but are much more difficult (if not impossible) to observe in these systems. In addition, due to their specific length scales, structures spontaneously formed by colloids have also gained interest as functional materials.

This thesis discusses the colloidal crystals formed by colloids with two different shapes, namely spheres and cubes. The investigation of colloidal crystal structures spontaneously formed by these particles in suspension, or induced by controlled solvent evaporation, forms the main focus of this thesis. Moreover, we explore several new X-ray imaging and scattering techniques that provide additional information on the colloidal crystal structures. An overview of the two systems and their (predicted) ordered structures, as well as the fabrication and characterization techniques is presented in Chapter 1.

Part A of this thesis deals with colloidal sphere crystals in two forms: dried colloidal crystals of fixed spheres, which are of particular interest as templates for photonic structures, and colloidal crystals in suspension in which colloidal dynamics can be studied. Chapter 2 describes the preparation of dried colloidal crystals via sedimentation and convective assembly; with the aim to study the average structure and defects with synchrotron and free electron laser $\mathrm{X}$-ray techniques. The special sample modifications needed for these experiments during or after preparation are presented. It is shown that for small colloidal crystal grains that need to be mounted on a fiber tip, dried crystals of silica spheres obtained via sedimentation are preferred. Furthermore, polystyrene particles are found to be deposited controllably in thin large single crystals on the specific substrates needed for free electron laser (FEL) studies. In addition the results are discussed of two X-ray experiments performed on polystyrene crystals. The first is a pump-probe experiment of the dynamics of laser heating on the crystal structure with a FEL; the second study addresses the colloidal crystal structure melting around the glass transition temperature of polystyrene.

The colloidal crystal grains mounted on a fiber tip prepared in Chapter 2 are studied with coherent X-ray diffraction imaging (CXDI) in Chapter 3 to reveal the inner structure of the grains. In the scattering data diffuse scattering in the form of a Bragg rod is observed, which is related to stacking defects in the sequence of the hexagonal close-packed planes. A model for the Bragg rod intensity distribution is developed that allows us to extract the stacking sequence 
of the crystal grain. Additionally, preliminary results from the reconstructions of the full real space structure are presented. Both analysis methods open up ways to obtain crucial structural information from finite-sized crystalline samples by employing advanced third generation $\mathrm{X}$-ray sources.

Colloidal crystals in suspension are studied in Chapter 4 where we aim to induce defect diffusion while following the process in real space and time. A new model system is developed in which thermosensitive colloidal poly-N-isopropyl acrylamide (PNIPAM) particles are embedded into a non-thermo-responsive colloidal crystal. Laser scanning confocal microscopy (LSCM) of the crystal structures shows that a decrease in temperature can induce in situ defect movement.

Part B of this thesis deals with the colloidal crystal structures formed by micron-sized colloidal cubes with rounded edges. Their shape is described by a so-called superball that is mathematically defined as: , where $a$ is the particle radius and $m$ is the shape parameter that defines the roundness of the cube corners. The aim in this part of the thesis is to investigate the effect of the cube shape, the role of the $m$-value and the various particle interactions, ranging from repulsive to attractive, on the close-packed structures that these cubes spontaneously form.

Chapter 5 describes the synthesis of three different types of colloidal cubes: hematite cubes, silica coated hematite cubes and hollow silica cubes. The properties of the three different cubes such as size, shape and interaction are briefly outlined as these play an important role in part B of the thesis.

Chapter 6 and Chapter 7 focus on the ordered structures formed by hollow silica cubes induced via convective assembly, which is a convenient method for the fabrication of ordered structures of colloids. In Chapter 6 an experimental setup is introduced that employs a thermal gradient to counteract sedimentation of the particles, which allows the fabrication of ordered structures of the cubes. Scanning electron microscopy (SEM) analysis of the structures shows that due to the strong capillary forces cube sides are aligned with the substrate. This alignment explains that the order found in monolayers corresponds to the two optimal packings, the $\Lambda_{0}$ and the $\Lambda_{1}$-lattice, as predicted by theory and simulations for 2D superball structures. Small angle X-ray scattering measurements on 3D multilayer structures confirm the presence of the lattices in the layers, which appear to possess a hollow-site stacking. Chapter 7 focusses on cubes with a higher $m$-value that is a consequence of a larger cube size. Three different convective assembly methods are applied to counteract cube sedimentation. One of these methods has the advantage that the formation of ordered structures can be followed in time with optical microscopy. The analysis reveals that during structure formation rearrangements can take place in the close-packed structures. Computer analysis of the SEM images is used to identify the local packing environment of each particle. This analysis leads to conclusive evidence that an increase in $m$ corresponds to an increase in the $\Lambda_{1}$-lattice packing in monolayers, as also predicted by simulations. 
Motivated by the insight that repulsive particles may display phase transitions driven by entropy only, we investigate in Chapter $\mathbf{8}$ the assembly of charge-stabilized hollow silica cubes in suspension. It is shown that Debye screening length influences the self-assembly of the cubes in aqueous suspensions and a critical Debye length $(\sim 48 \mathrm{~nm})$ is identified above which crystallization can occur. The dense crystalline sediments that are obtained under gravity are investigated in detailed with SAXS. These measurements show that although the cubes are aligned to the capillary walls, the formed structures still seems to be in agreement with the equilibrium structures predicted by simulations of hard cubes.

In Chapter 9 the focus is shifted to the hematite cubes with a permanent magnetic dipole moment to study the effect of the dipole-dipole attractions on the cube assembly into sedimentary crystals. Detailed SAXS investigations reveal that by increasing the double-layer repulsion, which opposes the magnetic dipole-dipole attractions and Van der Waals attractions, ordering can be induced in the cube sediments. In different solvent and/or salt conditions the structures can be tuned from short-range ordered polycrystalline sediments to long-range ordered layered structures of cubes. In addition, the assembly can be directed by the presence of an external magnetic field, which is found to induce the formation of a single body centred monoclinic (BCM) crystal structure that possesses true long-range order. Surprisingly, a rotation of the $\mathrm{BCM}$ structure is found to occur in the lower parts of the sediments as a consequence of the increased osmotic pressure, which destroys the alignment of the cubes in the magnetic field. 


\section{NederLandse SamenVatTing}

\section{VOOR IEDEREEN}

Een van de fascinerendste natuurkundige fenomenen is de spontane overgang van een vloeistof naar een vaste stof, zoals het bevriezen van water of het kristalliseren van zout uit een oplossing. Helaas is het niet mogelijk om dit op het niveau van de kleinste bouwstenen, dat van atomen en moleculen, gedetailleerd te bestuderen. Om dit toch te proberen, gebruiken onderzoekers vaak modellen. Eén van deze modellen zijn colloïdale suspensies. Dit is zijn kleine deeltjes met afmetingen tussen 1 nanometer en 1 micrometer ( 1 duizendste millimeter!) die in een vloeistof zitten. Figuur 11.1 laat een glazen buisje zien dat dezelfde dikte heeft als een menselijke haar (2 honderste millimeter) waarop colloïden (de kleine bolletjes) zitten. De bolletjes zijn vele malen kleiner dan deze buis en dat verklaart waarom je colloïden met het blote oog niet kunt zien. Verassend genoeg zijn veel vloeistoffen in het dagelijks leven colloïdale suspensies, zoals melk, bloed, verf en dagcrème.

Doordat colloïden zo klein zijn vertonen ze in een vloeistof spontane thermische beweging (Brownse beweging), als gevolg van deze thermische bewegingen vertonen ze hetzelfde fasegedrag als atomen en moleculen. De Brownse beweging kun je voorstellen als een danseres die al dansend door een hele danszaal heen beweegt; dan weer links, dan weer rechts, dan een draai, zonder dat ze echt een doel lijkt te hebben. De fases,

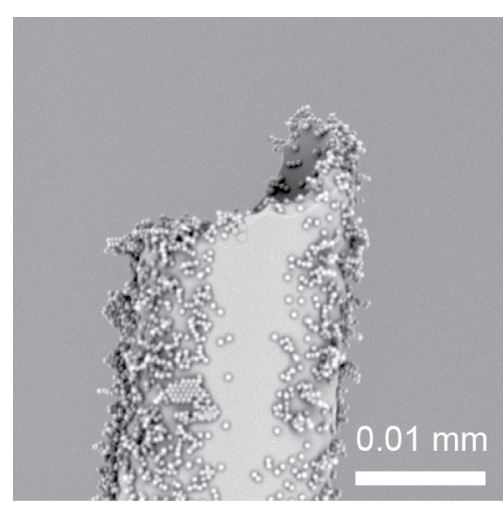

Figuur II.I. Glazen buisje met de dikte van een menselijke haar waar colloïden op zitten. ofwel toestanden, waar colloïden zich in kunnen bevinden, zijn onder andere een gas, vloeistof en kristal. Of de colloïden deze fase laten zien hangt af van de concentratie colloïden in een vloeistof. De spontane kristallisatie gebeurt alleen als de concentratie hoog genoeg is. Dit proces kun je vergelijken met de bevriezing van water als de temperatuur onder nul graden komt. Door de grootte van de collö̈den kunnen we met een microscoop de kristallisatie op deeltjes niveau bestuderen en kunnen we iets zeggen over de drijvende krachten achter het proces.

In een kristal zitten alle deeltjes netjes in een rooster. Figuur 11.2. toont een kristal gevormd van de colloïden die we hiervoor op een glazen buisje zagen. Het lijkt bijna alsof iemand de bolletjes netjes naast elkaar gelegd heeft maar dit is spontaan gebeurd. De doorsnede laat zien dat de bolletjes een hexagonaal rooster hebben gevormd. Waarom de colloïden spontaan op een rooster gaan zitten, ligt aan de vrije ruimte die elk deeltje zo behoudt om rond te kunnen bewegen. We kunnen dit begrijpen als we de danseres er weer even bijhalen. Stel, we stoppen haar samen met een heleboel andere dansers in een kleine ruimte. Als zij en al die andere mensen toch nog graag willen dansen, moeten ze een oplossing bedenken, want zouden ze 


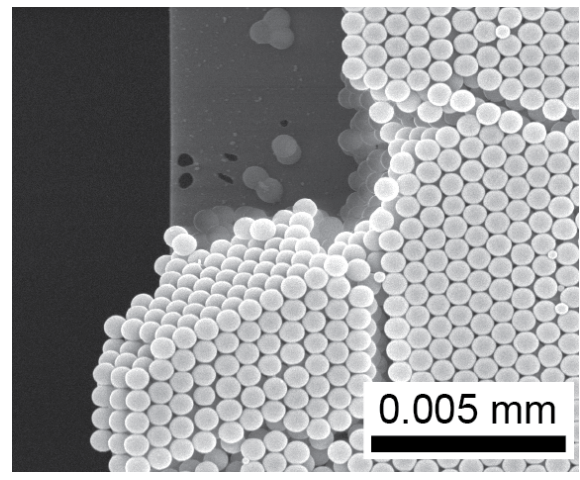

Figuur II.2. Kristal van bolvormige colloïden. De bolletjes hebben spontaan deze structuur gevormd.

wild blijven rondlopen dan komt iedereen vast te zitten. Het beste is als iedereen op een vast plekje gaat staan zodat ze nog een beetje kunnen bewegen, zoals bij concerten ook vaak gebeurt (vooraan bij het podium geldt dat natuurlijk niet meer).

Het bestuderen van de colloïdale kristallen geeft veel inzicht in de eigenschappen van kristallen van atomen en moleculen. Zo kunnen we bestuderen welke rooster deeltjes met verschillende vormen maken maar ook wat voor foutjes er in dat kristal zitten. De vorm is erg belangrijk want met bollen kun je heel andere bouwwerken maken dan met kubussen, zoals een kleuter in de bouw hoek al leert. De foutjes in een kristal worden kristaldefecten genoemd en bestaan bijvoorbeeld uit het ontbreken van een deeltje op een plek of juist een deeltje te veel. Daarnaast kunnen ook ingewikkeldere fouten voorkomen, zoals twee roosters die niet helemaal overeen komen of roosters die gedraaid zijn ten opzichte van elkaar. In kristallen van atomen, zoals metalen of zoutkorrels, komen zulke fouten ook voor en die kunnen de eigenschappen beïnvloeden. Metalen met te veel fouten in hun kristalrooster kunnen heel zwak worden en snel breken. Het is dus belangrijk te begrijpen welke invloed kristaldefecten hebben.

Colloïden kunnen tegenwoordig van verschillende materialen maar ook met verschillende vormen gemaakt worden. Hierdoor kunnen onderzoekers ze steeds beter gebruiken als vergelijking voor de kristallen van atomen en moleculen en op deze manier steeds meer ingewikkelde processen uit de natuur nabootsen. Daarnaast zijn de structuren die de colloïden kunnen vormen ook interessant voor verschillende toepassingen. Ze hebben namelijk dezelfde grootte als de golflengte van het zichtbare licht en verstrooien het licht daardoor. Een voorbeeld hiervan zijn de opalen, die hun mooie kleuren danken aan hun kern van silica bollen van ongeveer 500 nanometer. Met colloïden kunnen we dus nieuwe materialen met bijzondere eigenschappen maken.

In dit proefschrift hebben we gekeken naar de invloed van de vorm van de colloïden op het kristalrooster. Hiervoor hebben we twee verschillende vormen gebruikt; bollen, die worden besproken in Deel A en kubussen gepresenteerd in Deel B. We hebben voornamelijk op twee verschillende manieren kristallen van deze twee vormen gemaakt. De eerste manier is de concentratie van de colloïden in de vloeistof verhogen zoals boven beschreven voor de dansers. Dit hebben we gedaan door ze te laten uitzakken (sedimenteren) in de vloeistof, waarbij de zwaartekracht een handje helpt. De tweede manier is door de vloeistof gecontroleerd te laten verdampen. De laatste techniek wordt ook wel het koffievlekeffect genoemd en heeft iedereen wel eens op tafel waargenomen. Door de verdamping van vloeistof waar deeltjes in zitten, verzamelen door de vloeistofstroming alle deeltjes aan de rand van de druppel. Dit is de reden dat een koffievlek vaak donkerder is aan de randen. In Hoofdstuk 1 worden deze technieken en 
de manier waarop we de kristallen kunnen bestuderen beschreven.

Kristallen van colloïdale bollen zijn al gedetailleerd bestudeerd daarom is het doel in Deel A om de kristaldefecten met nieuwe experimentele technieken en op nieuwe manieren te bestuderen. In Hoofdstuk 2 wordt daarom eerst beschreven hoe je kristallen van bollen op de twee manier, sedimentatie en vloeistofverdamping, kunt maken. Met verschillende soorten microscopie (met optisch licht of elektronen) is er bekeken of de structuren juist waren. Hierna zijn de kristallen aangepast zodat ze voldoen aan de eisen van de nieuwe experimentele technieken. Figuur 11.3 laat twee voorbeelden zien van een kleine kristallen van bollen die op het uiteinde van vezels geplakt zijn. We hebben bepaald van welk materiaal de colloïden het beste gemaakt konden worden, plastic of glas, en welke kristallisatiemanier hiervoor het beste was.

In Hoofdstuk 3 bestuderen we een van deze kristallen op een vezel met een nieuwe techniek, die gebruikt maakt van verstrooiing van röntgenstraling. Röntgenstraling wordt ook in het ziekenhuis gebruikt voor het bestuderen van je botten, maar in deze techniek gebeurt het op een hele kleine schaal. Hierdoor konden we de binnenkant van het kristal bestuderen en hebben we ontdekt dat er een specifiek soort kristaldefect, namelijk een stapelingsfout, in de kristalkorrel aanwezig was.

In Hoofdstuk 4 bestuderen we de kristaldefecten op een andere manier. Hier maken we gebruik van colloïden die op sponzen lijken. Bij lage temperatuur zitten ze vol water maar bij hogere temperatuur worden ze helemaal leeg geknepen. Hierdoor verandert hun grootte en dus de concentratie. Welke invloed dit op de kristal structuur heeft, hebben we bestudeerd met confocale microscopie. Dit is een speciale microscoop waarbij een laser en een diafragma gebruikt worden waardoor we de driedimensionale structuur van het colloïdale kristal in de vloeistof kunnen bekijken. Deze studies toonden aan dat als de colloïden opzwellen (dus als we van hoge naar lage temperatuur gaan) er fouten in de kristallen van plek veranderen en deeltjes gaan bewegen. Dit brengt ons weer iets dichterbij de vergelijking met atomen en moleculen want in hun kristallen zijn bewegingen van de kristaldefecten al waargenomen.

In Deel B van dit proefschrift hebben we gekeken naar de kristallen gevormd door colloïdale
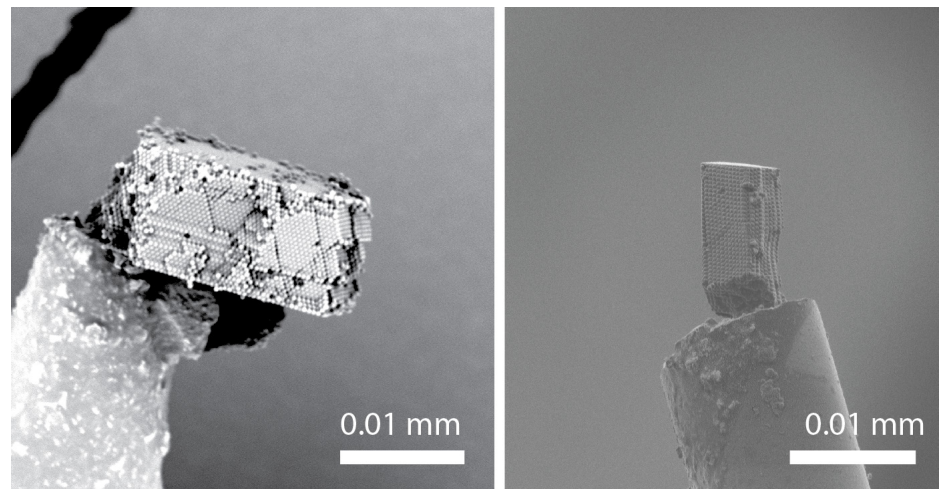

Figuur II.3. Voorbeelden van kleine kristallen van colloïden op de punt van een vezel. 
kubussen. Allereerst bespreken we in Hoofdstuk 5 hoe deze kubusvormige colloïden gemaakt worden en welke eigenschappen ze hebben. We kunnen drie varianten maken; hematiet (ijzeroxide) kubussen, hematiet kubussen met een glas (silica) laagje om de buitenkant en holle silica kubussen.

In Hoofdstuk 6 en Hoofdstuk 7 focussen we op de holle silica kubussen en kijken we welke kristallen ze kunnen maken als we de vloeistof verdampen (koffievlekeffect). In Hoofdstuk 6 hebben we uitgezocht welke condities (temperatuur, vloeistof etc.) we hier het beste voor kunnen gebruiken. Het blijkt dat als we water gebruiken we mooie geordende structuren kunnen maken. De structuur hebben we onderzocht en laat zien dat er twee verschillende manieren zijn waarop deze kubussen, die afgeronde hoekjes hebben, gestapeld kunnen worden. Daarna hebben we in Hoofdstuk 7 gekeken wat er gebeurd als je de kubus hoekjes minder afgerond maakt. Er was door computer simulaties voorspeld dat in dit geval de kubusjes dan maar op een van de twee eerder genoemde manieren zouden stapelen, namelijk met hun afgeronde hoekjes tegen elkaar aan. Ons onderzoek laat zien dat dit inderdaad gebeurt. Figuur 11.4. laat een foto zien de kubussen die op deze manier gestapeld zijn.

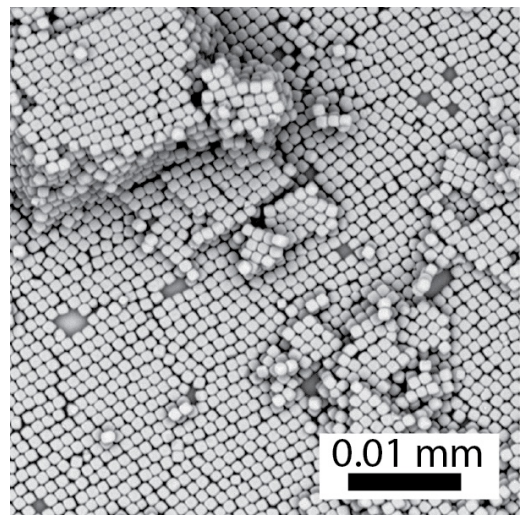

Figuur II.4. Structuur gevormd van colloïdale kubussen door vloeistofverdamping.

Omdat we weten dat ook door sedimentatie kristallen gevormd kunnen worden, onderzoek we in Hoofdstuk 8 wat voor kristallen de holle silica kubussen in een vloeistof vormen. We hebben dit met behulp van kleine hoek röntgenverstrooiing gedaan. In het Engels heet dit small angle X-ray scattering en wordt afgekort als SAXS. Op deze manier hebben we informatie verkregen over de kristal structuur. Het blijkt uit onze gedetailleerde analyse dat de kubussen zo spontaan geordende structuren vormen. Het leuke is dat deze structuren ook al door mensen voorspeld waren met computer experimenten en wij hebben die kunnen bevestigen.

In Hoofdstuk 9 bestuderen we de hematiet kubussen die magnetisch zijn en elkaar daardoor aantrekken. We hebben gekeken naar wat de invloed van deze aantrekking tussen de kubussen is op de structuren die ontstaan tijdens het sedimenteren. De soort vloeistof waar de kristallen inzitten blijkt van belang te zijn. Ook het hebben van een silica laag blijkt van invloed te zijn op de aantrekkingen tussen de kubussen en zorgt dat er structuren die langeafstandsorde bevatten ontstaan. Daarnaast hebben we de kubussen laten sedimenteren in een magneetveld waardoor alle kubussen dezelfde richting op gaan staan en een bijzonder kristal vormen.

\section{Dankwoord}

Tim, Tiemen, Marleen en Karin worden bedankt voor het beoordelen van de leesbaarheid en voor het corigeren van deze samenvatting. 


\section{ACKNOWLEDGEMENTS}

\section{DANKWOORD}

De afgelopen jaren hebben een heleboel mensen in allerlei verschillende vormen een bijdrage geleverd aan de totstandkoming van dit proefschrift. Hier wil ik iedereen graag bedanken en ik hoop dat ik niemand vergeet.

Allereerst, Andrei, dank je, thank you, Спасибо! Jij was de reden dat ik toch al na een paar maanden weer in het lab stond in plaats van mijn geplande jaar koffie serveren. Bedankt voor je oneindige enthousiasme, het feit dat je deur altijd open staat (zelfs als er grote ESRF stapels lagen) en voor de vele 'gele hoeden'. Ik heb de afgelopen jaren een heleboel van je geleerd, al dan niet tijdens de nachtdiensten bij de ESRF, over verstrooiing maar ook Russisch en de andere belangrijke dingen in het leven. Daarnaast waardeer ik de grote vrijheid die je me hebt gegeven om in de vele projecten mijn eigen plan te trekken. Ook wil ik hier van de gelegenheid gebruik maken om Anna te bedanken voor de interesse en steun tijdens de afgelopen jaren. Ik zal het dansen tijdens de SAS in Sydney niet snel vergeten.

Uiteraard wil ik ook Henk bedanken voor de mogelijkheid om als allerlaatste student (misschien nu toch echt?) mijn promotieonderzoek onder zijn begeleiding te doen. Ook al vond je begeleiding grotendeels van een afstand plaats, je wist me altijd weer op het juiste spoor te zetten en focus aan te brengen in mijn onderzoek. Bovenal wil ik je bedanken voor je hulp en enthousiasme tijdens de afronding van mijn proefschrift. Jouw oog voor planning gecombineerd met jouw oneindige kennis van de literatuur heeft mijn proefschrift gemaakt tot wat het nu is.

Ook wil ik Albert bedanken voor zijn vele bijdragen aan het tweede deel van mijn proefschrift. Ondanks dat je pas officieel een half jaar geleden mijn tweede promotor bent geworden, was je altijd erg geïnteresseerd in mijn onderzoek. Bedankt voor de vele wetenschappelijke discussies en de kritische en gedetailleerde manier waarop je mijn gehele proefschrift hebt doorgelezen.

A large part of this thesis is based on synchrotron data which has been obtained at different facilities in collaboration with many people. First of all I would like to thank all the staff and especially, Giuseppe, Daniel, Dirk and Florian from the DUBBLE beamline at the ESRF in France. Without your expert technical and measurements skills it would have been impossible to measure my 'huge' cubes. Thank you for all the help, even during the night and weekends, and the optimistic approach we could always count on. I would also like to thank Anatoly and Irina of ID6 for allowing us to measure on the X-ray microscope, as well as for their contributions to the different subjects.

Another big part of my $\mathrm{PhD}$ has been spent working in collaboration with or actually in Hamburg, Germany with the group of Ivan Vartanyants at Hasylab. Ivan, I would like to thank 
you for your enthusiasm during each experiment and critical view on the results. Johannes, I want to thank you for my warm welcome in Hamburg during the first FLASH experiments at DESY and making the whole experience an enjoyable chaos. Anatoly, thanks for the nice collaborations we had on our projects trying to understand each other's calculations. I want to thank Ruslan, Dima, Oleg and Oleksandr for keeping the night-shifts at FLASH and LCLS full of life and for improving my Russian. I also want to thank the staff of the P10 beamline and especially Alexey for the nice collaborations on the colloidal crystal films.

During my $\mathrm{PhD}$, I also had the opportunity to visit for three months the applied physics lab at RMIT in Melbourne, Australia. I want to thank Gary for giving me this opportunity and welcoming me back into the lab. I also want to thank all the people who made my stay very enjoyable. Andre, Arwen, Billy, Des, Matthew, Reece, Sal and Stephanie: thank you for all the fun and coffee breaks. Also, I owe a special thanks to Phil and Peter for helping me around the lab.

Ook wil ik graag al mijn collega's waar ik de afgelopen jaren mee heb samengewerkt bij het Van 't Hoff lab bedanken, waaronder een aantal in het bijzonder. Jan, ik ben jou veel dank verschuldigd voor je hulp bij het opstarten van mijn eerste projecten tot het doen van metingen bij de ESRF. Bedankt dat je altijd mijn vele vragen van zeer geduldige en duidelijke uitleg voorzag en meer dan eens richting gaf aan mijn onderzoek. Dima, thank you for all the nights spend doing SAXS measurements and watching (scary) movies. Your life lessons and just-do-it attitude have continued to contribute to my work as a scientist. Laura, since our first experiments on the cubes they have become a large part of my thesis. I want to thank you for all our fruitful collaborations but also for all discussions on science and life with and without chocolate cake and cocktails. Mikal, bedankt voor alle stipte koffiepauzes en kritische blik op onderzoek maar ook de rest van de wereld. Ik wil hier ook graag Merel maar ook Tijn en Luc bedanken voor alle dinertjes, spelletjesavonden met zombie achtervolgingen en vakanties. Anke en Susanne, bedankt voor lol en gezelligheid maar ook voor alle keren dat jullie mijn gezeur aanhoorden sinds we ons masteronderzoek bij CMI deden. Anke, we hebben alle ups en downs van het onderzoek kunnen delen, vooral als we samen metingen deden bij de ESRF en er niets zichtbaar was of de beam weer wegviel. Bedankt voor je enthousiasme en het feit dat je bijna altijd zegt wat er in je opkomt. Susanne, bedankt dat je altijd precies weet welke wijsheid ik nodig heb om mijn onderzoek maar ook alle andere zaken weer in perspectief te kunnen zetten. Jouw steun en vrolijkheid hebben de maanden dat ik aan het schrijven was een stuk dragelijker gemaakt en ik ben dan ook heel erg blij dat je mijn paranimf wil zijn.

I would also like to thank all my other colleagues I have had the pleasure to work with during my PhD. First of all I want to thank my office mates: Jan, Daniela, Raja, Jaakko, Esther G., Bas and Antara, thank you for the many discussions, gezelligheid and endless supply of candy. 
Daniela, bedankt voor al je advies in het eerste jaar van mijn promotie. Bas and Antara, thanks for all the dumbbell trivia and the fun times we had learning new lessons. For all the other fun times, coffee breaks with the no-science-at-the-coffee-table-rule, drinks, new year's dinners, conferences, FOM-physics\&CHAINS dancing nights and all the other crazy stuff one can do for five euros, my thanks goes out to all current and former FCC members: Agnieska, Ben, Bob, Chris, Dzina, Esther v.d. P., Fuqiang, Gert Jan, Jan G., Jasper, Joost, Jos, Julius, Kees, Lia, Mark, Maurice, Nina, Ping, Remco, Roel, Rob, Rocio, Sandra, Samia, Sonja, and Yong. Ook wil ik Bonny, Dominique, Emile, Kanvaly, Marina en Stephan maar ook Chris en Hans van de TEM faciliteit bedanken voor alle chemische, technische en administratieve ondersteuning, zonder jullie had ik niet veel gedaan gekregen. Willem, ook al ben je niet direct bij mijn projecten betrokken geweest, ik was altijd welkom met mijn vele vragen en frustraties. Jouw enthousiasme en denderende lach zijn erg aanstekelijk en je hebt me meer dan eens geholpen waar ik je erg dankbaar voor ben. Also thanks to the colleagues from the SCM group, especially Anjan, Fabian, Frank, Henriëtte, Laura F., Marlous en Nina for the discussions and fun times during many Debye meetings, schools and conferences.

A big thank you goes out to all my students; I have had a lot of fun supervising you! Fabian, je was mijn eerste student en hebt bergen werk verzet. Ik ben nog steeds erg trots op onze publicatie en vind het erg leuk om te zien dat je nu aan je eigen promotieonderzoek bezig bent. Daan, je werkte aan een project dat helaas niet altijd even gemakkelijk was. Heel erg bedankt voor je doorzettingsvermogen maar vooral ook voor je enthousiasme en humor tijdens het werk en de koffiepauzes. Cesare, I want to thank you for your persistence during the follow-up project, even if it sometimes resulted in a 'single' particle synthesis. You were the one that finally made the difficult systems work. Your Italian enthusiasm and interest in the research made supervising you a lot of fun. Samia, jouw onderzoekende houding en zelfstandigheid zijn erg indrukwekkend. Bedankt voor al je harde werk aan de geinverteerde kristallen, die helaas mijn proefschrift niet gehaald hebben. Vera, je bleef me verassen met nieuwe ideeën en had ze vaak al uitgevoerd voor ik er erg in had. Bedankt voor al je harde en mooie werk en ik zal ons bezoekje aan de cleanroom in Twente niet gauw vergeten.

Ik wil ook graag de eerste- en tweedejaarsstudenten die een bijdrage aan mijn onderzoek hebben geleverd bedanken voor hun inzet. Emily, Thijs, Jasper en Jens, jullie project was uitdagend mede door het proberen te overzien van zulke lange mannen door de dames. Lisa en Marc, bedankt voor jullie enthousiasme en succesvolle microscopie experimenten. Kristian, Maarten, Robbert en Joeri, bedankt voor jullie enthousiasme en focus wat tot zeer mooie resultaten heeft geleidt. Maarten-Harm en Harriët, mede dankzij jullie oog voor detail hebben jullie heel mooie resultaten geboekt, die posterprijs hebben jullie dan ook dubbel en dwars verdient. 
Ik wil ook graag mijn vrienden bedanken voor alle steun, plezier en afleiding gedurende de afgelopen jaren.

Jorien, Juul, Marleen, Karin en Tinka (en aanhang) bedankt voor alle gezellige weekendjes weg, vakanties, avondjes op de bank hangen en nog veel meer! Jullie interesse (maar kijk je dan de hele dag naar wiebelende deeltjes?), steun en het altijd maar proberen te antwoorden van al mijn waarom vragen over allerlei verschillende onderwerpen de afgelopen jaren waardeer ik heel erg.

Angelique, sinds onze eerste ontmoeting tijdens de introductie zijn we vrienden. Bedankt dat je er altijd bent als het nodig is en dat je me uiteindelijk op de racefiets hebt gekregen. Ik kijk uit naar nog vele rondjes.

Voor alle andere gezellige avondjes, dinertjes, stedentripjes, primusbezoekjes, concerten, wintersporten en festivals wil ik graag Anoeska, Arjan, Fanny, Joost W., Timo, Bieneke, Frank, Anne-Marie, Peter, Nikki, Joost v.d.L., Els, Robbert, Paul, Christine, Remi, Robin, Hilde, Matti Sander en Piter (en iedereen die ik vergeet) bedanken! Frank, ook nog een speciaal bedankje omdat je een geweldige danspartner bent.

Ik wil hier ook graag alle dames en trainers van Fraternitas bedanken voor de gezelligheid. De compleet andere wereld op de donderdag was altijd een heerlijk moment om alle promotiezorgen weer even te vergeten.

Ten slotte wil ik mijn familie bedanken. Ik wil vooral mijn moeder bedanken dat ze me geleerd heeft dat ik alles zelf kan, me gemotiveerd en gesteund heeft, er altijd is als ik haar nodig heb en me zelfs komt opzoeken aan de andere kant van de wereld. Ook wil ik Arie, Joep, Milda, Liucija, Winnie, Tiemen, en mijn vader, Carla, Sabine en Martijn bedanken voor alle liefde en steun de afgelopen jaren ook al begrepen jullie niet altijd waar ik mee bezig was. Tiemen, ook bedankt voor al het (last-minute) corrigeren van mijn Nederlands.

Hier wil ik ook mijn onofficiële schoonfamilie Martin, Marja en Thijs bedanken voor alle interesse en warmte de afgelopen vier jaar.

Winnie, je bent fantastisch! Ontzettend bedankt dat je er altijd voor me bent, trots op me bent en in me gelooft. Hoewel het natuurlijk niet anders zou kunnen, ben ik heel erg blij dat je mijn paranimf bent.

Tim, er zijn niet genoeg woorden (of taarten, maar ik zal het proberen) om je te bedanken voor alle liefde, steun en begrip dat je me hebt gegeven tijdens de afgelopen jaren en dan vooral de laatste maanden. Met jou durf ik elke uitdaging aan. 


\section{CurRiculum VitAE}

Janne-Mieke Meijer was born on the $21^{\text {st }}$ of April in 1985 in Utrecht, the Netherlands. In 2003 she graduated from the St. Bonifatius College in Utrecht. The same year she started her bachelor studies in Chemistry at Utrecht University. She wrote her bachelor thesis on in-plane stacking faults in hard sphere colloidal crystals in the Physical and Colloid Chemistry group at Utrecht University under the supervision of V. de Villeneuve and Dr. A.V. Petukhov. In 2006, she obtained her Bachelor of Science degree in Chemical Sciences. In that same year she became a board member of the chemistry study association "U.S.S. Proton" and started her master program Chemistry and Physics. She performed her master thesis research under supervision of L. Aarts and Prof. A. Meijerink on downconversion of light using lanthanide ion complexes. In 2009, she spent six months as a visiting scholar in the group of dr. G. Bryant and prof. W. van Megen at RMIT in Melbourne, Australia, where she worked on the crystallization of two different hard sphere systems. After obtaining her Master of Science degree in Chemical Sciences (cum laude) from Utrecht University in 2009, she spent a year working as a researcher in the Physical and Colloid Chemistry group before initiating her $\mathrm{Ph}$. D. research project in the same group. Her Ph.D. project focused on the structural and defect analysis of colloidal crystals consisting of different building blocks shapes, namely spheres or cubes, with real and reciprocal space techniques under supervision of Dr. A.V. Petukhov, Prof. A.P. Philipse and Prof. H.N.W. Lekkerkerker. The results of that research are reported in this thesis. 
